\title{
AN IMPLEMENTATION OF THE FACETED CLASSIFICATION SYSTEM FOR SOFTWARE REUSE
}

\author{
By \\ RAM R. MAREDDY \\ Bachelor of Technology \\ Jawaharlal Nehru Technological University \\ Hyderabad, India \\ 1988 \\ Submitted to the Faculty of the \\ Graduate College of the \\ Oklahoma State University \\ in partial fulfillment of \\ the requirements for \\ the Degree of \\ MASTER OF SCIENCE
}

December 1994 
AN IMPLEMENTATION OF THE FACETED CLASSIFICATION SYSTEM

FOR SOFTWARE REUSE

Thesis Approved:

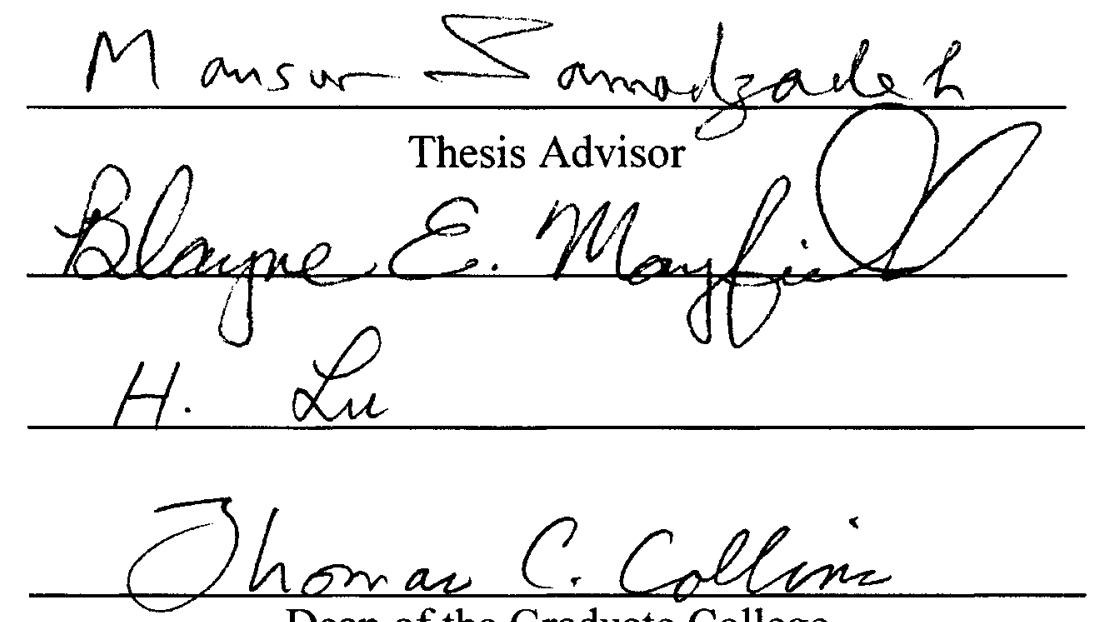

Dean of the Graduate College 


\section{ACKNOWLEDGMENTS}

I take this opportunity to express my gratitude to Dr. Mansur Samazadeh for his guidance and encouragement. Though my thesis work took a lot more time than the usual, Dr. Samadzadeh gave me continuous moral support. I appreciate him greatly for this personal and intellectual relationship. I also like to thank Drs. Mayfield and Lu for agreeing to be on my thesis committee, and for their comments and advice.

I also like to thank my family and friends for their friendship and support. 


\section{TABLE OF CONTENTS}

Chapter

Page

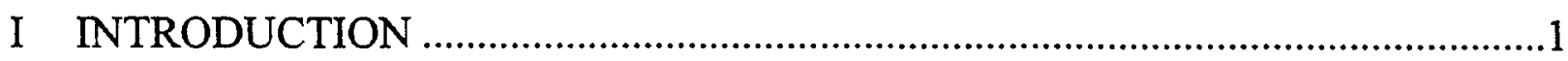

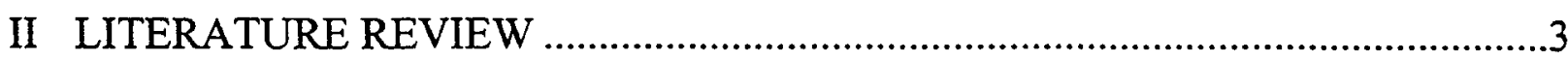

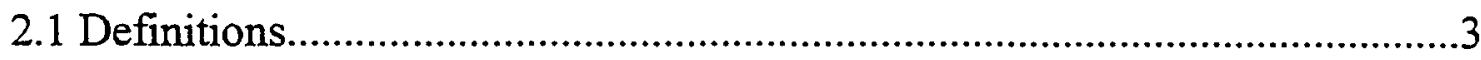

2.2 Software Reuse ..............................................................................

2.2.1 History and Past Research Efforts ....................................................4

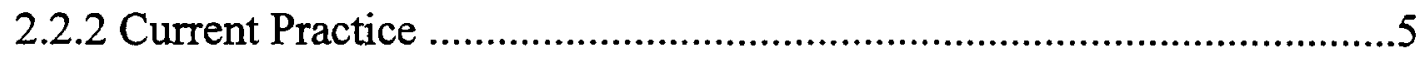

2.2.2.1 Vertical Reuse or High-Level Languages...................................5

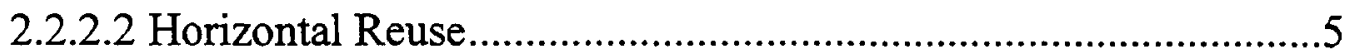

2.2.2.3 Design and Code Scavenging ..............................................6

2.2.2.4 Source Code Components...................................................6

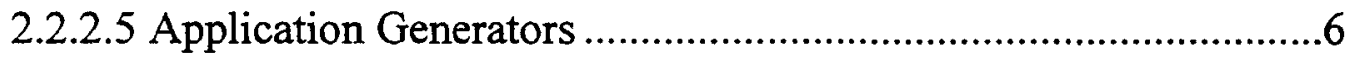

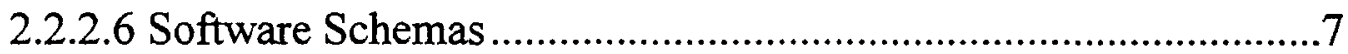

2.2.3 Reuse Classification Criteria...............................................................

2.2.3.1 Abstraction Level ................................................................

2.2.3.2 Customization Methods .........................................................

2.2.3.3 Reusability Conditions............................................................

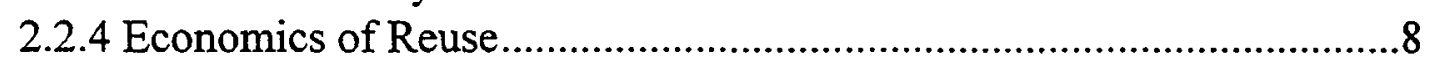

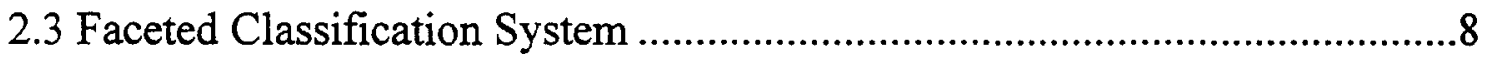

2.3.1 Facets and Vocabulary .................................................................10

2.4 Relational Database Management...........................................................11

2.4.1 Entity-Relationship Diagram for the Tool .....................................13

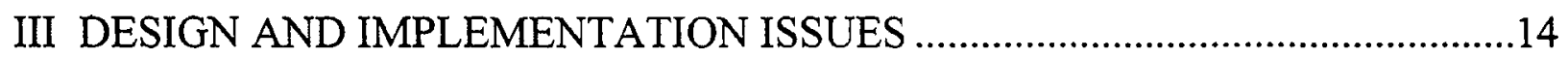

3.1 Implementation Platform and Environment............................................14

3.1.1 Personal Computers Today ........................................................14

3.1.2 DOS Operating System ............................................................15 
3.1.3 Windows Graphical Environment.................................................15

3.2 Specification and Design of the Tool.....................................................16

3.2.1 User Interface .............................................................................16

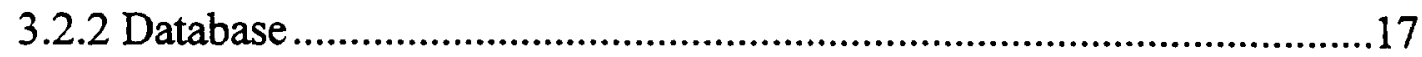

3.3 Development and Implementation of the Tool ..........................................19

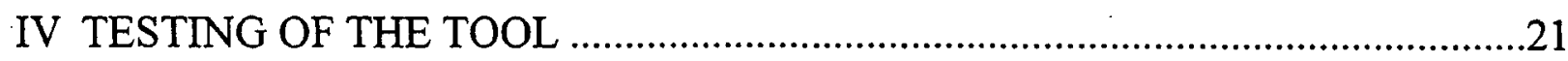

4.1 Sample Reuse Done with the Tool.......................................................21

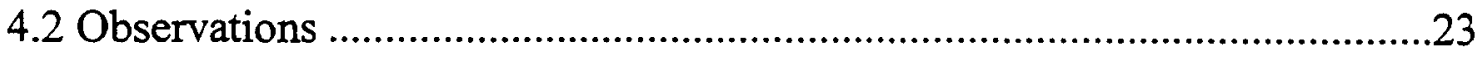

V SUMMARY, CONCLUSIONS, AND FUTURE WORK ....................................24

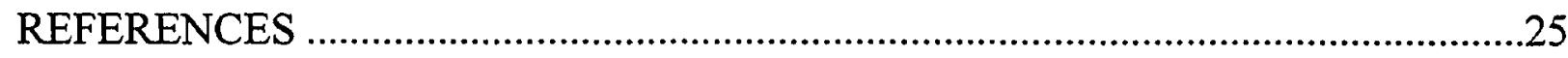

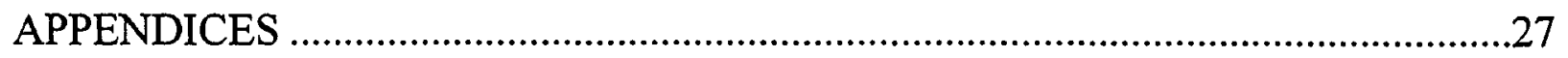

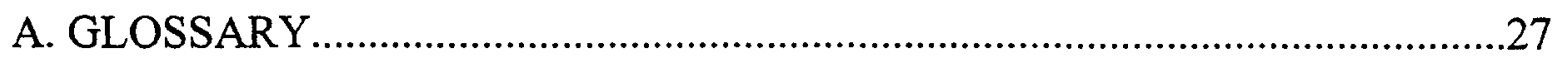

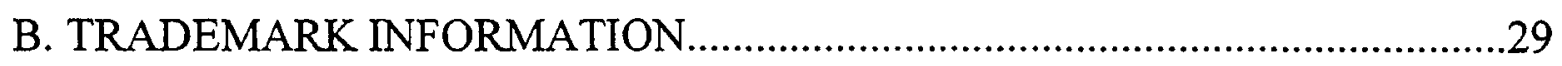

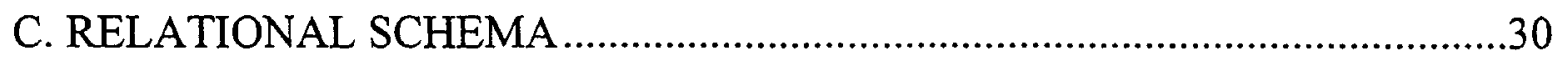

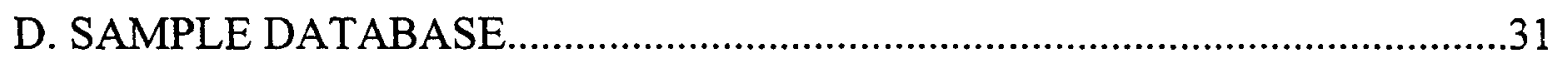

E. USER AND SYSTEM ADMINISTRATOR GUIDE ......................................33

1. Introduction

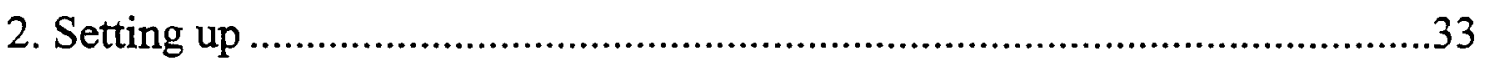

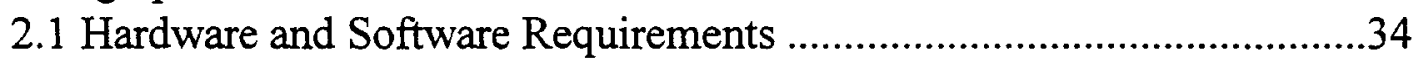

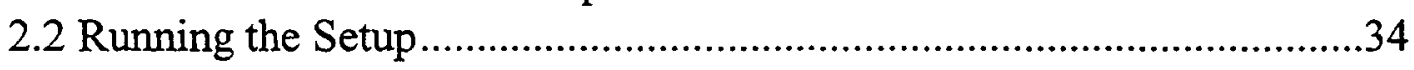

3. Insertion of Software Artifacts into the System...........................................35 


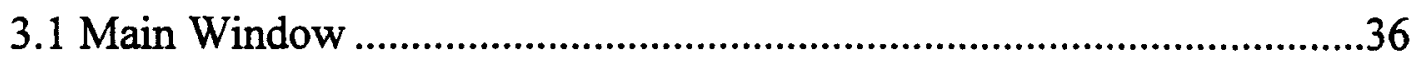

3.2 Preparation of Software Artifacts .........................................................36

3.3 Classification of Software Artifacts.....................................................38

4. Retrieval of Software Artifacts from the Systems .........................................39

4.1 Search of the Repository ....................................................................40

4.2 Synonyms......................................................................................

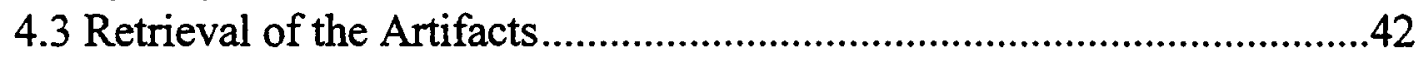

5. System Administration ............................................................................43

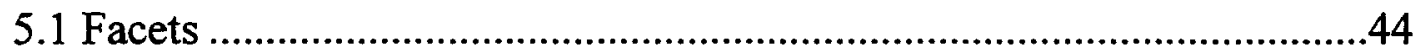

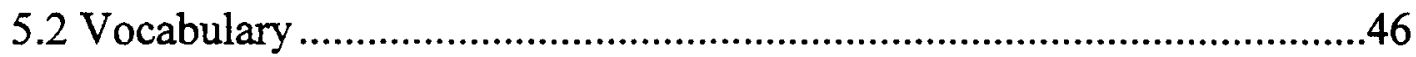

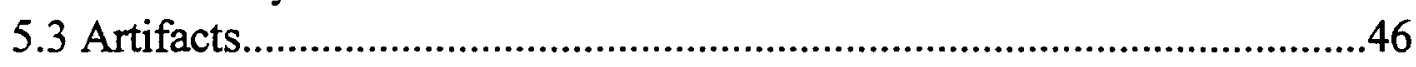

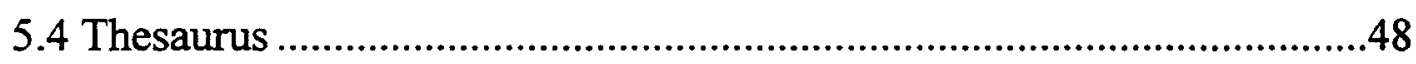

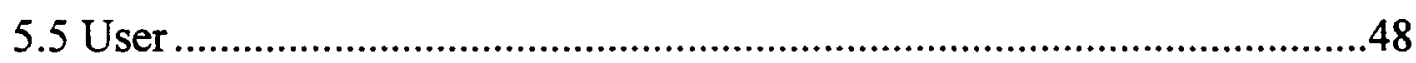

5.6 Role

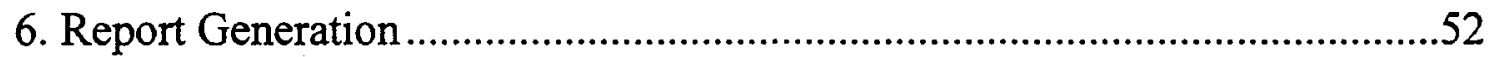

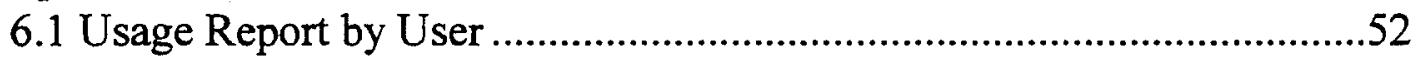

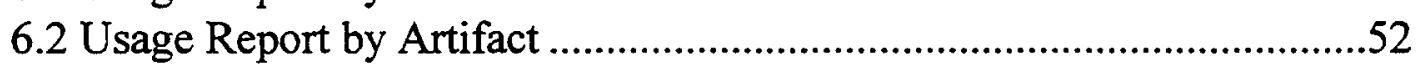

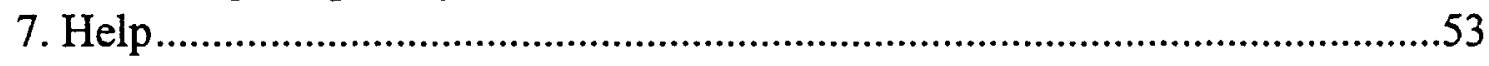

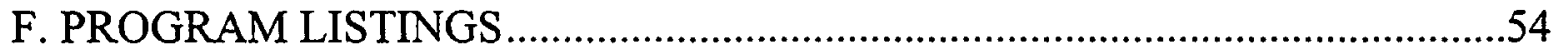




\section{LIST OF FIGURES}

Figure Page

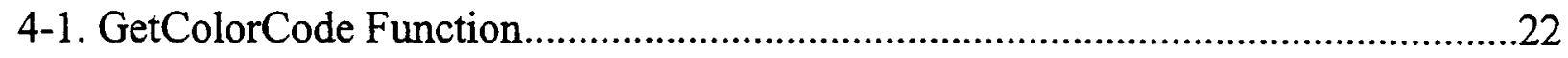

C-1. Entity-Relationship Diagram for the Reuse Tool .............................................30

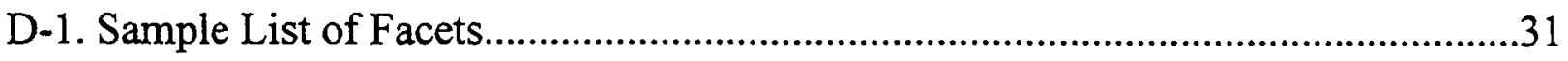

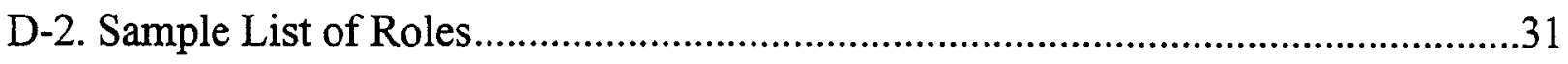

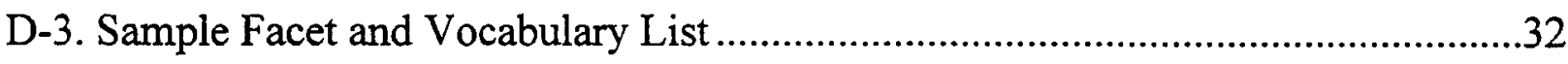

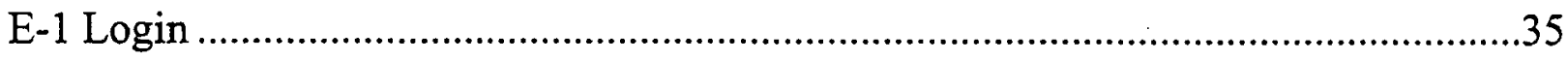

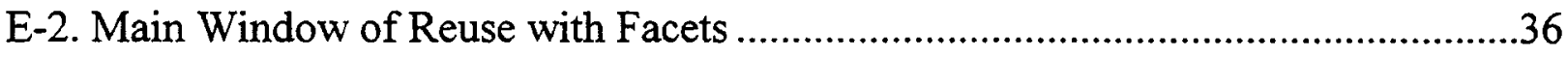

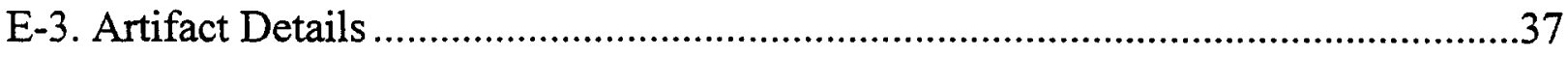

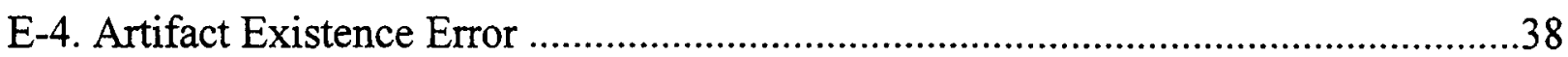

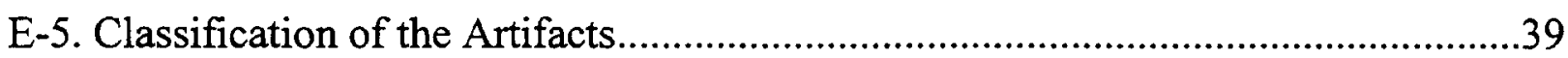

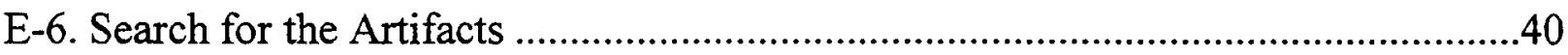

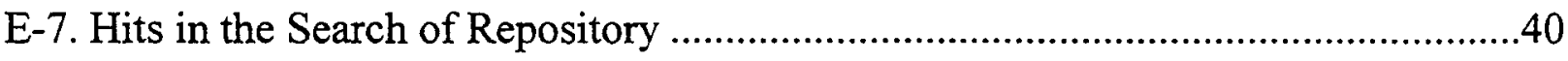

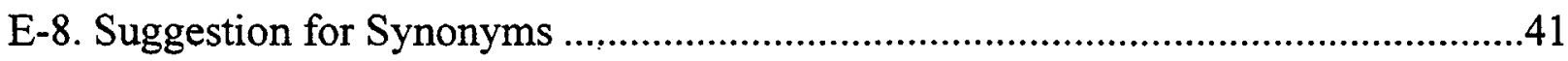

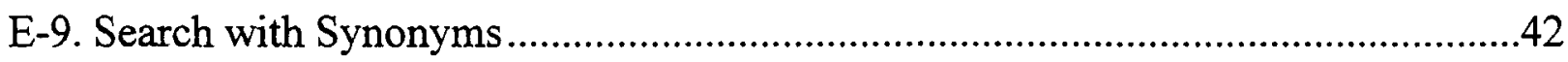

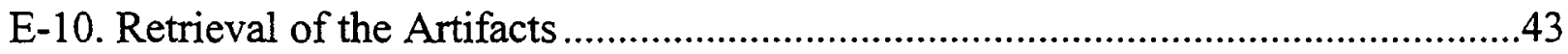

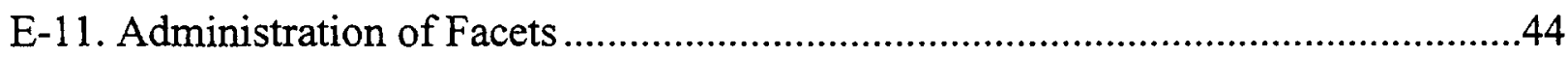

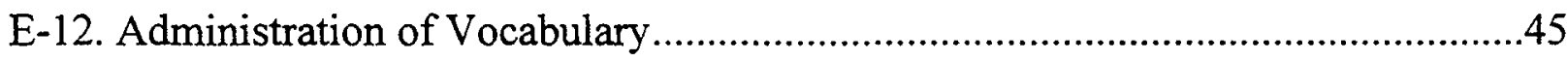




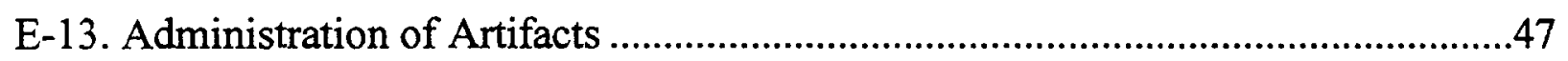

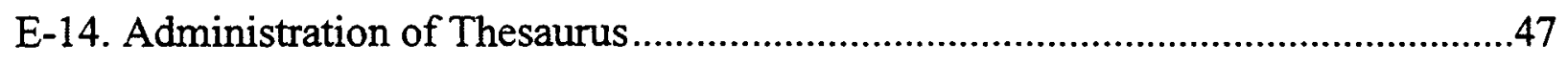

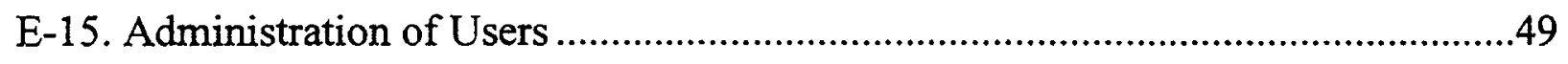

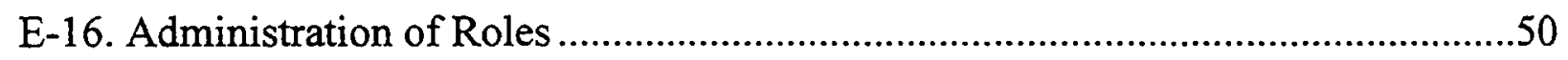

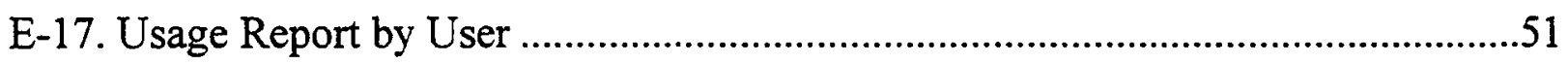

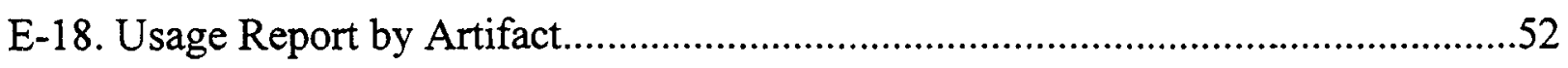

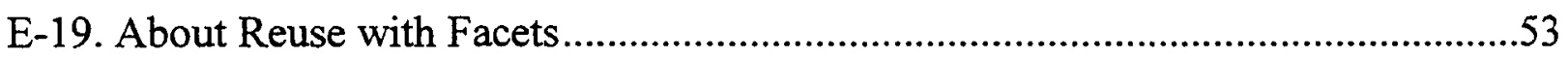




\section{CHAPTER I}

\section{INTRODUCTION}

Reusable software is widely believed to be the key to increased productivity in software development. While every "new" software system more often than not requires several components that have been developed before, these components frequently are redeveloped for the current system. Developers will be able to concentrate on developing truly new components if they could easily integrate past developments into their current one. Even though the strategy of reusability offers great promise, it has been generally unfulfilled to a great extent [Biggerstaff87] [Hall92].

The beginnings of software reuse can be traced back to the 1968 NATO Software Engineering Conference which focused on building large, reliable software systems in a cost effective way [Krueger92]. Recent renewed interest in software reuse has a lot to do with the cost of developing software. With the cost of powerful hardware falling everyday, ambitious and more powerful software systems are being planned to provide solutions for various practical problems. Quality along with productivity will improve in building these systems, if well-tested and already developed components were to be used in constructing these systems.

One of the fundamental problems in reusing software is the storage, search strategy, and retrieval of the components that are required for building systems. Whenever a component is built, a library at a central location should be available for keeping this component for retrieval at a later time. For both these purposes, a classification system should be available which, when incorporated into a tool, will facilitate the storage and retrieval of software components. 
As the main objective of this thesis, a tool was developed to help in reusing the existing software components. This tool uses the faceted classification system in organizing the software components.

Chapter II of this thesis reviews the current literature on software reuse. It also defines some of the basic terms used in software reuse, details the past and present efforts in software reuse, and also provides the basic background on software reuse. Part of this literature review discusses the faceted classification scheme, which is the basis for the tool developed as a part of the thesis. Since the database requirements are basically relational, the theory and description of the relational design is also briefly described in this chapter. Chapters III and IV discuss the tool in detail. Details about the implementation platform, design and implementation of this tool, and a sample reuse case are presented in these two chapters. Chapter VII summarizes the effort and briefly mentions the scope of future work in this area. Appendix $E$ has user and system administrator guide that explains how to use this tool. All functionality is clearly explained in this guide. All the source code is attached as the Appendix F. 


\section{CHAPTER II}

\section{LITERATURE REVIEW}

\subsection{Definitions}

This section contains some basic definitions of software reuse that are used in this thesis. Most of these definitions are taken from two main references [Krueger92] [PrietoDiaz91].

Software reuse can be defined as using the existing software artifacts in building a new software system. A software artifact can be a source code fragment, a design structure, a module-level implementation structure, a specification, a documentation item, a transformation, etc.

Abstraction refers to hiding the implementation details of source code and providing only the necessary information about the functionality of that source code. Abstraction is an important feature of software reuse. Without abstraction, the reuser will be forced to spend a lot of time trying to understand what each artifact is doing and how it can be reused.

Selection refers to choosing a particular software artifact from a collection according to the needs of the reuser. If a classification system is used to organize the collection, the selection process will be greatly simplified.

Specialization is transforming an existing software artifact to meet the current needs. This specialization is achieved through parameters, constraints, and other forms of refinement. 
Integration refers to adding an existing software artifact to the system that is currently being developed. Module interconnection languages are examples of integration frameworks.

The faceted classification system is a scheme for organization based on facets or attributes of objects. A facet list is a collection of terms that can identify or describe various parts of a facet of the domain. A facet list can also be referred to as the vocabulary for classifying software artifacts.

The relational model refers to organizing data elements through tables and tables only. With the help of common columns amongst the tables, relationships are defined among different tables.

\subsection{Software Reuse}

\subsubsection{History and Past Research Efforts}

As mentioned in the introduction, a historically important conference in the field of software reuse was the 1968 NATO Software Engineering Conference that was held in Garmisch, Germany. But the origins of software reuse can be traced back to as early as 1949 when the University of Cambridge proposed the first subroutine library on its stored program computer EDSAC [Tracz88a].

A more recent event in the field of reuse is applying it to alleviate the "Software Crisis" in the context of the development of the Ada programming language. This language was developed under the auspices of the US Department of Defense (DoD) in the early 80 's. Software reuse and the object-oriented approach are two of the aspects of program development that Ada's features were expected to support [Tracz87].

Ada has several language constructs to facilitate the development of reusable components. These include a package construct, which separates specification and implementation, and an overload resolution construct, which facilitates the semantic and syntactic reuse of functions. Ada also enforces strong typing. The Department of Defense 
directives on standardization, validation, and mandated use of Ada has enhanced the chances of software reuse making a significant impact on software crisis at DoD.

A large number of corporations also have contributed to the field of software reuse. These corporations include Boeing, Ford, General Dynamics, IBM, Lockheed, and Honeywell. They have projects addressing several aspects of reuse such as standardization of support tools, construction of libraries, automated catalog and library interaction, and coding without language or implementation tricks [Tracz87].

\subsubsection{Current Practice}

As discussed in the previous section, the concept of software reuse has been in practice for some time. The following subsections detail the different ways this concept is practiced [Krueger92] [Hall87] [Zand94] [Zand93] [Zand92] [Swanson92].

2.2.2.1 Vertical Reuse or High-Level Languages. Reuse in languages started with assembly languages. Assembly language routines provided an abstraction to the machine language routines for most of the hardware-level functions. In the newer languages, libraries of standard functions further extend this abstraction. Object-oriented languages such as $\mathrm{CH}$ and Smalltalk provide powerful features for the user to extend such abstractions. When the concept of abstraction is further extended, we get Very HighLevel Languages (VHLL). These languages resemble application generators in the sense that the specification for a certain task is automatically transformed into executable systems. This property derives another name for VHLLs - executable specification languages.

2.2.2.2 Horizontal Reuse. Within a level of abstraction, functions can be reused. Pipes and filters of UNIX facilitate reuse under this category. In this case, the output of one function is transferred to another function; in effect reusing the second function without having to rewrite it. Batch processing also comes under this category. Horizontal reuse is 
different from vertical reuse in the sense that the first category uses high-level languages whereas the second category uses the operating system functionality.

2.2.2.3 Design and Code Scavenging. Sometimes programmers scavenge code and designs from a previously developed systems. Typically, the locations of this type of artifact and the concepts used reside primarily in the programmers' head. So, recalling from memory, a programmer will copy code fragments from an old system and integrate them into the current project. This type of reuse becomes more effective as the programmer becomes more experienced.

2.2.2.4 Source Code Components. Similar to the hardware component industry, this notion suggests an industry of off-the-shelf source code components. Ideally, functions with clearly defined input and output values can be manufactured for use in several different programs. The packaging of these components can in principle use systematic techniques such as catalogs and libraries of components. To use these functions, a reuser can set the parameters rather than editing the source code directly, even with the availability of source code in such component libraries.

2.2.2.5 Application Generators. For application generators, the input is the specification of the required task and the output is the executable program that addresses the required task. These are similar to conventional programming language compilers, except that they are at a higher level. Application generators typically focus on a narrow domain and generate code to solve the problems in that area only. Application generators work at such a high level that they concentrate on what the system should do rather than how it is done. So, algorithms and data structures are automatically generated for the reuser. Application generators have been developed in several domains including database management, textual report generation, and graphical report generation [Burton87]. 
Parsers and compiler generators like lex and yacc come under this category. Lex addresses lexical analysis and yacc deals with parsing.

2.2.2.6 Software Schemas. In software schemas, the notion is reusing algorithms and data structures rather than the code itself. An example of this schema is the PARIS system [Katz87]. In this system, a reuser starts the development by giving a problem statement, which is a formal set of computational requirements. Then, with its sophisticated retrieval system, PARIS supplies the schema that satisfies the problem statement.

\subsubsection{Reuse Classification Criteria}

Reusability has three important aspects: the abstraction level, customization methods, and reusability conditions [Lenz87] [Krueger92]. A classification system used for software should facilitate all these three aspects of reuse.

2.2.3.1 Abstraction Level. Abstraction is the most important feature of software reuse. If the implementation details of the software artifact are not hidden, a programmer will be forced to spend more time in understanding the internals. The comprehension overhead may eventually force the programmer to redevelop that software artifact instead of reusing it. A reasonable amount of abstraction should be practiced in all units of software, be they specifications, designs, or code.

2.2.3.2 Customization Methods. Once a software component is identified as a candidate to be used in a new system, it has to be tailored to meet the current needs. This can be done by simple parameterization or by changing of the internals. When a candidate artifact needs no tailoring at all, obviously we have the best form of software reuse.

2.2.3.3 Reusability Conditions. When the domain, in which the software artifacts are being developed, reaches a certain degree of maturity, concepts used in that domain become apparent. This paves the way for higher software reuse. In a new domain, 
reusability becomes difficult because of the lack of software artifacts and the lack of domain knowledge by the programmer. So, a thorough analysis of the domain will help improve software reuse.

\subsubsection{Economics of Reuse}

As mentioned in the introduction, the chief reason behind the increasing interest in software reuse is the enormous increase in the cost of developing complex software. A simple cost model proposed by John Gaffney of the Software Productivity Consortium is as follows [Tracz88b] [Barnes87] :

$$
C=(1-R)^{*} 1+b^{*} R
$$

where $C$ is cost of developing software, $R$ is the percentage of code reused, and $b$ is the cost of reusing a line of code / cost to develop a new line of code.

As we can see from the above model, savings will be more when more code is reused or when the development of new code is higher than reusing the existing code. But all these gains, that can be realized from the reuse of software, are moderated by some organizational factors. Project managers in large organizations are not typically rewarded for the reuse of old software. Without organizational commitment, it becomes difficult to develop and maintain libraries with reuse in mind [Barnes87].

\subsection{Faceted Classification System}

An important aspect of software reuse is the organization of software artifacts so that they can be searched and retrieved easily. This organization methodology also should provide an easy way of inserting newly developed artifacts into the collection.

Three concepts of organization are widely used throughout conventional libraries in the world. The first one is the Dewey Decimal System, which is used in a number of libraries in the United States, the second one is the Library of Congress Classification System, which is used by the Library of Congress and several other libraries in the United 
States, and the third one is the Faceted Classification System, which is widely used in Europe and India [Prieto-Diaz91]. In the Dewey Classification System, the possible classes are predefined. Hence, when a title needs to be classified under this system, the librarian should find a class that best fits the title. In order to do this, the librarian should have expertise in both the Dewey Classification System and the subject matter that the title represents. As expected, with several closely related classes, a title spanning across several classes becomes difficult to classify.

The Library of Congress (LC) System is similar to the Dewey Classification System in many respects, especially in terms of the predefined classes. But its notation differs significantly from Dewey. The following example, from [Immorth71], explains the difference. The third edition of Richard D. Altick and Andrew Wright's Selective Bibliography for the Study of English and American Literature has a call number Z2011.A4 1967 under the Library of Congress System, and 016.82 A468s 1967 under the Dewey Decimal system. Each of these call numbers has three components: Class Number (Z2011 in LC and 016.82 in Dewey), Author Number (A4 in LC and A468s in Dewey), and Publication Date (1967). Z2011 and 016.82 are predefined classes in both of these systems.

With the Faceted Classification System, it becomes easy to decide on titles spanning across several classes. A faceted scheme contains as many facet lists as the domain to be classified requires. Each facet list contains as many keywords as needed to describe that facet. So, when a librarian wants to classify a particular title under this system, the librarian will select a term from each facet that suits the title. After all the terms are selected, the class that is arrived at will be the best fit for this title, hence offering flexibility and accuracy.

Another recent approach to information retrieval is by free text analysis [PrietoDiaz91]. There are several reasons why free text analysis does not work for the analysis of source code functions. First of all, there might not be a lot of free text in the source 
code - unless the programmer decides to do a lot of commenting. Variable naming conventions can differ from programmer to programmer and also from function to function. In effect, by free text analysis of a function, it is not in general possible to find out what a function is doing or how it is doing it. In contrast, in the Faceted Classification System, there are predefined lists of key words. This list of key words or standard vocabulary can be updated as needed. Hence this classification not only provides the flexibility of having a reasonable number of key words, but it also provides consistency among the programmers or reusers and the librarians of a reuse system [Prieto-Diaz91].

\subsubsection{Facets and Vocabulary}

Figure D-3 contains the list of facets and vocabulary that is developed for the PC applications domain. For the purpose of the Faceted Classification System, a total of seven facets are suggested to characterize this domain (this system provides the flexibility of addition, deletion, and modification to these facets at any time). These seven facets are the Operating System for which the software artifact works, the Language in which the artifact is developed, the part of the system where this artifact works best, the action or service this artifact provides, a name given to this artifact by the developer, the name of the author, and the date of creation.

Each of these facets has a list of choices (or vocabulary) to further describe the software artifact. This vocabulary can also be modified as the repository of artifacts grows. The following paragraphs briefly describe the vocabulary for each of these facets.

In a large corporation, normally several operating systems are used on the microcomputer domain. They include DOS, Windows, Windows NT, OS/2, and Unix. Others may be added as they become available. Similarly, several languages might be used in the development process, they include $\mathrm{C} / \mathrm{C}++$, Pascal, and FORTRAN. 
In the case of operating systems and languages, versions also play an important role. For example, DOS can be further refined into DOS3, DOS4, DOS5, and DOS6. The facets, vocabulary, and files related to the older artifacts can be deleted to make room for the newer ones.

The two facets refined to as system component and action describe the functionality of the artifact. The system component suggests the place where this artifact might be used. The action suggests further specialization of the artifact that is under consideration.

The last three facets deal with the creation of the artifact. The Name given to it by the author, the name of the author, and the date of creation make up this set. Since these are important to identify the artifact, they are also stored in the artifact table of the database.

\subsection{Relational Database Management}

The relational model refers to a database system that contains tables and nothing but tables at the logical level for organizing data [Date91]. There might also be relations among these tables to connect the data among several tables. So, a user conceptualizes the whole data as tables and relations. Each table contains rows and columns. Each row, or a record from a table, is the complete description of one entity. This entity can be a person, a sale, etc. Each entity is described by several fields, characteristics, or attributes. These fields are represented by columns [MS-SQL93].

SQL (Structured Query Language) is the standard language (with various dialects) that implements the relational database model. This high-level language was originally developed at IBM in the mid-1970s. This language includes statements not only for querying and retrieving data from a database, but also for creating new databases, and modifying and updating them. The American National Standards Institute (ANSI) recently came up with a standard SQL language called Transact-SQL [Transact-SQL93]. 
The relational model consists of three phases. The first phase in the relational model is data organization. In this phase, the specification of the system is collected in terms of tables, i.e., the rows and columns in each table. Here the primary and foreign keys for each table are also defined. A primary key is one or more columns that uniquely identify the rows or records in the table. A foreign key is a column in a table that is similar to a column in another table. With the help of foreign keys, two or more tables can be joined. The second phase is data definition. In this phase, storage is allocated to the database and then tables are created. So, this phase mainly creates the holders for data. The third phase is data manipulation. In this phase, the functions required for data retrieval and data modification are developed. In the SQL language, data retrieval is primarily done by the SELECT statement, and data modification is done by INSERT, UPDATE, and DELETE statements. As the meanings suggest, to insert new data values into the tables, the INSERT statement is used, to modify the currently existing data, the UPDATE command is used, and to delete any data from the database, the DELETE statement is used.

The above three phases constitute the basic database operations. There are also some additional facilities provided for easy access to data and to keep the integrity of the data [Transact-SQL93]. Indexes can be defined on the tables to access data quickly. There are basically four types of indexes: composite, unique, non-clustered, and clustered [MSSQL93]. A composite index is created on single or multiple columns. A unique index is created on single or multiple columns, where these columns make a unique key. When a non-clustered index is created, the data is not ordered physically. With the clustered index the data is physically ordered. On any table, there can be as many composite, unique, and non-clustered indexes as needed, but there can be only one clustered index, since this index physically orders the data [MS-SQL93]. It also should be noted that, when multiple indexes are created, the clustered index should be created first. 
Defaults, rules, and views are also part of the useful features of the relational model. Default values can be placed in the data holders when no value is specified. When a certain data is entered to be placed in the table, the rules check for the validity of that data for that column in terms of data types. Views help in presenting parts of a full table or database [MS-SQL93].

\subsubsection{Entity-Relationship Diagram for the Tool}

All elements required for reuse are stored using the relational model. Several interconnected tables are used in this model. Appendix $\mathrm{C}$ contains a diagram that represents the entity-relationship diagram for this tool. All the objects that makeup this database are described in the section 3.2 


\section{CHAPTER III \\ DESIGN AND IMPLEMENTATION ISSUES}

\subsection{Implementation Platform and Environment}

The tool was implemented on an IBM compatible personal computer running MSDOS version 6.0 operating system with the MS-Windows graphical environment. The IBM compatible personal computer was chosen because of its widespread availability, and MS-DOS is arguably the standard operating system for personal computers. MSWindows environment was chosen to develop the Graphical User Interface (GUI) for this application.

\subsubsection{Personal Computers Today}

Presently there are two major standards for personal computers - the IBM compatible personal computers and the Macintosh line of personal computers. Recently, in years 1993 and 1994, personal computers based on other CPUs have also been introduced. These computers include PowerPCs and PowerMacs based on the RISC processor jointly developed by IBM, Apple, and Motorola. DEC also introduced a new range of personal computers based on its alpha chip.

The term PC normally refers to the IBM compatible personal computers. The first PCs were developed by IBM with the Intel 8086 processor. This processor has gone through several generations from 8086 to 80286 to 80386 to 80486 . The latest generation of this processor is called Pentium (or 80586) which was introduced by Intel in 1993. Presently, the standards for the PC are jointly set by Intel, Microsoft, and several leading vendors of PCs. Since the introduction of the PC in 1981, its power has increased tremendously. Original PCs could handle a maximum of one megabyte of memory, but 
they were shipped with far less. Today eight to sixteen megabytes of memory is normally available on most of the new PC's. There has been an attendant increase in the size of the hard disk, and the clarity and the graphics capability of the display also.

\subsubsection{DOS Operating System}

IBM contracted the Microsoft Corporation to write an operating system for its introductory personal computer, simply called IBM PC. It is estimated that presently DOS runs on one hundred million personal computers world wide. DOS is available in several flavors. The operating system published by Microsoft is called MS-DOS, the one published by Novell (originally by Digital Research) is called DR-DOS, and the IBM product for the operating system is called PC-DOS. Currently the MS-DOS is at Version 6.2 .

DOS is a text based operating system with limited support for graphics. The interface of the DOS itself is character based. Several utilities and programs provide the graphics and multi-media capability to the DOS operating system. Version $6 . x$ will be the last version to have the character based interface. One version of DOS from Microsoft has the widely-accepted user interface of Microsoft Windows.

\subsubsection{Windows Graphical Environment}

Microsoft Windows is a graphical operating environment designed to run on the DOS operating system. Microsoft did not have much success with the graphical interfaces provided by the Windows Versions 1.x and 2.x. One of the chief reasons for this failure was the lack of powerful hardware at the time of their release. But when Microsoft Windows Version 3.0 was released in 1990, its user interface was an instant success. Version 3.1 released in 1992 provided several other capabilities such as multi-media support, object linking, and embedding.

In 1993, Microsoft released a high-end operating system called Windows NT. This is not only a regular operating system (unlike Windows, which requires DOS as the 
underlying operating system), but also has several other capabilities such as networking and security. Windows NT, however, retains the user interface of the Windows operating environment.

\subsection{Specification and Design of the Tool}

The tool that was developed as part of this thesis is named as Reuse with Facets. This tool can be divided into two major parts: the User Interface and the Database.

\subsubsection{User Interface}

The user interface of the Reuse with Facets tool consists of all the dialog boxes that take the information from the user and present the results. Detailed explanation about the individual dialog boxes and the functionality associated with them is provided in Appendix E titled User and System Administrator Guide. The following paragraphs summarize the user interface provided with the tool.

When this tool, Reuse with Facets, is started by double-clicking the icon provided in the Program Manager, it comes up with a main window titled Reuse with Facets. This window has several menus near the top border, with each menu containing one or more commands. The menus provided here are User, Librarian, Reports, and Help. The User menu has two commands, Retrieval and Insertion. The Librarian menu has five commands named Facet, Vocabulary, Artifact, User, and Role. The Reports menu has two commands, User Report and Artifact Report. Finally, the Help menu contains a command to invoke an "about" box that gives the version-related information about the tool, this also provides the user's general system related information.

Each of these menus evokes a form or a dialog box. Each dialog box has text boxes, list boxes, option buttons, command buttons, and text or graphical instructions to facilitate input from users. Some dialog boxes have tables or grids to present the information to users. The user interface used in this tool conforms to the Microsoft 
Windows 3.1 standards and is consistent across the dialog boxes. As mentioned above, all these dialog boxes are thoroughly explained in the user's guide provided in Appendix E.

\subsubsection{Database}

The database part of the Reuse with Facets tool is a relational database. All the elements required for reuse are stored using the relational model. Several interconnected tables are used in this model. The diagram in Appendix C (titled Relational Schema) represents the entity-relationship diagram for this tool. The following discussion further elaborates the tables used which are tables for Users, Role, Action, Event, Search Results, Facet, Vocabulary, Thesaurus, Artifact.

All the information related to the users is stored in the Users table. Each entry in this table can be uniquely identified with the help of UserId. The RoleId field in this table identifies the title or role of the user in this system. The different roles a user can take are discussed under the Role table. Further, this table stores the First Name, Last Name, Address, and the Telephone Number of users. A comments field is also provided to facilitate additional comments on the user-related information.

The Role Table facilitates the storage of different titles or roles a user can take. Initially, three default roles are provided. Additional roles can be added as the requirement arises. The default roles are: Developer, Manager, and Librarian. The Developer produces and reuses the software artifacts. In general, programmers assume both the roles of developing and reusing. If a situation exists where these two roles are separated, an additional role can be created in the system. The role of Manager is suggested for the person who oversees the complete development process. Managers generally will not produce or reuse the software artifacts, rather they will be responsible for the development at a higher level. And finally, the Librarian is responsible for the maintenance of this system. This role can also be termed as the Super User. The Librarian will constantly update and refine the vocabulary and monitor reuse in the system. The 
tool will provide a default user with the role of Librarian, who will work on the further expansion of the system. Hence, this role cannot be deleted from the system.

The Action Table is used to store the names and the descriptions of the actions that a particular user can perform. Three default actions are provided with the tool. The tool provides facilities to add or delete actions. The three default actions that are possible for the reuse events are inserting new software artifacts, retrieving the software artifacts for reuse, and general browsing. The correlation between these actions and the roles mentioned in the above paragraph for a typical situation are: Librarian classifies and inserts the new software artifacts, Developer/Reuser retrieves the existing software artifacts from the system, Manager does the general browsing to get an idea of the existing collection of software artifacts and the amount of reuse.

The Event Table provides a place holder for the information about individual reuse events occurring in the system. Each event can be uniquely identified with the help of an EventId. All the elements that make up an event, i.e., the user of the system, the role of this user, the action this user performed, the string of facet ids and the vocabulary associated with each facet, the result this search operation has ended up with, the artifact involved in the search, the date and time of the event, and any comments about the event, are stored in this table. All these events can be summarized later in the form of reports. These reports provide a number of software performance and usage metrics about the system.

The SearchResult table contains all the possible outcomes of the search for an artifact. These outcomes are Found, Not Found, or Aborted. Aborted is reported for a search result when the user closes the system or the query while the search is still being performed.

The Facet and Vocabulary tables make up the necessary words required for the classification of software artifacts. The tool provides several default facets and vocabulary for the PC applications domain. The Facet table is the holder of the name of 
the facet and a description associated with it. The Vocabulary Table provides the storage for the different words that describe each facet. Since a vocabulary word is associated with a particular facet, a Facetld field is also added to this field.

The Thesaurus table can be used to obtain synonyms for the vocabulary used for the artifacts in the system. Even though this table provides synonyms for the vocabulary mentioned in the previous table, these two tables are separate. The reason for this separation is that a word or synonym still remains in the system even after a particular facet or vocabulary is removed from the system. This ensures the growth of the thesaurus.

The Artifact table stores the classification and the physical details of the artifact. The FacetId string and the Vocabularyld string give the classification. ArtifactName, ArtifactFileName, ArtifactPath, ArtifactSize, ArtifactDescription, ArtifactAuthor, and ArtifactDateOfInsertion provide the physical details of the system.

\subsection{Development and Implementation of the Tool}

The graphical interface for this application is developed in Microsoft Visual Basic Version 3.0. The relational database which holds the information and classification about the reusable software artifacts is developed using on Microsoft Access Version 1.1 database engine. For this database development, more sophisticated database environments such as the MS-SQL Server were considered. Since the SQL Server does not provide executables (i.e., the SQL Server should be present on the computer on which an application is running), Microsoft Access was given priority as the database engine. Also, Microsoft Access 1.1 is fully integrated into Microsoft Visual Basic 3.0 for all its database features.

A setup program was also developed to ease the setting up of this tool on a reuser's workstation. This tool installs a default database which can be moved to a network directory after the setup. Step-by-step instructions are provided in the user's 
guide presented under Appendix E. This tool was tested for insertion and retrieval of software artifacts. It was also tested for all the system administration functions (or the Librarian functions) concerning updating the list of facets, vocabulary, artifacts, thesaurus, users, and roles.

Code for this program is broken in to several forms and module files. Each form contains the instance code for all the user interface elements like command buttons, menus, and the form appearance itself. As this is event driven programming, each user interface element contains the code module that is to be executed when certain event occurs. Examples for an event are mouse click, mouse double click, pressing a key, etc. All the form functions are local to that form, where as module files contain global functions.

All the functions in this program contain a header. Comments are intermixed with the code to make it readable. 


\section{CHAPTER IV}

\section{TESTING OF THE TOOL}

\subsection{Sample Reuse Done with the Tool}

This chapter briefly outlines a sample deposit and retrieval. A number of observations are also mentioned. Figure 4-1 depicts a function that changes the string of a color name to a hexadecimal color code. This function can be used to obtain a color name from the user and then generate the color code to actually use that color. So, this is mainly a user interface related function. And further, this is a color related function.

This function is written in Visual Basic and runs under the Windows operating system. The Name of this function is GetColorCode and is written by Mareddy on 01/01/94. So the class for this function according to the Faceted Classification System is Windows-VB-UserInterface-Color-GetColorCode-Mareddy-010194. This name is obtained by the synthesis of all the facets for the function.

For the retrieval of this component, the same class (with most of the facets) is inputted into the system. For example, if the Action facet for this artifact is selected as "Shade" instead of "Color", the system will miss this artifact. But in the system Shade is also defined as a synonym for color. So, a further prompt will be given to the reuser, asking whether to look for classes for synonymous facets. Subsequently, the tool can retrieve the above artifact. 


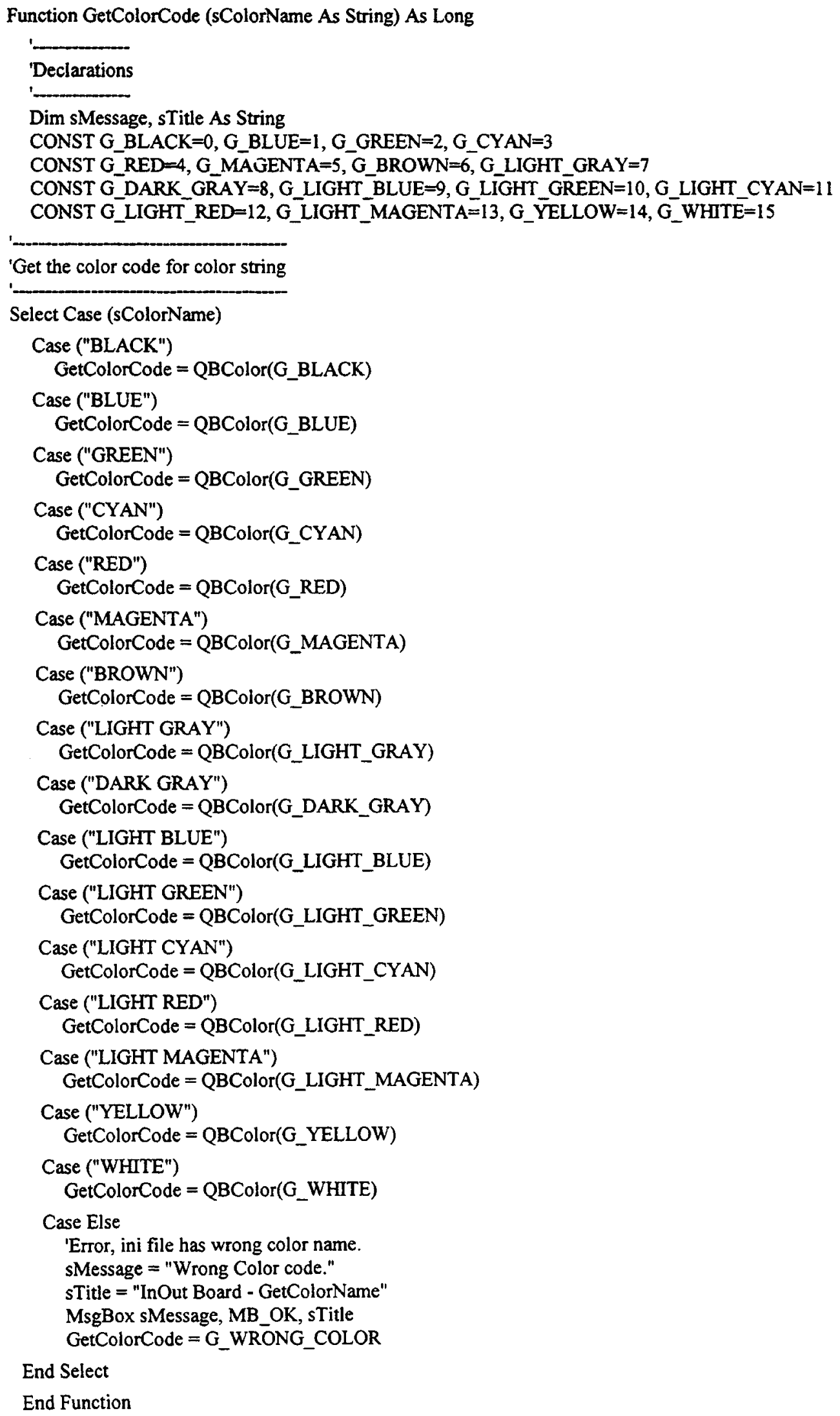




\subsection{Observations}

Facets.

The following observations were made during the testing of this tool, Reuse with

- Initially, the number of hits for a sample class were few. The reason for this was that the number of artifacts present in the system was small. As the repository of software artifacts grew, the chance of retrieving an artifact with desired classification increased.

- This author depended heavily on memory for reusing the existing artifact, in terms of this locations and names. But now, a convenient system, i.e., the Reuse with Facets tool, existed with a classification system and a friendly user interface.

- Reports provide a way to measure the amount of reuse happening in an organization. The functions that are not being used over a period of time can be deleted and popular artifacts can be left in the system for reuse.

- A new specification called OLE (Object Linking and Embedding) has some similarity with this tool. Microsoft Windows comes with a large set of functions called Windows API (Application Programming Interface). These functions let the programmer access the operating system related functionality. These functions are randomly named and grouped, making Windows API chaotic and hard to use. A new specification called OLE for personal computer operating systems defines the communication between objects. Objects adhering to this standard are called Windows Objects and will be able to communicate effectively. Each object will be able to access the functions of other objects without knowing the implementation details.

Some limitations were also observed regarding this tool.

- This tool works only in the Microsoft Windows environment. This tool is not readily portable to other operating systems.

- This tool is passive in identifying new artifacts, extracting new facets, and verification of the inputs.

- Conceptual distance between two facets is enforced through synonyms, rather than by assigning weights to terms. 


\section{CHAPTER V}

\section{SUMMARY, CONCLUSIONS, AND FUTURE WORK}

This thesis and the tool described mainly dealt with the implementation of the Faceted Classification System for reuse in the Personal Computer applications development domain. As part of this effort, a database schema, a list of facets, and a vocabulary pertinent to this domain were developed. The database developed conforms to the standard relational model.

This model of software reuse can be effectively used in a multitude of development situations. Pure software development corporations, with development projects in several platforms, can benefit the most from such a tool. But so will the Information Systems divisions of the corporations where software development is not the main business. They will be able to save time and resources with the help of an effective classification system and a reuse tool.

The success of reuse depends on how strong the organizational commitment is to reuse. In the case of the Faceted Classification System, it is equally important to develop and maintain a pertinent list of facets and vocabulary.

Future work to extend this thesis can be towards addressing the limitations mentioned at the end of Chapter III. A domain-independent tool can help the multiplatform development tremendously, thus increasing reuse across domains. More intelligence can also be built into the tool, thus facilitating the identification of artifacts and verification of inputs. Adding capabilities such as operators and composition rules to facilitate the fabrication of new software artifacts based on or by combining existing artifacts. 


\section{REFERENCES}

[Barnes87] B. Barnes, T. Durek, J. Gaffney, and A. Pyster, "A Framework and Economic Foundation for Software Reuse", Proceedings of the RMISE Workshop on Software Reuse, Rocky Mountain Institute of Software Engineering, Boulder, Colorado, pp. 77-88, October 1987.

[Biggerstaff87] T. Biggerstaff and C. Richter, "Reusability: Framework, Assessment, and Directions", IEEE Software, pp. 41-49, July 1987.

[Burton87] B.A. Burton, R.W. Aragon, S.A. Bailey, K.D. Koehler, and L.A. Mayes, "The Reusable Software Library", IEEE Software, pp. 25-33, July 1987.

[Date91] C.J. Date and H. Darwen, Relational Database Writings 1989-1991, AddisonWesley Publishing Company, Massachusetts, 1991.

[Hall87] P.A.V. Hall, "Software Components and Reuse - Getting More out of Your Code", The International Journal of Information and Software Technology, Vol. 29, No. 1, pp. 38-43, January/February 1987.

[Hall92] P.A.V. Hall, Software Reuse and Reverse Engineering in Practice, Chapman and Hall, New York, 1992.

[Immroth71] J.P. Immroth, $A$ Guide to the Library of Congress Classification, Libraries Unlimited, Colorado, 1971.

[Katz87] S. Katz, C.A. Richter, and K.S. The, "PARIS: A System for Reusing Partially Interpreted Schemas", Proceedings of the Ninth Annual International Conference on Software Engineering, Washington, D.C., pp. 377-385, March/April 1987.

[Krueger92] C. Krueger, "Software Reuse", ACM Computing Surveys, Vol. 24, No. 2, pp. 131-183, June 1992.

[Lenz87] M. Lenz, H.A. Schmid, and P.W. Wolf, "Software Reuse through Building Blocks", IEEE Software, pp. 34-42, July 1987.

[MS-SQL93] Microsoft SQL Server Implementation Notes, Microsoft University, 1993.

[Prieto-Diaz91] R. Prieto-Diaz, "Implementing Faceted Classification for Software Reuse", Communications of the ACM, Vol. 34, No. 5, pp. 89-97, May 1991.

[Swanson92] J. E. Swanson and Mansur H. Samadzadeh, "A Reusable Software Catalog Interface", Proceedings of the 1992 ACM/SIGAPP Symposium on Applied Computing (SAC'92), pp. 1076-1082, Kansas City, MO, March 1992. 
[Tracz87] W. Tracz, "Ada Reusability Efforts: A Survey of the State of the Practice", Proceedings of the Fifth Annual Joint Conference on Ada Technology and Washington Ada Symposium, U.S. Army Communications-Electronics Command, Ft. Monmouth, N.J. pp. 35-44, March 1987.

[Tracz88a] W. Tracz, "Software Reuse Myths", ACM SIGSOFT Software Engineering Notes, Vol. 13, No. 1, pp. 17-21, January 1988.

[Tracz88b] W. Tracz, "RMISE Workshop on Software Reuse: Meeting Summary", Tutorial on Software Reuse: Emerging Technology, Boulder, CO, pp. 41-53, October 1988.

[Transact-SQL93] Transact-SQL User's Guide for SQL Server, Microsoft Corporation, 1993.

[Zand92] M. K. Zand, Mansur H. Samadzadeh, H. Saiedian, and H. Farat, "Classification and Identification of Software Components", Proceedings of the Second Golden West International Conference on Intelligent Systems, pp. 275-280, Reno, NV, June 1992.

[Zand93] M. K. Zand, K. M. George, Mansur H. Samadzadeh, and H. Saiedian, "An Interconnection Language for Reuse at the Template/Module Level", The Journal of Systems and Software, Vol. 23, No. 1, pp. 9-26, October 1993.

[Zand93] M. K. Zand, Mansur H. Samadzadeh, and H. Saiedian, "Version Management for ROPCO: A Micro-Incremental Reuse Environment", The Journal of Information and Software Technology, Vol. 35, No. 11/12, pp. 627-637, November/December 1993. 


\section{APPENDIX A}

\section{GLOSSARY}

Ada

Client-Server Model

Column

Database Engine

Dewey Decimal System

Domain

Facet

Faceted Classification System
A programming language developed under the auspices of the US Department of Defense to alleviate the "Software Crisis".

In this model, a central computer holds the databases or files and there are several workstations connected to this computer that have access to those databases and files. The central computer is called the server and all the workstations are called clients.

Each column or field describes one characteristic of the entity (e.g., a person's name or address, a company's name or president, items sold, a quantity, or a date).

A program that facilitates the definition, development, and querying of a database. A typical database program contains two parts: a front end for the user interface and a back end for holding the database.

A classification system, mainly used in US libraries for organizing books. In this system, all classes are predefined.

An area or platform of development. For example, UNIX and DOS are two major platforms or domains for software development on mainframes and personal computers, respectively.

An attribute of a domain. For example, UNIX tools may be described by these four facets: by action, by object, by data structure, and by system.

A classification system that is widely used in the libraries of India and Europe. Here, classes are 
Foreign Key

Graphical User Interface (GUI)

Librarian

Pipes and Filters

Primary Key

Relational Model

Reuser

Row

SQL

Table obtained from various faceted lists for a particular domain.

A column that provides a link with another table. These two columns should be of identical data types.

A non-character based interface to a programming application. Commands to the program are typically given through menus, buttons, dialog boxes, and other graphical elements.

A person organizing and maintaining the software artifacts that will be used in future software projects.

Inputting one program's output to another program, where two programs are on the same command line with a pipe $(\mid)$ in between. Filter commands divide, rearrange, or extract portions of the information that passes through them (for e.g., more, find, and sort)

The column or group of columns whose values uniquely identify each row in the table.

A data organization and retrieval model first proposed by Dr. E. F. Codd in 1960's. This model uses tables and only tables at the logical level to organize data.

A programmer building a software system who uses software artifacts from a previously developed system.

Each row or record of a table describes one occurrence of an entity (e.g., a person, a company, or a sale).

Structured Query Language, a language originally developed at IBM in 1970's based on the relational model.

Tables are logical data structures in the relational system. Each table contains rows and columns. 


\section{APPENDIX B}

\section{TRADEMARK INFORMATION}

IBM

MS-DOS

MS-Windows

MS-Visual Basic

MS-Access

MS-SQL Server

DEC

MacIntosh

Motorola

IBM is the registered trademark of International Business Machines.

MS-DOS is the registered trademark of Microsoft Corporation.

MS-Windows is the registered trademark of Microsoft Corporation.

MS-Visual Basic is the registered trademark of Microsoft Corporation.

MS-Access is the registered trademark of Microsoft Corporation.

MS-SQL Server is the registered trademark of Microsoft Corporation.

DEC is a registered trademark of Digital Equipment Corporation.

MacIntosh is a registered trademark of Apple Computer Corporation.

Motorola is a registered trademark of Motorola Corporation. 
APPENDIX C

RELATIONAL SCHEMA

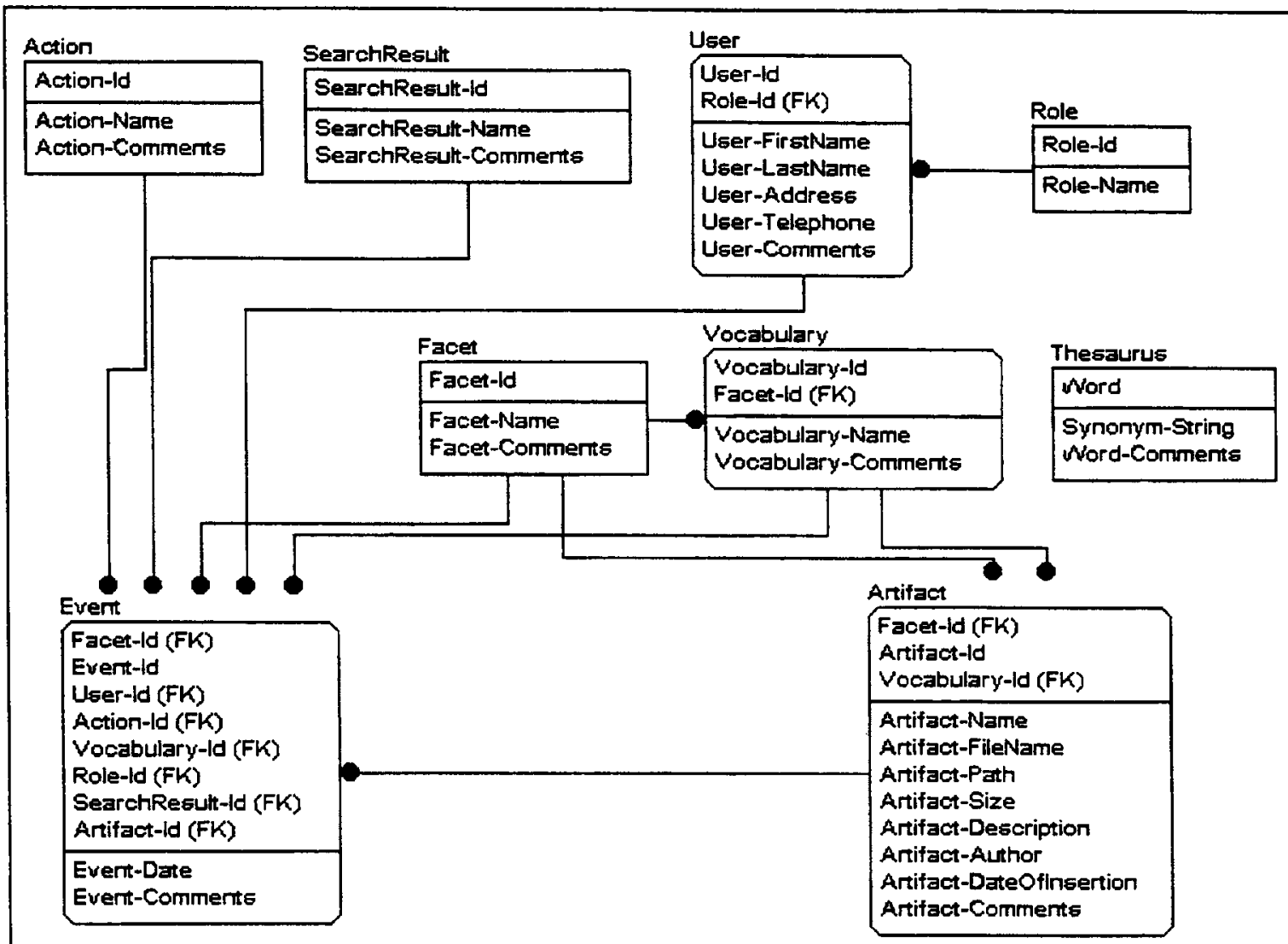

Figure C-1. Entity-Relationship Diagram for the Reuse with Facets tool 
APPENDIX D

SAMPLE DATABASE

\begin{tabular}{|c|c|}
\hline Number & Name of the Facet \\
\hline 1 & Operating System \\
\hline 2 & Language \\
\hline 3 & System Component \\
\hline 4 & Action \\
\hline 5 & Name \\
\hline 6 & Author \\
\hline 7 & Date \\
\hline
\end{tabular}

Figure D-1. Sample List of Facets

\begin{tabular}{|c|c|}
\hline Number & Name of the Role \\
\hline 1 & Developer/Reuser \\
\hline 2 & Librarian \\
\hline 3 & Manager \\
\hline
\end{tabular}

Figure D-2. Sample List of Roles 


\begin{tabular}{|c|c|c|c|c|c|c|}
\hline $\begin{array}{l}\text { Operating } \\
\text { System }\end{array}$ & Language & $\begin{array}{l}\text { System } \\
\text { Component }\end{array}$ & Action & Name & Author & Date \\
\hline DOS & $\mathrm{C}$ & Specificatio & Average & & & \\
\hline Windows & $\mathrm{C}++$ & $\mathrm{n}$ & Count & & & \\
\hline Windows & Pascal & Data Model & Color & & & \\
\hline NT & FORTRAN & User & Shade & & & \\
\hline $\mathrm{OS} / 2$ & VB & Interface & $\operatorname{Max}$ & & & \\
\hline UNIX & QB & Editor & Min & & & \\
\hline None & COBOL & Financial & Sum & & & \\
\hline \multirow[t]{12}{*}{ All } & Executable & Database & Len & & & \\
\hline & None & Initialization & Lower & & & \\
\hline & All & Formatting & Upper & & & \\
\hline & & Date \& & Cos & & & \\
\hline & & Time & $\operatorname{Sin}$ & & & \\
\hline & & Math \& Trig & Tan & & & \\
\hline & & Statistical & Create & & & \\
\hline & & Text & Insert & & & \\
\hline & & Logical & Load & & & \\
\hline & & Engineering & None & & & \\
\hline & & None & All & & & \\
\hline & & All & & & & \\
\hline
\end{tabular}

Figure D-3. Sample Facet and Vocabulary List 


\section{APPENDIX E \\ USER AND SYSTEM ADMINISTRATOR GUIDE}

\section{Introduction}

Welcome to Reuse with Facets, an effective tool to promote the reuse of software artifacts in your organization. This tool uses the faceted classification system and an intuitive user interface to facilitate the organization and retrieval of software artifacts created by your programmers and software development personnel. The following sections describe the setup and various features of the tool. This guide is prepared to address the needs of both the Librarian and the Developer. Since the functions of the Developer (as compared to the functions of the Librarian) do not include the classification-related tasks and the generation of reports for the purpose of software performance and usage metrics, user may refer to the sections related to the retrieval of software artifacts only.

\section{Setting up}

The Reuse with Facets tool can be installed on your system by using the program setup.exe. The setup program installs on your hard disk the main interface program, the database with the default facets, the vocabulary, roles, search results, the thesaurus, and other system related variables. Since this tool is written for Microsoft Windows, it installs several system related files on your hard disk. Also, since this tool is developed for reuse in PC applications development, all these defaults reflect the PC development domain. 


\subsection{Hardware and Software Requirements}

Before you install Reuse with Facets, make sure your computer meets the following minimum requirements in terms of hardware and software.

- Any IBM-compatible machine with an 80386 or higher processor.

- A hard disk with at least $2 \mathrm{MB}$ of free space. More disk space will be required as the collection of software artifacts grows.

- A $51 / 4$ or $31 / 2$ inch floppy disk drive.

- A VGA monitor or better.

- Two megabytes of memory (four megabytes recommended for speed).

- A network. Even though this tool works for a single-user setup, when multiple people are involved in the software development process, a network and a central server to store the software artifacts will be required.

- A mouse.

- MS-DOS or PC-DOS version 3.1 or later.

- Windows version 3.1 in enhanced mode.

\subsection{Running the Setup}

Follow the steps belong to install the Reuse with Facets tool on your workstation. Do not copy the files directly onto your system, since they are in the compressed mode.

1. Insert Disk \#1 in drive A.

2. From the File menu of the Program Manager or File Manager, choose Run.

3. Type a:setup

4. Follow the Setup instructions on the screen. 
During setup, you will be asked to provide the path of the directory where you want to install the files related to the tool. This setup program will install all the files, including the database files, in this directory. If multiple users are going to use the tool, move the database-related files, i.e., all files with extensions mdb and $\mathrm{ldb}$, to a central location. Then change the default data source entry in the initialization file (rwf.ini) to reflect this change.

When multiple users are going to use the tool, the setup program must be run on each of these workstations. Since all these users are going to use the database at the central location, the database files created on the users' workstations must be deleted. You may also use several different databases (for different domains) and change the initialization file entry to correspond to this.

\section{Insertion of Software Artifacts into the System}

When the tool is started, the user is forced to login. This is, however, used not to enforce security but to obtain the name of the user. This information is used later in compiling the usage reports. A picture of the Login box is provided in Figure E-1.

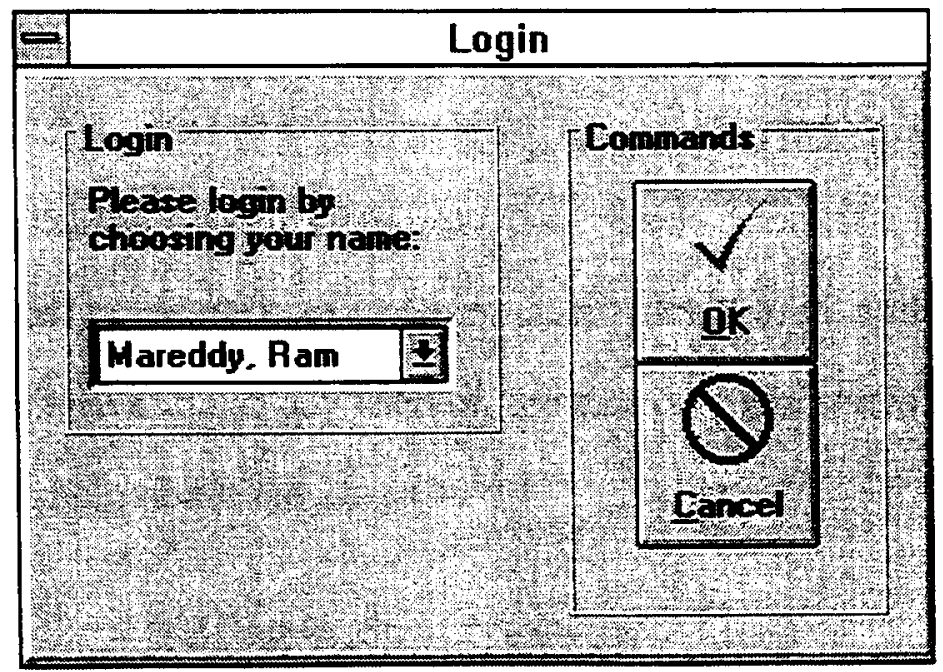

Figure E-1. Login box 


\subsection{Main Window}

All the functionality of the Reuse with Facets can be accessed through the main window with the help of menus and tool buttons provided there. Figure E-2 is a representation of this window.

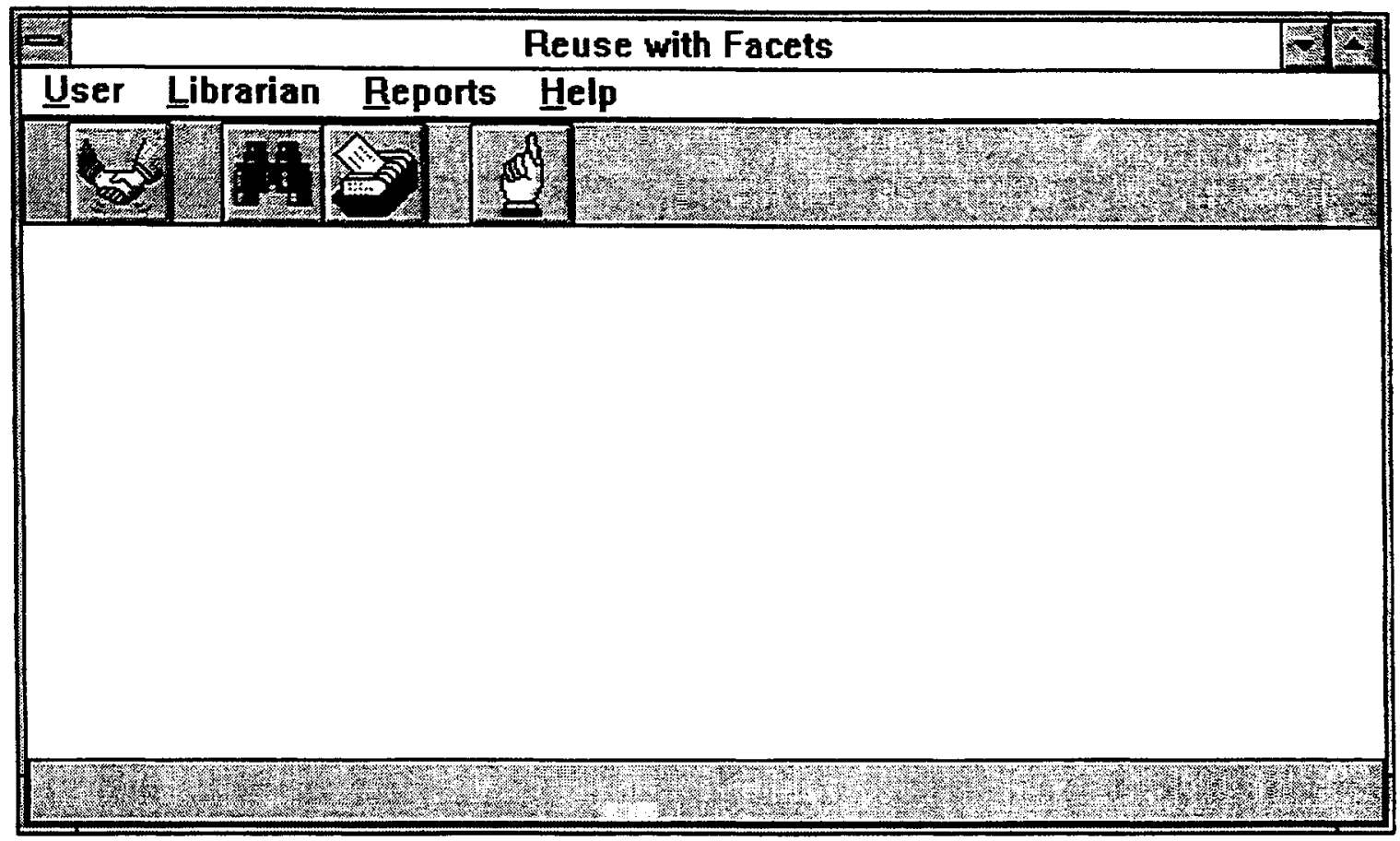

Figure E-2. Main Window of Reuse with Facets

The menus attached to this window, i.e., User, Librarian, Reports, and Help, provide the functionality of this tool. Commands like search and insert can also be accessed through the tool buttons provided on the tool bar. The status bar at the bottom of the window provides help about the items.

\subsection{Preparation of Software Artifact}

When a Developer or the Librarian comes across a piece of software artifact that is potentially reusable in a later software development, the Developer/Librarian should 
first prepare the artifact to insert into the system. A software artifact need not necessarily be a code fragment, it can be a specification, a documentation, a design structure, a module-level implementation structure, or any other software component. This artifact must be saved (after any appropriate separation or modification) in a file. Then choose the Insert command from the User menu of the tool. The dialog box shown in Figure E-3 will be presented to the user.

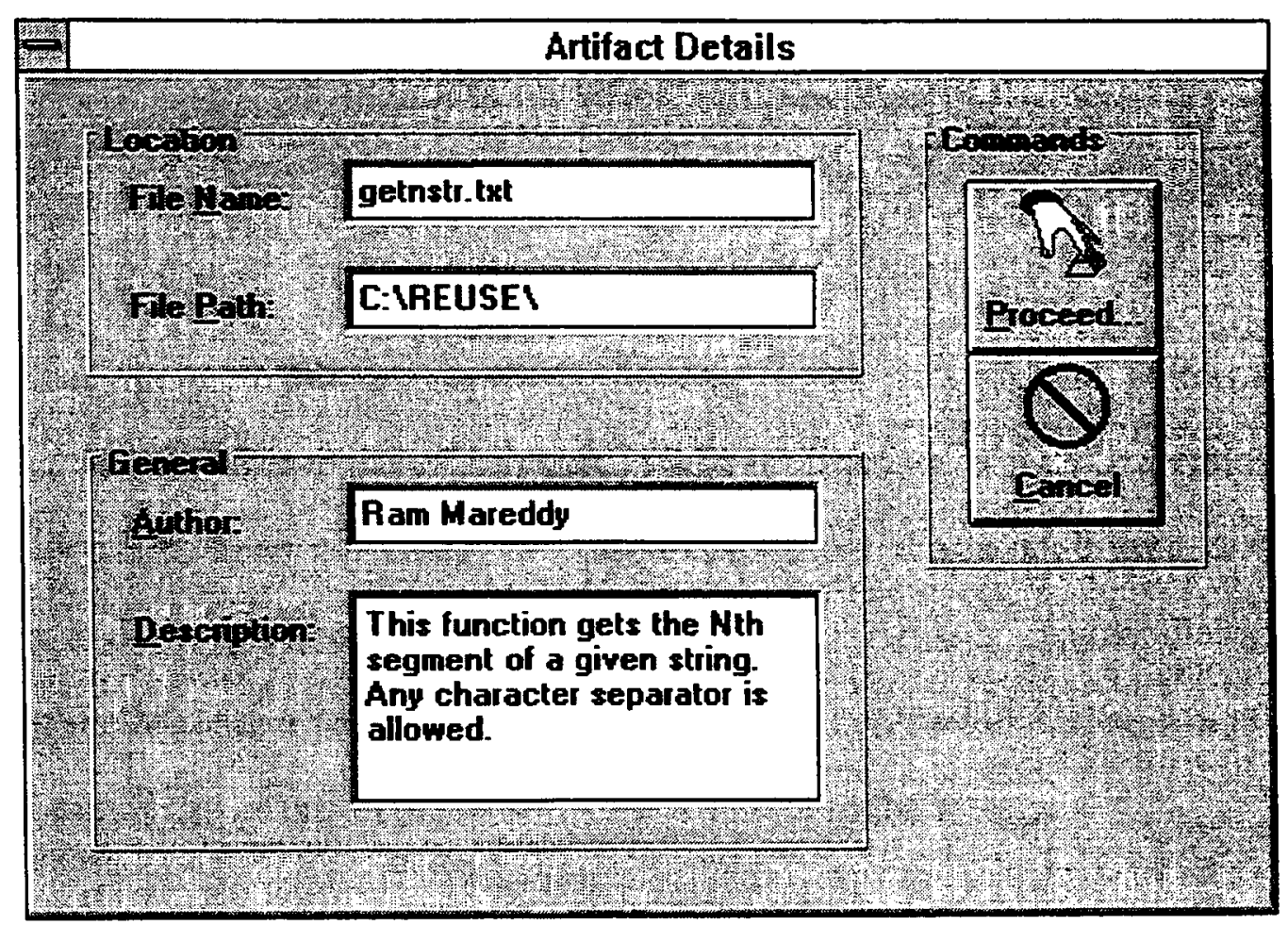

Figure E-3. Artifact Details

In this dialog box (Figure E-3) the location and other general details are requested from the user. Location details, i.e., the name of the file in which the software artifact under consideration is saved and the path to reach that file, must be inputted by the user. The tool will check these details to make sure that the file exists at the aforementioned 
place. Entry of the general details, i.e., the author of the artifact and a description, is not mandatory, but highly recommended.

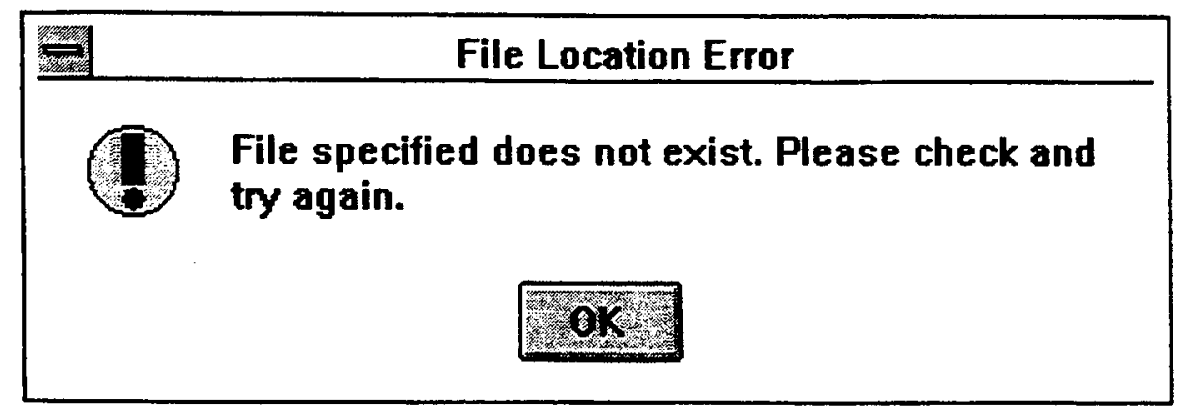

Figure E-4. Artifact Existence Error

After these details are entered, the user needs to click the button captioned Proceed..., which will take the user to the classification screen. If the file mentioned in the above dialog box does not exist, an error message (as shown in Figure E-4) will be displayed and the processing will come back to the Artifact Details dialog box for correcting the name and path of the file. The cancel button on this dialog box provides an option to cancel the insertion of the software artifact into the system.

\subsection{Classification of Software Artifact}

If the software artifact mentioned in the previous dialog box does exist in the system, the next step is to classify that artifact according to the facets and the associated vocabulary. For this purpose, a dialog box similar to Figure E -5 will be presented to the user. 


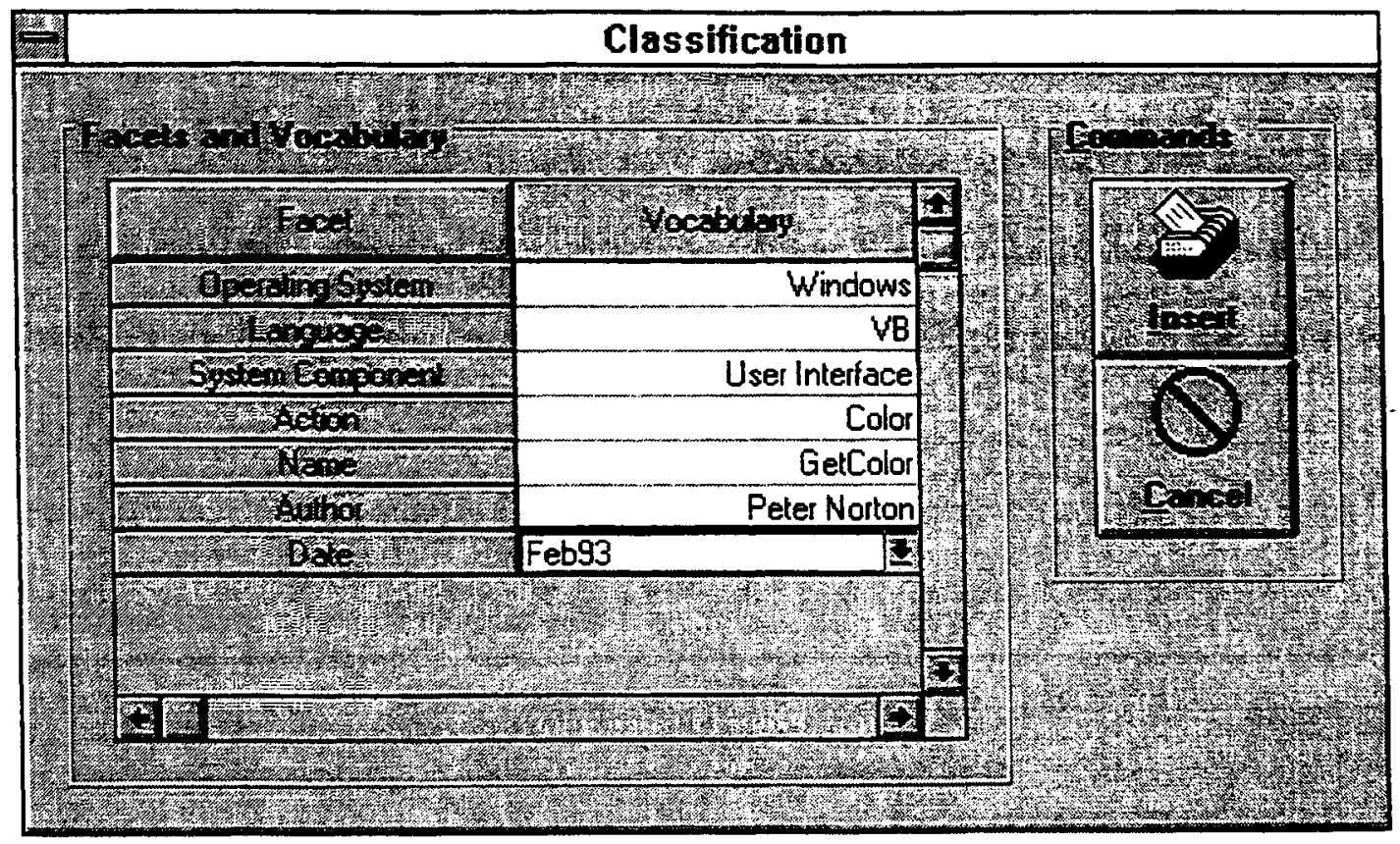

Figure E-5. Classification of the Artifacts

The first column in Figure E-5 contains the list of facets with which this software artifact can be described. For each of these facets there is another list of vocabulary in the second column. Click on the pull-down button on these list boxes to choose the appropriate word to describe that particular facet. Once the selection of vocabulary is completed, click on the Insert button to insert this artifact into the system with the classification described on the table in Figure E-5.

\section{Retrieval of Software Artifacts from the System}

This section is of particular interest to Developer/Reuser rather than the Librarian. Hence, this section can be treated as the Reuser's guide and rest of this guide can be taken as the Librarian's Guide. The process of obtaining an artifact from the repository contains two steps, search and retrieval. 


\subsection{Search of the Repository}

Figure E-6 depicts the dialog box presented to the user for the purpose of searching for software artifacts which match a given specification. This functionality can be obtained by choosing the command Retrieval from the User menu in the main window of the tool.

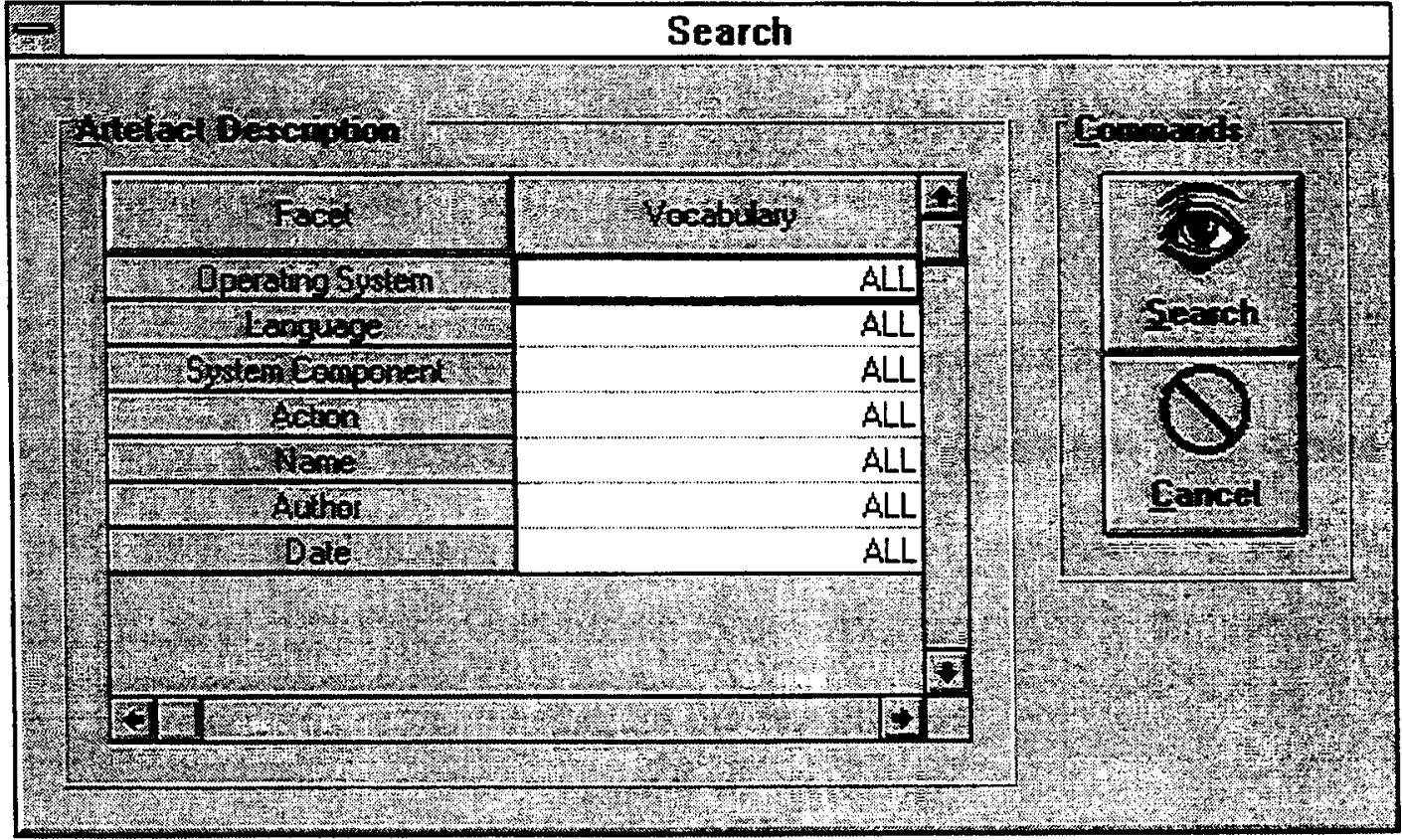

Figure E-6. Search of the Artifacts

Search the Repository
There are 3 artifacts present in the system with your
classification. Do you want to view them?
Yos No

Figure E-7. Hits in the Search of Repository 
This dialog box (Figure E-6) is similar to the classification dialog box. The first column contains the list of all facets available to describe an artifact. The second column contains the vocabulary for each artifact. After the selection of vocabulary is completed, click on the Search button to search for the artifact with given class.

The search of the repository can result in a hit of one more artifacts, or there can be a miss, i.e., no artifact available in the system matching the description given by the user. If there are hits as shown in Figure E-7, the tool will present a dialog box similar to the one in Figure E-10 for the retrieval of the artifact, otherwise (if there are no hits, as shown in Figure E-8) a dialog box to use synonyms as in Figure E-9 will be presented.

\subsection{Synonyms}

If the user chooses to search the system with synonyms, an extra column will be added to the search dialog box. This column provides the flexibility to the user to use the synonyms for an individual facet rather than for all facets. Figure E-9, which is similar to the search dialog box, has an extra column to indicate Yes or No to the use of synonyms for the corresponding facet and vocabulary in the columns.

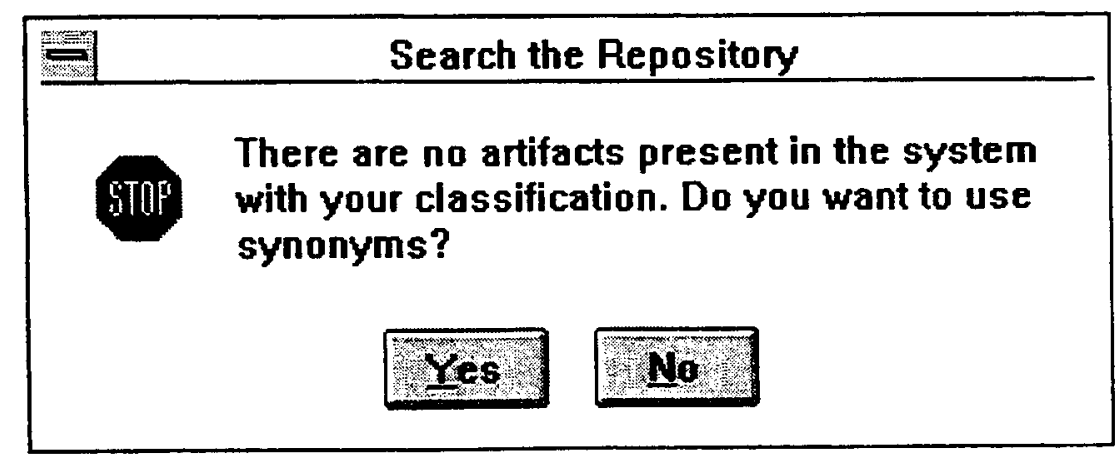

Figure E-8. Suggestion for synonyms 
More Yes's in the synonyms column (in Figure E-9) will take more time to search the repository for artifacts. This will also broaden the search criteria and there will be more chance of a hit for an artifact. After the search, if there are any hits in the system, they will be presented in the Retrieval dialog box (Figure E-10) to retrieve the artifact from the system, else the synonyms dialog box will be presented to further broaden the scope of search.

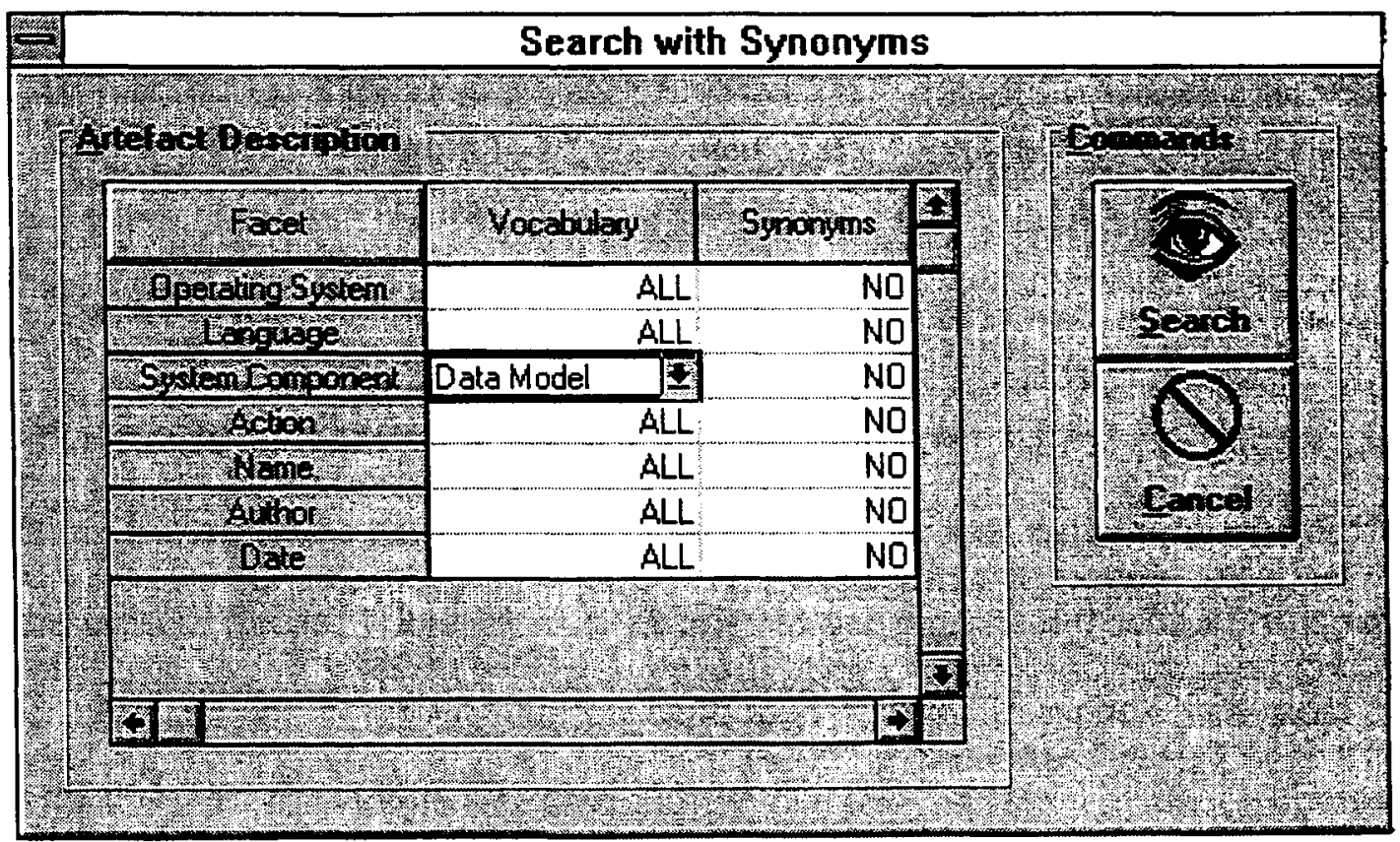

Figure E-9. Search with Synonyms

\subsection{Retrieval of the Artifacts}

If any hits result from the search for software artifacts, they will be presented in the retrieval dialog box with all the physical characteristics. 


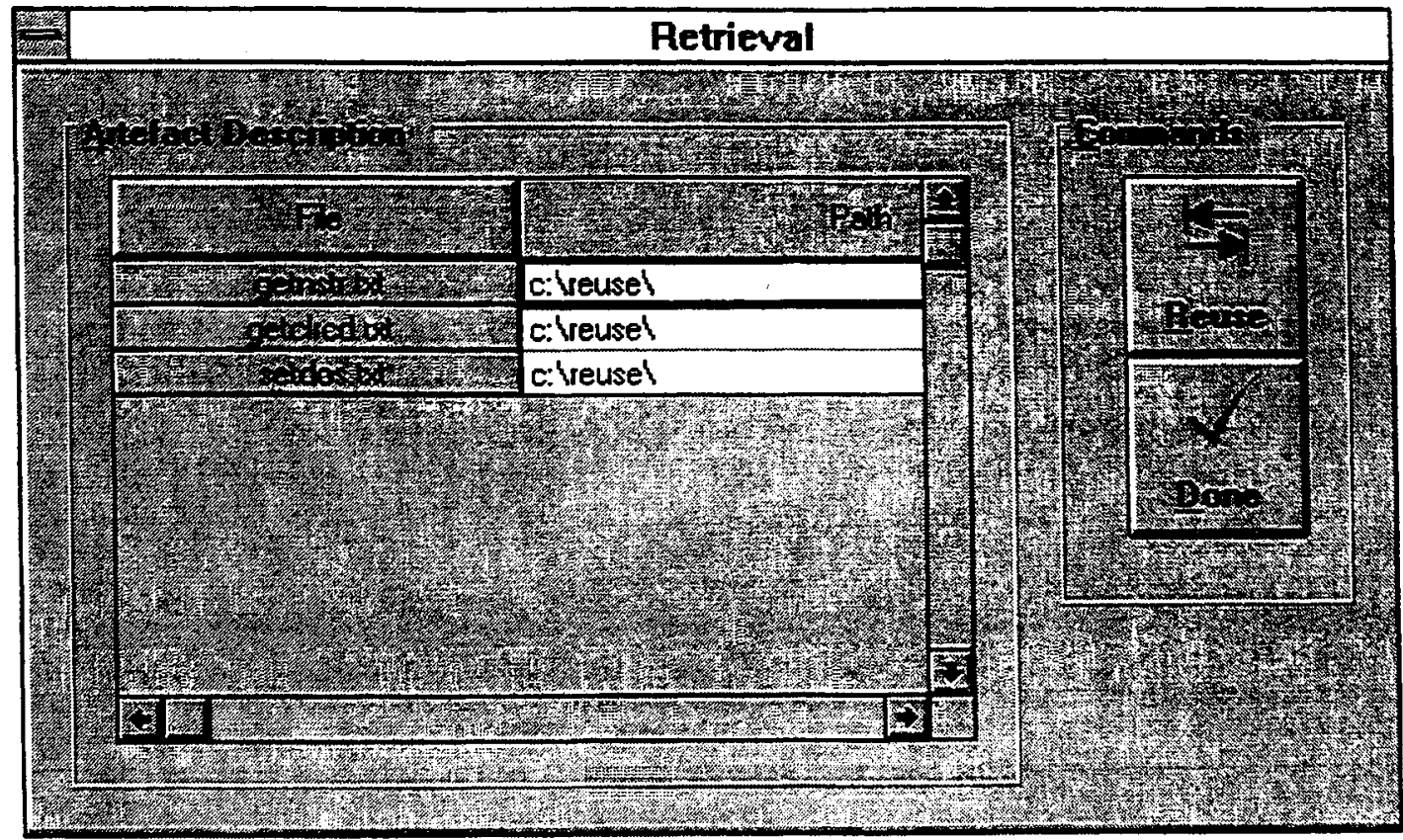

Figure E-10. Retrieval of the Artifacts

The Retrieval dialog box presents the filename, path of the file, author, and date of insertion. By using the details in this screen, the reuser then can choose the artifact that meets the present needs. When the user decides to use a particular artifact, it is requested that user click on the command button captioned Reuse. This click will store it as an event with the currently-logged-in user and the currently-selected artifact as participants. This information will be used in the generation of the performance reports.

\section{System Administration}

System administration is a Librarian-specific function. All the necessary commands are available from the Librarian menu in the main window. These commands provide the ability to the Librarian to add or delete facets, add or delete a vocabulary item, delete the artifact entry in the database, and add or delete the entries in the thesaurus. A second set of commands is provided to administer the user-related issues. These commands provide the ability to the Librarian to add or delete users, and add or delete roles. 


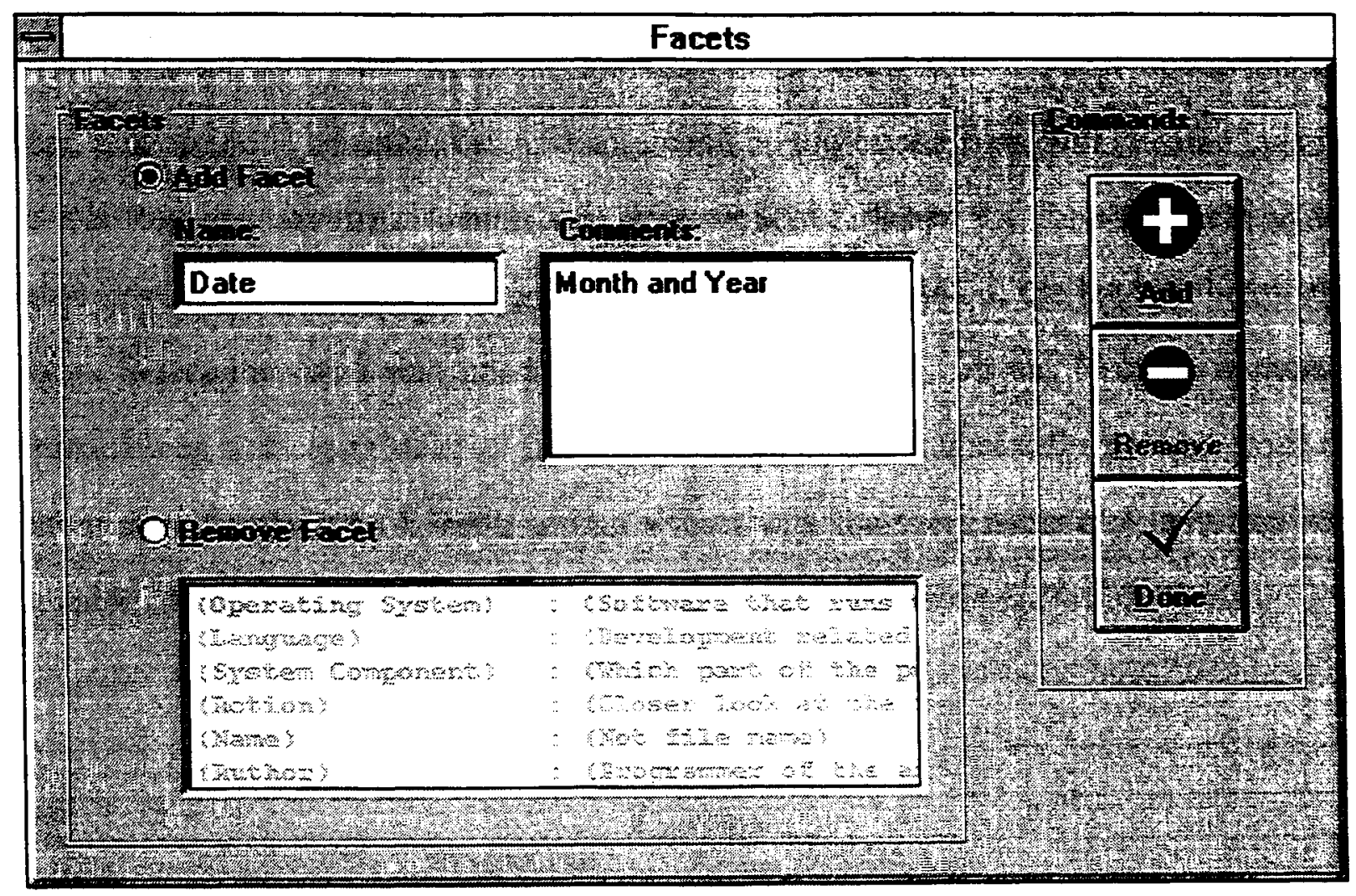

Figure E-11. Administration of Facets

\subsection{Facets}

To add or remove facets, choose the Facet command from the Librarian menu. A dialog box similar to Figure E-11 will be presented to facilitate the administration of facets.

To add a facet to the system, select the Add Facet option button. When this option is selected, the Add command button on the right will become active and the Remove command button will become inactive, i.e., it will be grayed out. With the Add Facet option button selected, input the name of the facet and a brief description in the two text boxes provided below the option button. Then, to add this facet to the system, click on the Add button. 
To remove a facet from the system, choose the Remove Facet option button. When this option is selected, the Add command button on the right will become inactive and the Remove command button will become active. When the Remove facet option button is selected, a list of the existing facets will be presented in the list box below this option. Select the facet that needs to be deleted and click on the Remove command button. When a facet is deleted from the system, it is automatically deleted from the artifact classification. As a result adding a facet with the same name at a later time will not automatically restore the earlier classification for the existing artifacts.

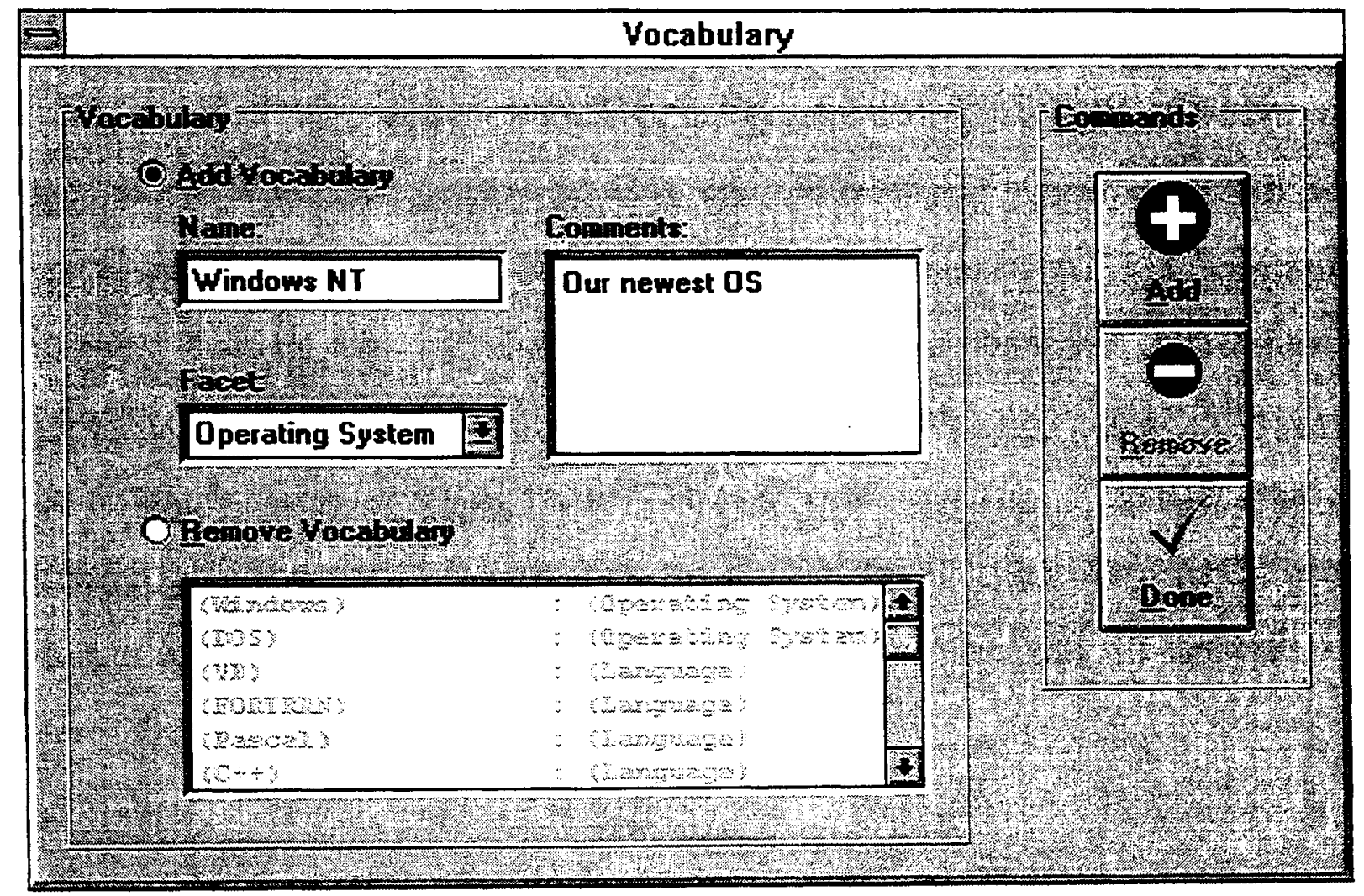

Figure E-12. Administration of Vocabulary 


\subsection{Vocabulary}

To add or remove a vocabulary item, choose the Vocabulary command from the Librarian menu. A dialog box similar to Figure E-12 will be presented to facilitate the administration of the vocabulary.

To add a vocabulary term to the system, select the Add Vocabulary option button. When this option is selected, the Add command button on the right will become active and the Remove command button will become inactive, i.e., it will be grayed out. With the Add Vocabulary command button selected, input the name of the vocabulary term, choose the facet that this term will describe, and a brief description in the two text boxes, and a selection box presented below. Then to add this vocabulary term to the system, click on the Add button.

To remove a vocabulary term from the system, choose the Remove Vocabulary option button. When this option is selected, the Add command button on the right will become inactive and the Remove command button will become active. When the Remove Vocabulary button is selected, a list of the existing vocabulary terms will be presented in the list box below this option. Select the vocabulary term that needs to be deleted and click on the Remove command button. When a vocabulary term is deleted from the system, it is automatically deleted from the artifact classification. As a result, hence adding a vocabulary term with the same name at a later time will not automatically restore the earlier classification for the existing artifacts.

\subsection{Artifact}

To remove an artifact from the classification, choose the Artifact command form the Librarian menu (to insert an artifact into the system, use the Insert command from the User menu). A dialog box similar to Figure E-13 will be presented to the user to facilitate the administration of the Artifacts. 


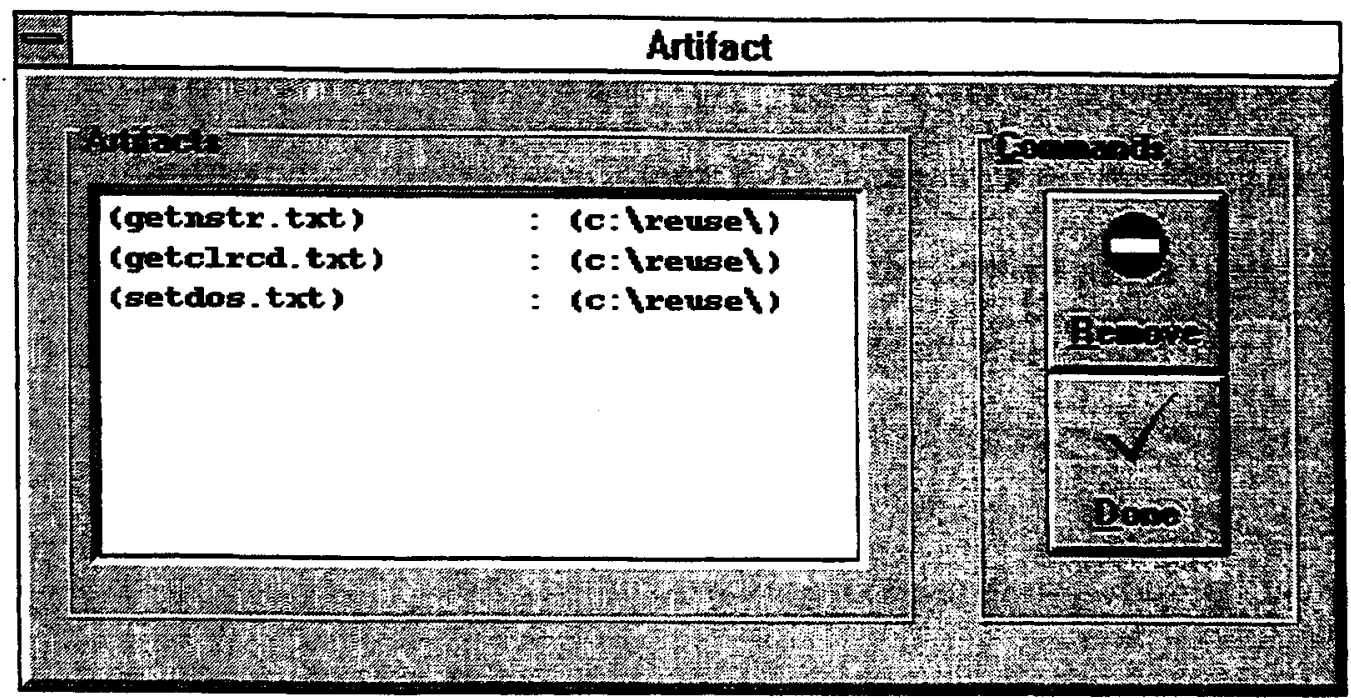

Figure E-13. Administration of Artifacts

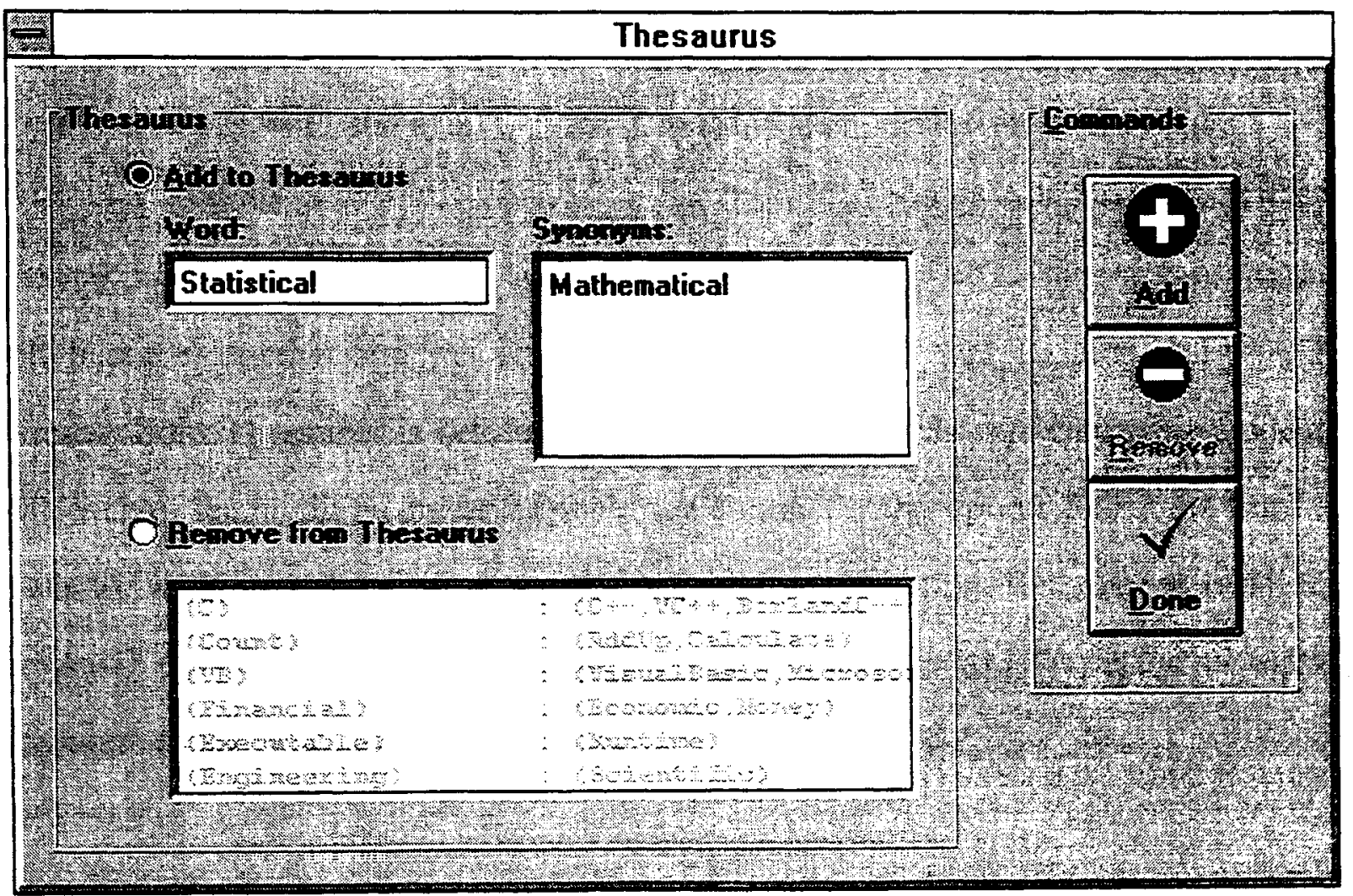

Figure E-14. Administration of Thesaurus 
To remove an artifact from the system, first select the artifact from the list box in the Artifact Administration dialog box (Figure E-1). Then click on the Remove command button on the right. This will only remove the entry of the artifact in the tables of the tool, i.e., it will not delete the physical file from the hard disk.

\subsection{Thesaurus}

To add or remove a word-synonym string combination, choose the Thesaurus command from the Librarian menu. A dialog box similar to the Figure E-14 will be presented to the user to facilitate the administration of thesaurus items.

To add a Word-Synonym String to the system, select the Add to Thesaurus option button. When this option is selected, the Add command button on the right will become active and the Remove command button will become inactive, i.e., it will be grayed out. With the Add to Thesaurus option button selected, input the word and the string of synonyms, separated by commas, in the two text boxes provided below the option button. Then, to add this word and synonym string to the system, click on the Add button.

To remove a Word-Synonym string from the system, choose Remove from Thesaurus option button. When this option is selected, the Add command on the right will become inactive and the Remove command button will become active. When Remove from Thesaurus is selected, a list of existing words and synonyms associated with each of them will be presented in the list box below this option. Select the word that needs to be deleted and click on the Remove command button. Removal or addition of terms will not result in any changes to the classification of the artifacts, as the thesaurus is a separate entity from rest of the database.

\subsection{User}

To add a user to the system with all the accompanying information, or remove a user from the system, choose the User command from the Librarian menu. A dialog box 
similar to Figure E-15 will be presented to the Librarian to facilitate the administration of users.

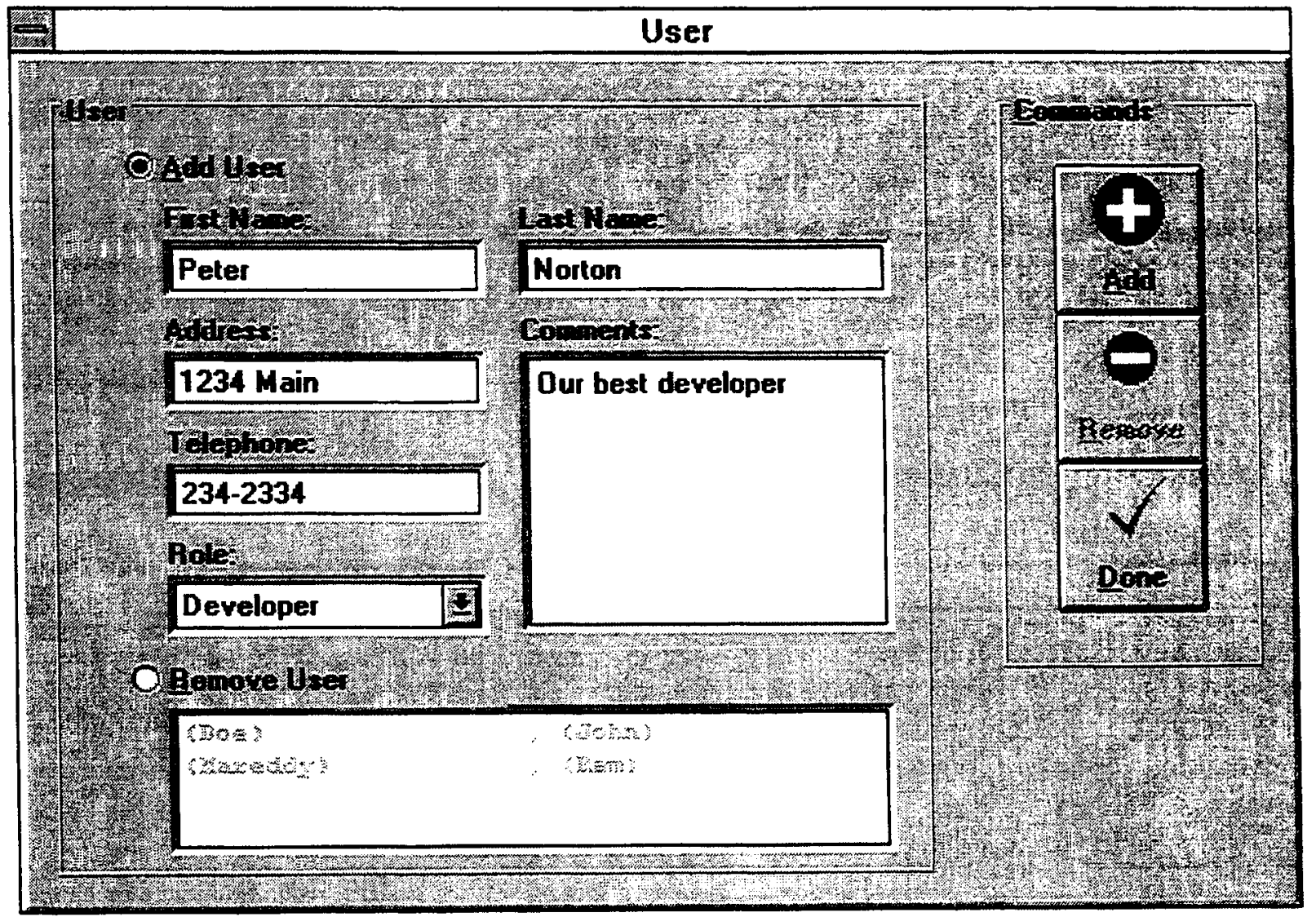

Figure E-15. Administration of Users

To add a user to the system, select the Add User option button. When this option is selected, the Add command button on the right will become active and the Remove command button will become inactive, i.e., it will be grayed out. With the Add User option selected, input the first name, last name, address, telephone, role, and comments in the text boxes and the selection box provided below the option button. Then, to add this user to the system, click on the Add button. 
To remove a user from the system, choose the Remove User option button. When this option is selected, the Add command on the right will become inactive and the Remove command button will become active. When the Remove User option button is selected, a list of existing users will be presented in the list box below this option. Select the user that needs to be deleted and click on the Remove command button.

\subsection{Role}

To add or remove a role, choose the Role command from the Librarian menu. A dialog box similar to Figure E-16 will be presented to the user to facilitate the administration of roles.

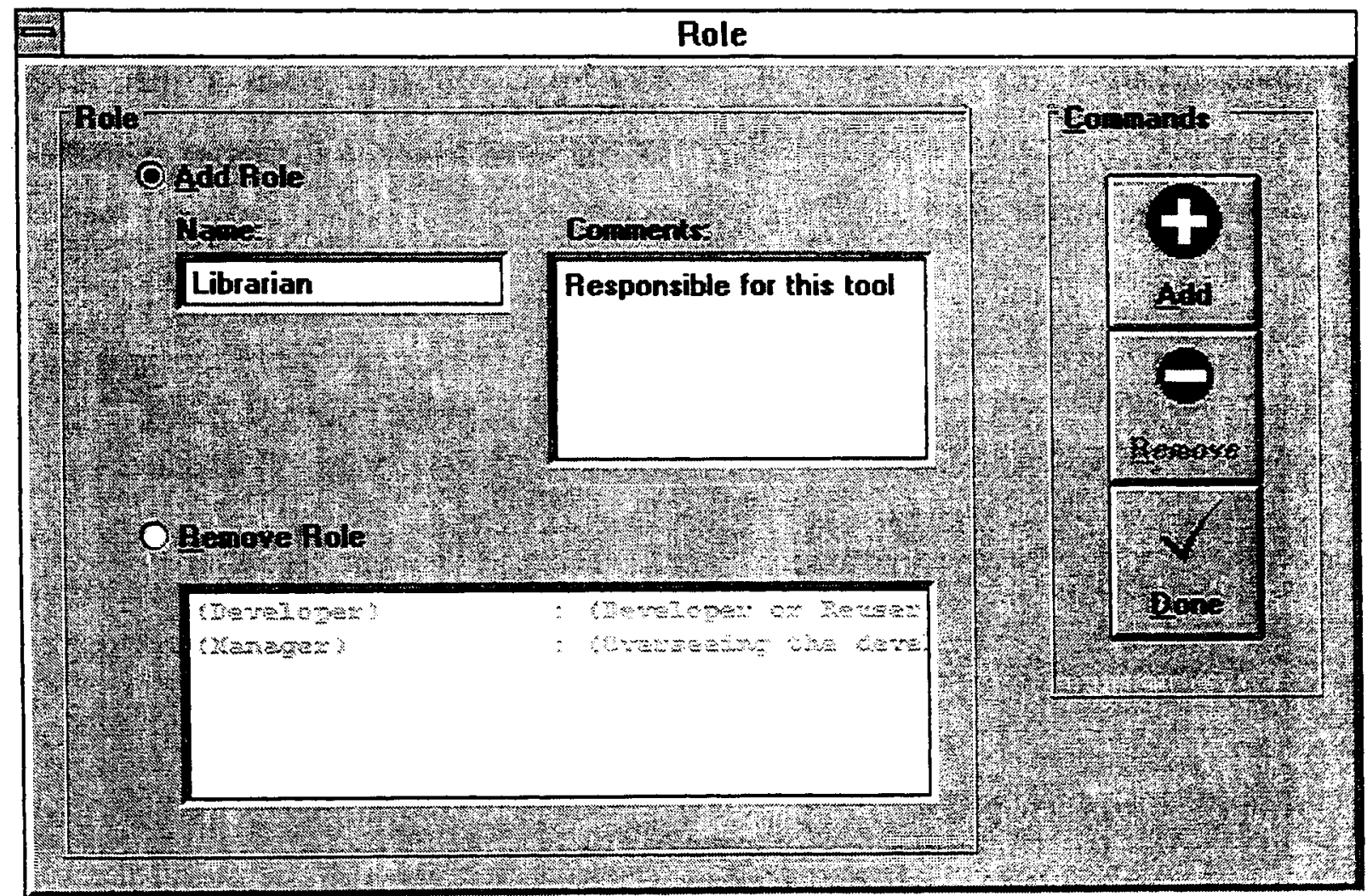

Figure E-16. Administration of Roles 
To add a role to the system, select the Add Role option button. When this option is selected, the Add command button on the right will become active and the Remove command button will become inactive, i.e., it will be grayed out. With the Add Role option button selected, input the name of the role and a brief description in the two text boxes provided below the option button. Then, to add this role to the system, click on the Add button.

To remove a role from the system, choose the Remove Role option button. When this option is selected, the Add command button on the right will become inactive and the Remove command button will become active. When the Remove Role option button is selected, a list of the existing roles will be presented in the list box below this option. Select the role that needs to be deleted and click on the Remove command button. Please note here that the role of Librarian cannot be deleted, since someone has to maintain this system. All the users with the deleted role will be temporarily given the Librarian role. These roles should be changed later on.

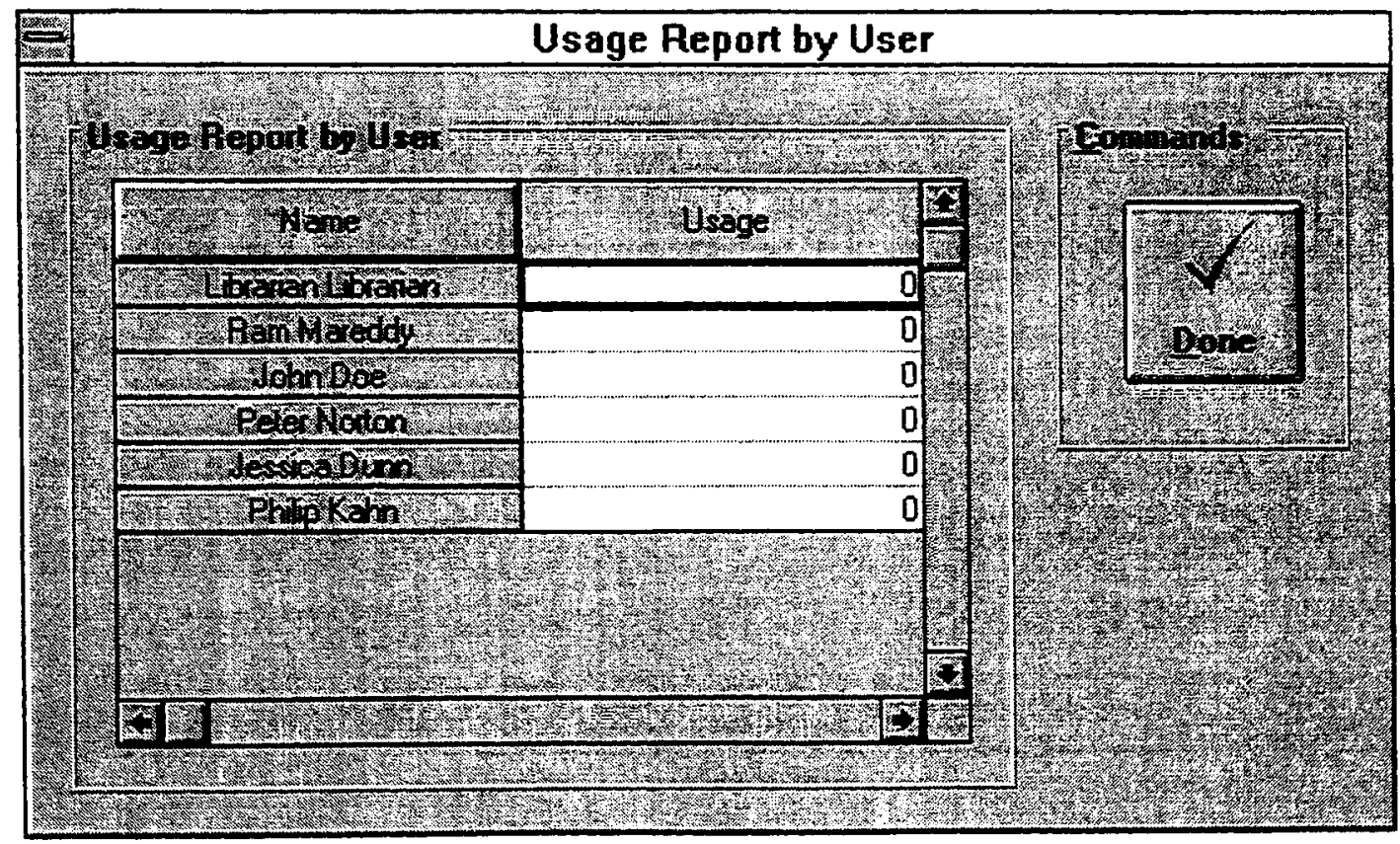

Figure E-17. Usage Report by User 


\section{Report Generation}

Two types of reports can be generated from this tool. The first type deals with the amount of reuse done per user, and the second type deals with amount of reuse done by the artifact.

\subsection{Usage Report By User}

To obtain the usage report per user, choose the command User Report from the Reports menu. This will generate a report that looks like Figure E-17.

This report gives information about how many functions a user has reused. This information can be used to reward that particular user, or for other organizational purposes.

\subsection{Usage Report by Artifact}

To generate this report, choose the command Artifact Report from the Reports menu. This will generate a report that looks like Figure E-18.

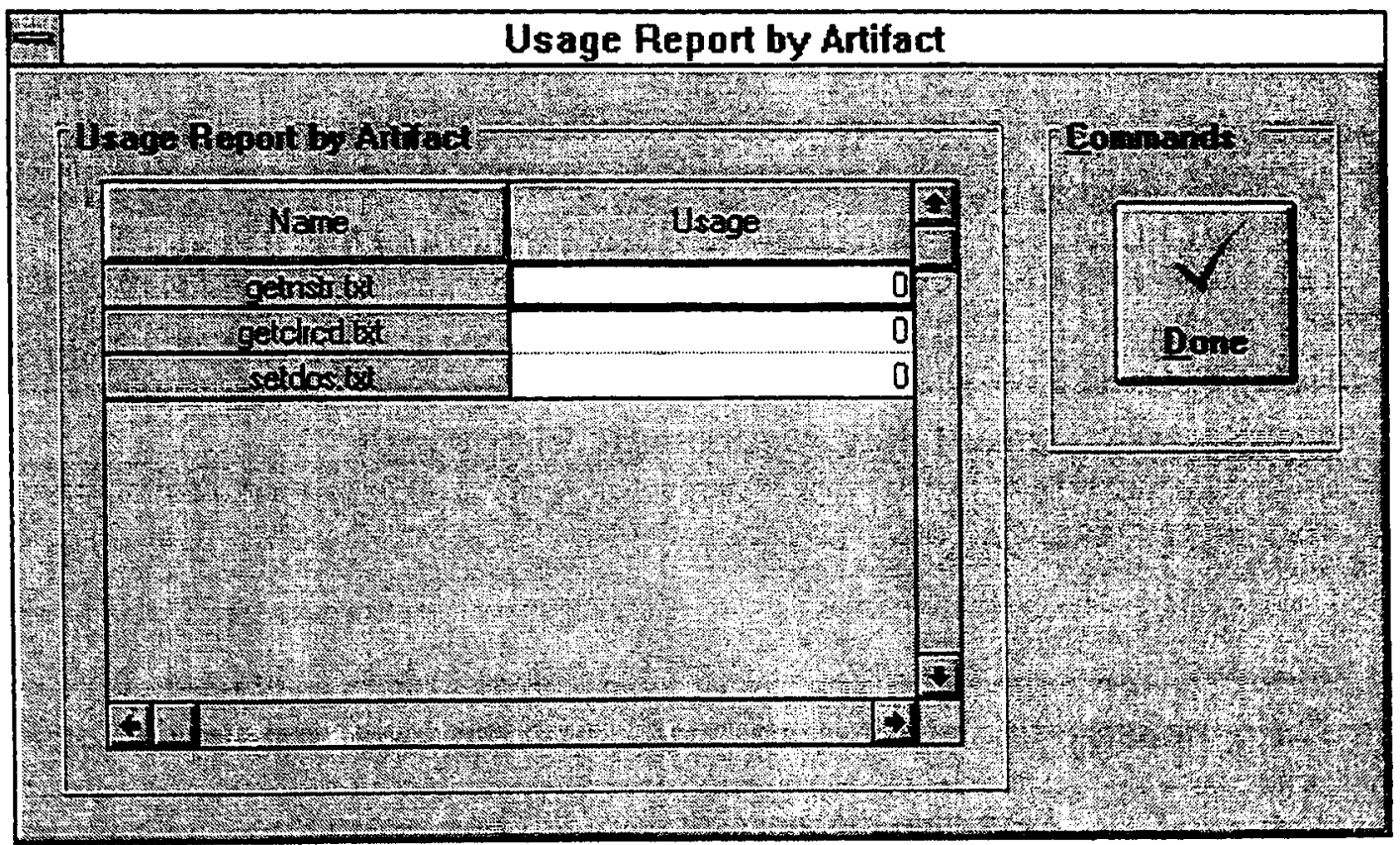

Figure E-18. Usage Report By Artifact 
This report lists all the software artifacts listed in the system and the number of times each artifact is reused. This number will help the Librarian in making the decision about removing artifacts due to non-use or low reuse.

\section{Help}

In the Help menu, an "About..." command is also provided, which gives the version details of the Reuse with Facets tool. This dialog box is depicted in Figure E-19.

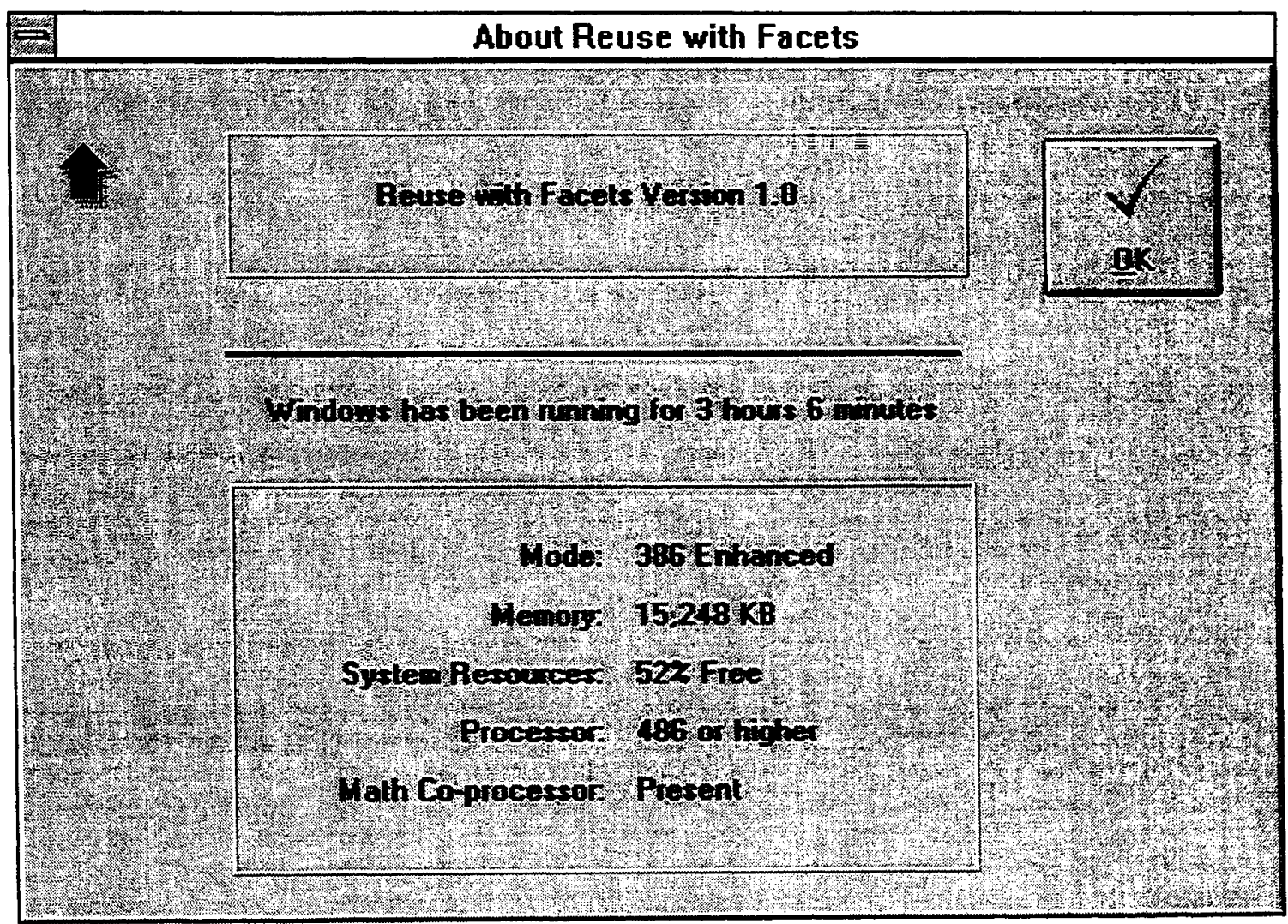

Figure E-19. About Reuse with Facets

The About box also provides the system-related information concerning the processor, math co-processor, and the available system resources. 


\section{APPENDIX F}

PROGRAM LISTINGS 
Make File For The Project

RWFGLOB . BAS

C: \WINDOWS \SYSTEM \SPIN . VBX

$C: \backslash W I N D O W S \backslash S Y S T E M \backslash T H R E E D$.VBX

C: $\backslash W I N D O W S \backslash S Y S T E M \backslash S P R E A D 20$. VBX

RWEABOUT. FRM

RWFUSAGE. FRM

RWFROLE . FRM

RWFUSER . FRM

RWFTHES . FRM

RWFVOCAB . FRM

RWFRTRV.FRM

RWFCLASS. ERM

RWEMAIN . FRM

RWEFACET . FRM

RWEDET. FRM

RWFSRCH. FRM

RWFARDEL . FRM

RWFCLREP . FRM

RWELOGIN. FRM

Projwinsize $=128,386,248,310$

Projwinshow $=2$

IconForm= " frmMain"

Title= "RWF"

ExeName $=$ "RWF . EXE"

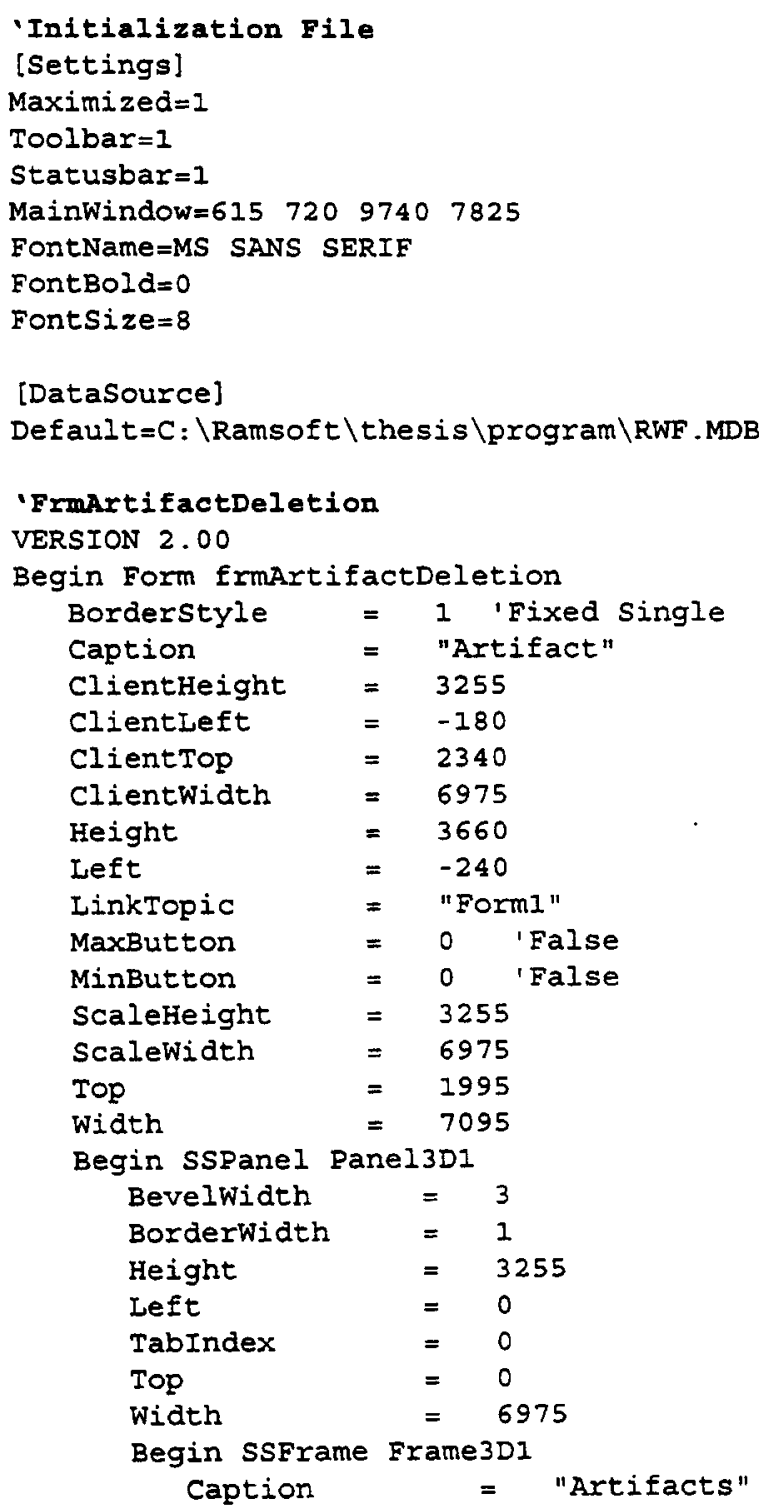




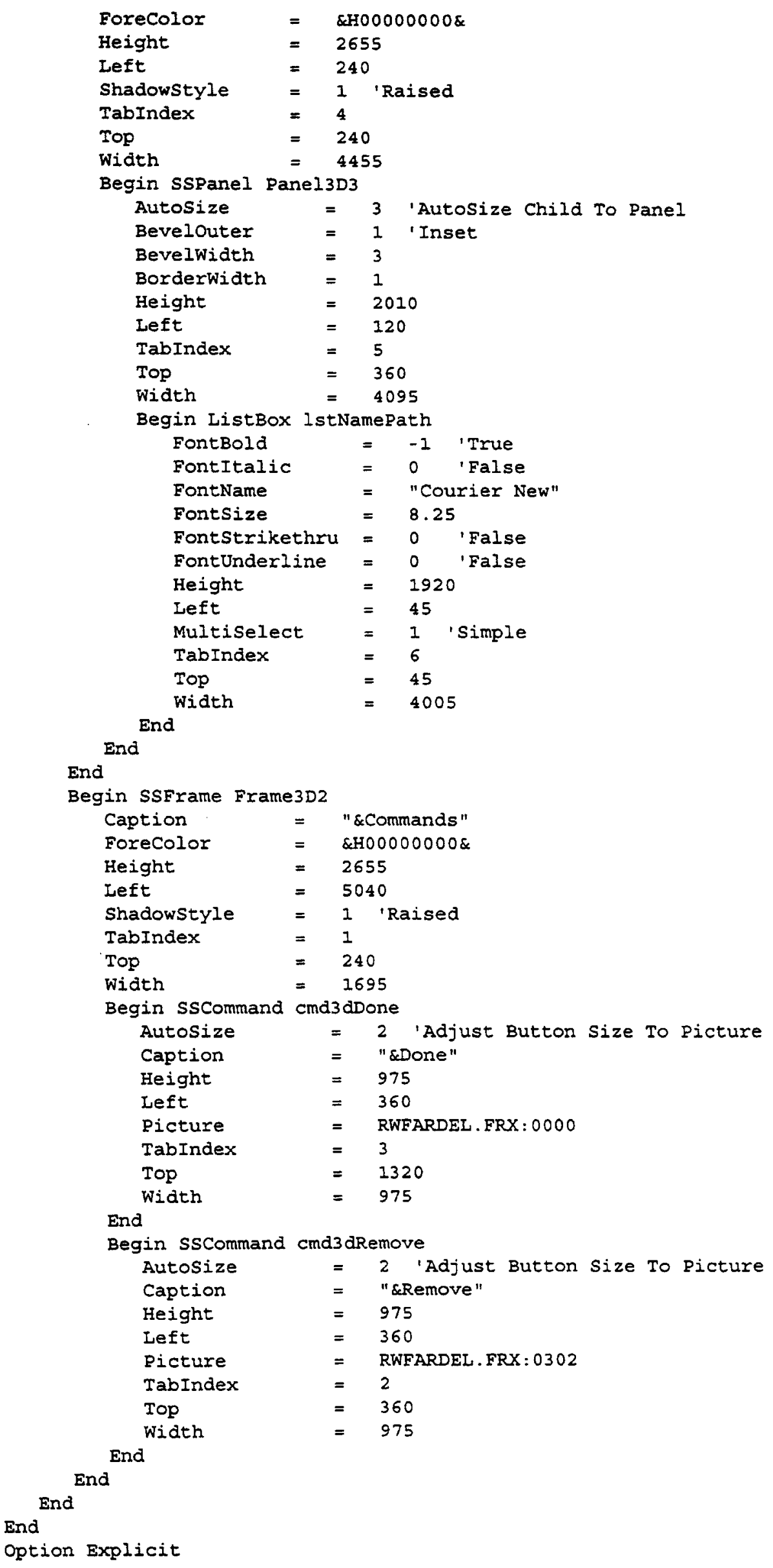


Sub cma3dDone_click ()

Unload Me

End Sub

Sub cmd3dRemove_Click ()

Removeartifact

End Sub

'Name: FillBoxes

'Type: subroutine

'Parameters: None

Returns: None

'Description: This subroutine fills all the box, i.e. listbox with information.

'This can also be called after REMOVE is done.

'Author: Ram Mareddy

' '

Sub FillBoxes ()

Dim sqryName As String

Dim sNamePath As String

Dim dsName As Dynaset

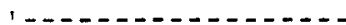

'Get Name and Group

1 .

sqryName = "SELECT ArtifactFileName, ArtifactPath FROM ARTIFACT"

Set dsName $=$ gdbRwf. CreateDynaset (sqryName)

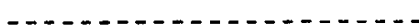

'Fill Name+Path List Box

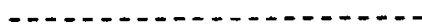

'First clear the list box.

istNamePath. Clear

Do Until dsName.EOF

sNamePath = "(" + dsName ("ArtifactFileName") + ")"

sNamePath $=$ sNamePath + Space $\$(20$ - Len $($ sNamePath $))+"$ : "

sNamePath = sNamePath + "(" + dsName ("ArtifactPath") + ")"

IstNamePath. AddItem sNamePath

dsName MoveNext

Loop

End Sub

Sub Form_Load ()

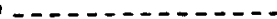
' Center the form
1................
CenterForm Me

ind

' Fill boxes on the form with details 
FillBoxes

End Sub

'Name: RemoveArtifact
'Type: Subroutine
' Parameters: None
' Returns: None
'Description: This function removes one or more artifacts selected in the
'remove list box.
''Author: Ram Mareddy
'-

Sub Removeartifact ()

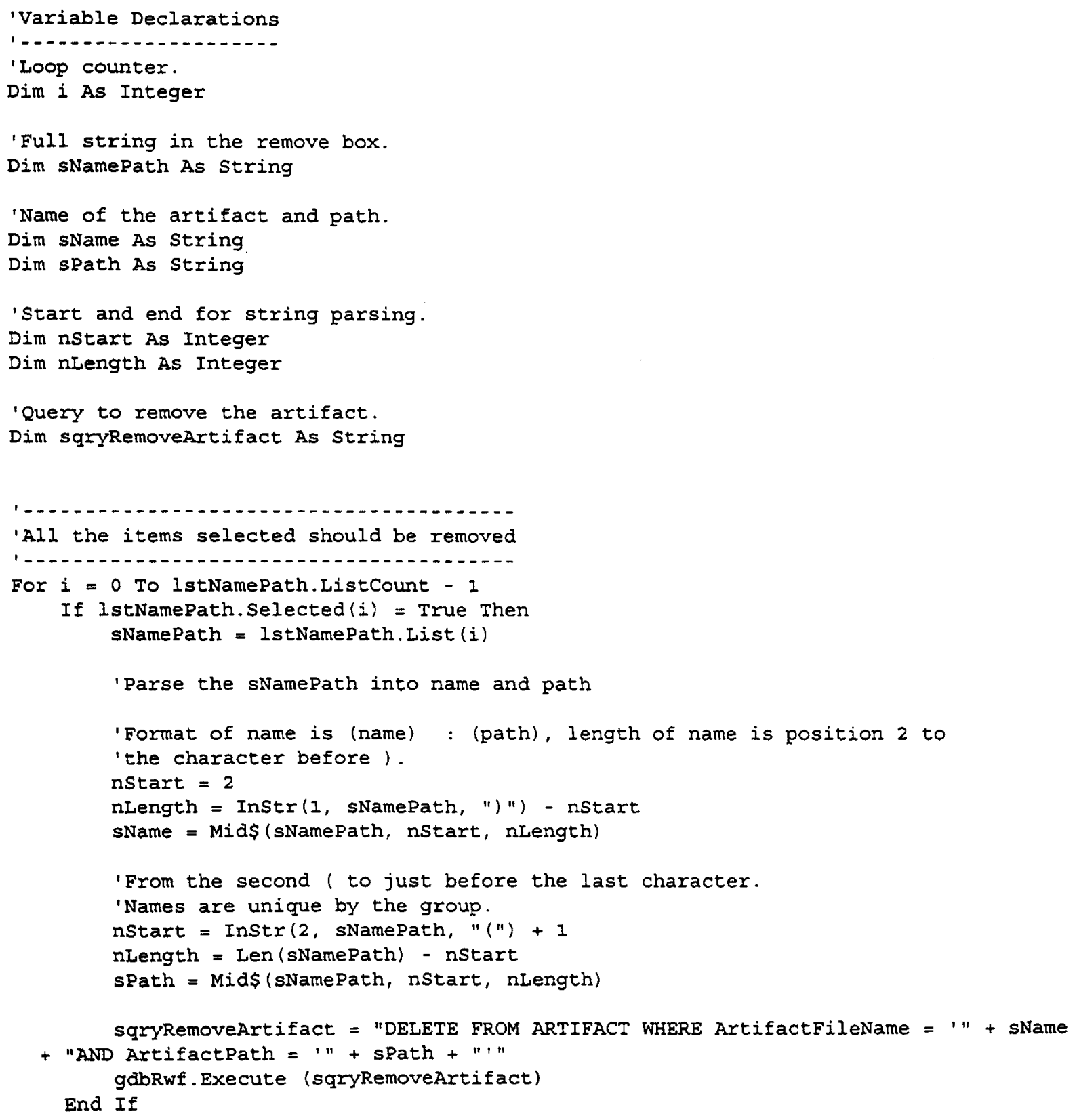


Next

' Refresh boxes again

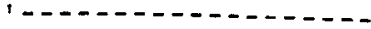

FillBoxes

End Sub

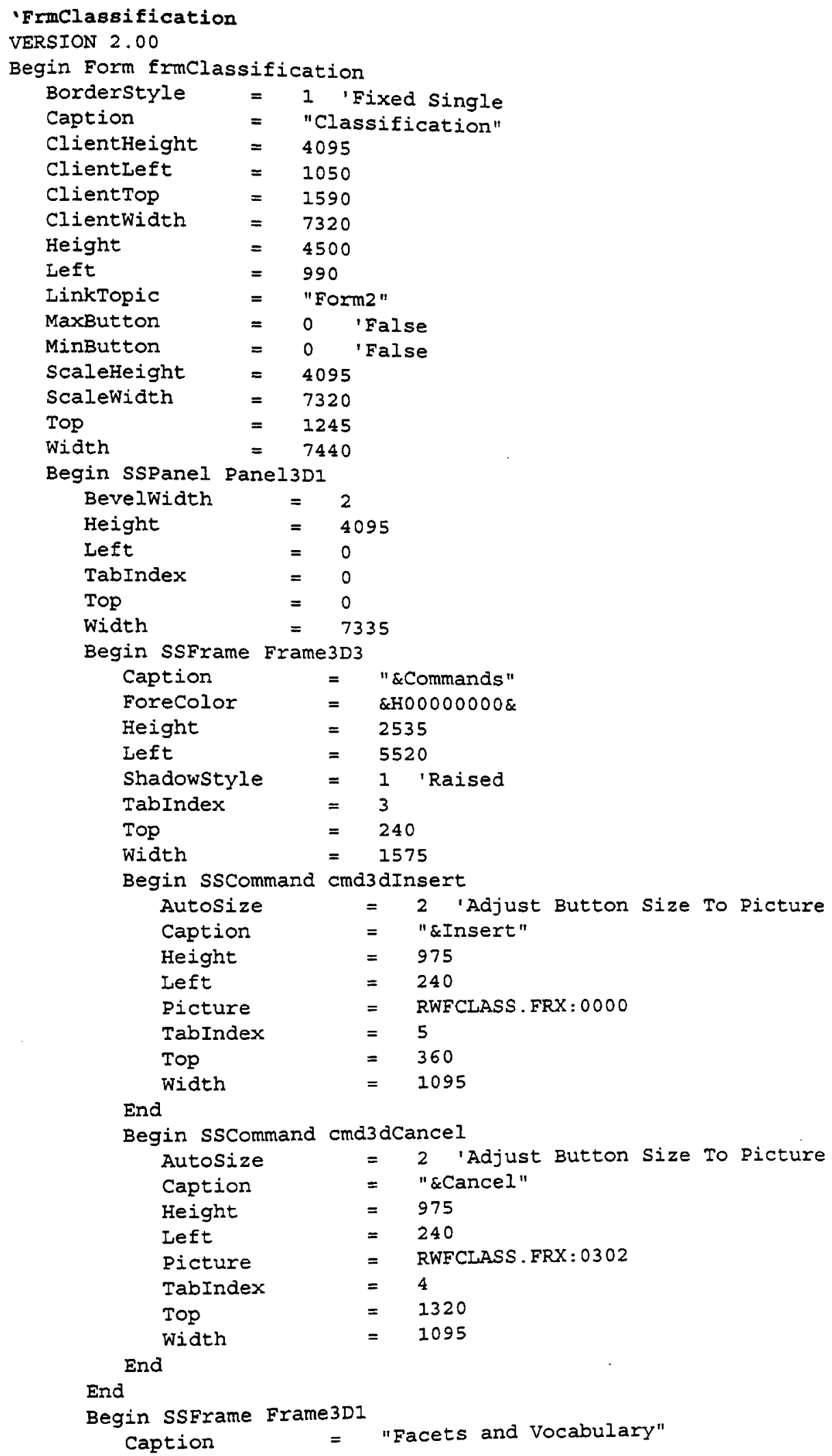




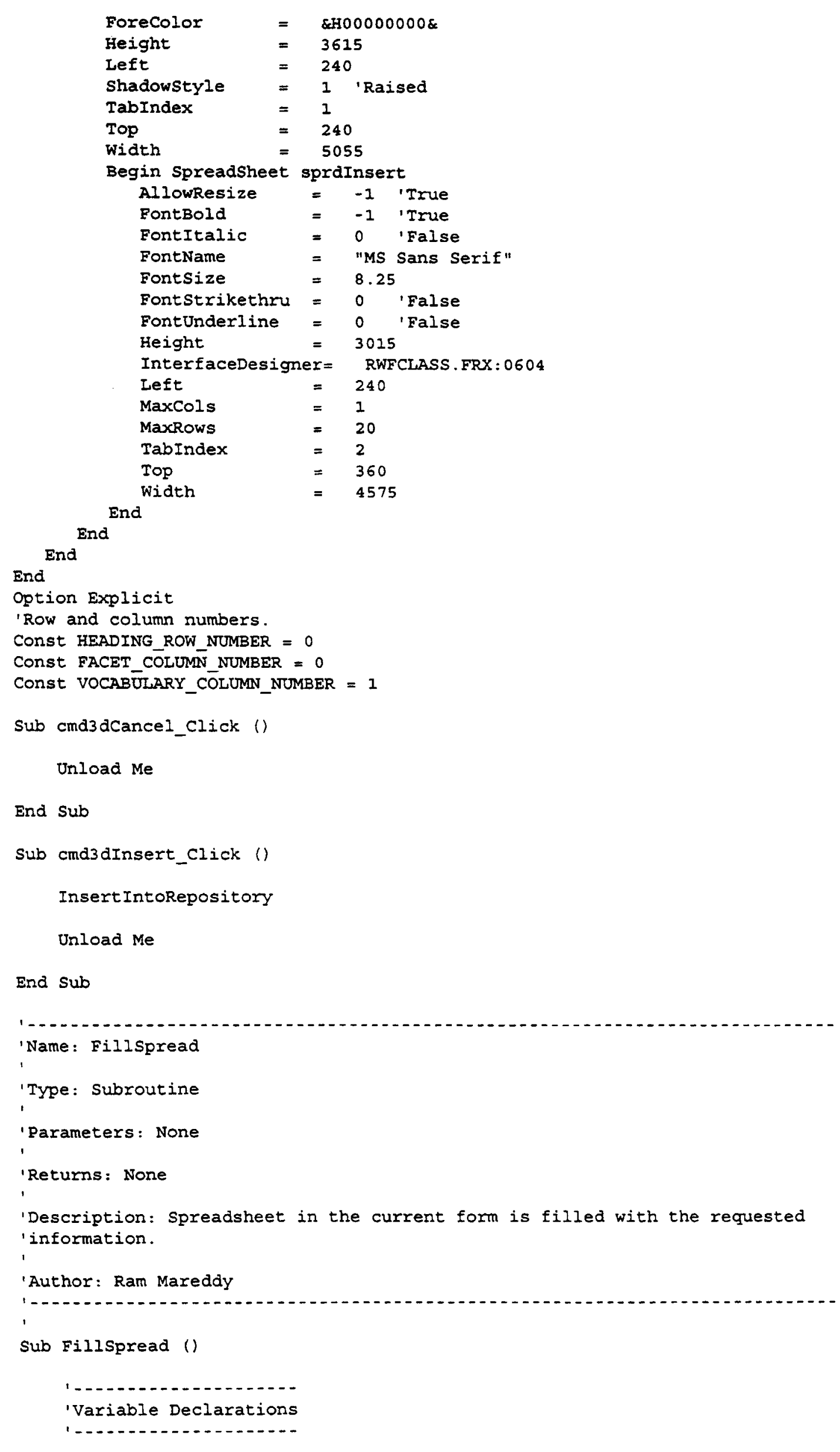


'Number of facets in the system.

Dim nNumpacets As Integer

'Loop counters.

Dim $i$ As Integer

Dim $j$ As Integer

'Number of columns in the spread.

Const NUM_COLUMNS_IN_SPREAD $=2$

'Get the number of facets in the system
nNumfacets = GetNumFacets()

1 -...-.--1-

' Set the maximum number of rows in the spread

'

sprdinsert . MaxRows $=$ nNumFacets

'-

' Clear the Spread

1 .................

FOI $i=0$ TO NUM_COLUMNS_IN_SPREAD

For $j=1$ To nNumFacets

sprdinsext. Row $=j$

sprdinsert. Col $=i$

sprdinsert. Text $=" 1$

Next

Next

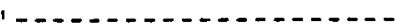

' Fill facets in search

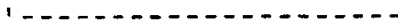

FillFacetsInInsext

'Fill Vocabulary in search

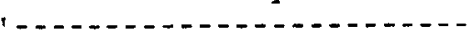

'Initially put ALI in all the vocabulary.

For $j=1$ To nNumFacets

sprdinsert. Row $=j$

sprdInsert.COI = VOCABULARY_COLUMN_NUMBER

sprdInsert. Text $=$ "ALI"

Next $j$

End Sub

Sub Form_Load ()

1..............

' Center the form

1........-......-

Centerform Me

$1-. .-1 .--$

' Set spread

setspread 
' Fill Spread

1............

Fillspread

End $S u b$

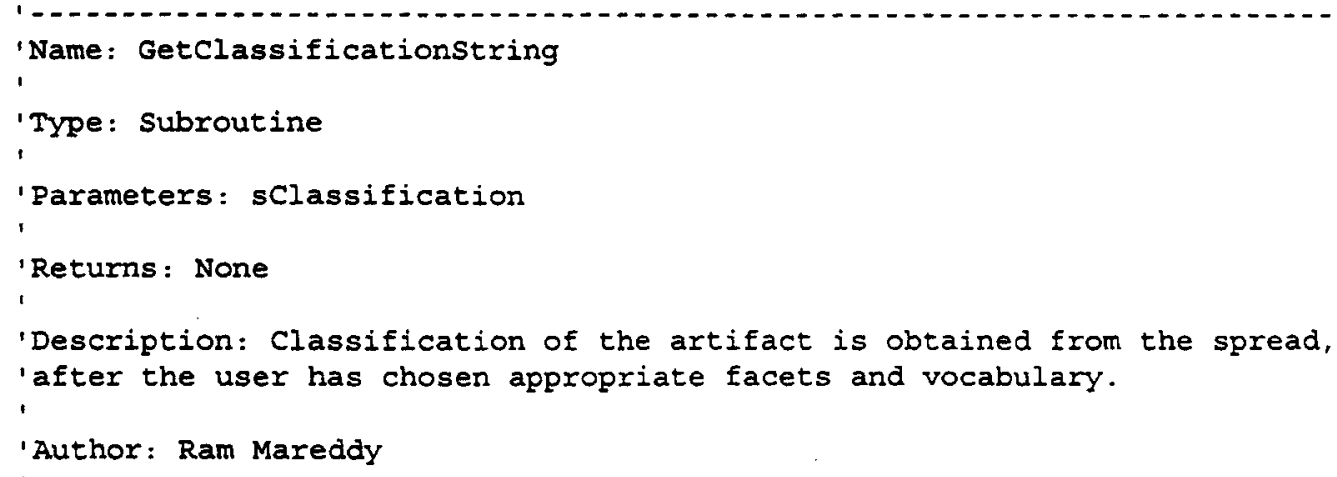

Next $i$

End Sub

'Name: GetFacetString

'Type: Subroutine 


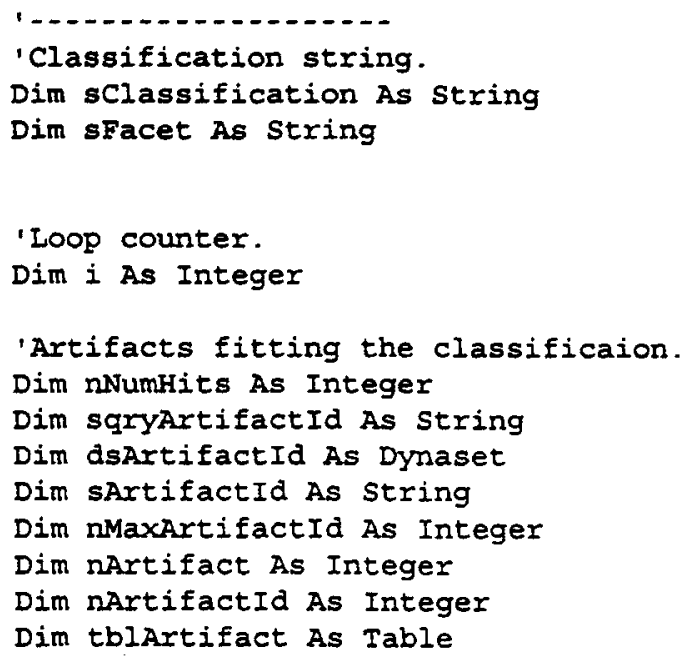




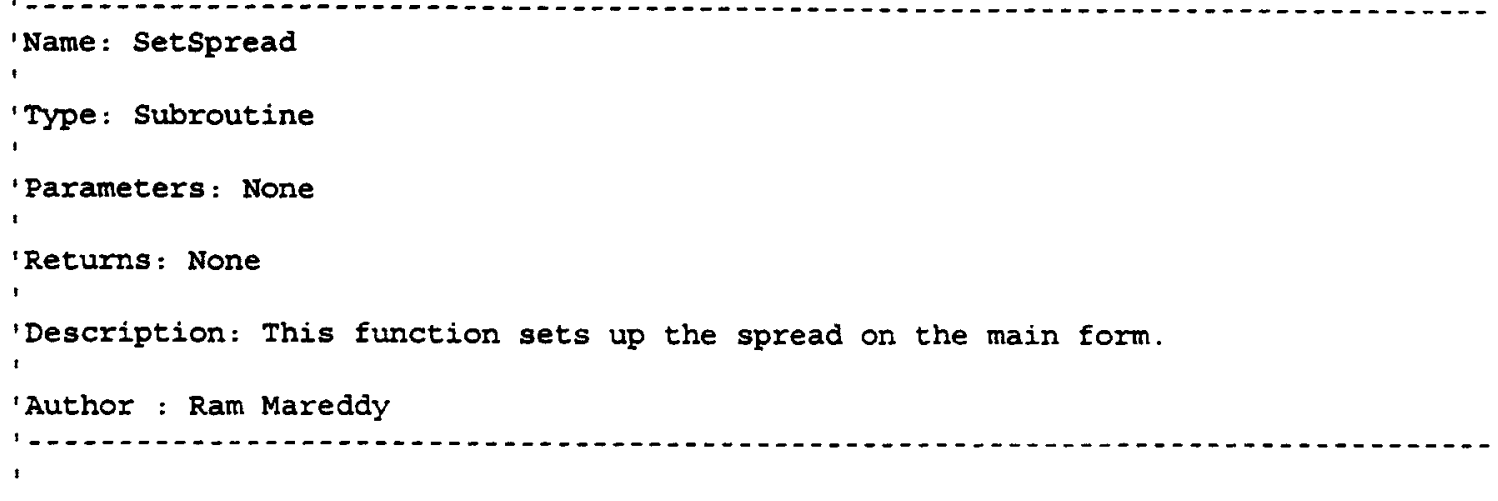

Sub setspread ()

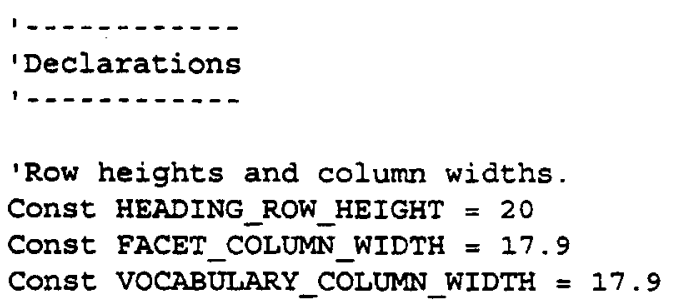




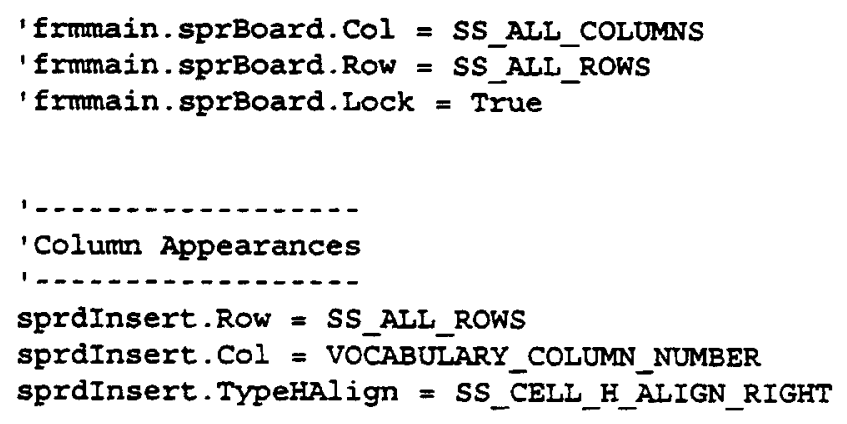

End $\mathrm{sub}$

'Name: sprdSearch_click

Type: subroutine

'Parameters: Col is the column of the selected cell, Row is the row of the 'selected cell.

'Returns: None

'Description: If the cell is in the vocabulary column, then put a combo box 'with the related vocabulary as contents.

End sub

'Name: sprdSearch_LeaveceII

'TYpe: subroutine

'Parameters: Col is the column of the selected cell, Row is the row of the 'selected cell, NewCol is the column of the cell to which the selection is 'moving to and NewRow is row of the same cell.

'Returns: None

'Description: If the cell is in the vocabulary column, then take out the 'combo box that is presently in that cell.

'Author: Ram Mareddy

'A

Sub spraInsert_LeaveCell (Col As Long, Row As Long, NewCol As Long, NewRow As Long, Cancel As Integer)

- Variable Declarations

'. -....................

' Column which contains the vocabulary terms.

Const VOCABULARY_COLUMN_NUMBER = 1 
COnSt SYNONYM_COLUMN_NOMBER $=2$

Make the cell type text

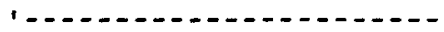

If $(\mathrm{CO})=$ VOCABULARY_COLUMN_NUMBER) Or (COI $=$ SYNONYM_COLUMN_NUMBER) Then sprdInsert. CellTYpe = SS CELI TYPE STATIC TEXT End If sprdInsert. TYpeHAlign $=\bar{S} S_{-} C E \bar{L} L_{-} H_{-} \overline{A L I G N}_{\text {R }} \bar{I} \mathrm{IGHT}$

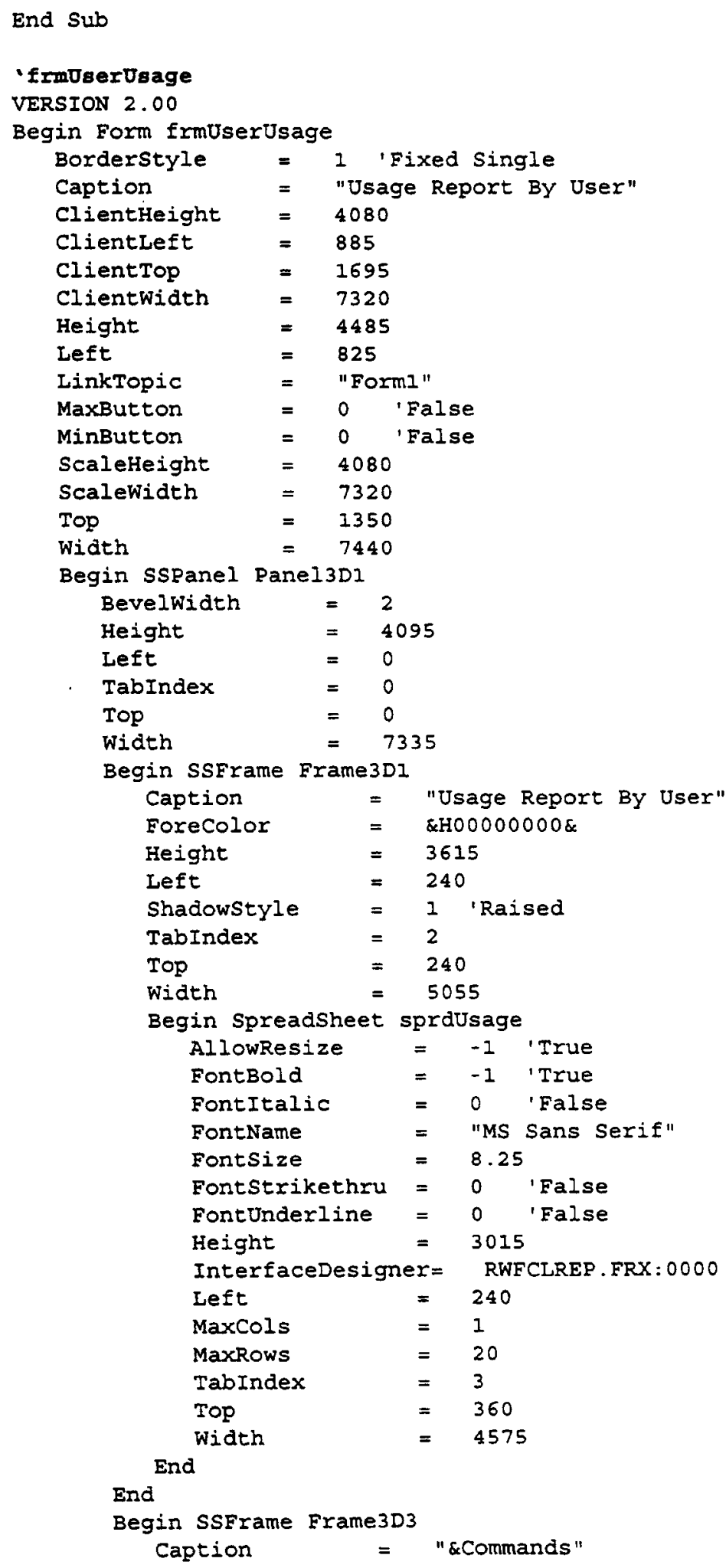




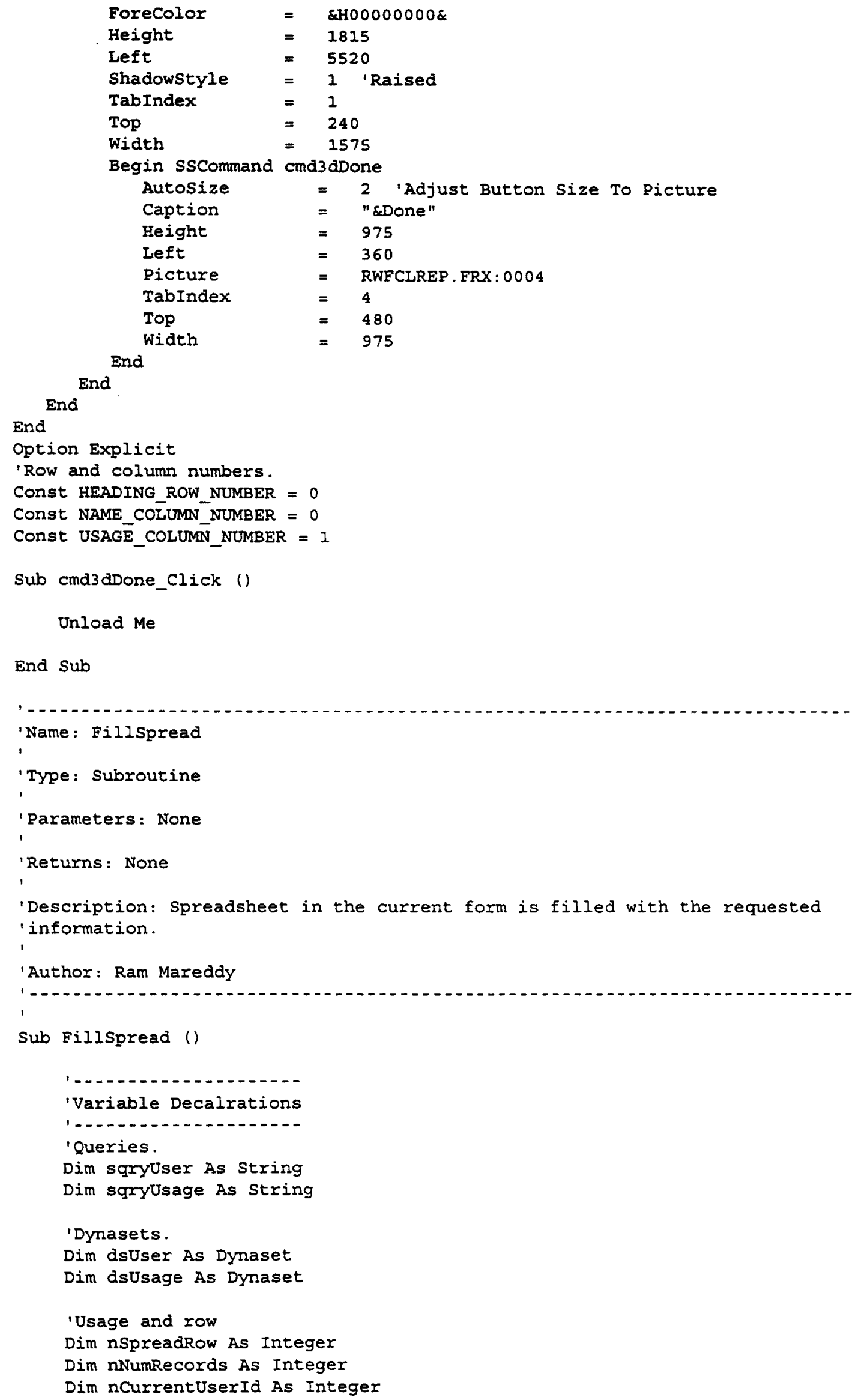




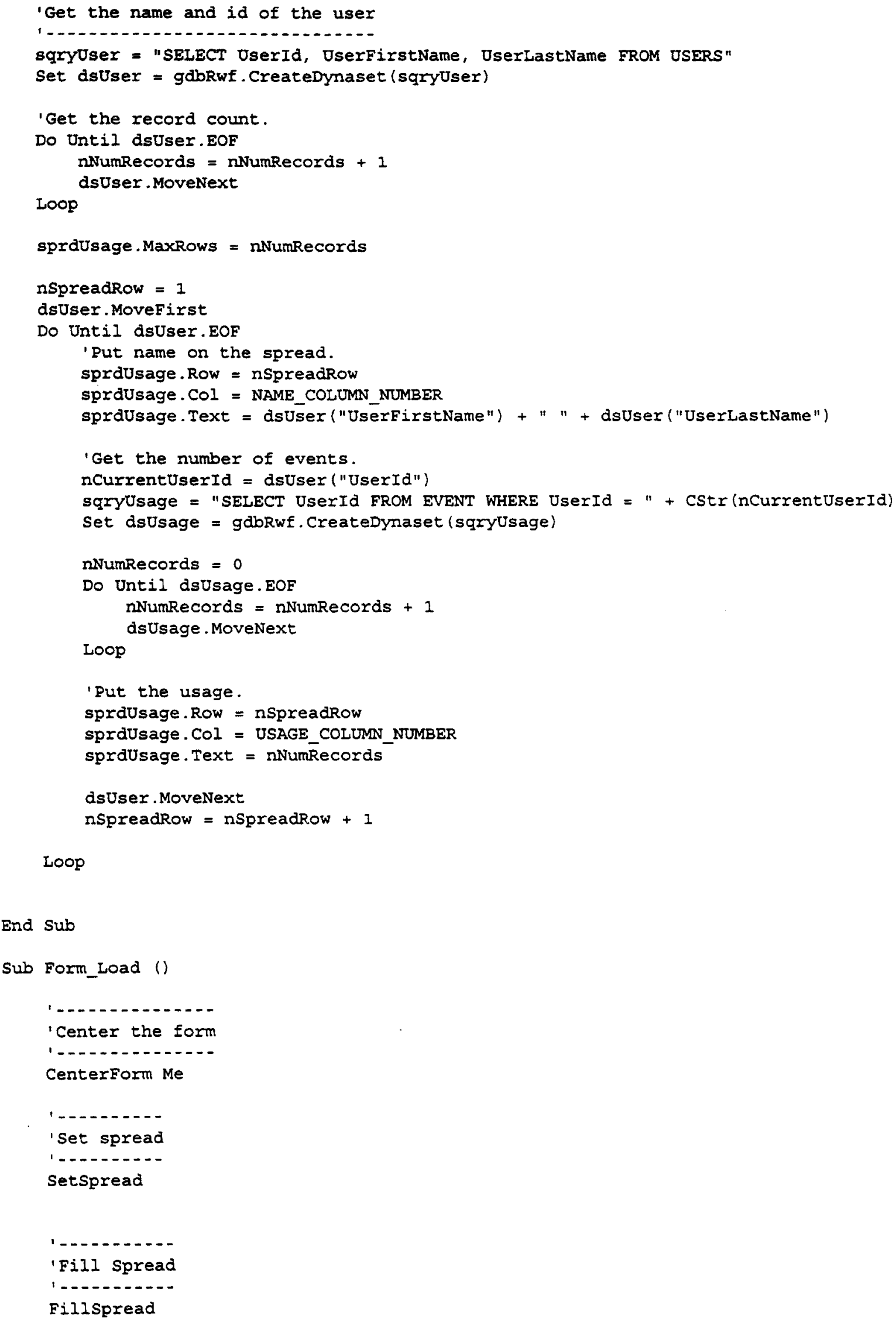




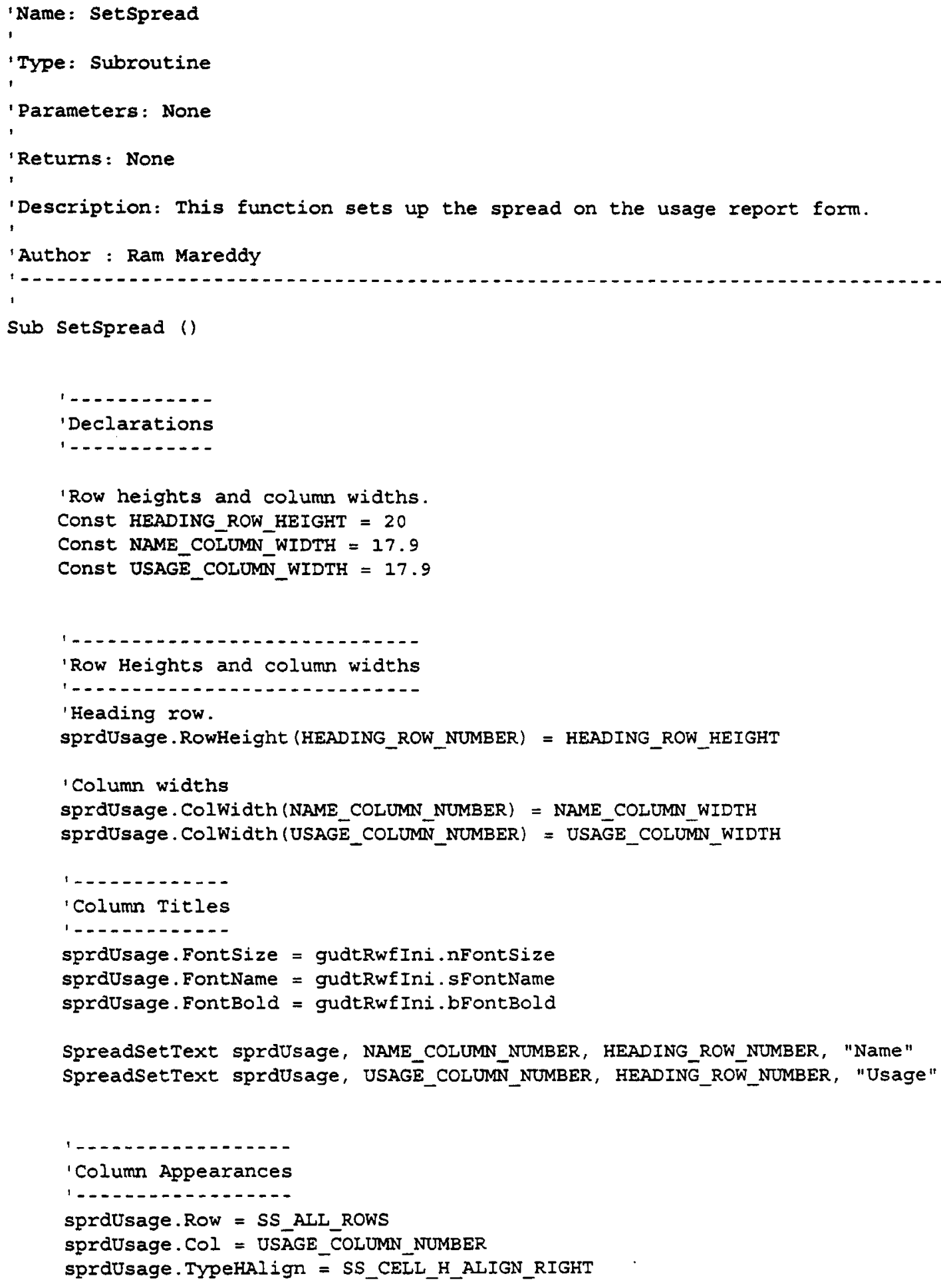

End sub

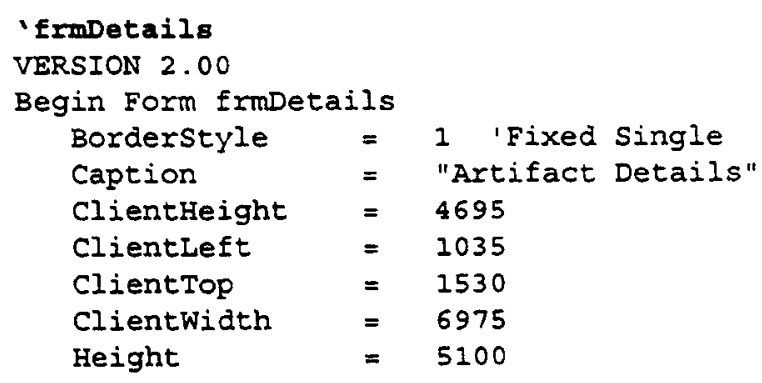




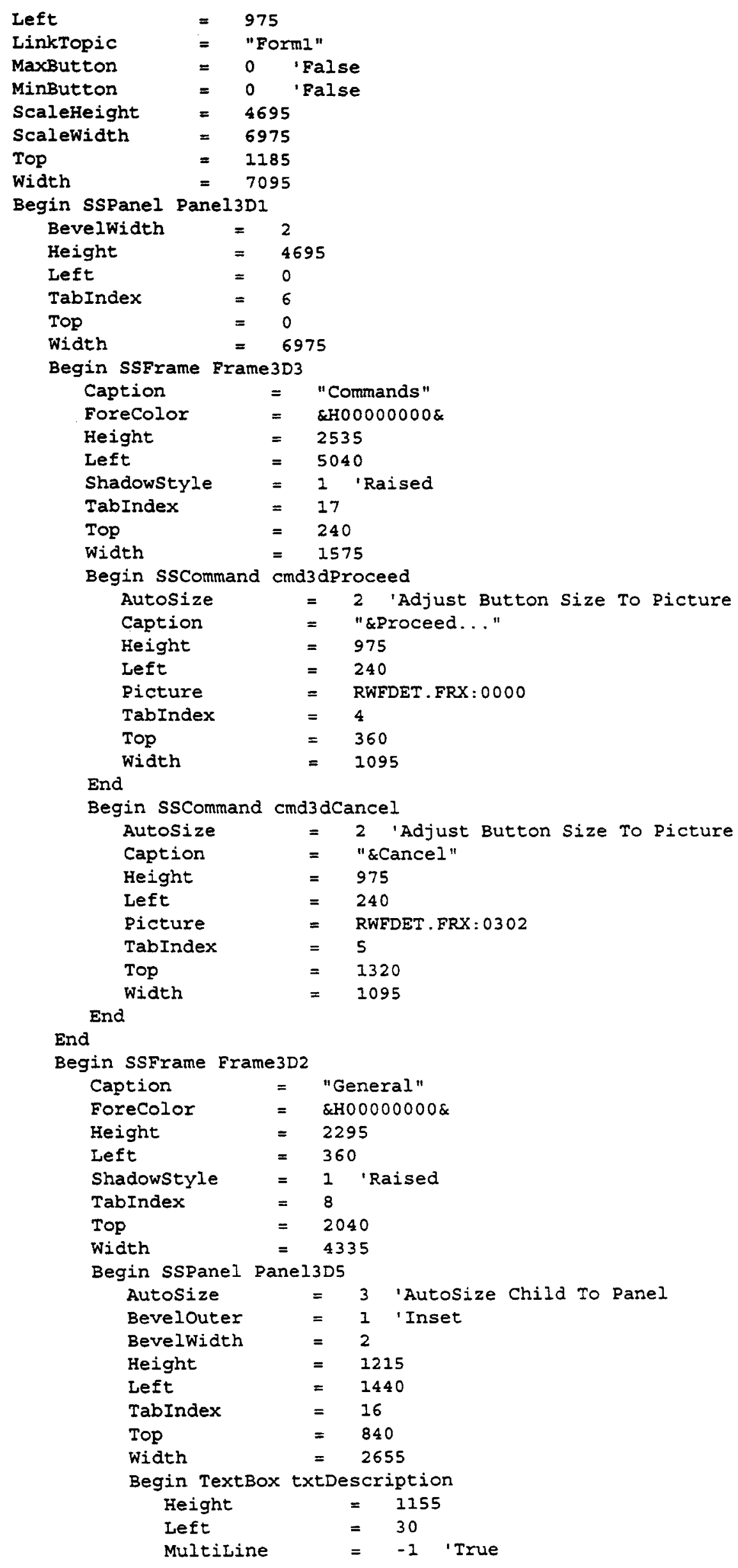




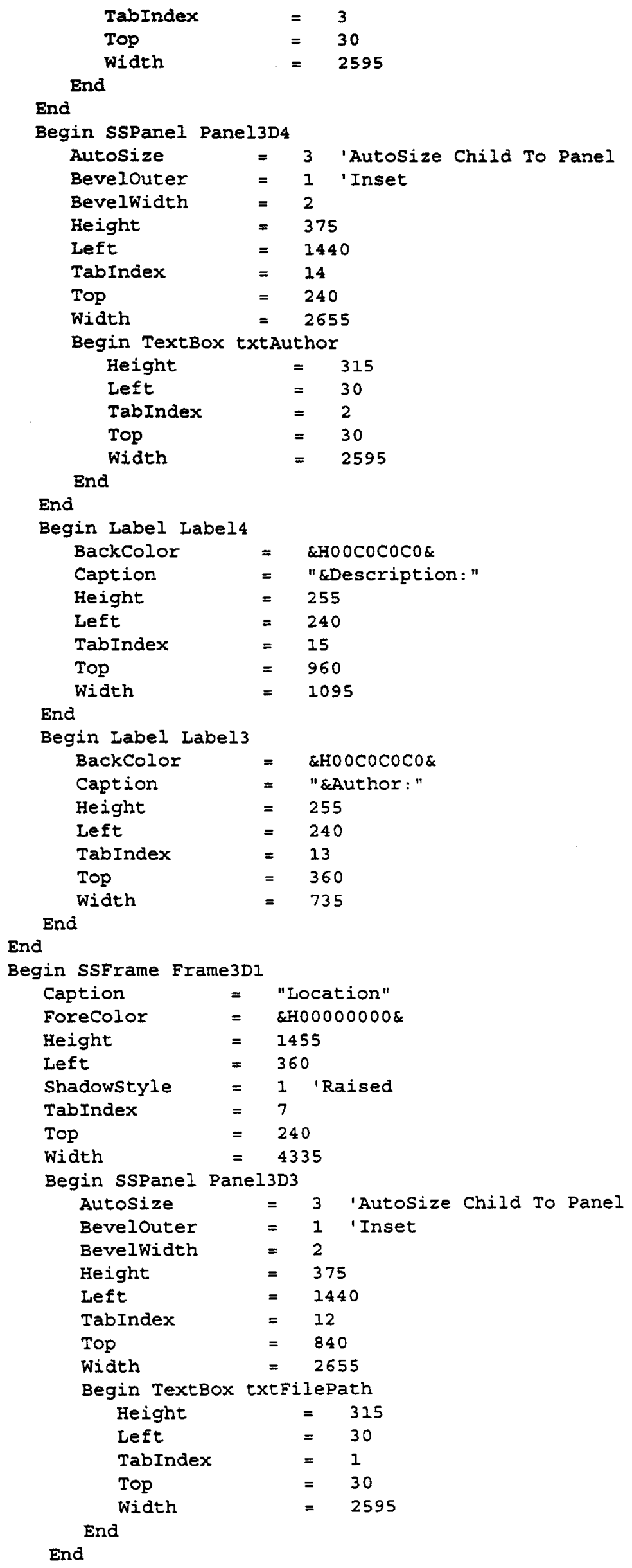




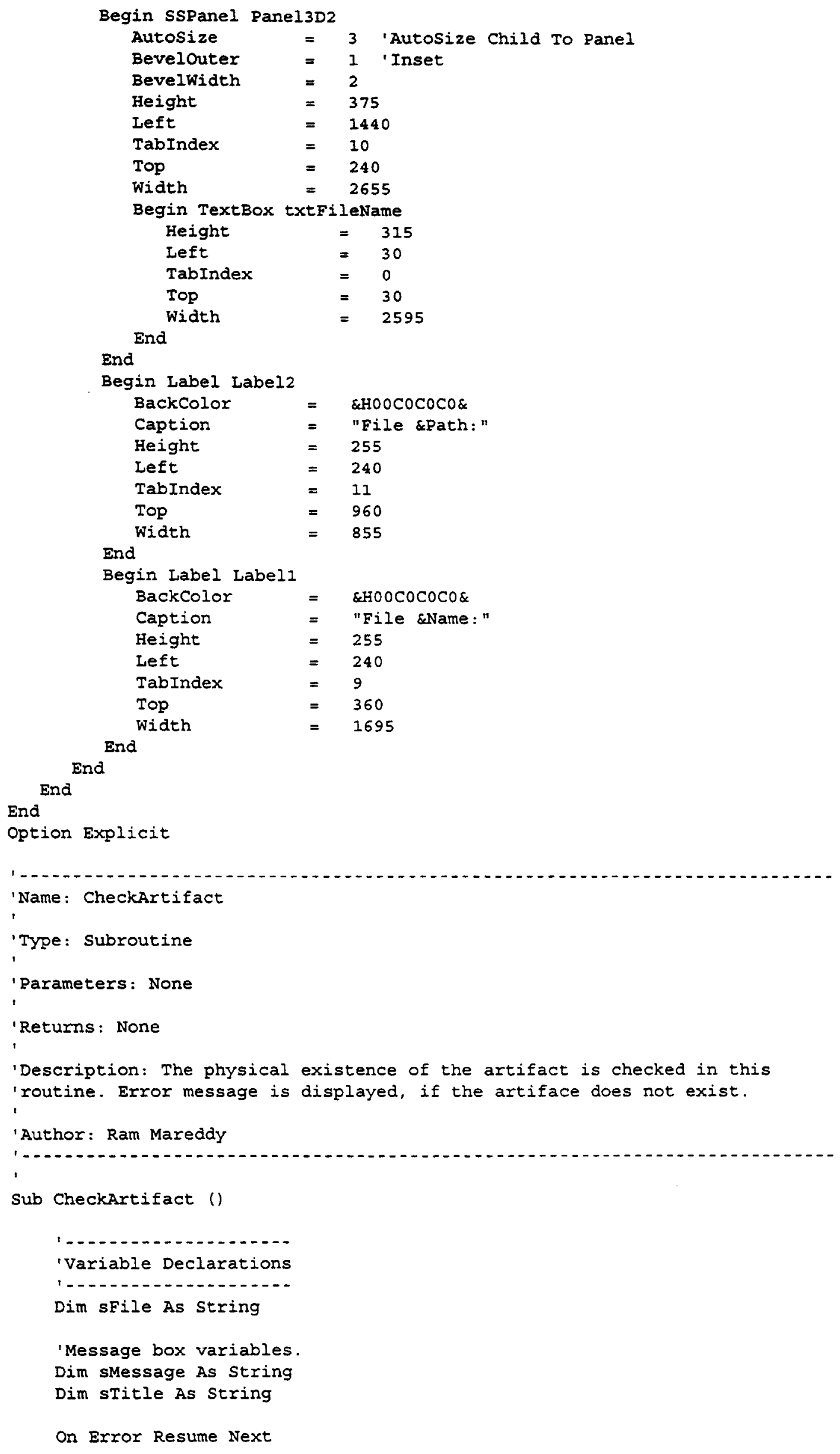




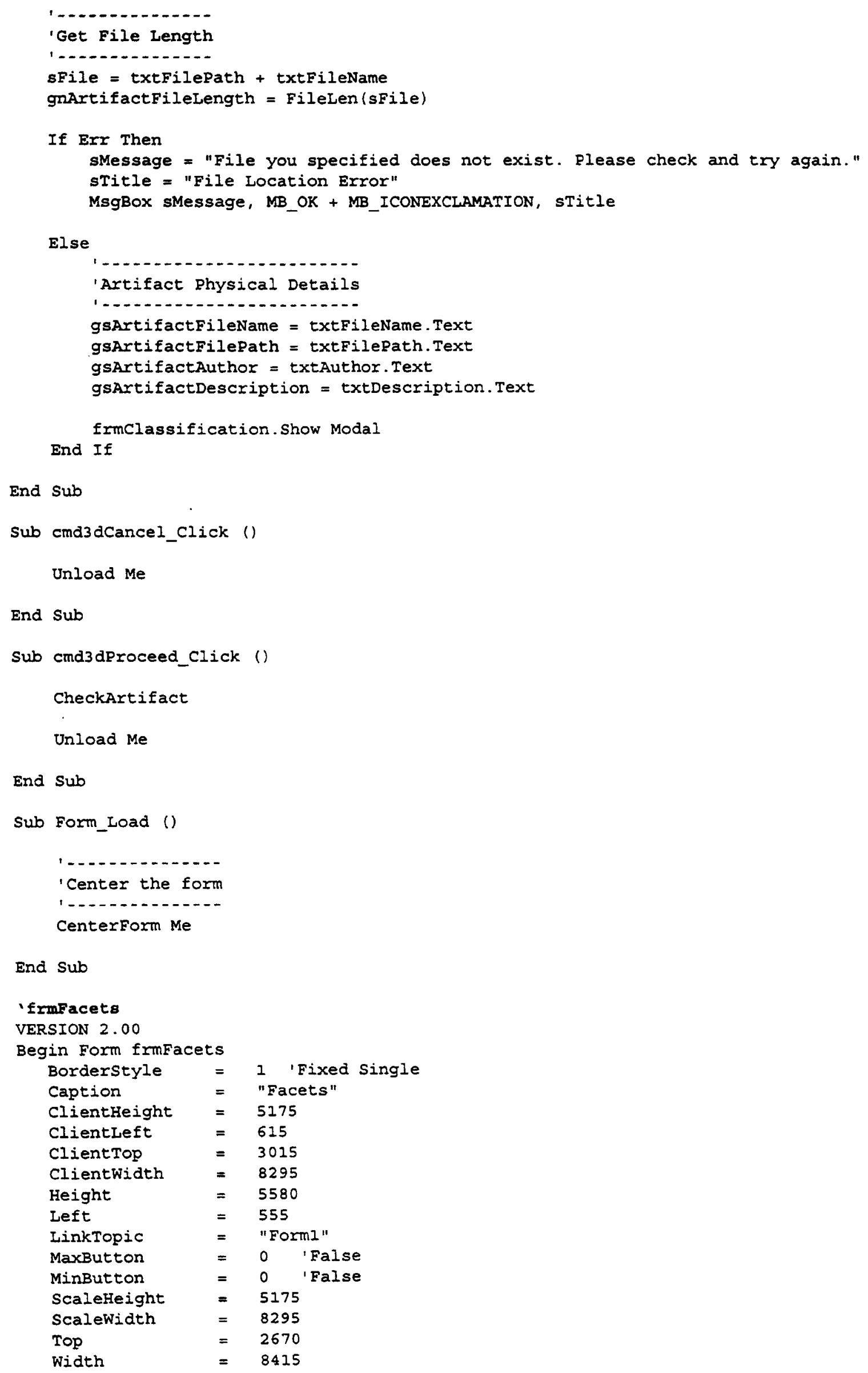




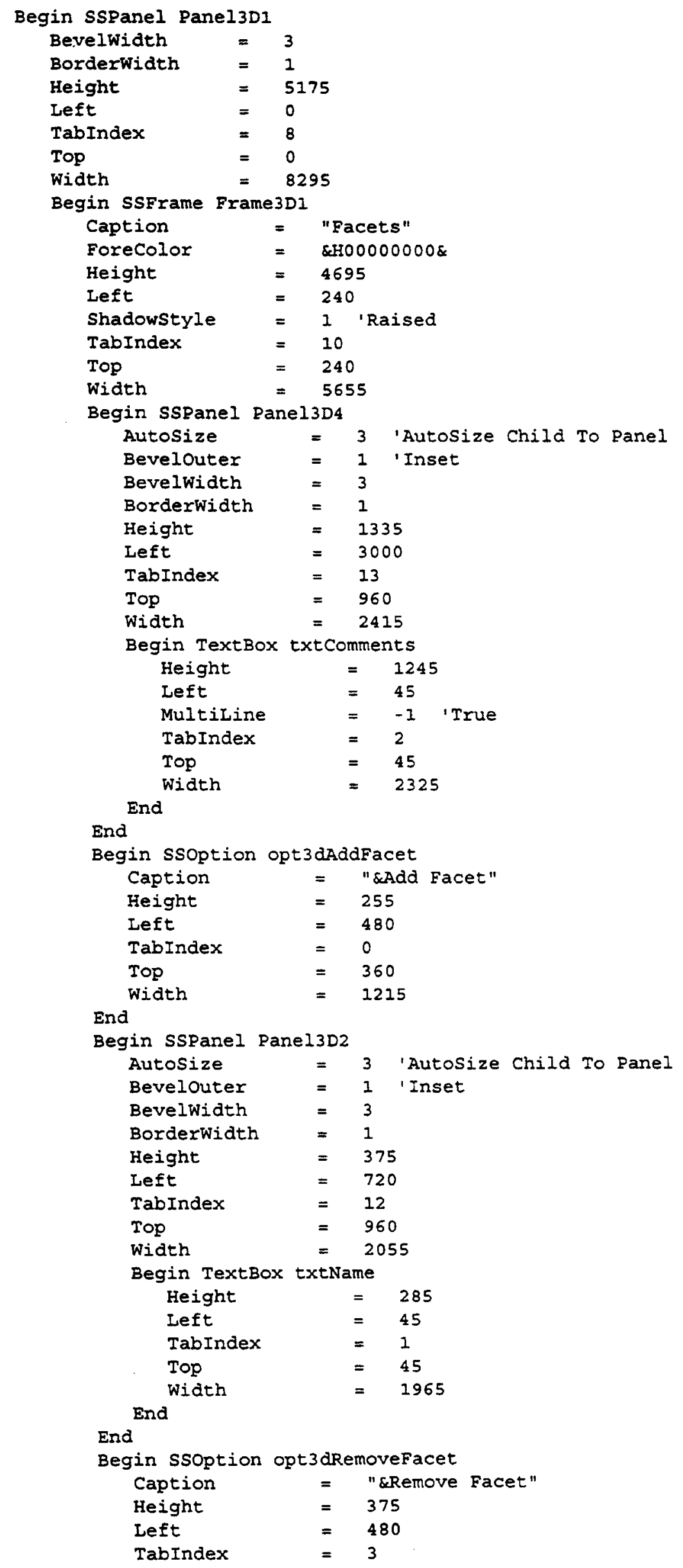




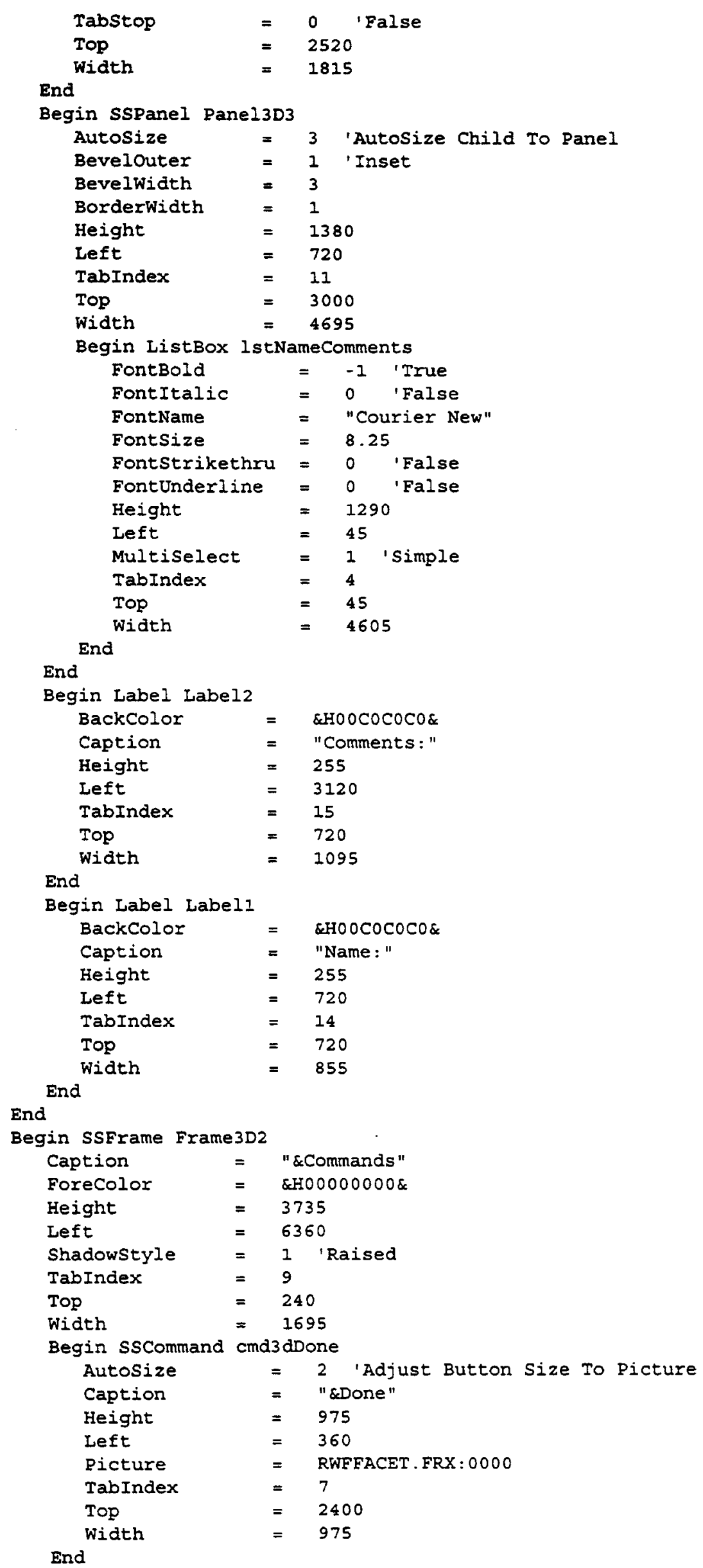




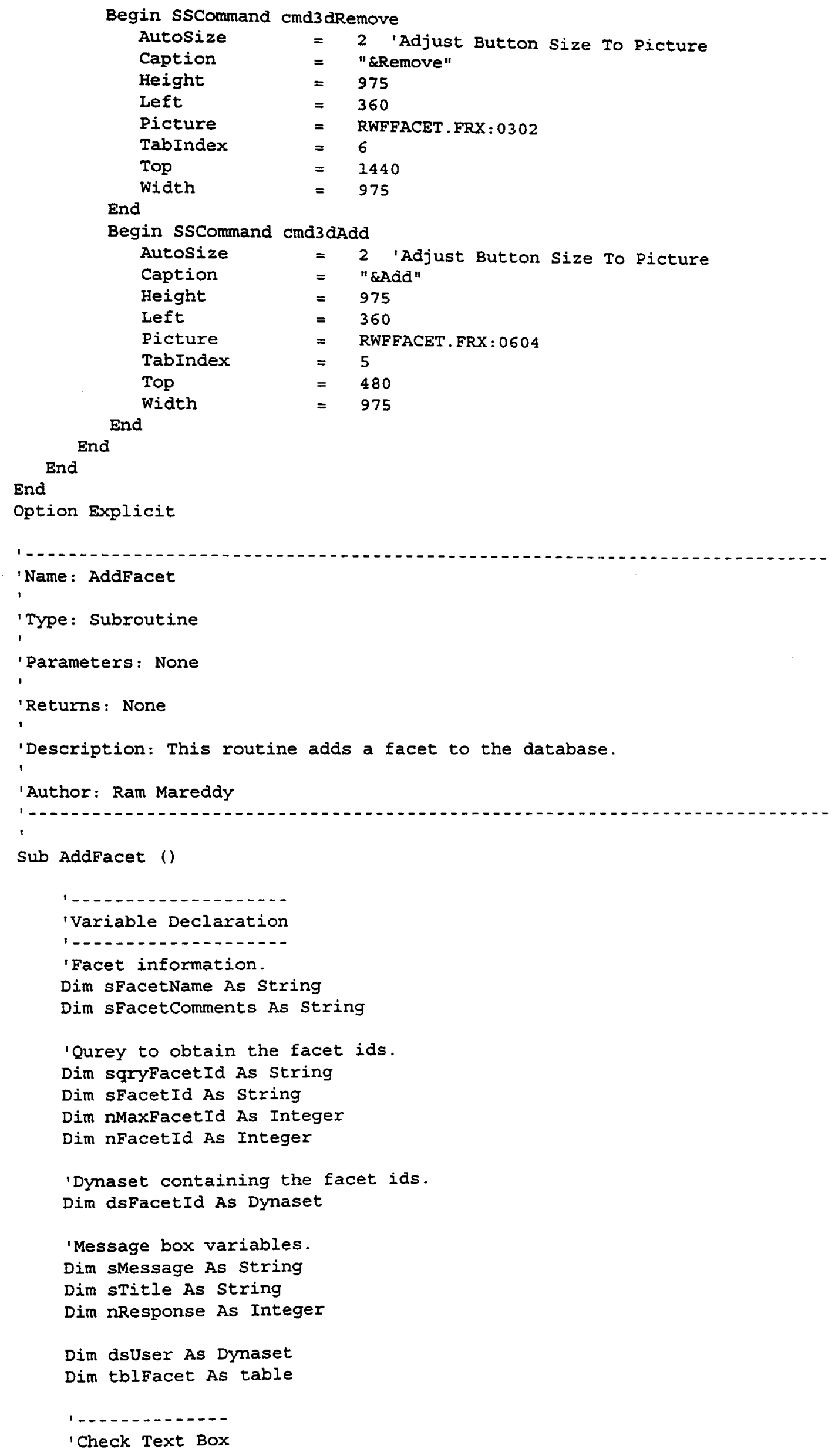




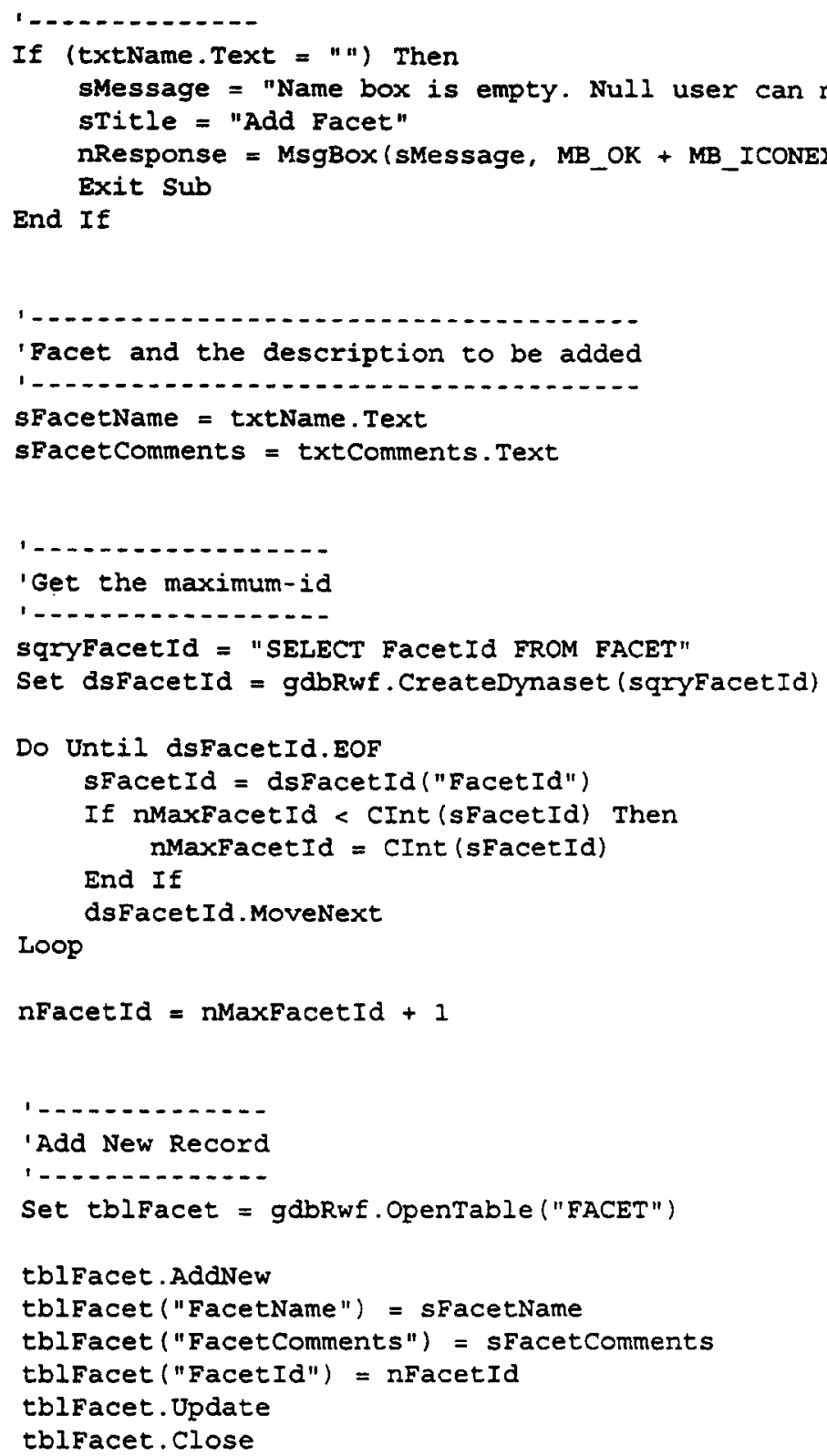


txtName.SetFocus

End sub

Sub and3dDone_click ()

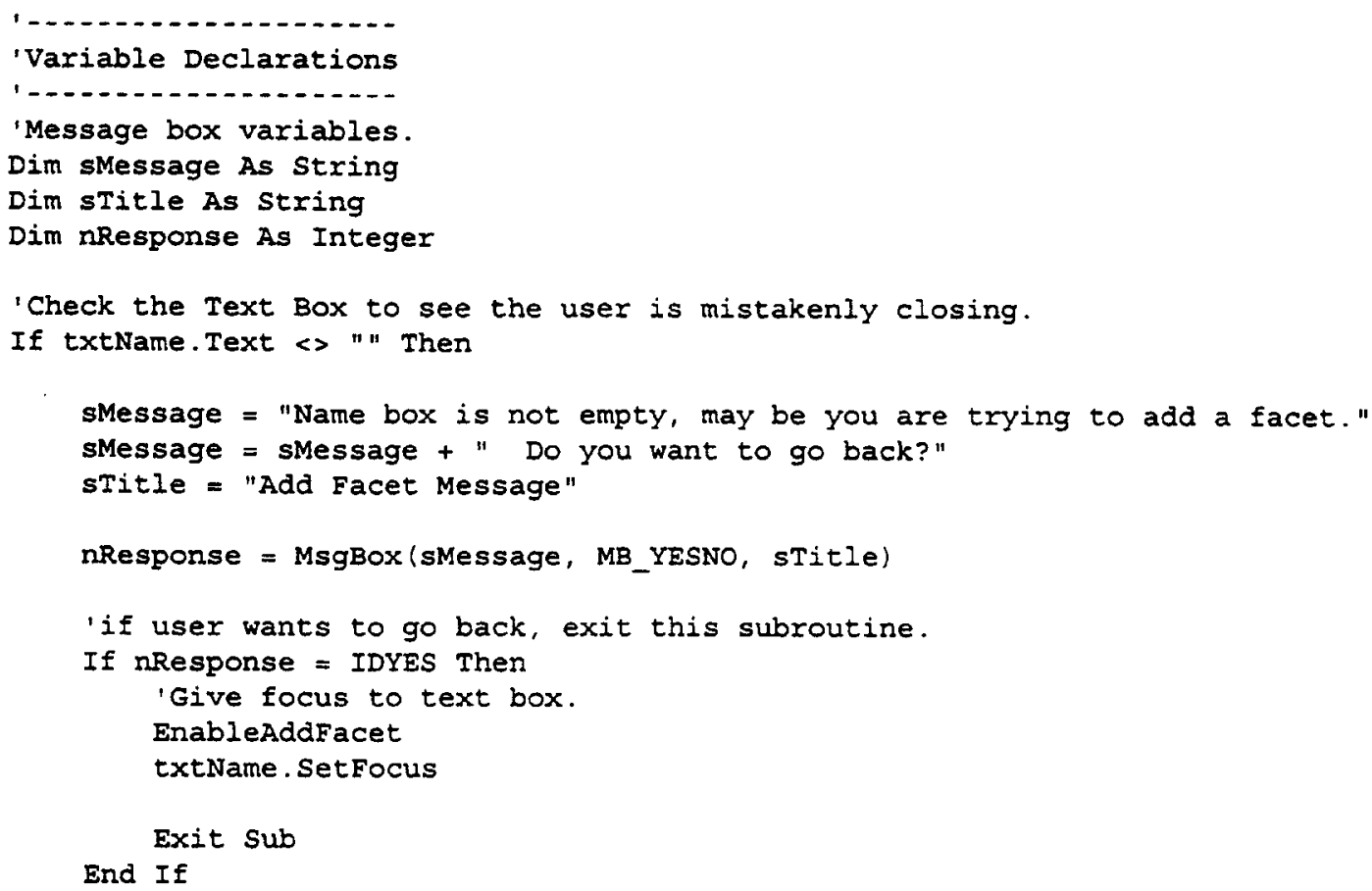

End sub

'Name: EnableAddFacet

Type: Subroutine

Parameters: None

Returns : None

'Description: This function enables all the add facet boxes and buttons, where as the EnableRemoveFacet does the vice versa. 


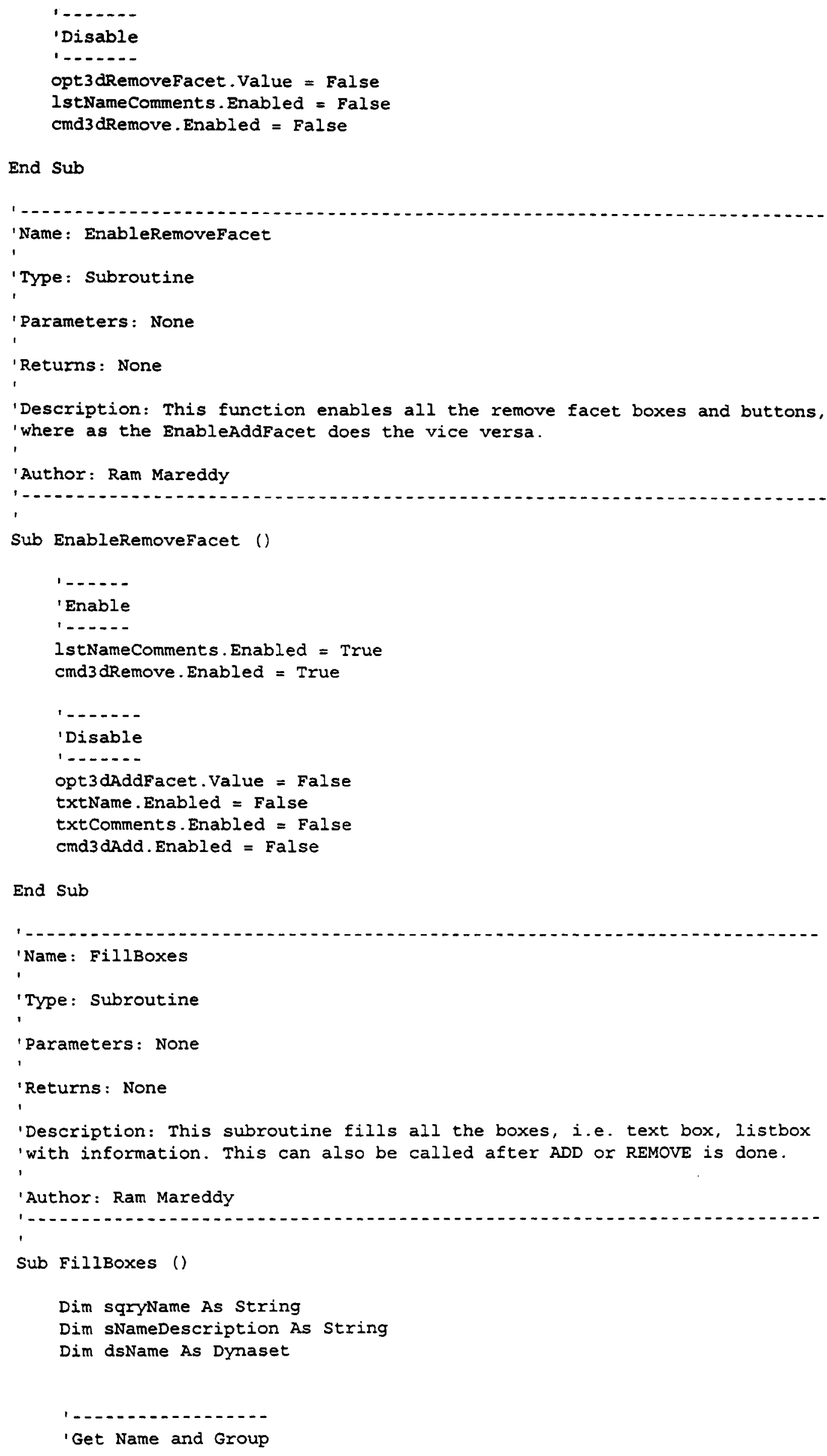

End Sub

Name: FillBoxes

'Type: Subroutine

'Parameters: None

'Returns : None

Description: This subroutine fills all the boxes, i.e. text box, listbox 'with information. This can also be called after ADD or REMOVE is done. 


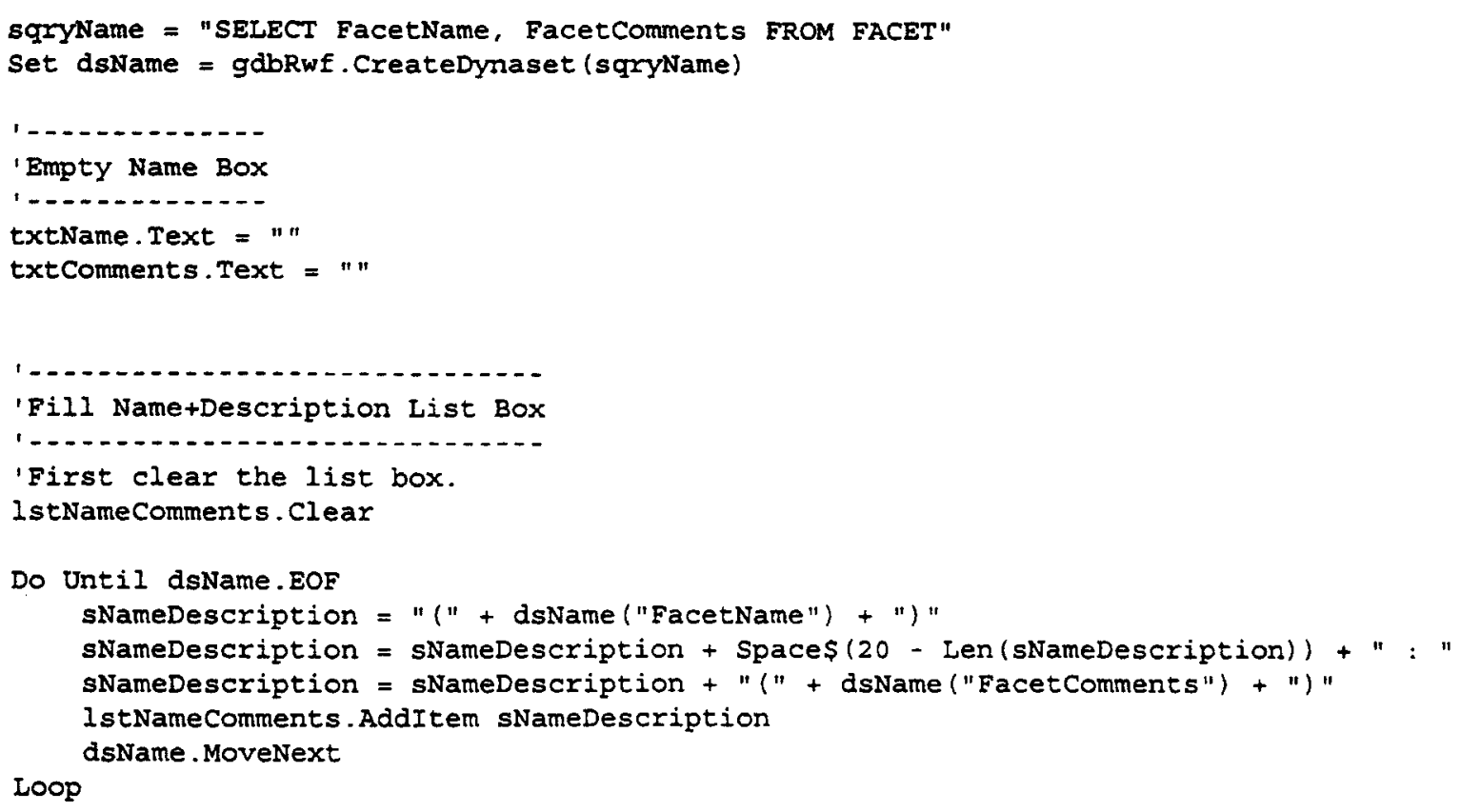

End sub

Sub Form_Load ()

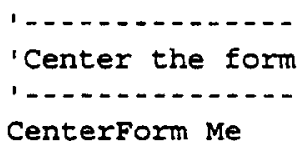

End sub

Sub opt3dAddFacet_Click (Value As Integer)

EnableAddFacet 
'Description: This function removes one or more users selected in the 'remove list box.

'Author: Ram Mareddy

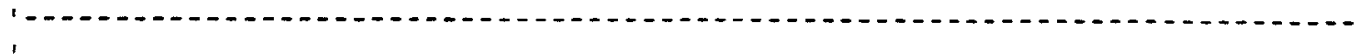

Sub RemoveFacet ()

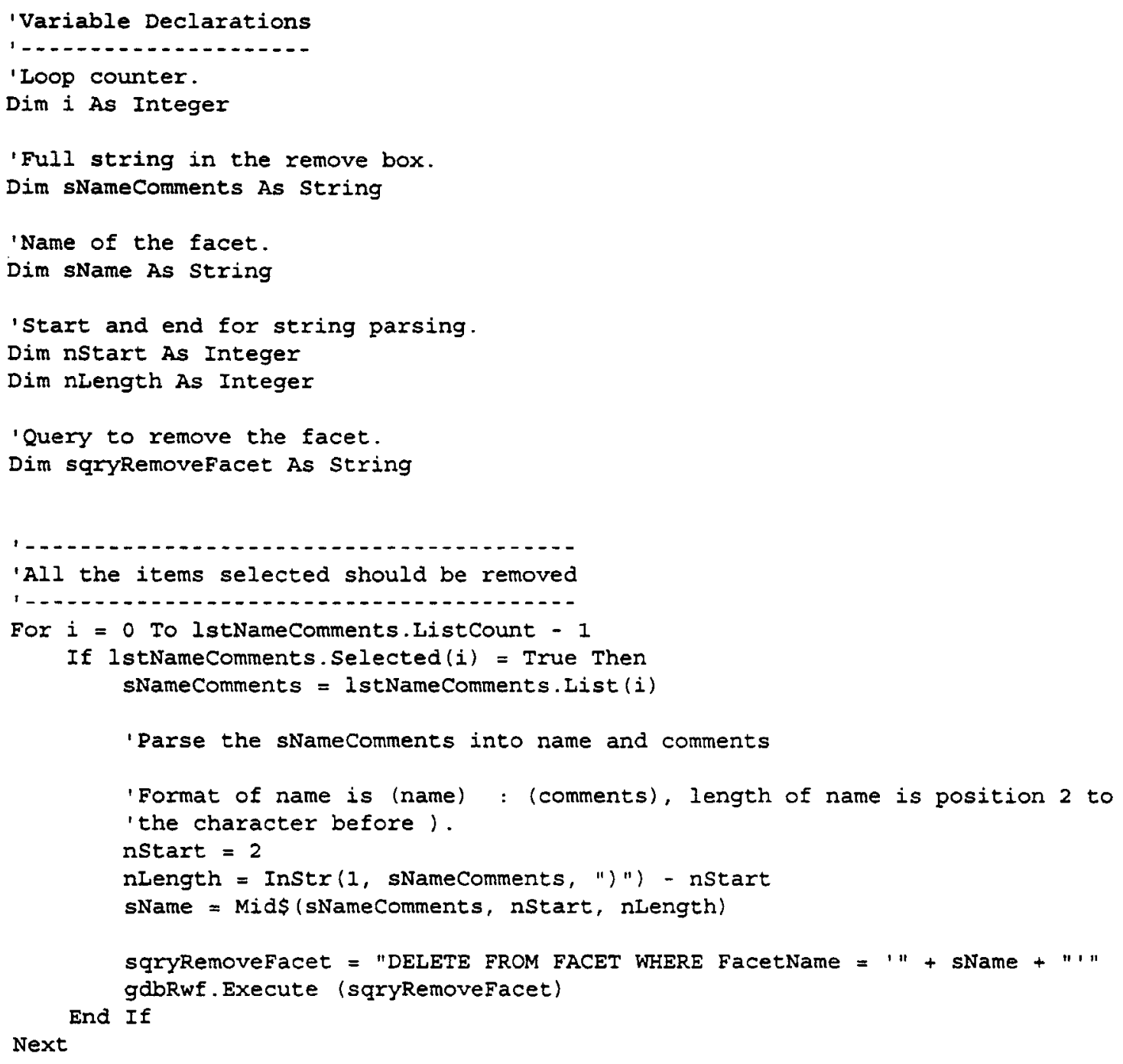

End Sub

ermugin

VERSION 2.00

Begin Form frmLogin

Borderstyle $=1$ 'Fixed single

Caption = "Login" 


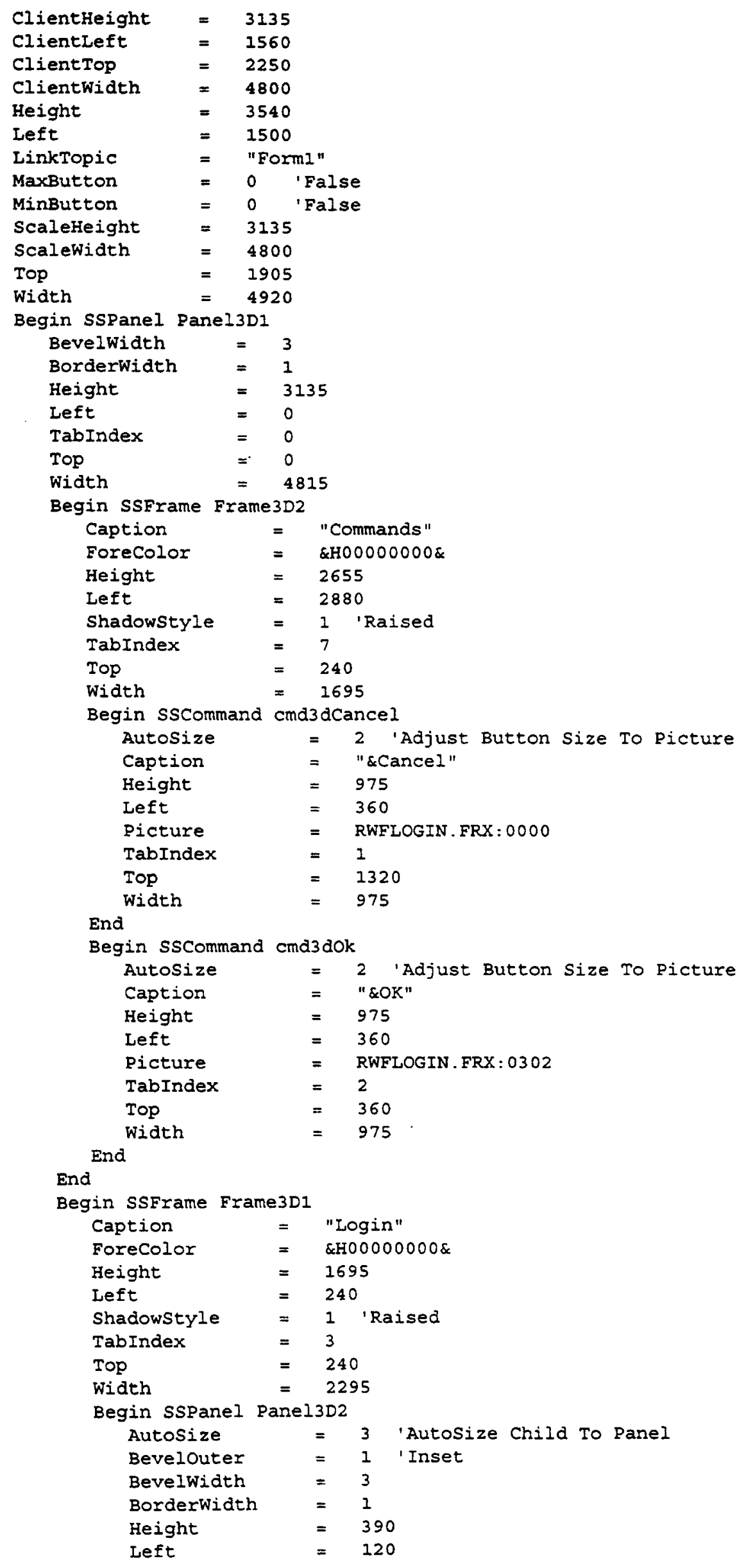




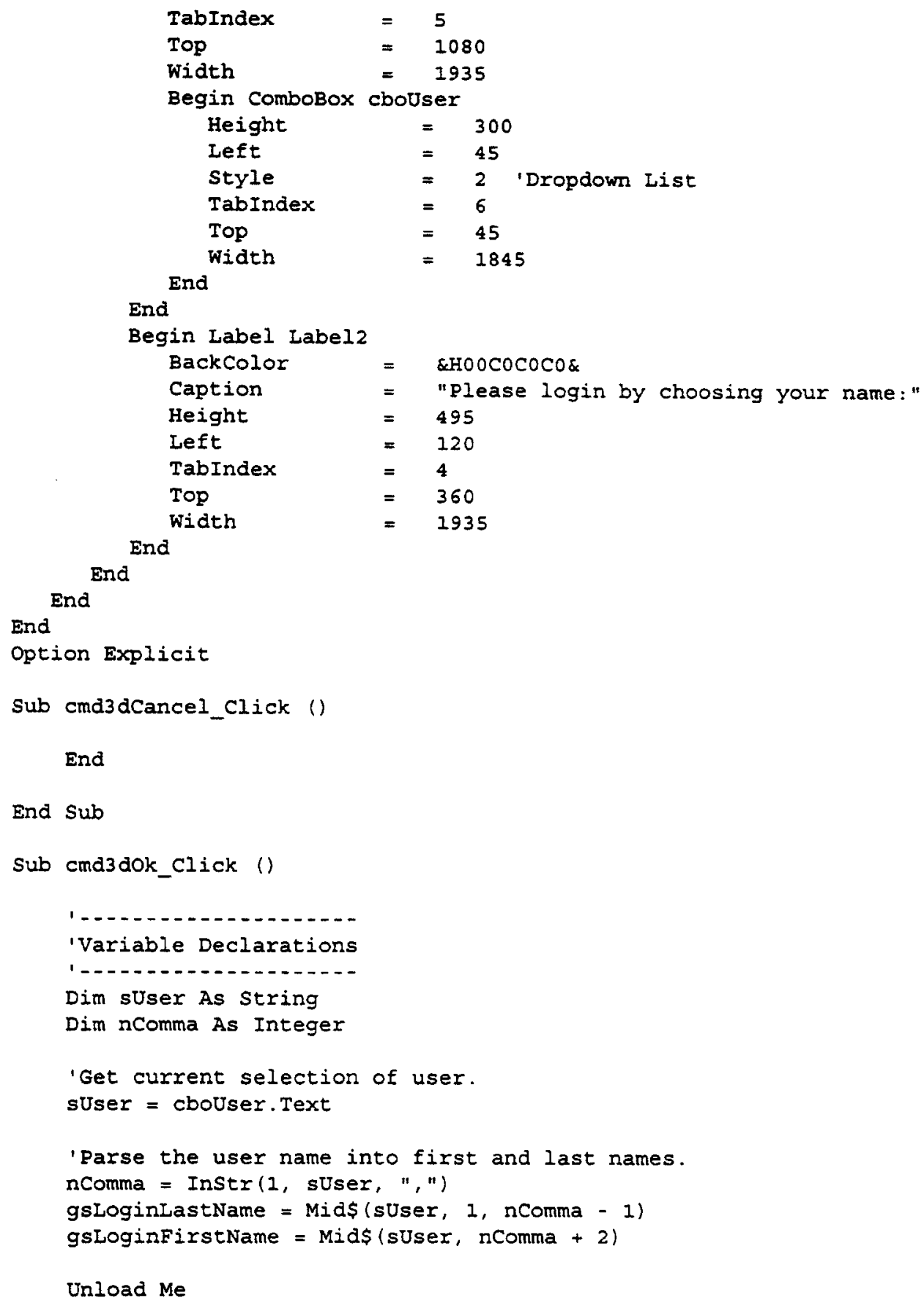




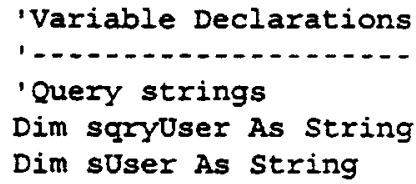

End sub

Sub Form_Load ()

'Put the user names in the combo. FillCombo

End Sub

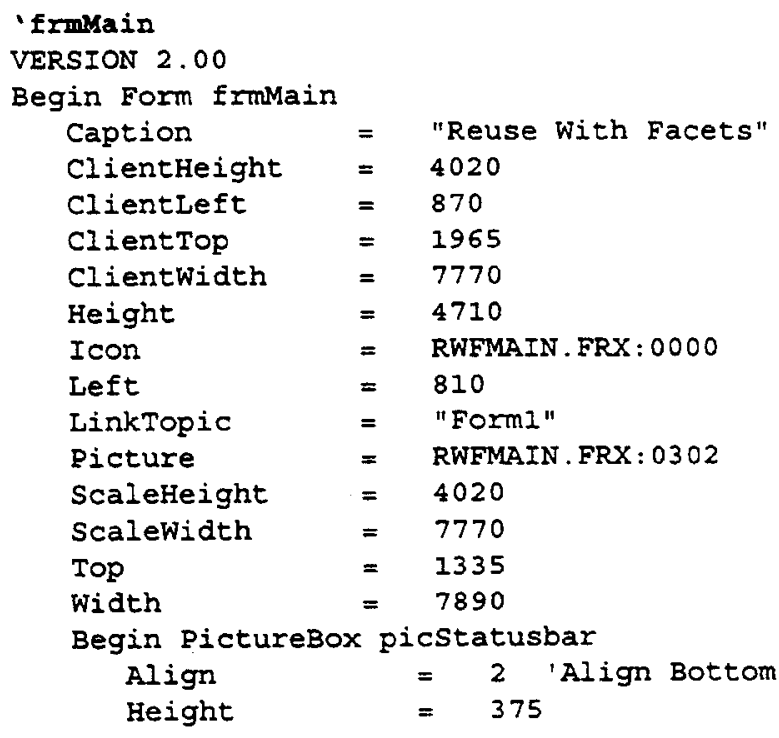




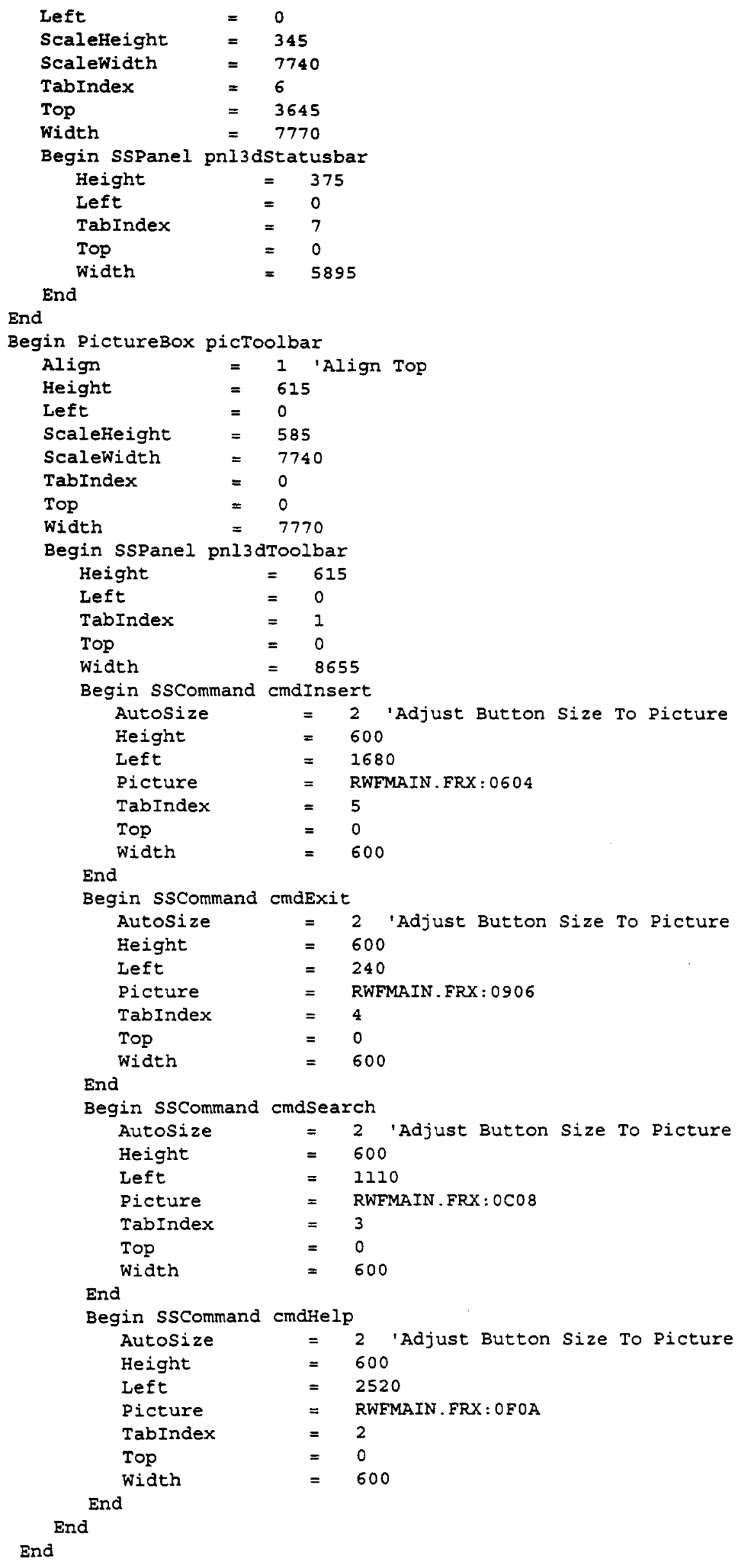




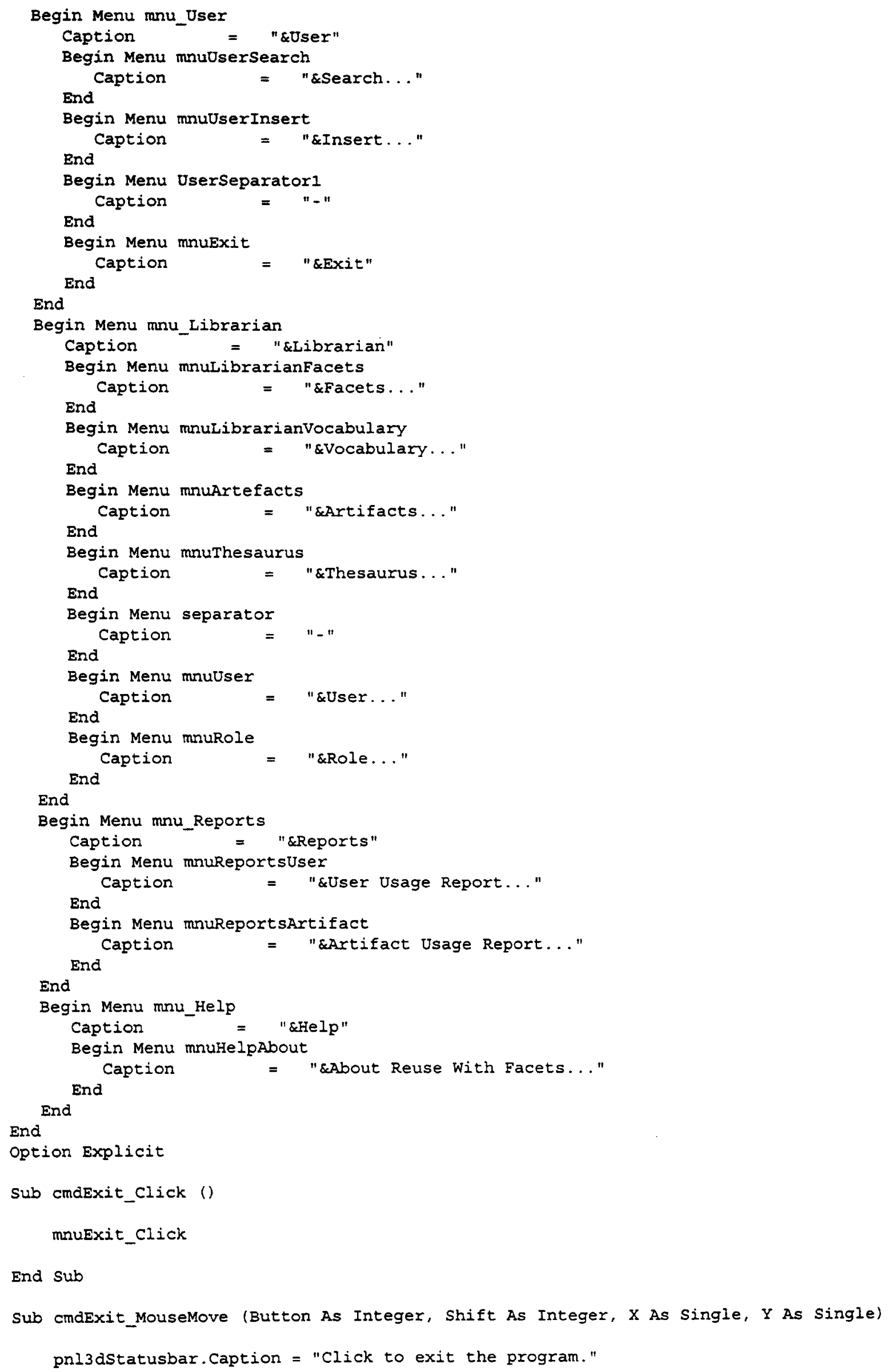




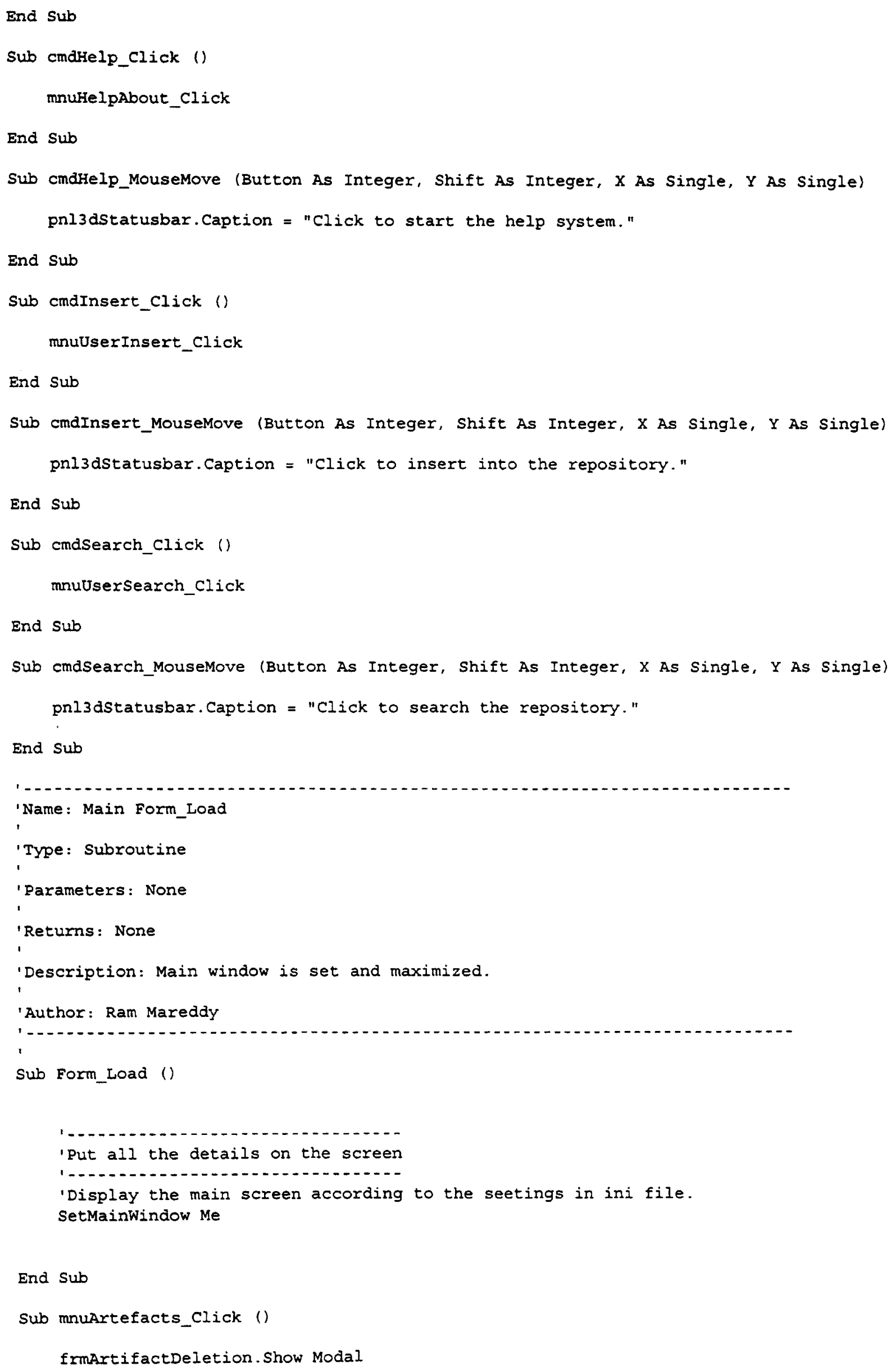


End Sub

Sub mnuExit_Click ()

End

End Sub

Sub mruHelpAbout_click ()

frmabout. Show Modal

End sub

Sub mnulibrarianFacets_click ()

ErmFacets. Show Modal

End Sub

Sub mnulibrarianvocabulary_click ()

frmvocabulary. Show Modal

End Sub

Sub mnuReportsArtifact_Click ()

frmartifactusage. Show Modal

End Sub

Sub mnuReportsuser_click ()

frmUserUsage. Show Modal

End sub

Sub mnuRole_click ()

frmiole. Show Modal

End sub

Sub mnuThesaurus_click ()

frmThesaurus. Show Modal

End sub

Sub mnuUser_Click ()

frmuser. Show Modal

End sub

Sub mnuUserInsert_Click ()

frmDetails. Show Modal

End Sub

'Name: mnuUsersearch_click

Type: subroutine 
'Parameters: None

'Returns: None

'Description: Search command selected.

'Author: Ram Mareddy

' -

sub mnuUsersearch_click ()

Ermsearch. Show Modal

End sub

Sub picstatusbar_Resize ()

pnl3astatusbar. Left $=0$

prl3dStatusbar. Top $=0$

pnl3astatusbar. Height = picstatusbar. Height

pnl3dstatusbar.Width = picstatusbar.Width

End Sub

Sub picToolbar_Resize ()

'Whenever the picture box is resized, resize the panel also.

pnl3dToolbar. Left $=0$

pnl3dToolbar. Top $=0$

pnl3 dToolbar.Height = pictoolbar.Height

pnl3dToolbar. Width = picToolbar. Width

End Sub

Sub pnl3dToolbar_MouseMove (Button As Integer, Shift As Integer, X As Single, Y As Single)

pnl3dStatusbar. Caption $="$ "

End sub

- ETmRole

VERSION 2.00

Begin Form frmRole

Borderstyle $=1$ 'Fixed single

Caption

$=$ "Role"

ClientHeight $\quad=5175$

ClientLeft $=240$

Clientrop $=1590$

Clientwidth $\quad=8295$

Height $=5580$

Left $=180$

LinkTopic = "Forml"

MaxButton $\quad=0$ ifalse

MinButton $=0$ 'False

ScaleHeight $=5175$

Scalewidth $=8295$

Top $=1245$

Width $=8415$

Begin SSPanel Panel3D1

Bevelwidth = 3

Borderwidth $=1$

Height $=5175$

Left $=0$

TabIndex $=8$

Top $=0$

Width $\quad \mathbf{8 2 9 5}$ 


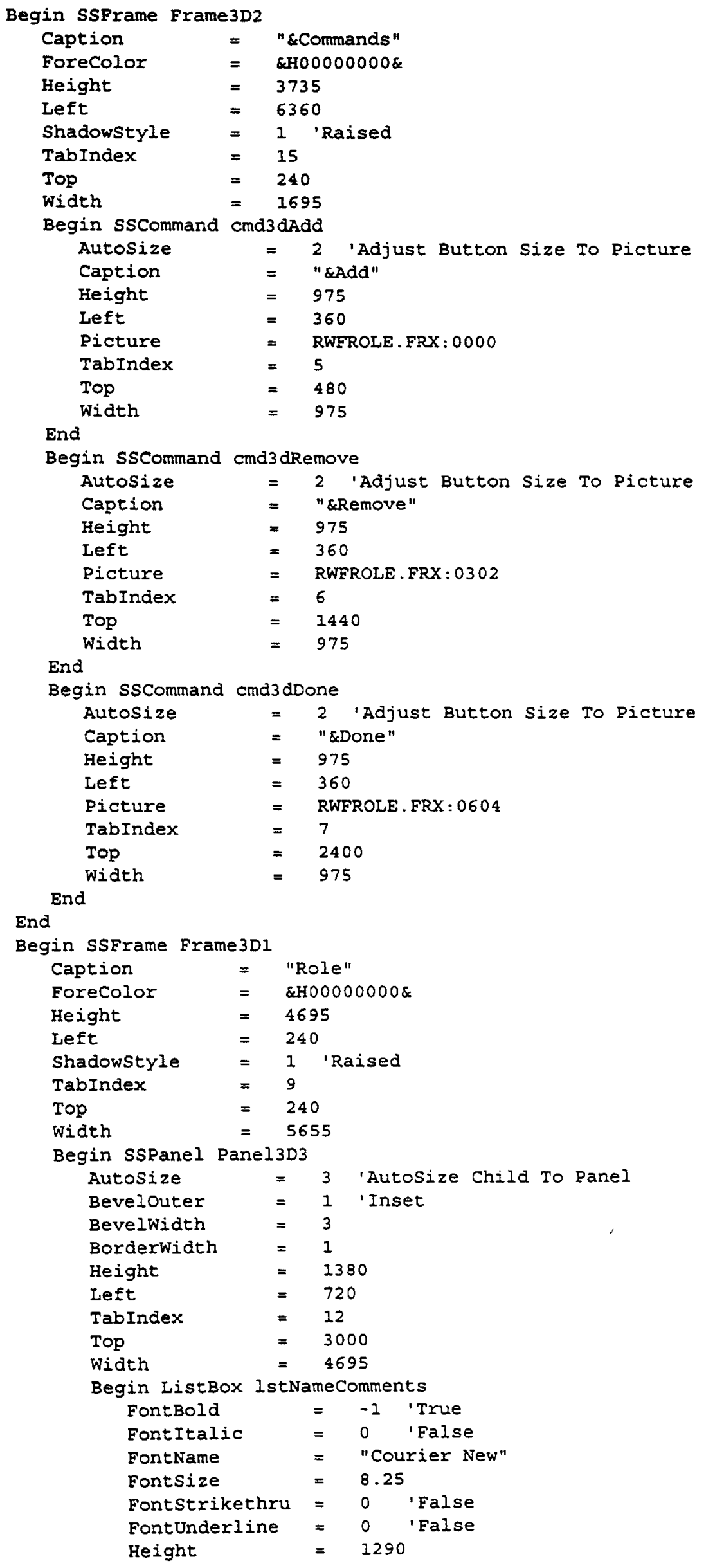




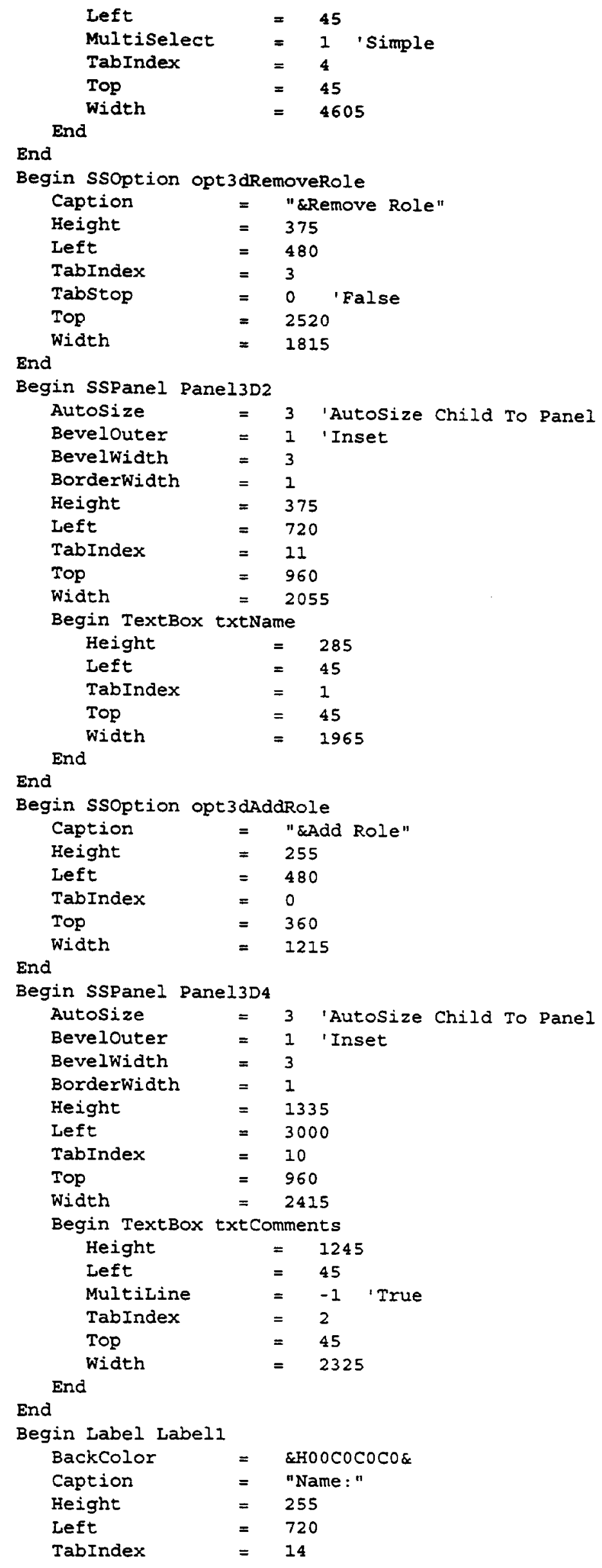




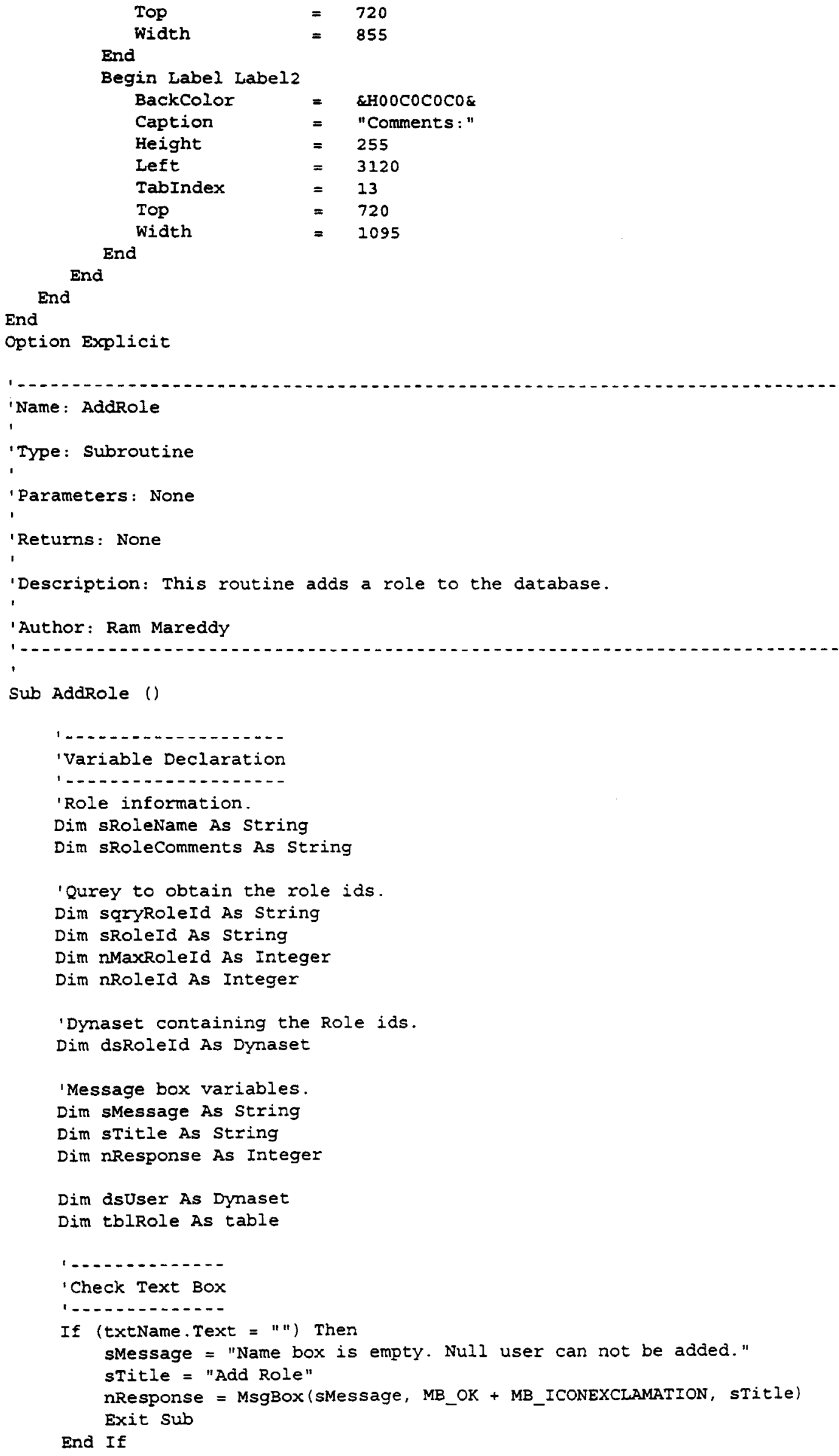




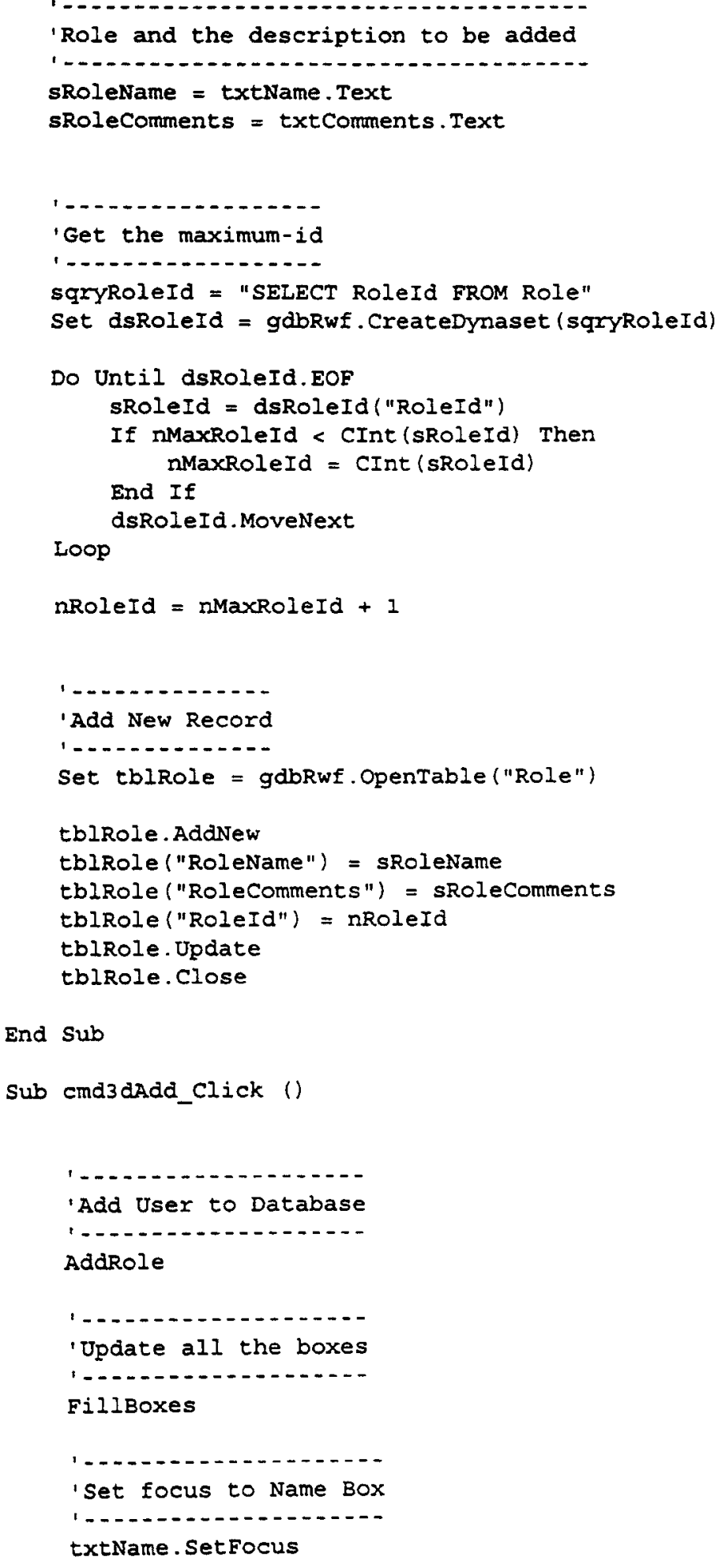




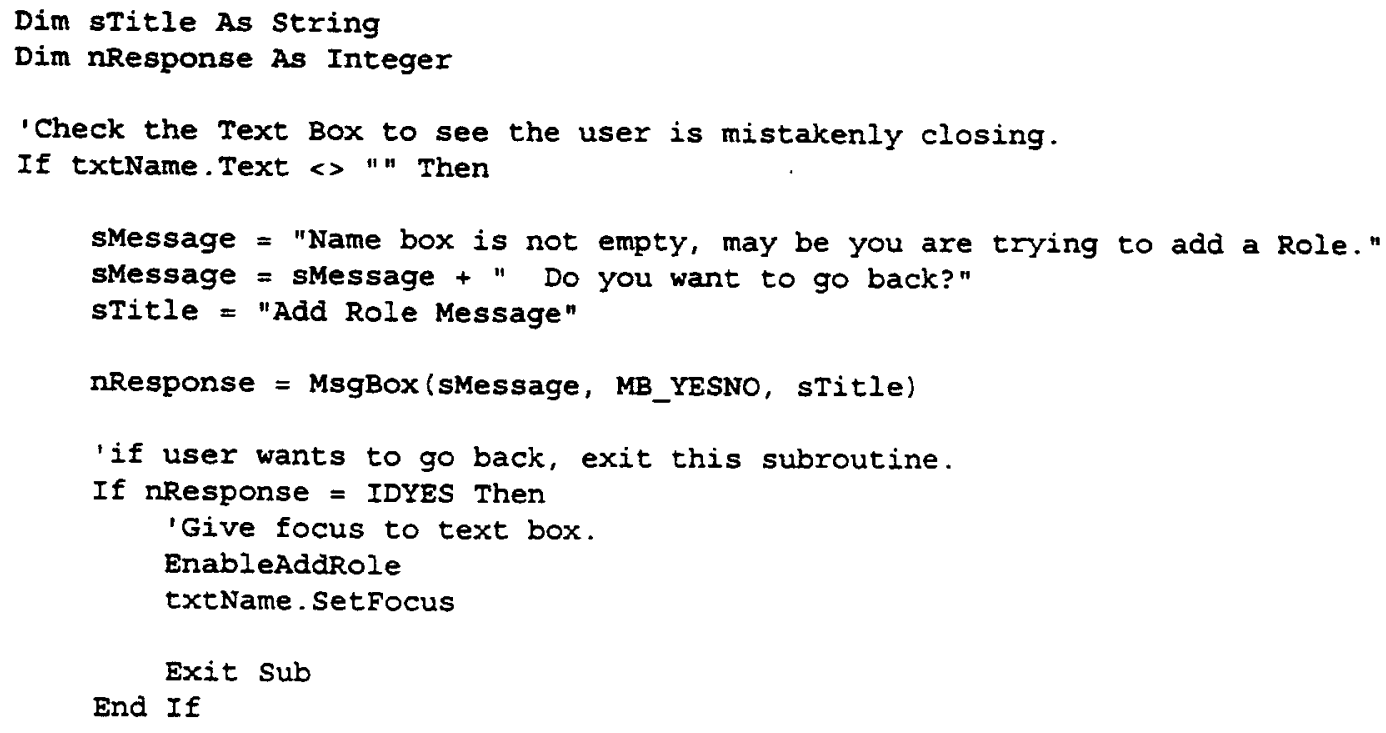

End If

Unload Me

End Sub

Sub cmd3dRemove_click ()

Removerole

End Sub

'Name: EnableAddRole

'Type: Subroutine

'Parameters: None

'Returns : None

'Description: This function enables all the add Role boxes and buttons, 'where as the EnableRemoverole does the vice versa.

'

Sub EnableAddRole ()

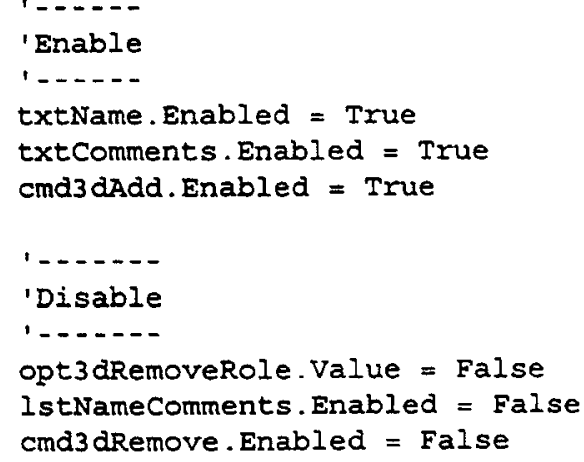

End sub 
'Fill Name+Description List Box

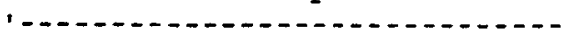

'First clear the list box.

IstNameComments. Clear

Do Until dsName.EOF

sNameDescription $="("+$ dsName ("RoleName" $)+") "$

sNameDescription $=$ sNameDescription $+\operatorname{Space}(20-$ Len $($ sNamedescription $))+": "$

sNameDescription = sNameDescription + " (" + dsName ("RoleComments") + ")"

IstNameComments.AddItem sNameDescription

dsName . MoveNext

Loop

End sub

Sub Form_Load ()

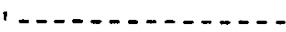

'Center the form

1...............

CenterForm Me

Fill boxes on the form with details

'

FillBoxes

End sub

Sub opt3dAddRole_click (Value As Integer)

EnableaddRole

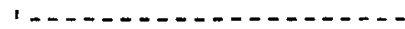

'Set focus to Name Box

1.-.-.-.....-.-......-

txtName.setFocus

End sub

Sub opt3dRemoverole_click (Value As Integer)

EnableRemoveRole

End Sub

Name: Removerole

'Type: Subroutine

'Parameters : None

'Returns : None

'Description: This function removes one or more users selected in the

'remove list box.

'Author: Ram Mareddy

' 1,

Sub Removerole () 


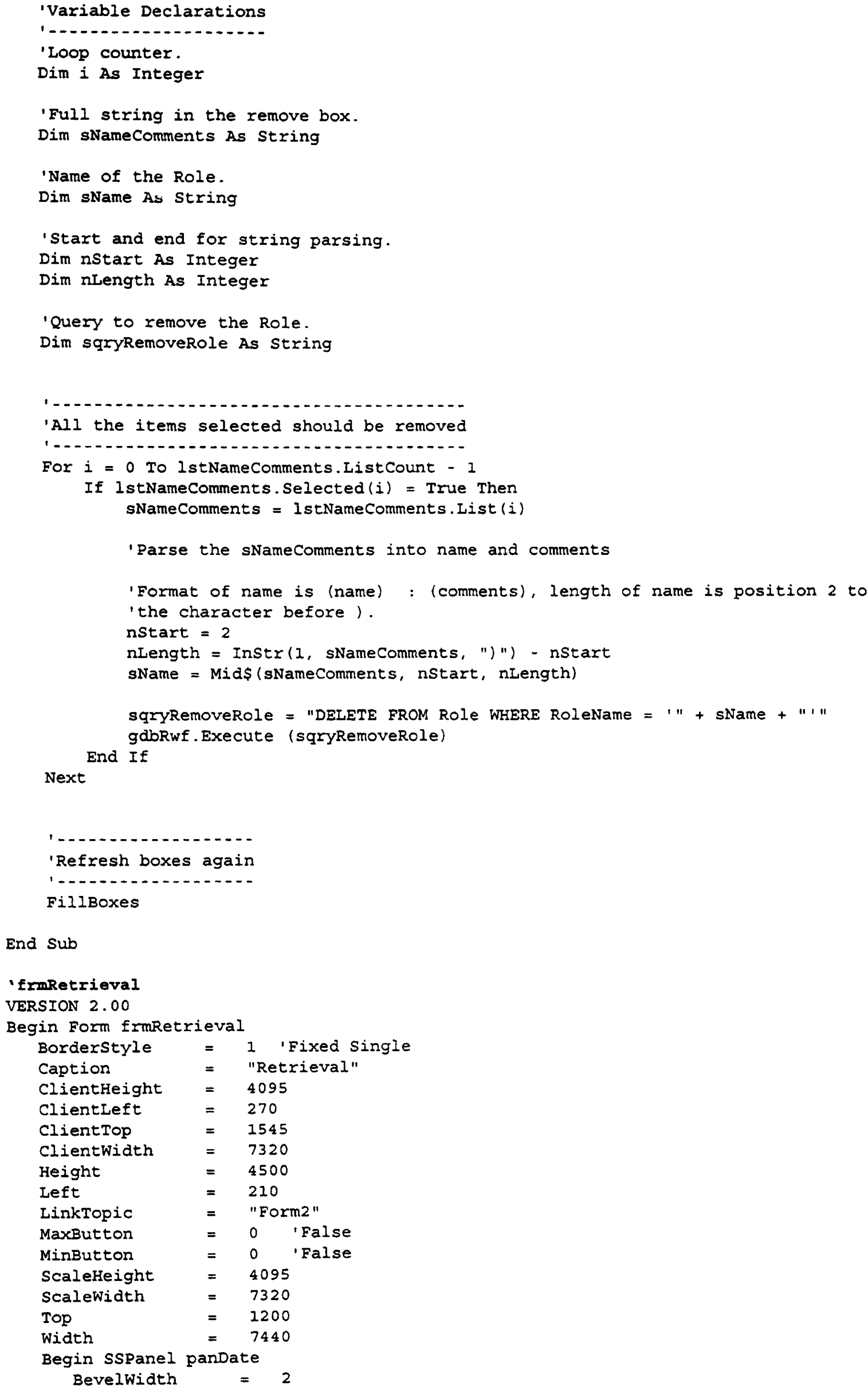

'Refresh boxes again

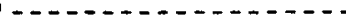

FillBoxes

End sub 


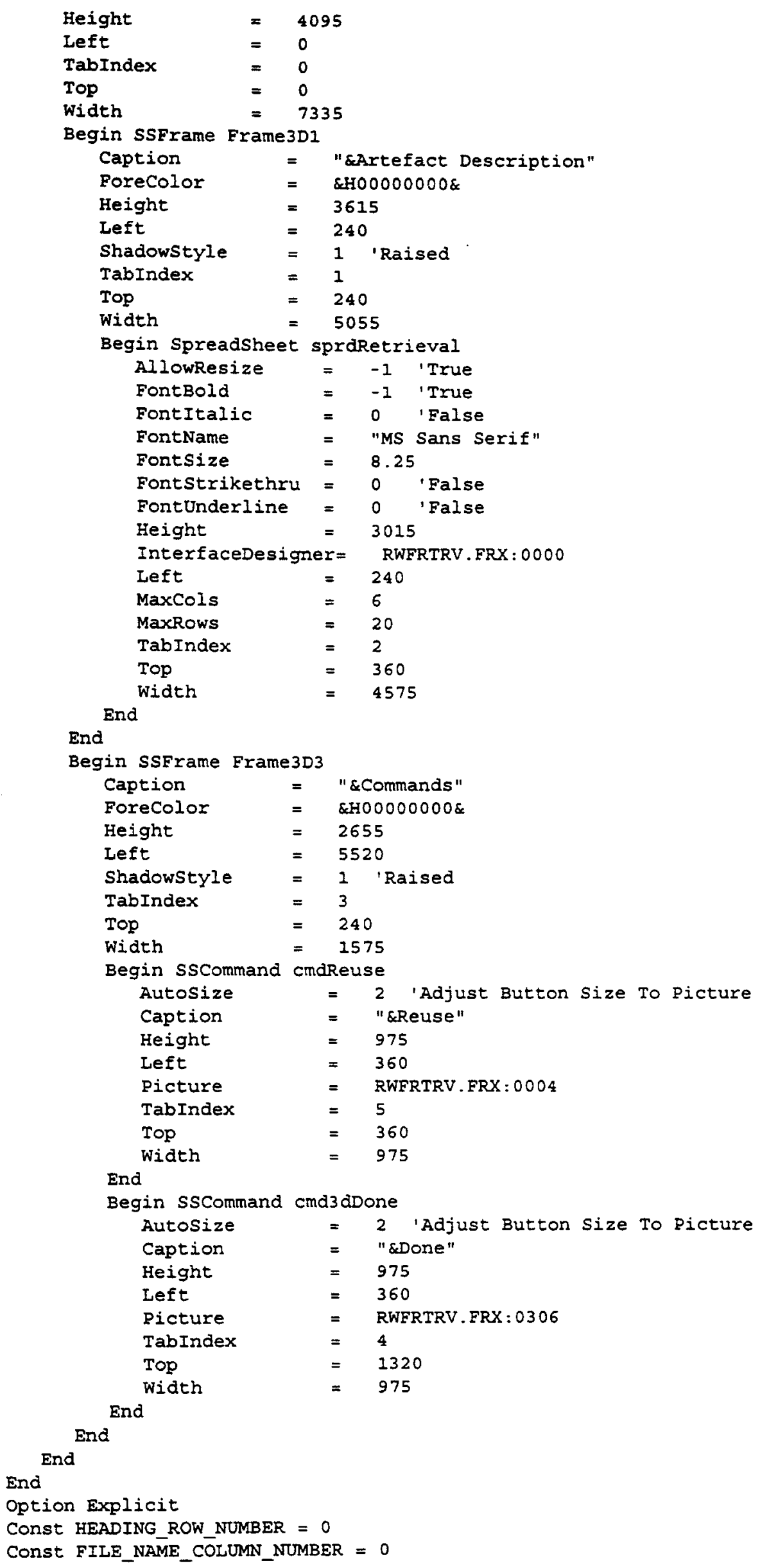




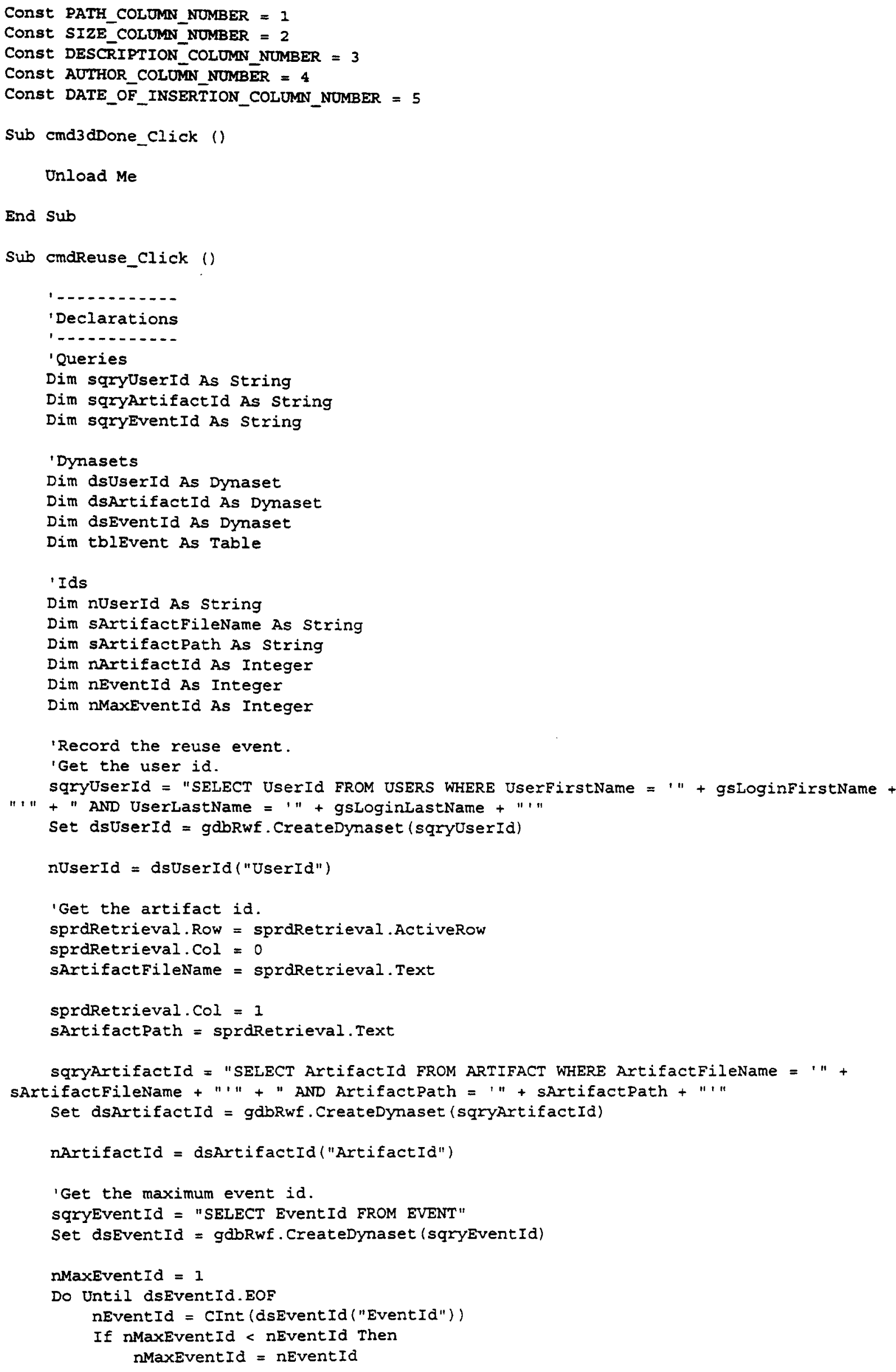




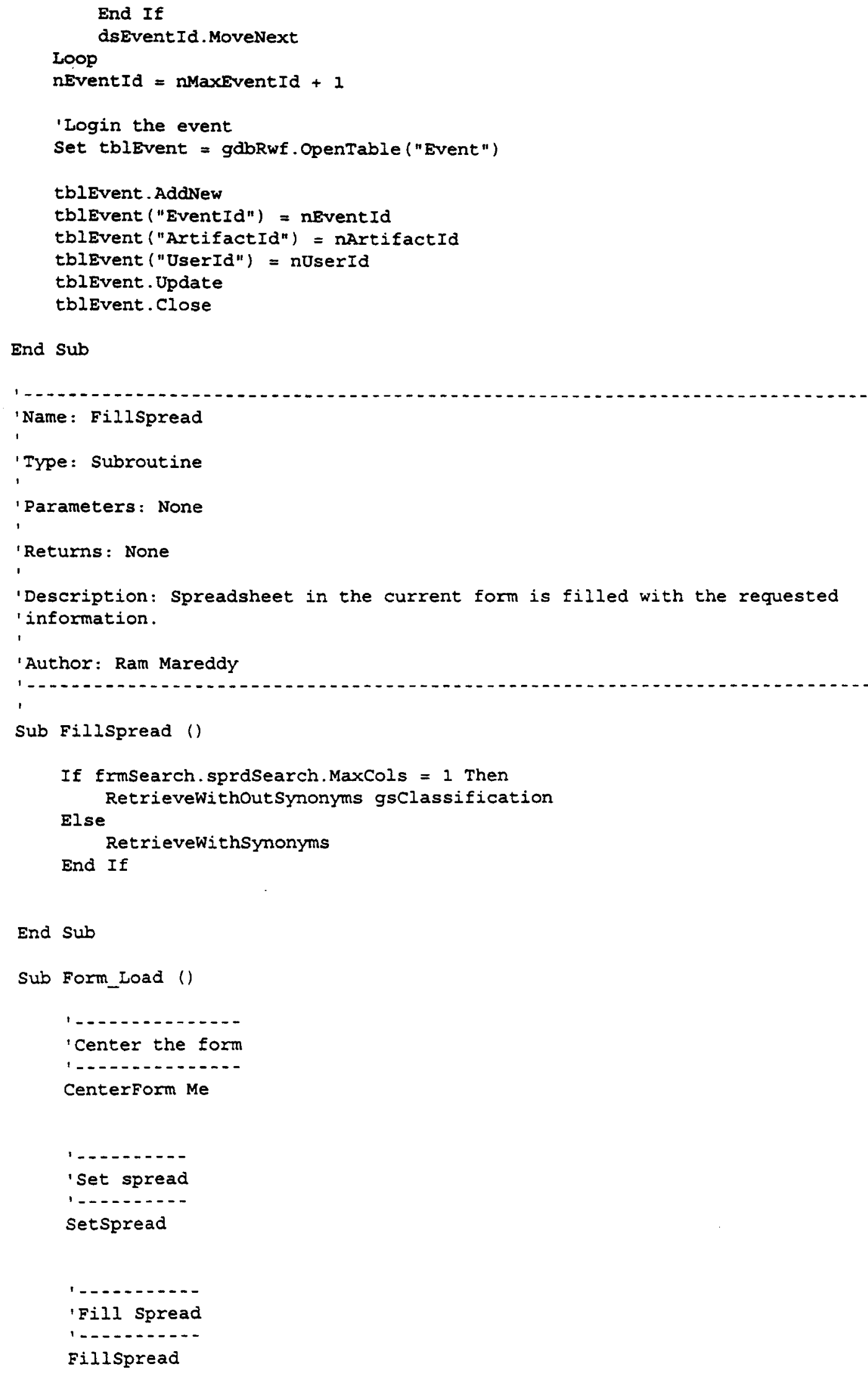

End Sub 


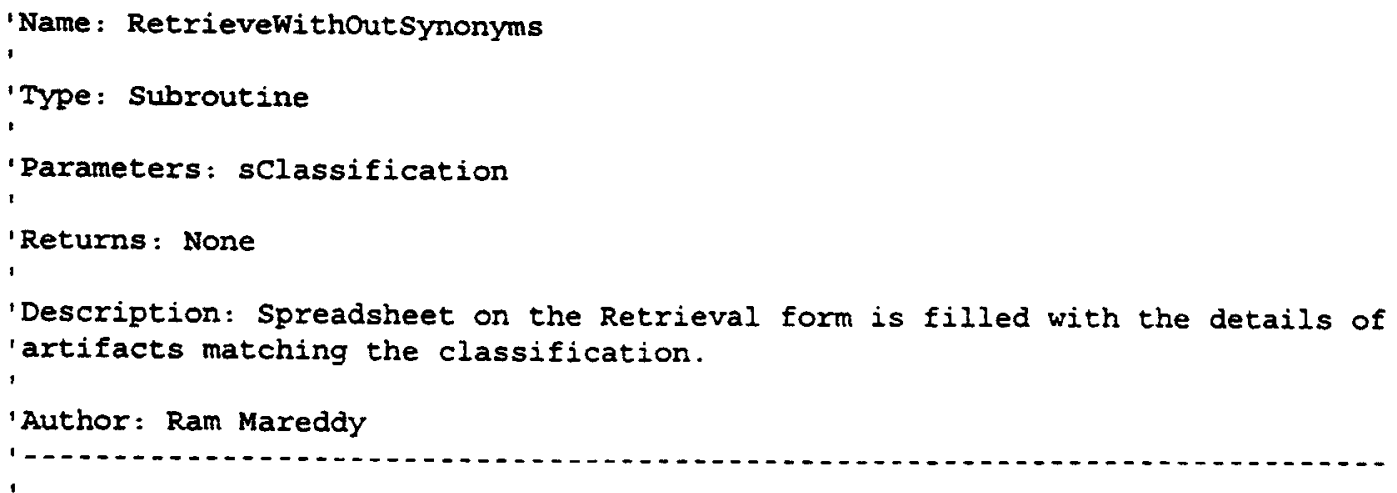




\section{Loop}

sprdRetrieval MaxRows $=$ ncurrentRow -1

End sub

'Name: Retrievewithsynonyms

'Type: subroutine

'Parameters: None

'Returns: None

'Description: Spreadsheet on the Retrieval form is filled with the details of 'artifacts obtained from synonym array.

'Author: Ram Mareddy

Sub Retrievewithsynonyms ()

'Variable Declarations
'String that holds the query for the vocabulary id.
Dim sqryclassification As String

'Dynaset that holds the results.

Dim dsclassification As Dynaset

'Row number for the current artifact.

Dim nCurrentRow As Integer

'Classification string of the current artifact.

Dim sCurrentartifact As string

Dim nCounter As Integer

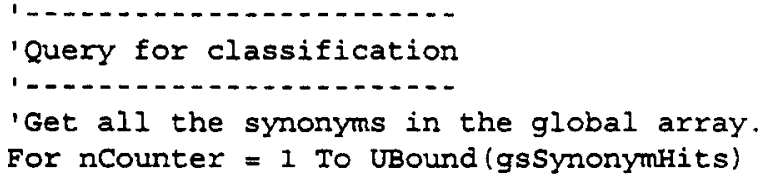

sqryClassification = "SELECT ArtifactFileName, ArtifactPath, ArtifactSize, ArtifactDescription, ArtifactAuthor, artifactDateofInsertion EROM ARTIFACT where VocabularyIdString $=1 "+$ gsSynonymits (nCounter) + " $1 "$

Set dsClassification $=$ gdbRwf. CreateDynaset (sqryClassification)

nCurrentRow $=1$

sprdRetrieval. Row $=$ nCurrentRow

sprdRetrieval.COl $=$ FILE_NAME_COLUMN_NUMBER

sprdRetrieval. Text $=$ dsclassification ("ArtifactFileName")

sprdRetrieval.COI = PATH_COLUMN_NUMBER

sprdRetrieval . Text = dsClassification ("ArtifactPath")

SprdRetrieval.COI $=$ SIZE_COLUMN_NUMBER

sprdRetrieval. Text = dsclassification ("Artifactsize")

sprdRetrieval.COl $=$ DESCRIPTION_COLUMN_NUMBER

sprdRetrieval. . Text = dsclassification ("ArtifactDescription") 


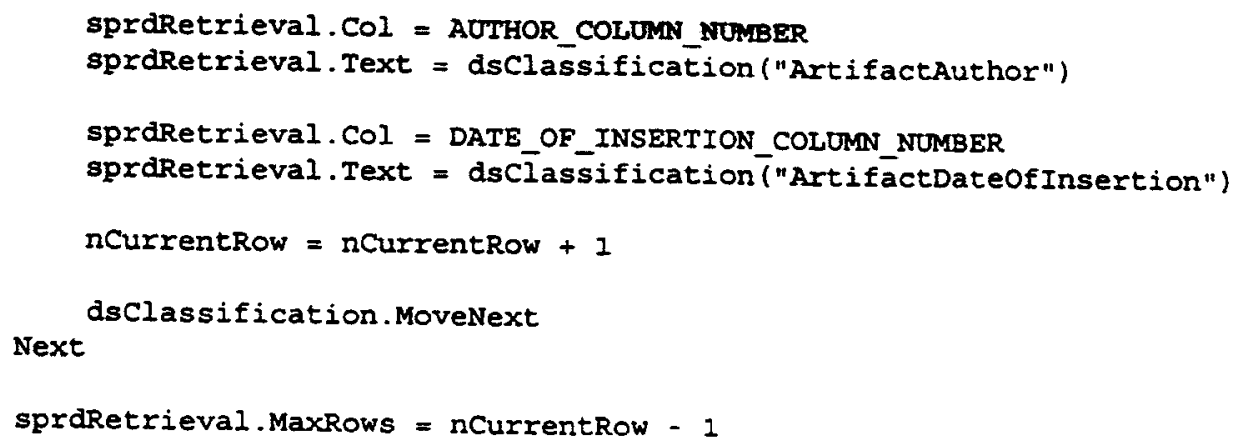

End sub

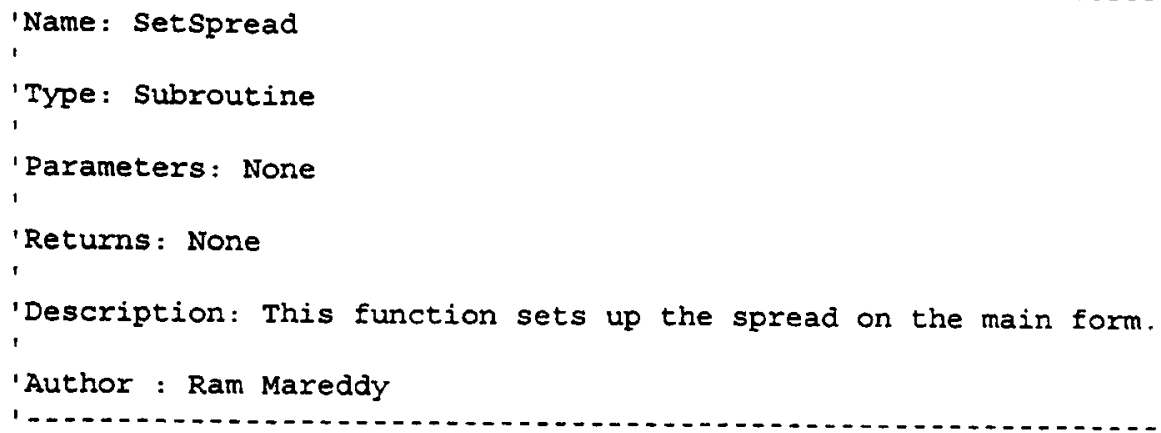


sprdRetrieval. FontSize = gudtRwfIni.nPontsize sprdRetrieval. FontName = gudtRwfIni. sFontName sprdRetrieval. FontBold $=$ gudtRwfIni.bFontBold

SpreadsetText sprdRetrieval, FILE_NAME_COLUMN_NUMBER, HEADING_ROW_NUMBER, "File" spreadsetText sprdRetrieval, PATH_COLUMN_NUMBER, HEADING_ROW_NUMBER, "Path" SpreadsetText sprdRetrieval, SIZE_COLUMN_NUMBER, HEADING_ROW_NUMBER, "Size" spreadsetText sprdRetrieval, DESCRIPTION COLUMN NOMBER, HEADING ROW NOMBER, "Description"

SpreadsetText sprdRetrieval, AUTHOR COLOMN NUMBER, HEADING ROW NOMBER, "Author" SpreadsetText sprdRetrieval, DATE_OF_INSERTION_COLUMN_NUMBER, HEAOING_ROW_NOMBER, "Date of Insertion"

End Sub

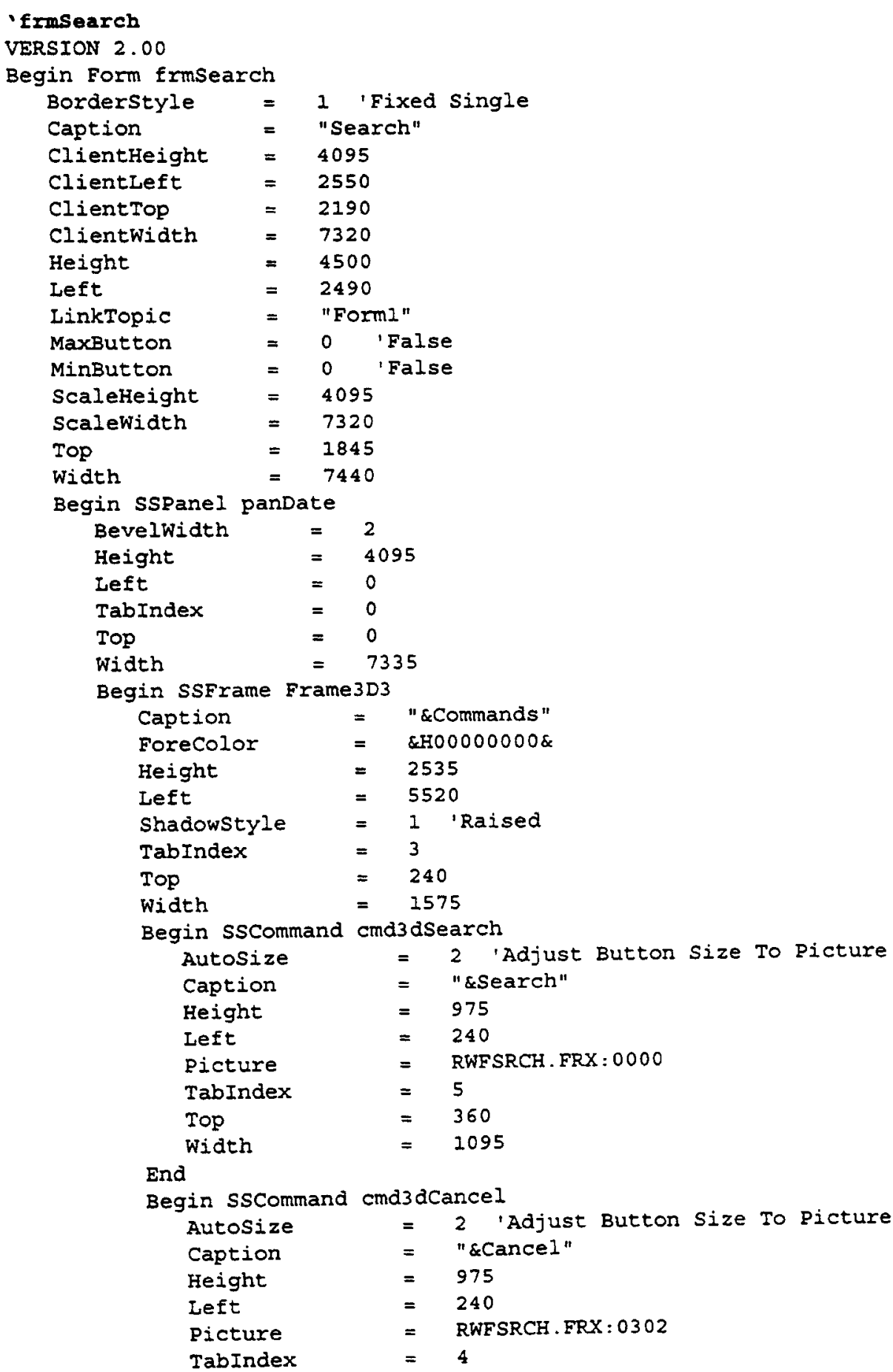




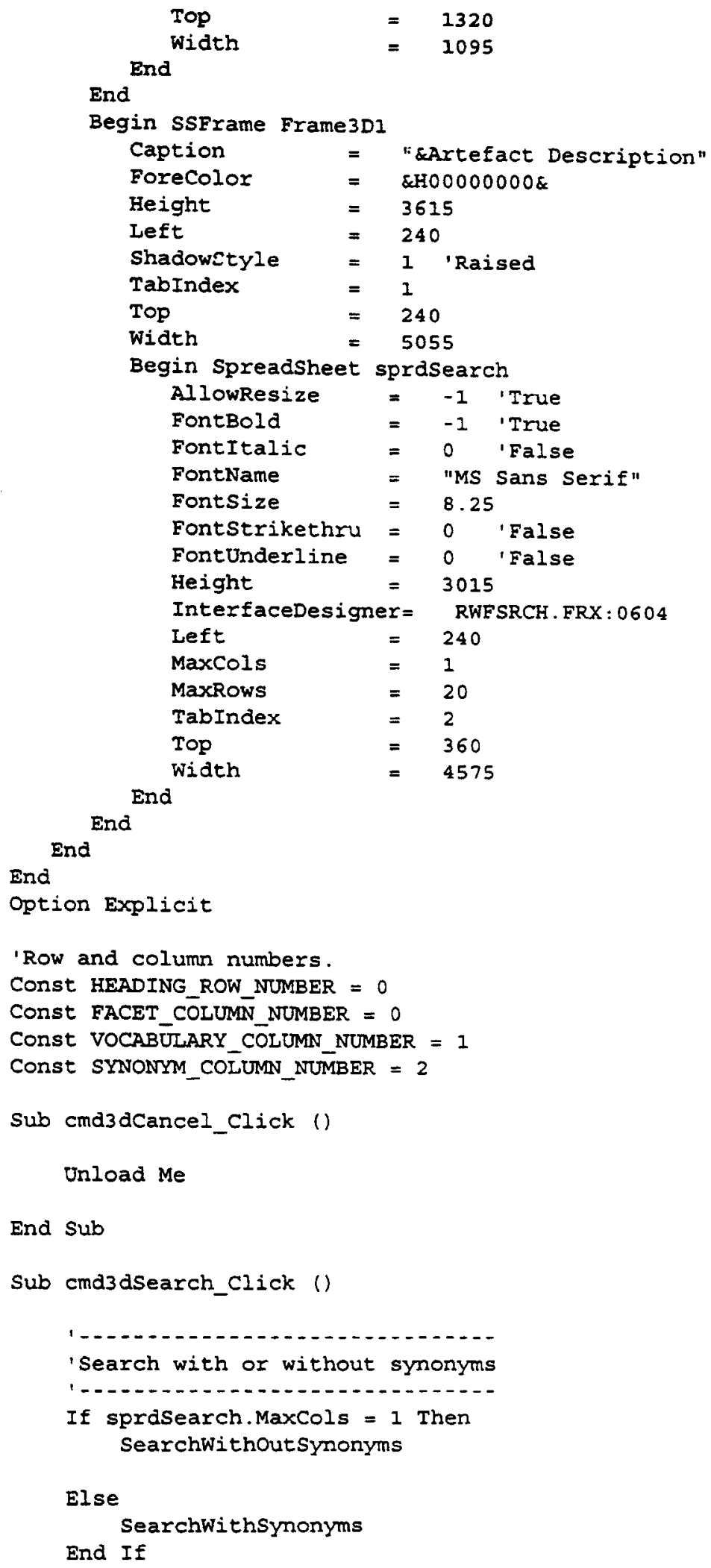




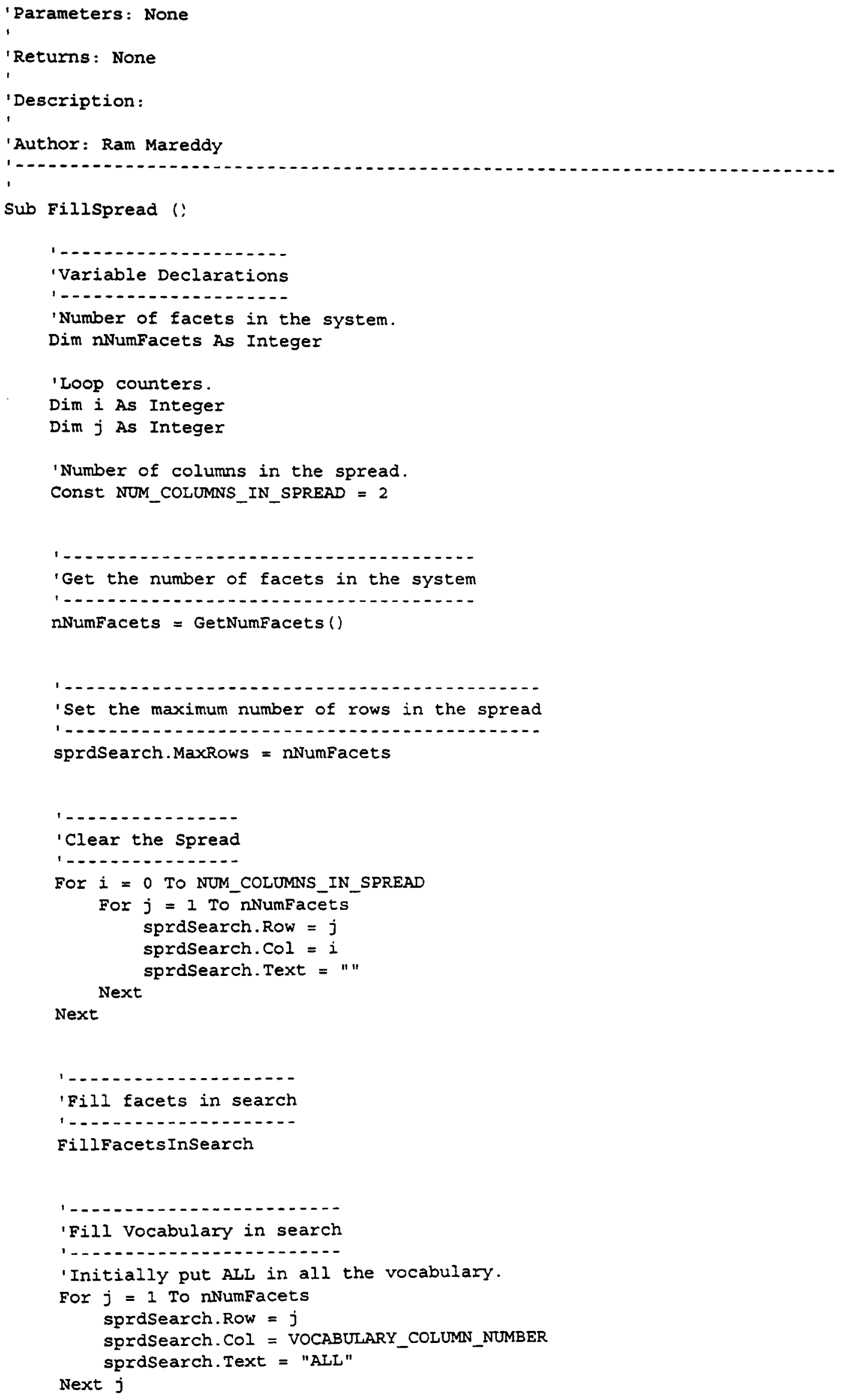

End Sub 
Sub Form_Load ()

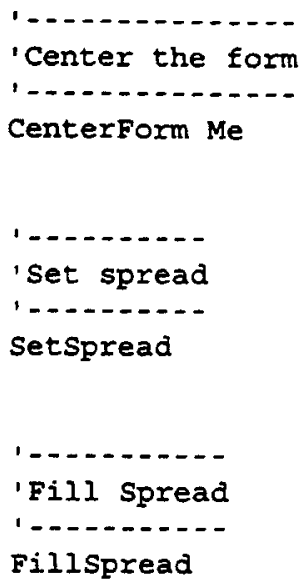

End Sub

\section{'Name: GetClassificationstring}

'Type: Subroutine

'Parameters: sclassification

'Returns: None

'Description: Gets the classification for the currently requested artifacts.

'Author: Ram Mareddy

$1-$

Sub Getclassificationstring (sclassification As string)

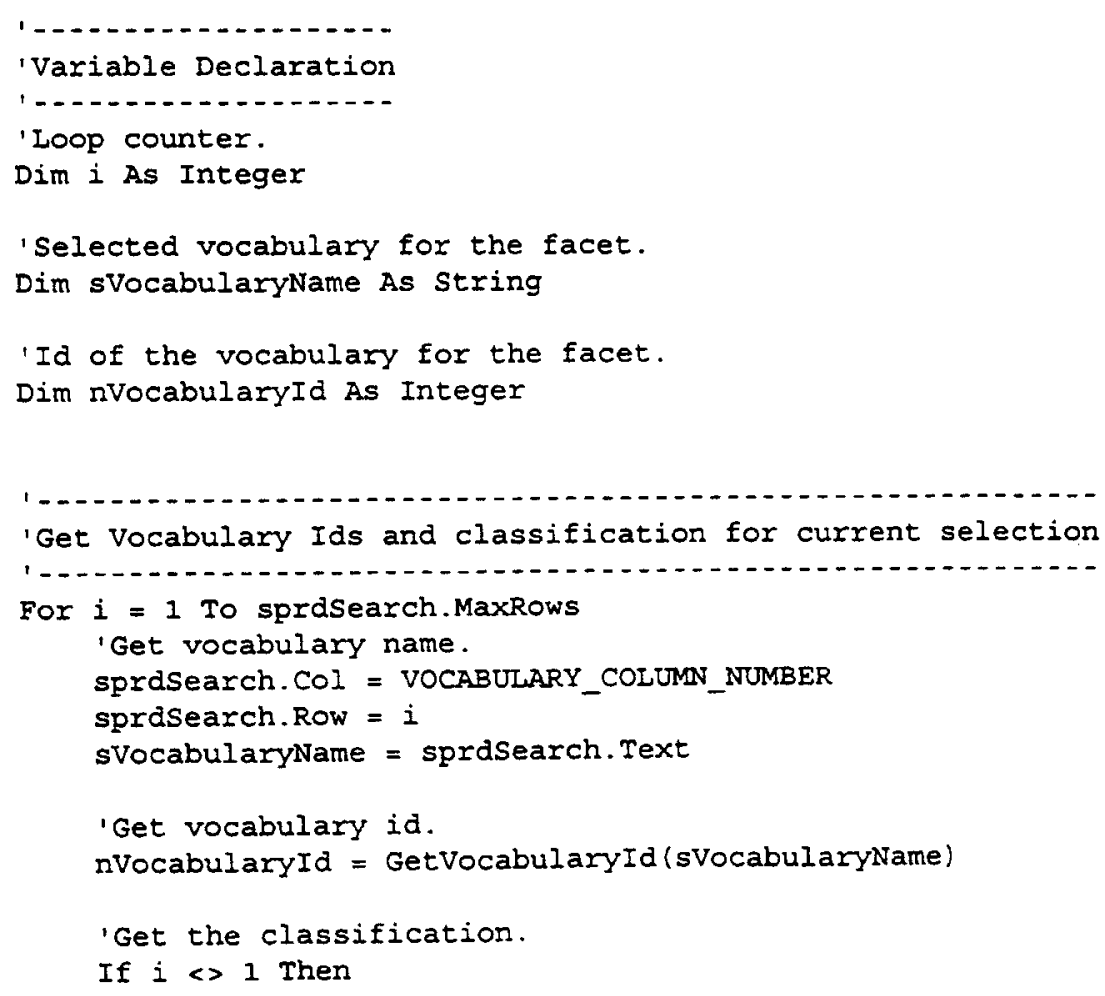




$$
\text { Else }
$$

Next $i$

End $s u b$

' Name: GetSynonymflagstring

'Type: Subroutine

'Parameters: sSynonymflagstring

'Returns: None

'Description: The facets that are okd for the use of synonyms are returned 'in the form a string.

'Author: Ram Mareddy

1 Auchor: Ram Mareddy

Sub GetsynonymFlagstring (ssymonymFlagstring As String)

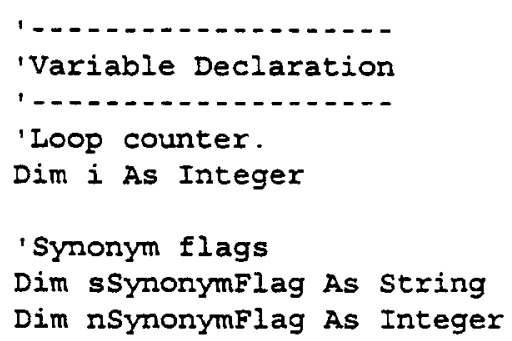

Next $i$

End Sub

Sub PresentArtifacts ()

frmRetrieval. Show Modal 
End Sub

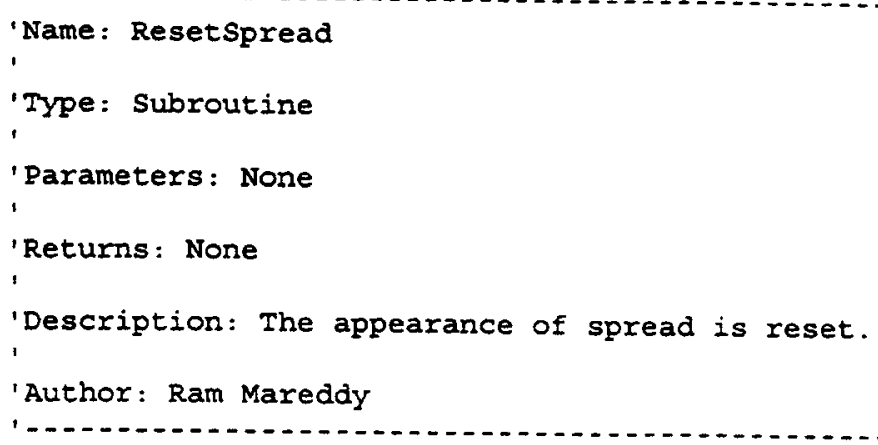

'Fill the synonym column with NOs 
For $i=1$ To sprdSearch. MaxRows

sprdSearch. Row $=i$

sprdSearch.COI $=$ SYNONYM_COLUMN_NOMBER

Next $i$

sprdsearch. Text $=$ "NO"

End Sub

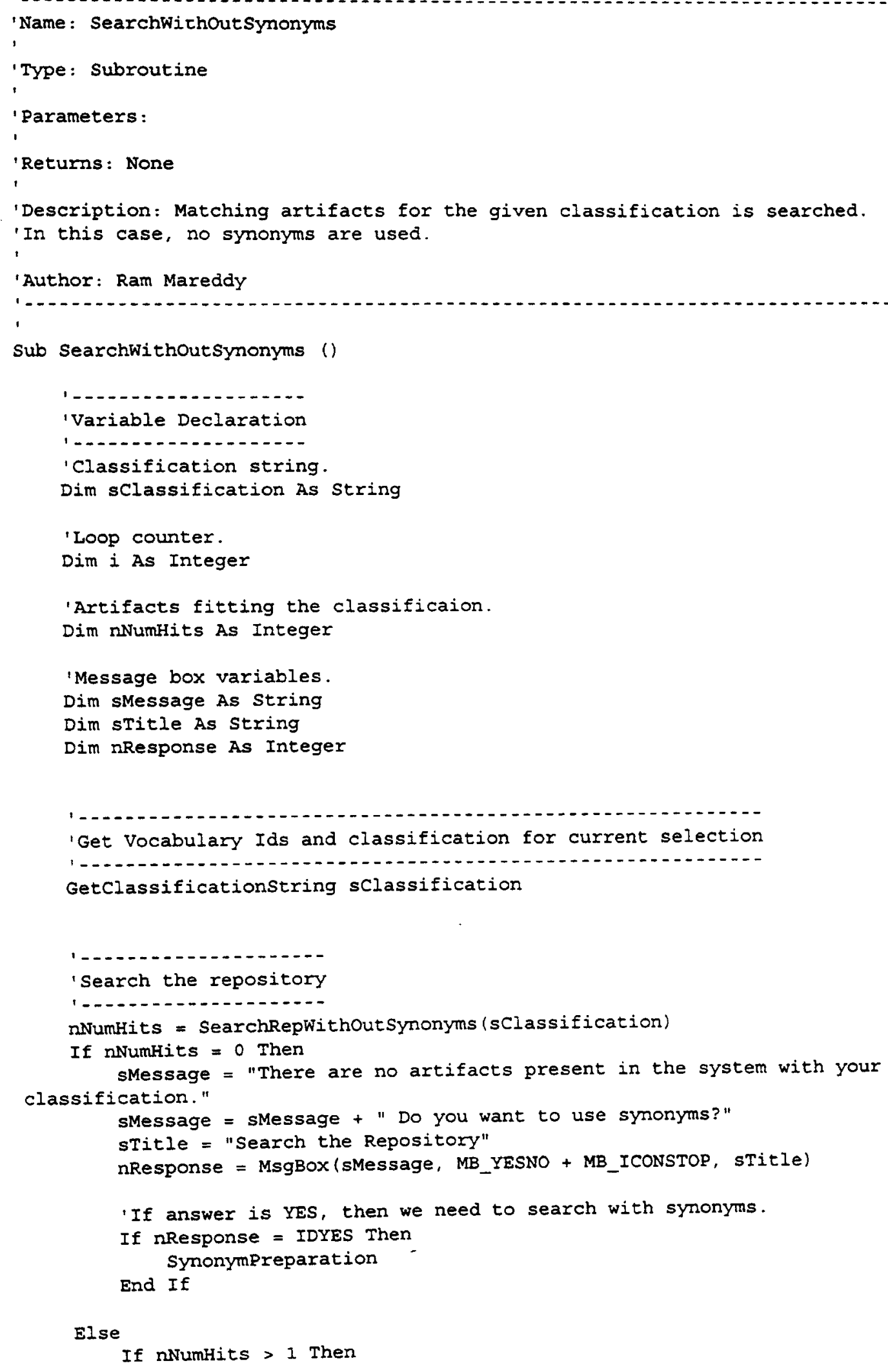




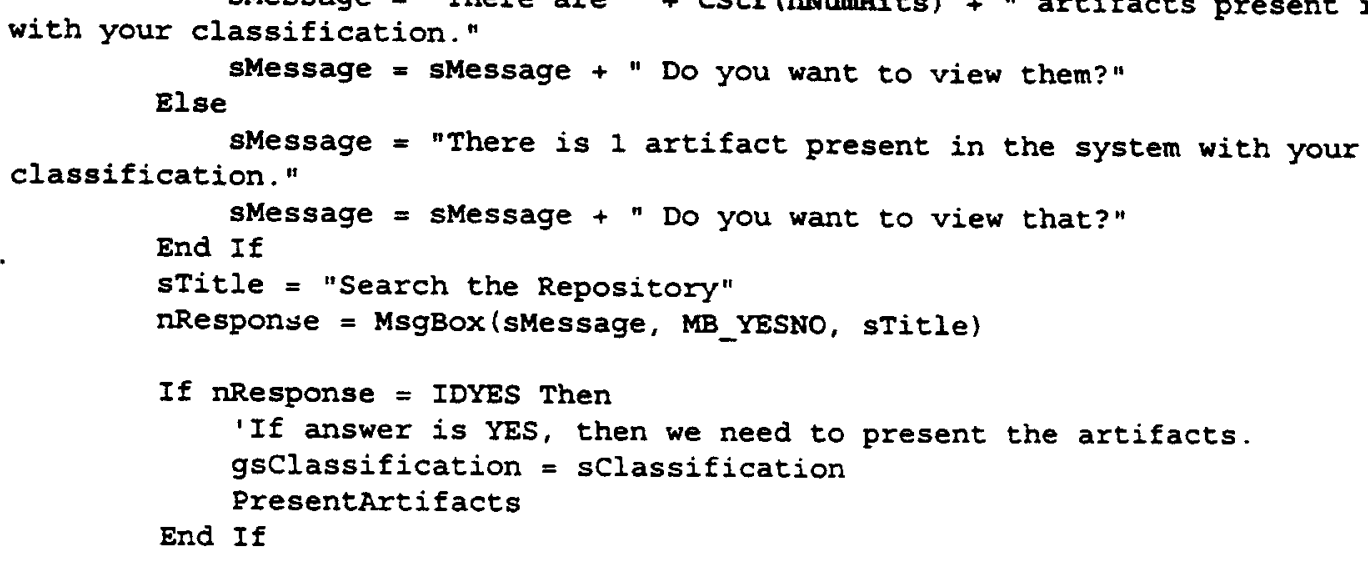

End If

End sub

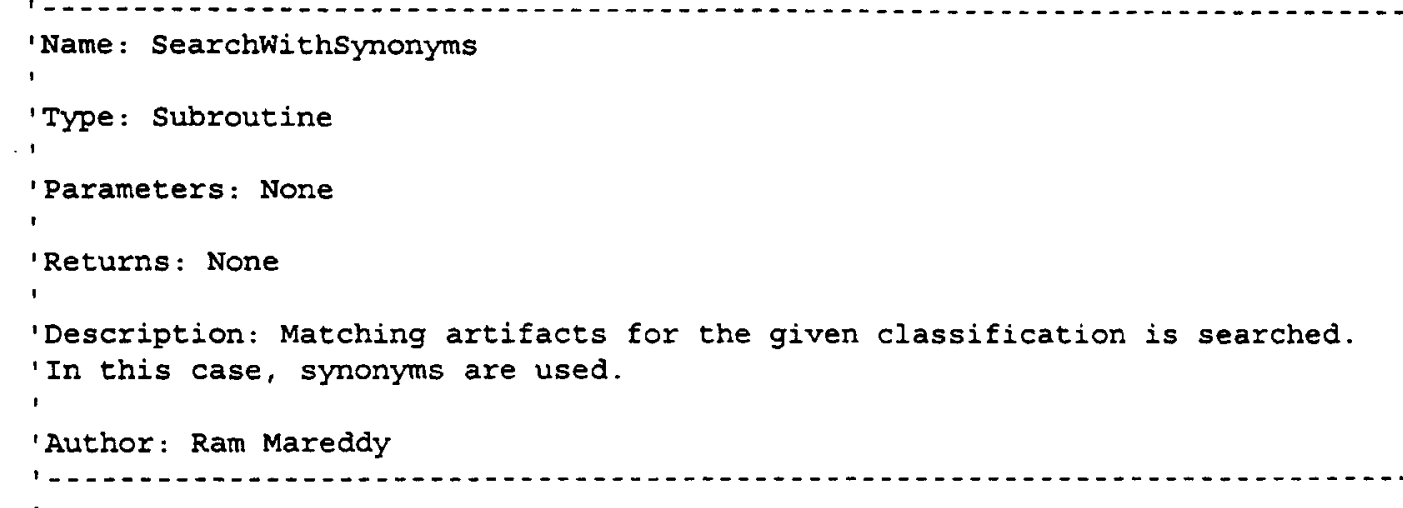




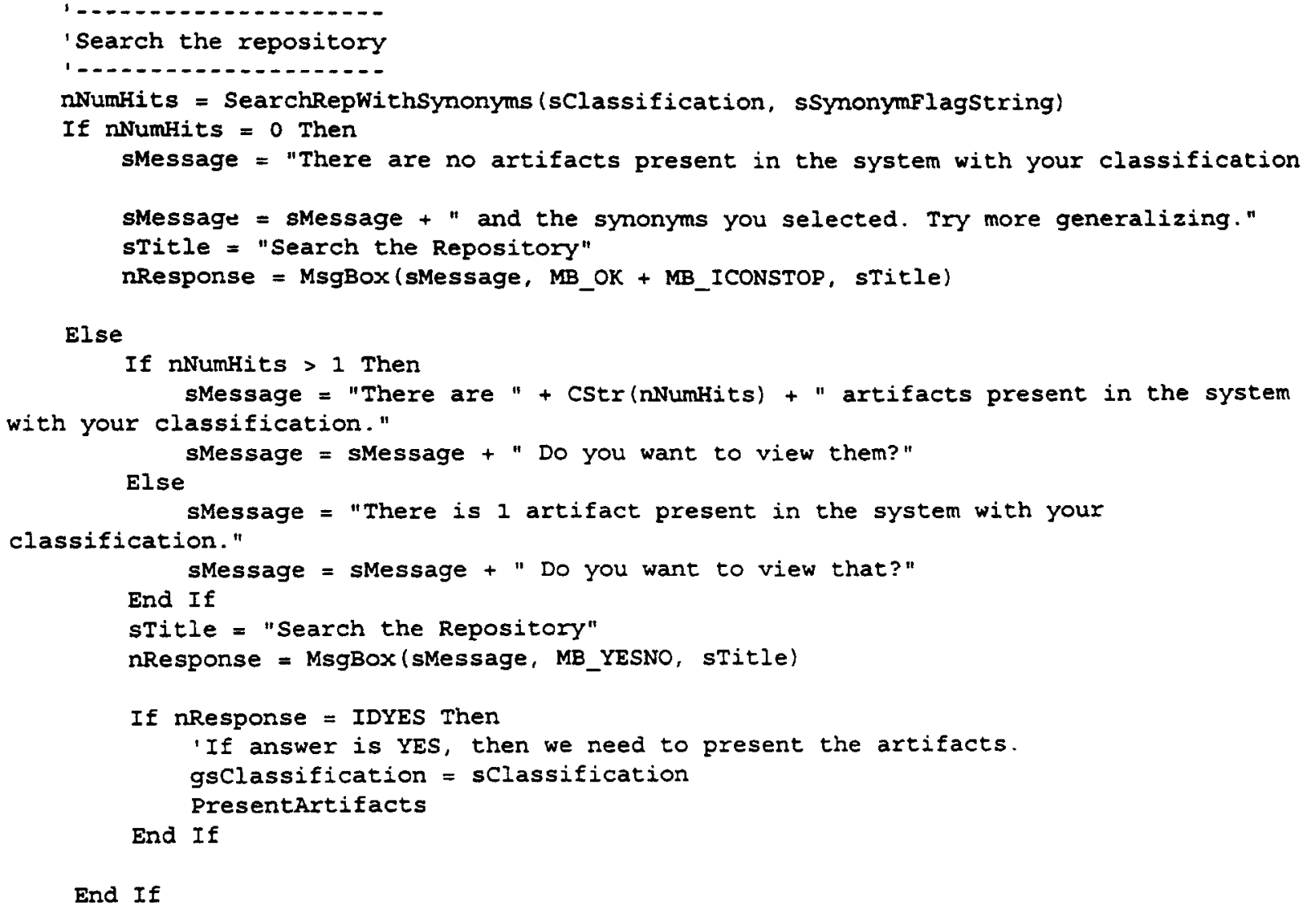

End Sub

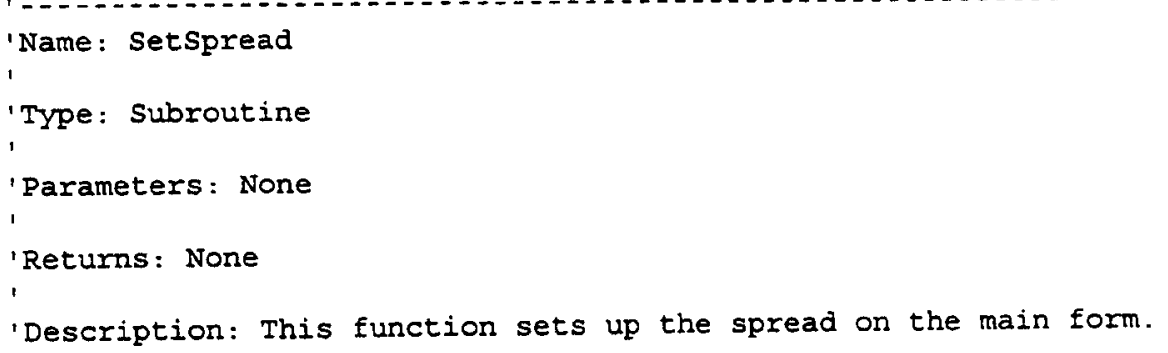




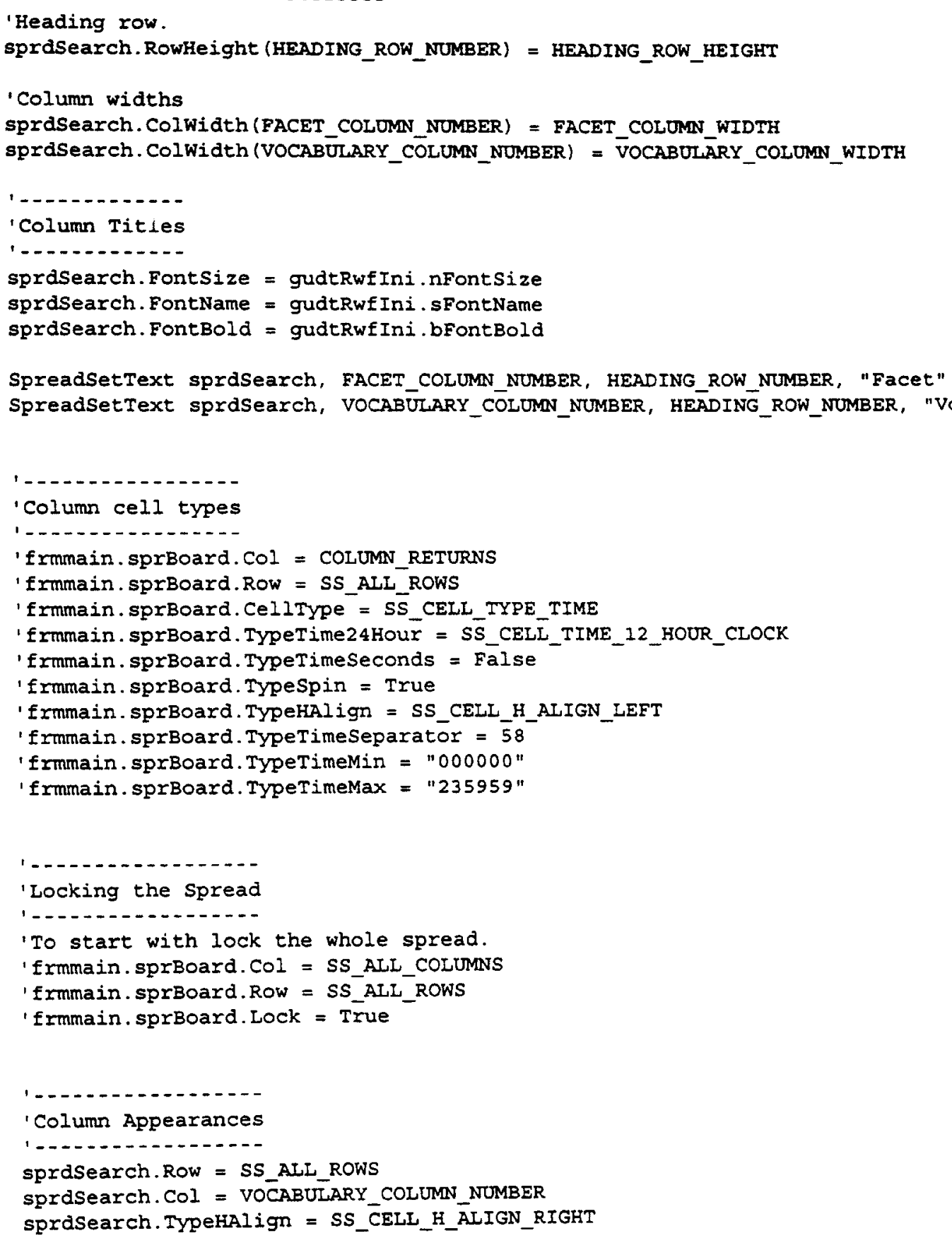

End Sub

'Name: spraSearch_click

Type: Subroutine

' Parameters: Col is the column of the selected cell, Row is the row of the 'selected cell. 
Sub sprasearch_click (Col As Long, Row As Long)

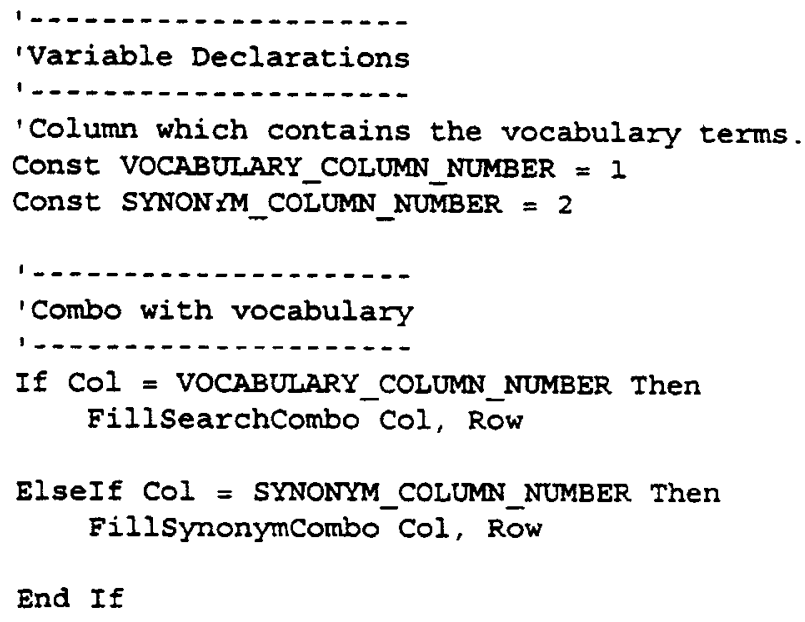

End Sub

Sub symonympreparation () 
ResetSpread

' Change the title of the search box

' Change the title of the search box

frmsearch.Caption $=$ "Search With Symonyms"

End Sub

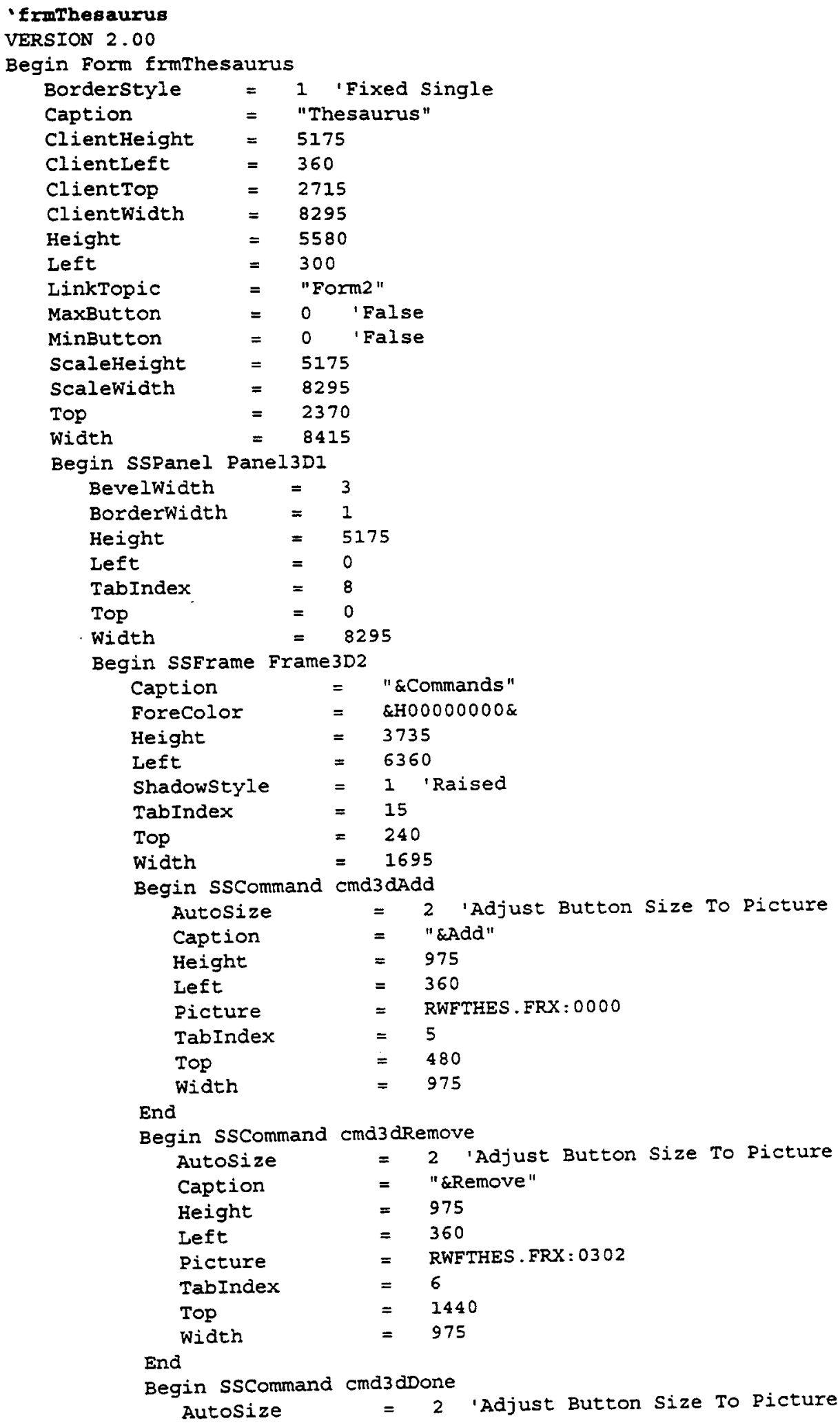




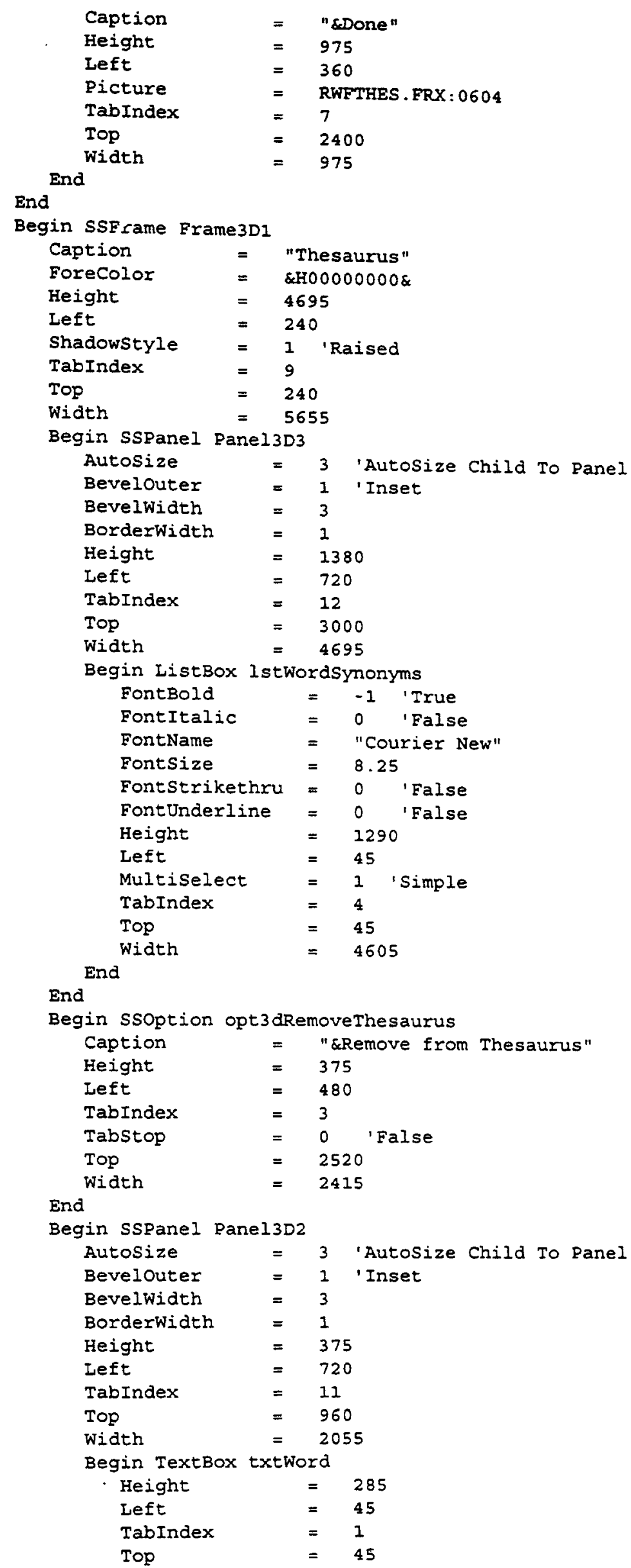




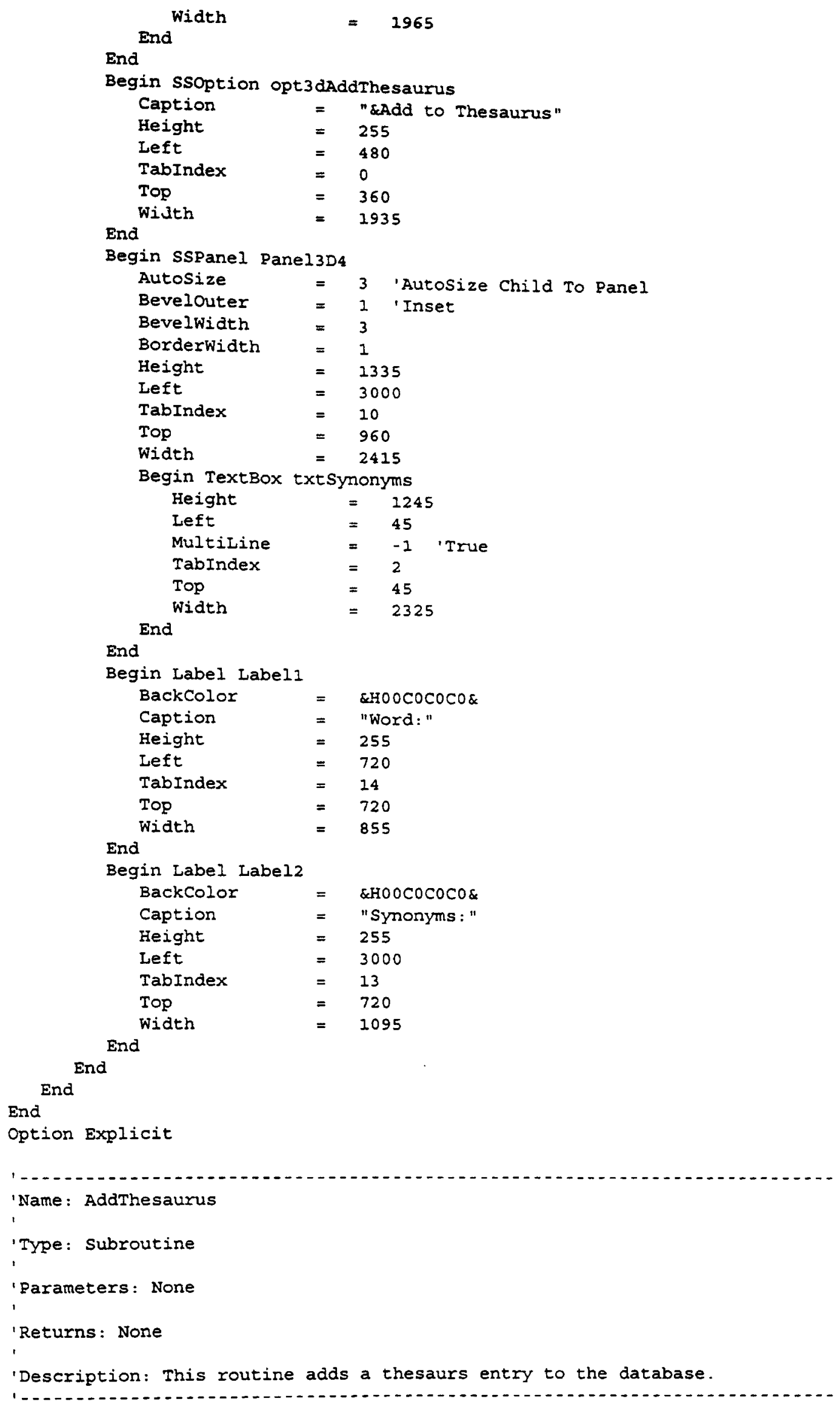

Sub AddThesaurus () 


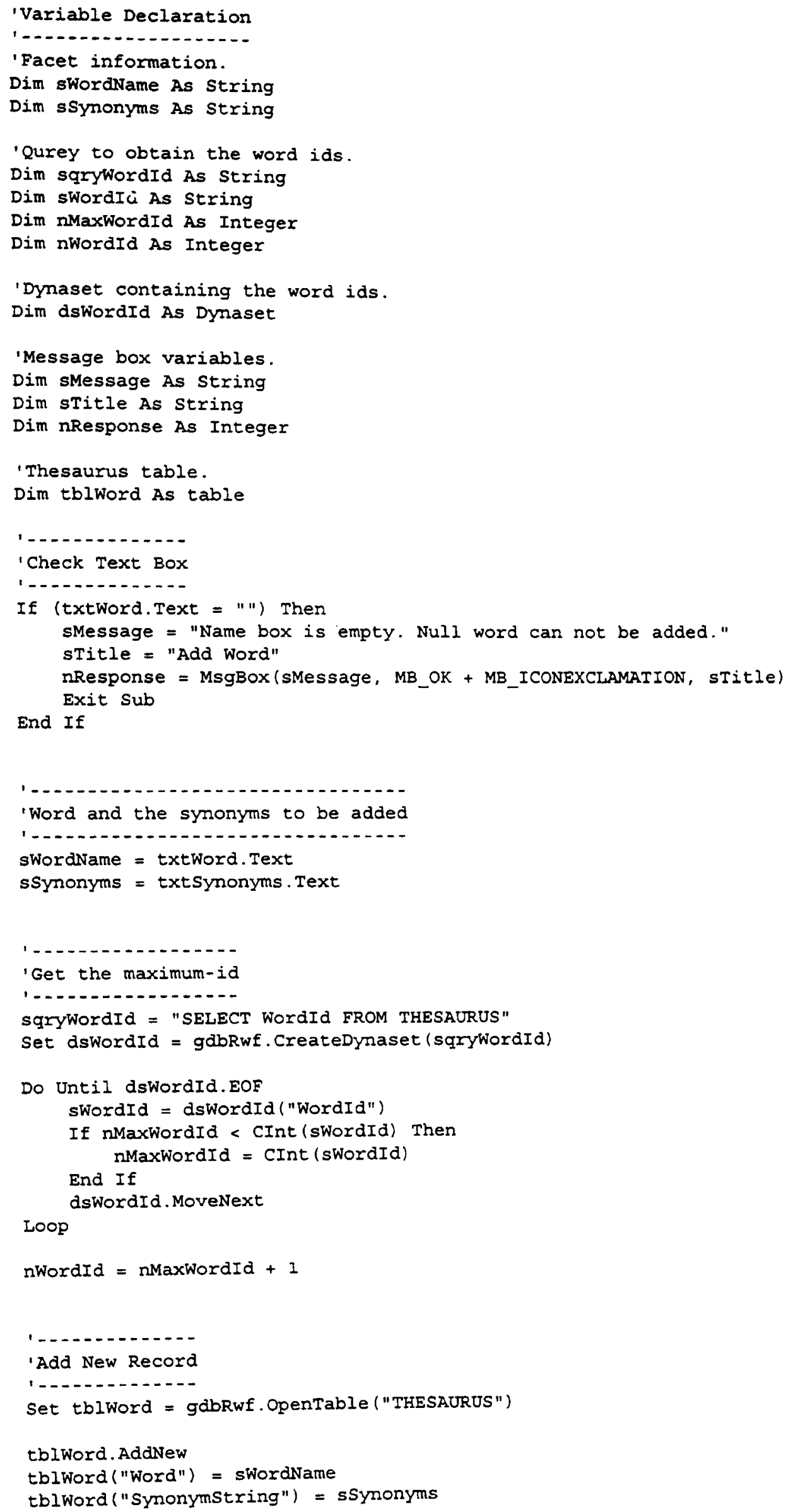


End Sub

'Name: EnableAddThesaurus

Type: subroutine

'Parameters: None

Returns: None

'Description: This function enables all the add thesaurus boxes and buttons, 'where as the EnableRemoveThesaurus does the vice versa.

'

Sub EnableAddThesaurus ()

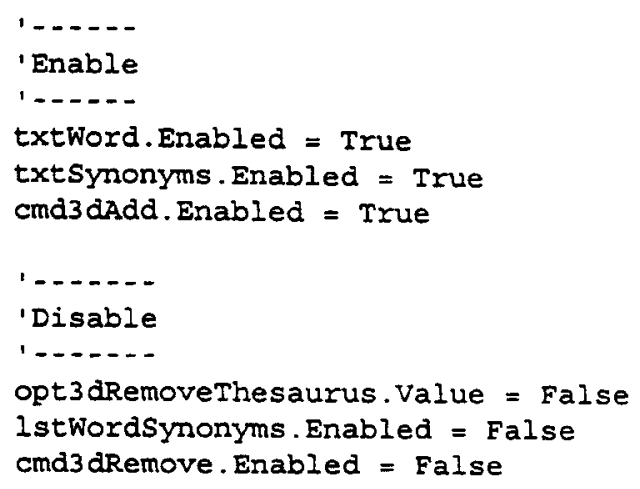

End Sub

'Name: EnableRemoveThesaurus

'Type: Subroutine

' Parameters: None

End $S u b$

'Name: FillBoxes 
'Type: Subroutine

'Parameters: None

'Returns: None

'

'Description: This subroutine fills all the boxes, i.e. text box, listbox

'with information. This can also be called after ADD or REMOVE is done.

'Author: Ram Mareddy

1

Sub FillBoxes ()

Dim sqryword As string

Dim swordsynonyms As string

Dim dsword As Dynaset

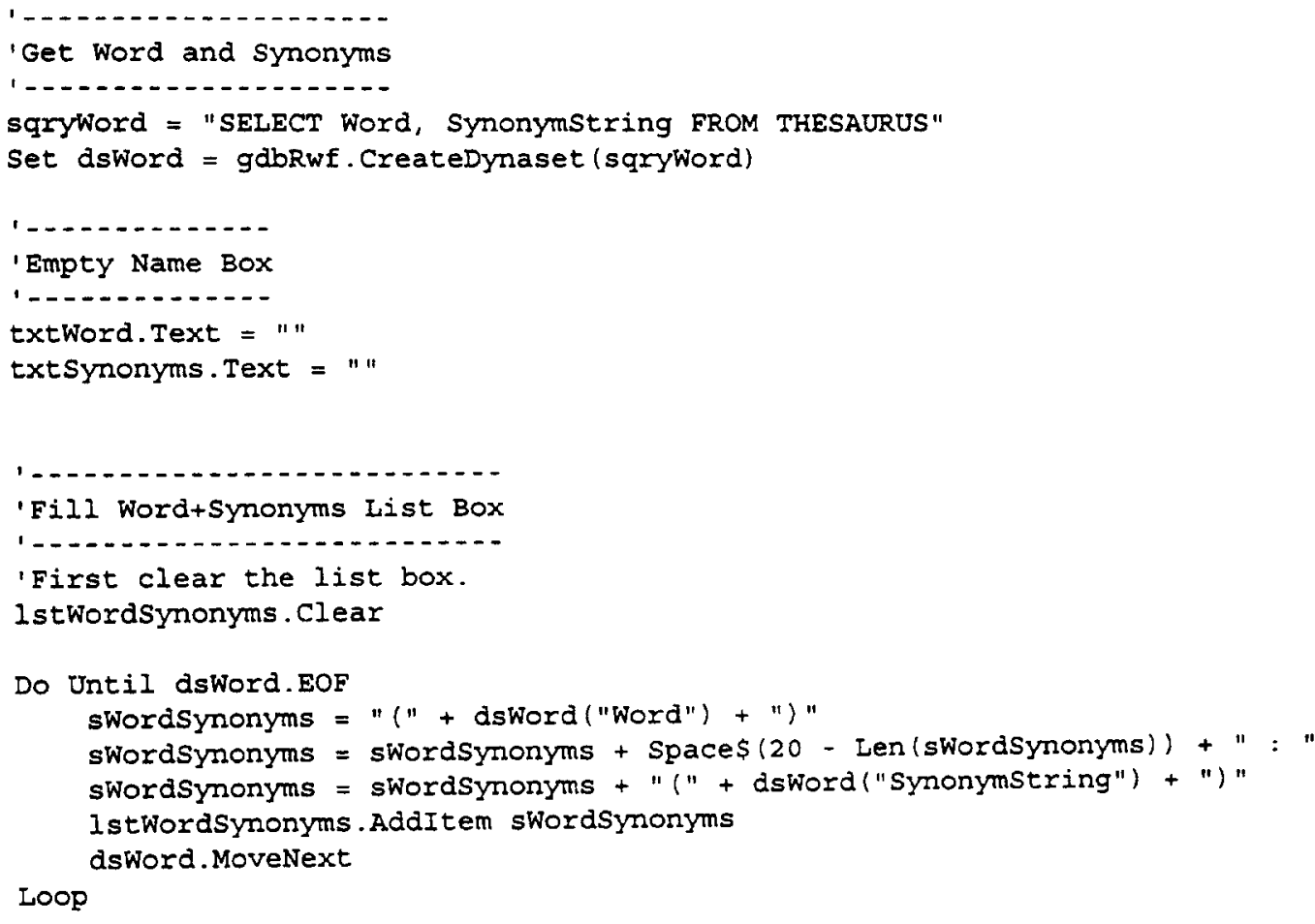

End sub

sub Form_Load ()

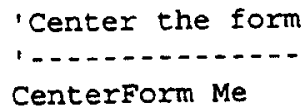

End sub

Sub opt3dadaThesaurus_Click (Value As Integer)

EnableAddThesaurus 
'Set focus to Name Box

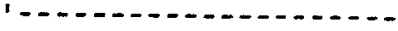

txtword. SetFocus

End sub

Sub opt3dRemoveThesaurus_Click (Value As Integer)

EnableRemoveThesaurus

End Sub

'Name: Removeword

Type: Subroutine

'Parameters: None

'Returns: None

'Description: This function removes one or more words selected in the 'remove list box.

'Author: Ram Mareddy

Sub Removeword ()

$------$

' Variable Declarations

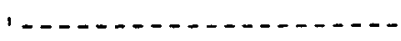

'Loop counter.

Dim i As Integer

'Full string in the remove box.

Dim swordSynonyms As string

'Name of the word.

Dim sword As string

'start and end for string parsing.

Dim nstart As Integer

Dim nlength As Integer

'Query to remove the word.

Dim sqryRemoveword As string

'All the items selected should be removed

For $i=0$ To lstwordsynonyms. Listcount - 1

For $i=0$ To lstwordsynonyms.Listcount -1

sWordSynonyms = lstWordSynonyms. List $(i)$

'Parse the swordsynonyms into word and synonyms

'Format of name is (word) : (synonyms), length of name is position 2 to 'the character before).

nstart $=2$

nLength = Instr(1, sWordsynonyms, ")") - nstart

sword = Mid\$ (swordsynonyms, nstart, nLength) 
Next

SqryRemoveWord $=$ "DELETE FROM THESAUROS WHERE WOYd $=\cdots+$ sWord $+"$ " gdbRwf.Execute (sqryRemoveword)

End If

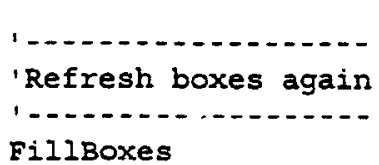

End sub

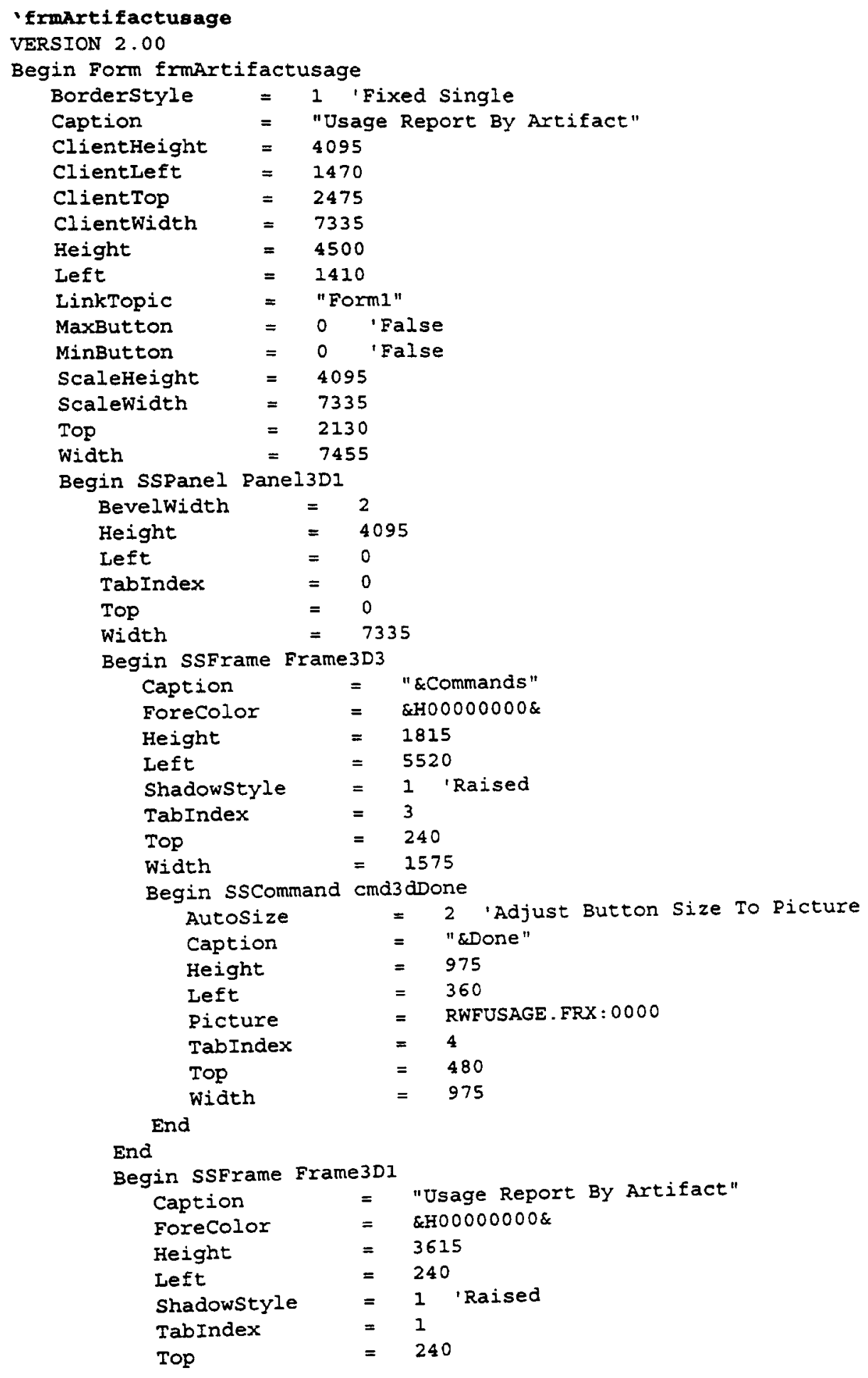




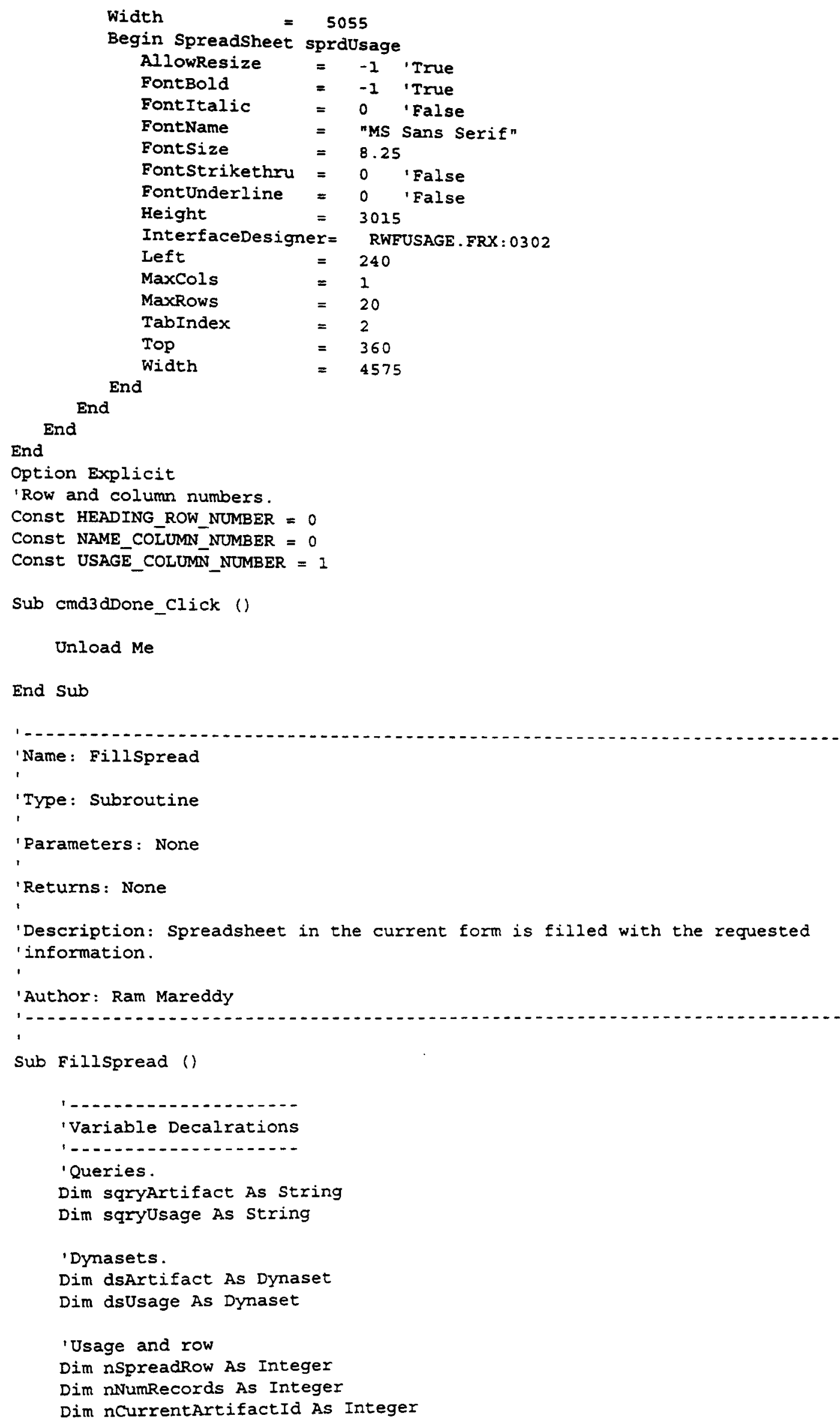




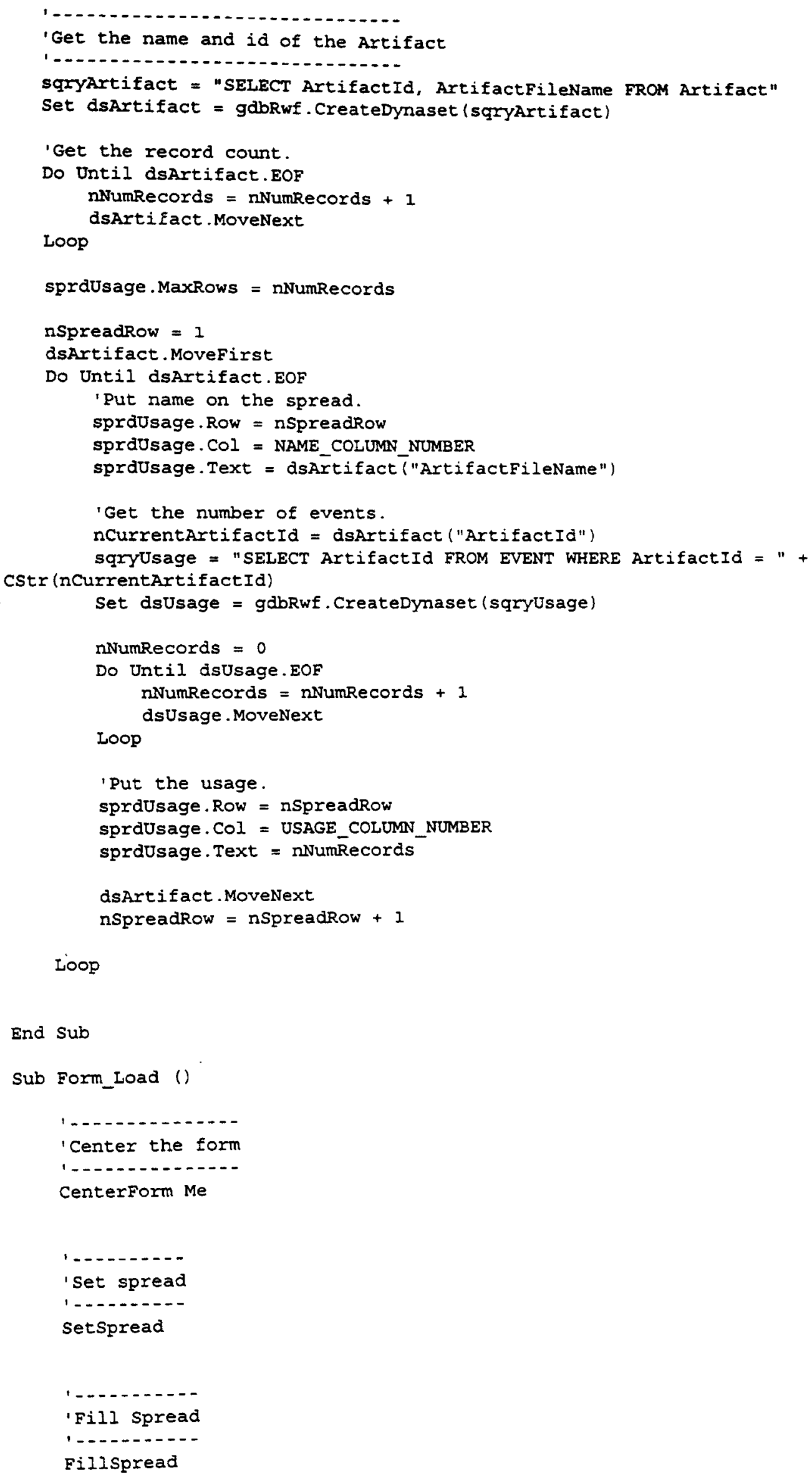


End Sub

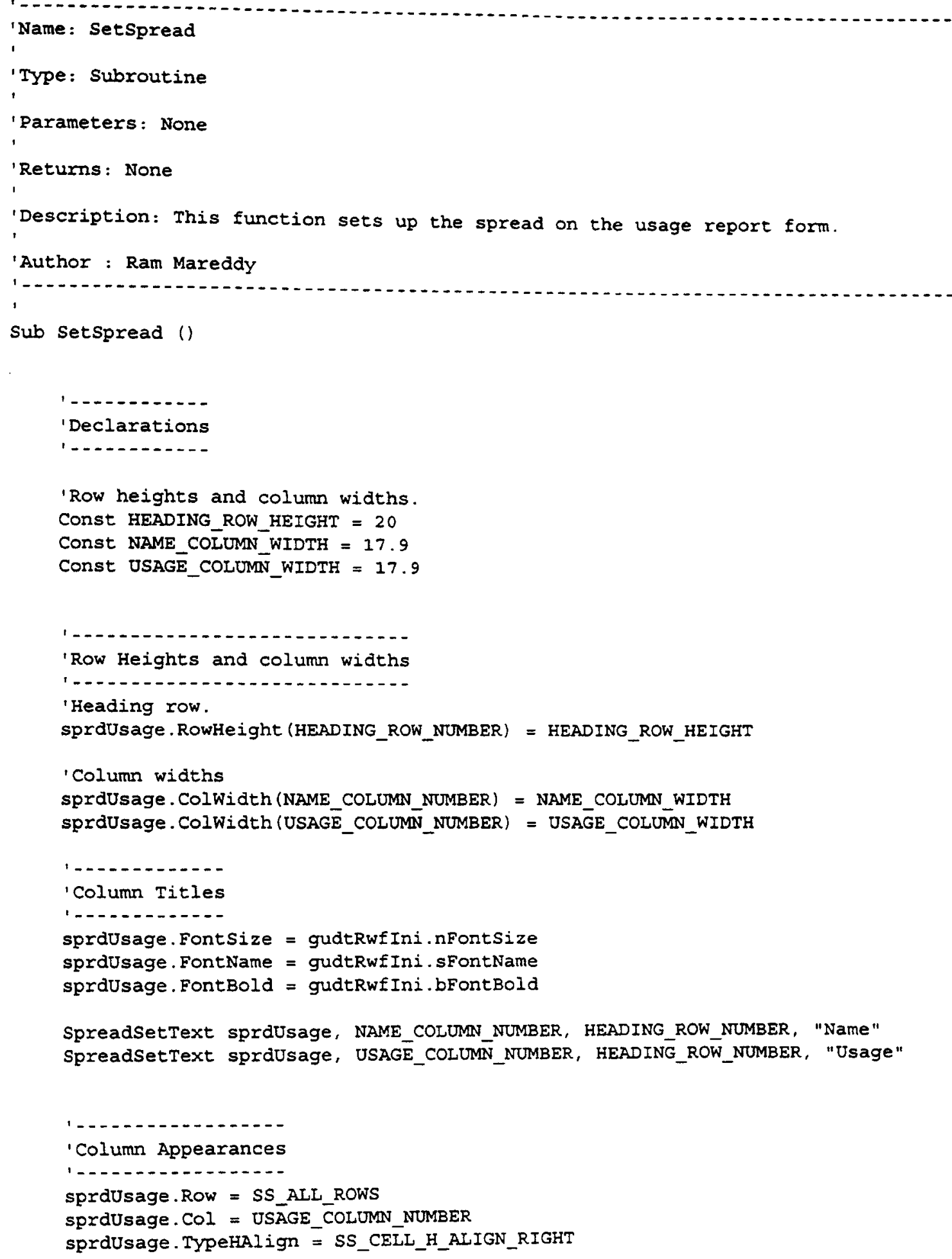

End Sub

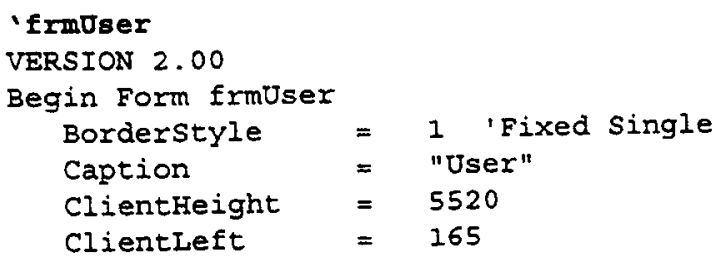




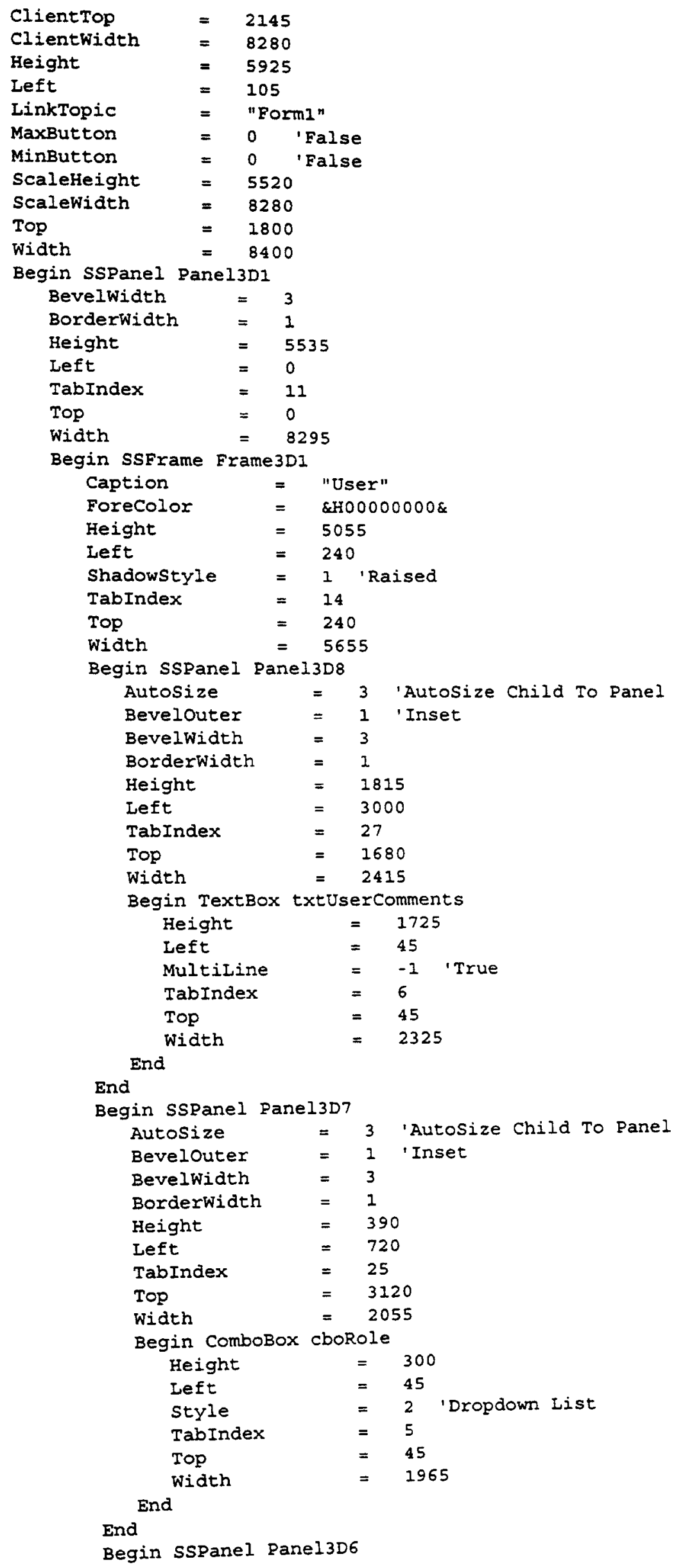




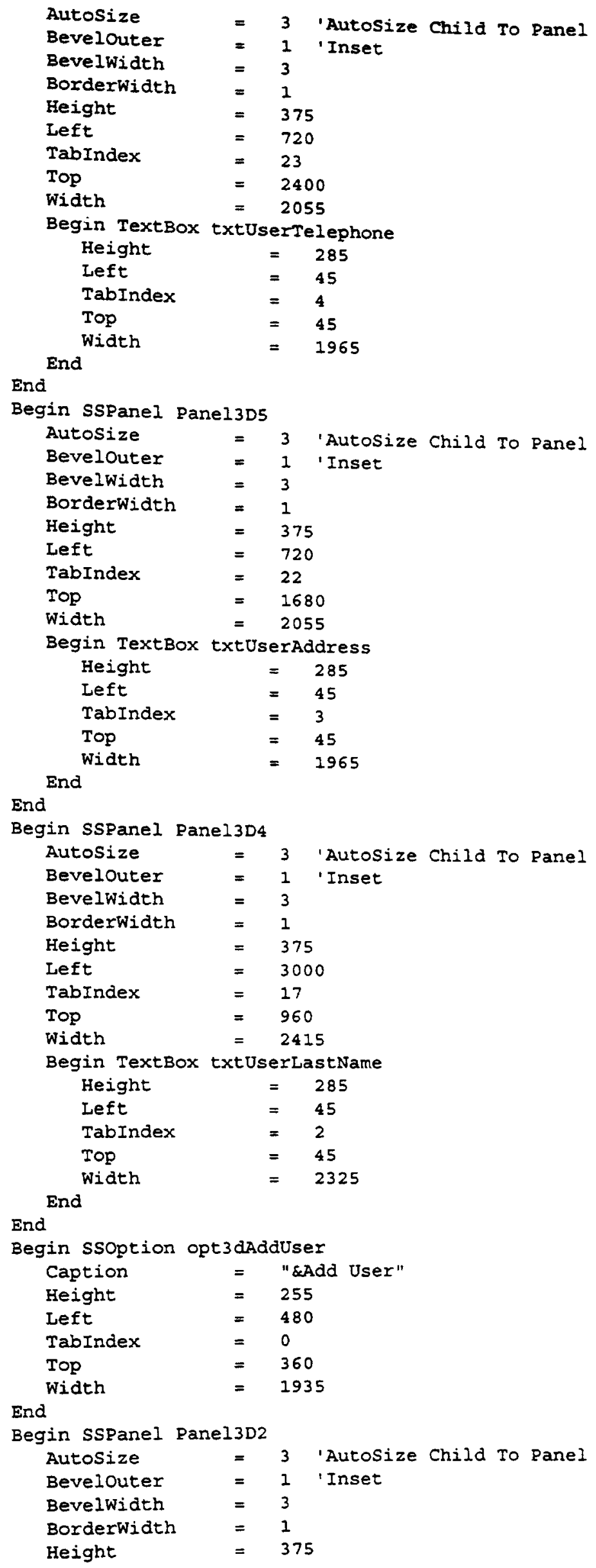




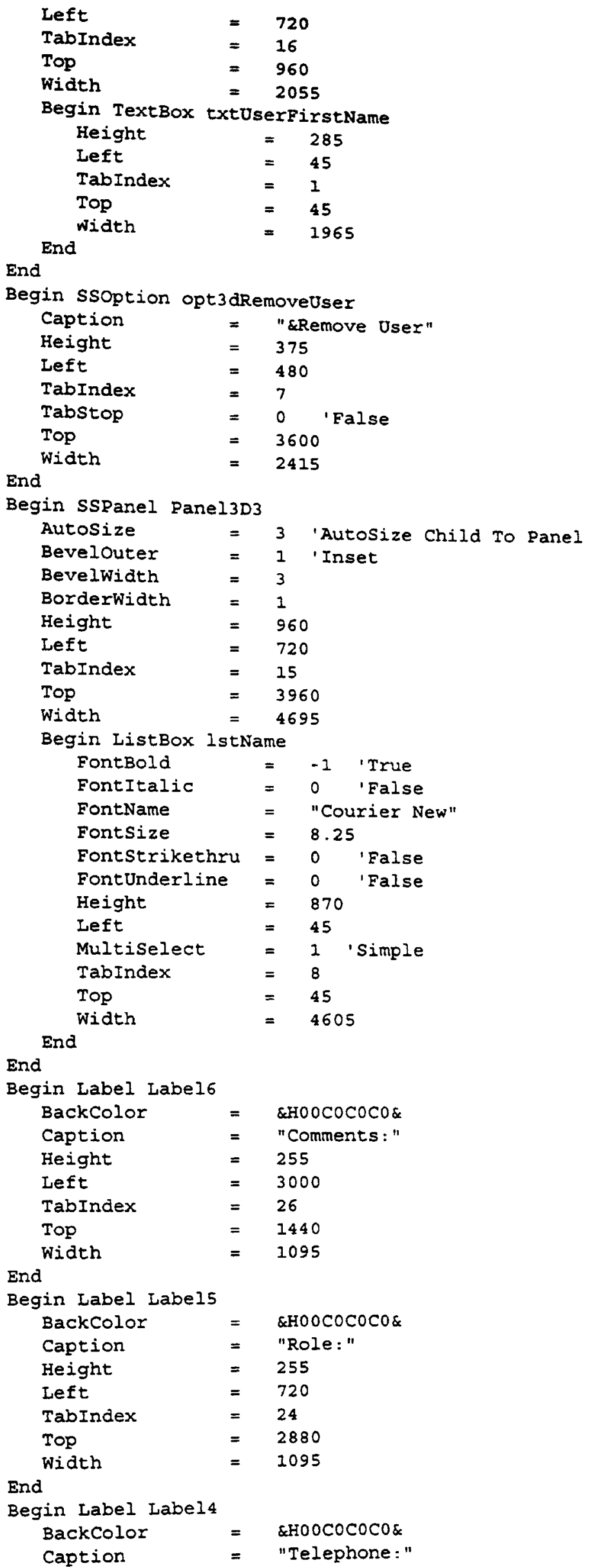




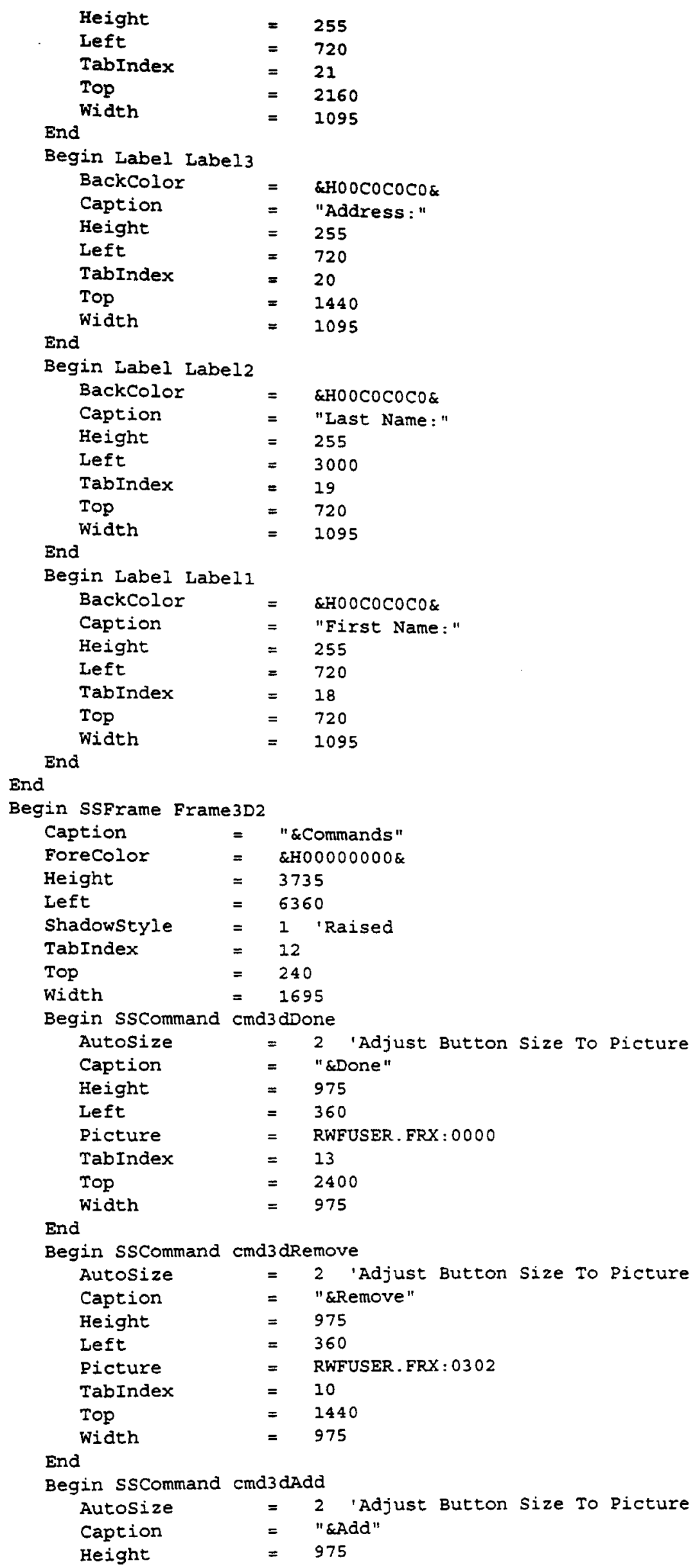




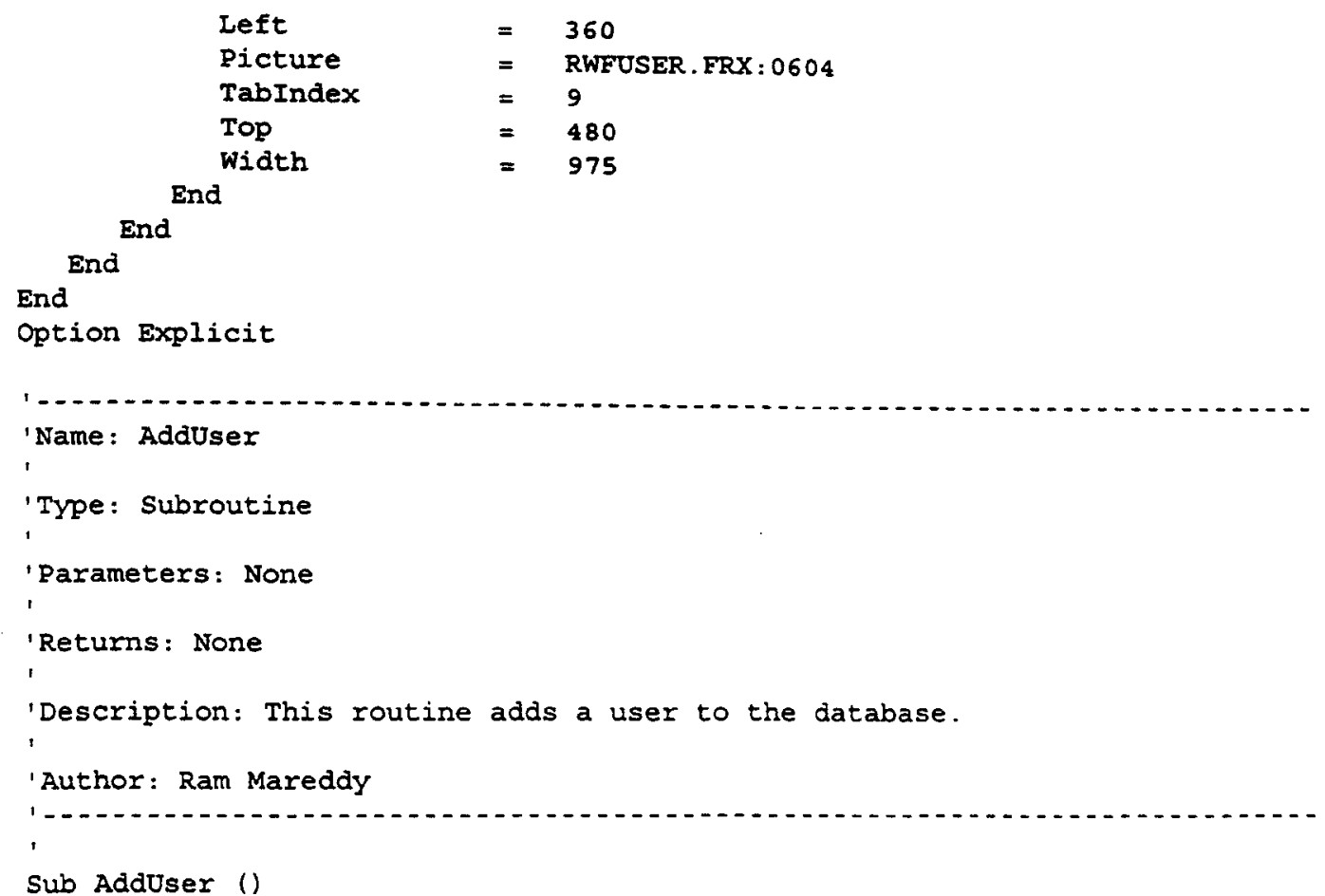

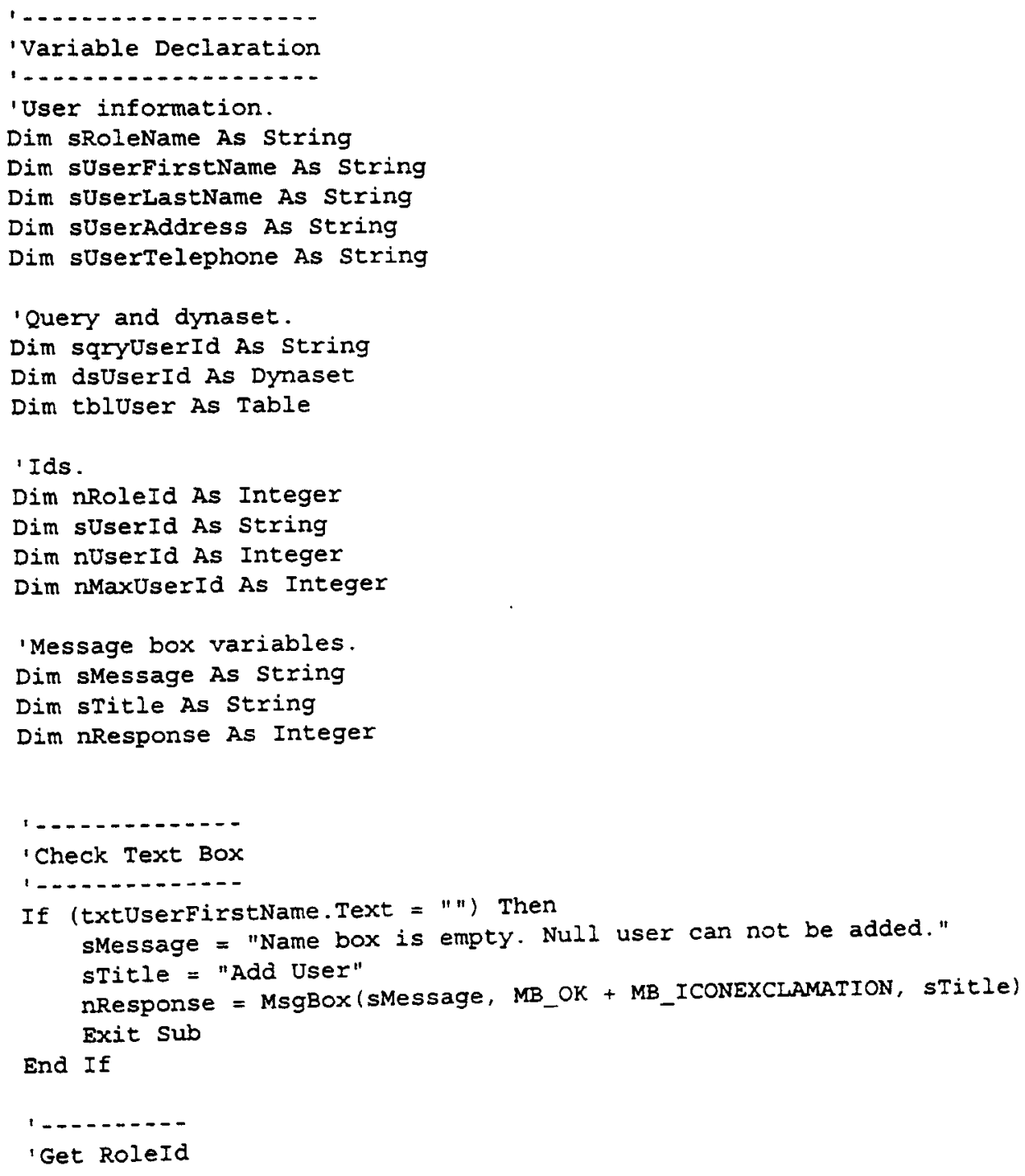

' Get RoleId 


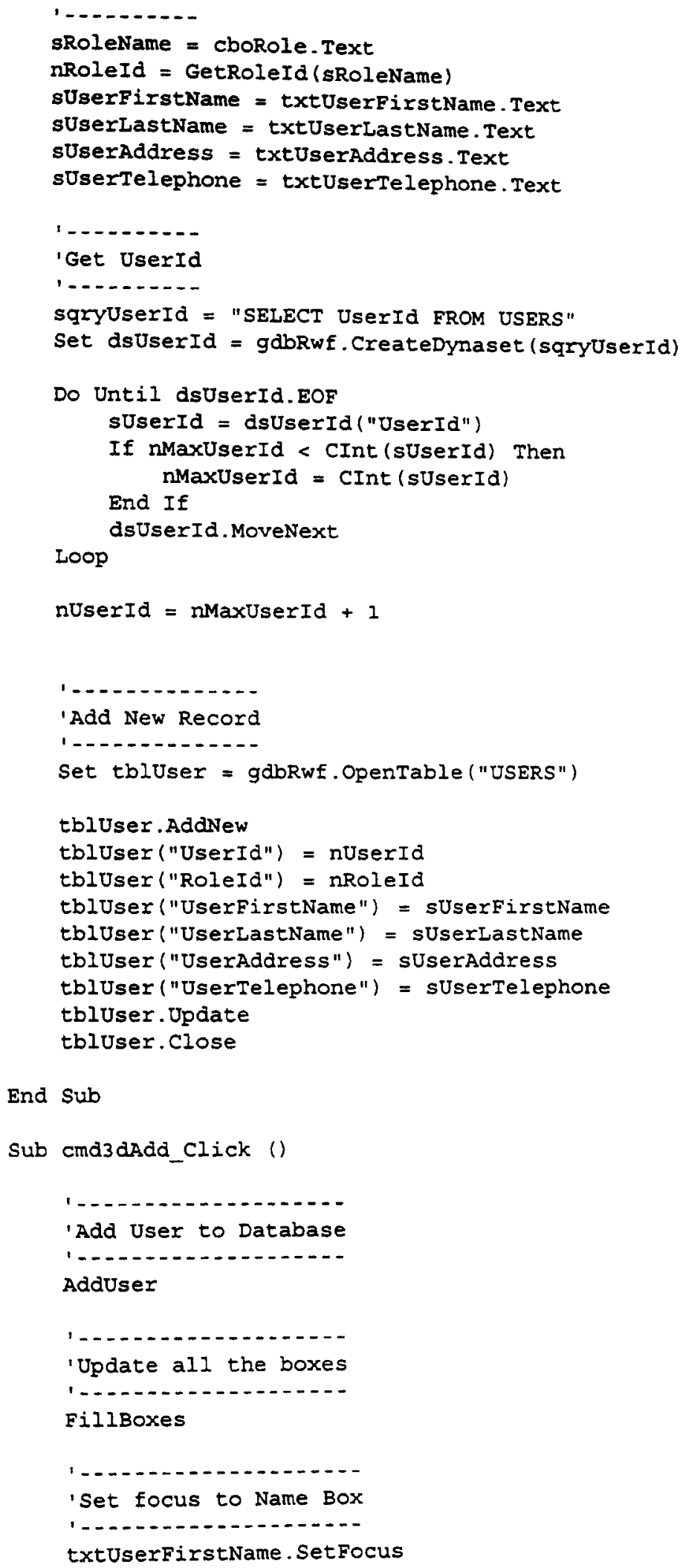


End Sub

Name: EnableRemoveuser

Type: subroutine

Parameters: None

Returns: None

'Description: This function enables all the remove user boxes and buttons,

'where as the EnableAdduser does the vice versa.

'Author: Ram Mareddy

Sub EnableRemoveUser (

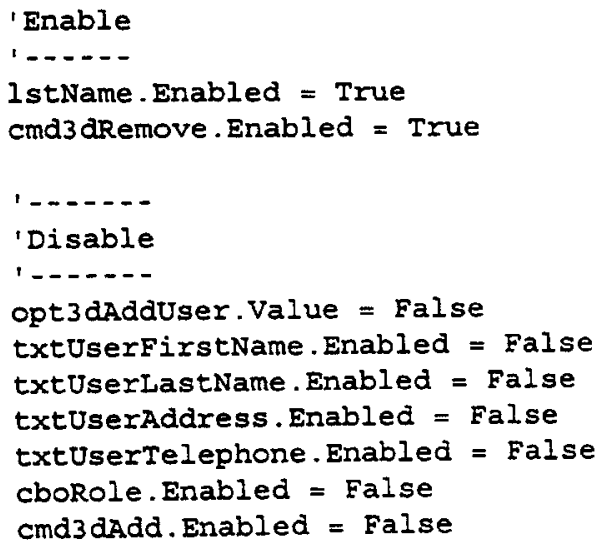

End Sub

Name: FillBoxes

Type: Subroutine

' Parameters: None

Returns: None

'Description. This subroutine fills all the boxes, i e text box, listbox with information. This can also be called after ADD or REMOVE is done.

Author: Ram Mareddy

sub FillBoxes ()

'Variable Declarations

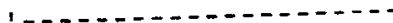

'Query strings

Dim sqryName As string

Dim sqryRole As string

Dim sName As string

'Dynasets.

Dim dsName As Dynaset

Dim dsRole As Dynaset 


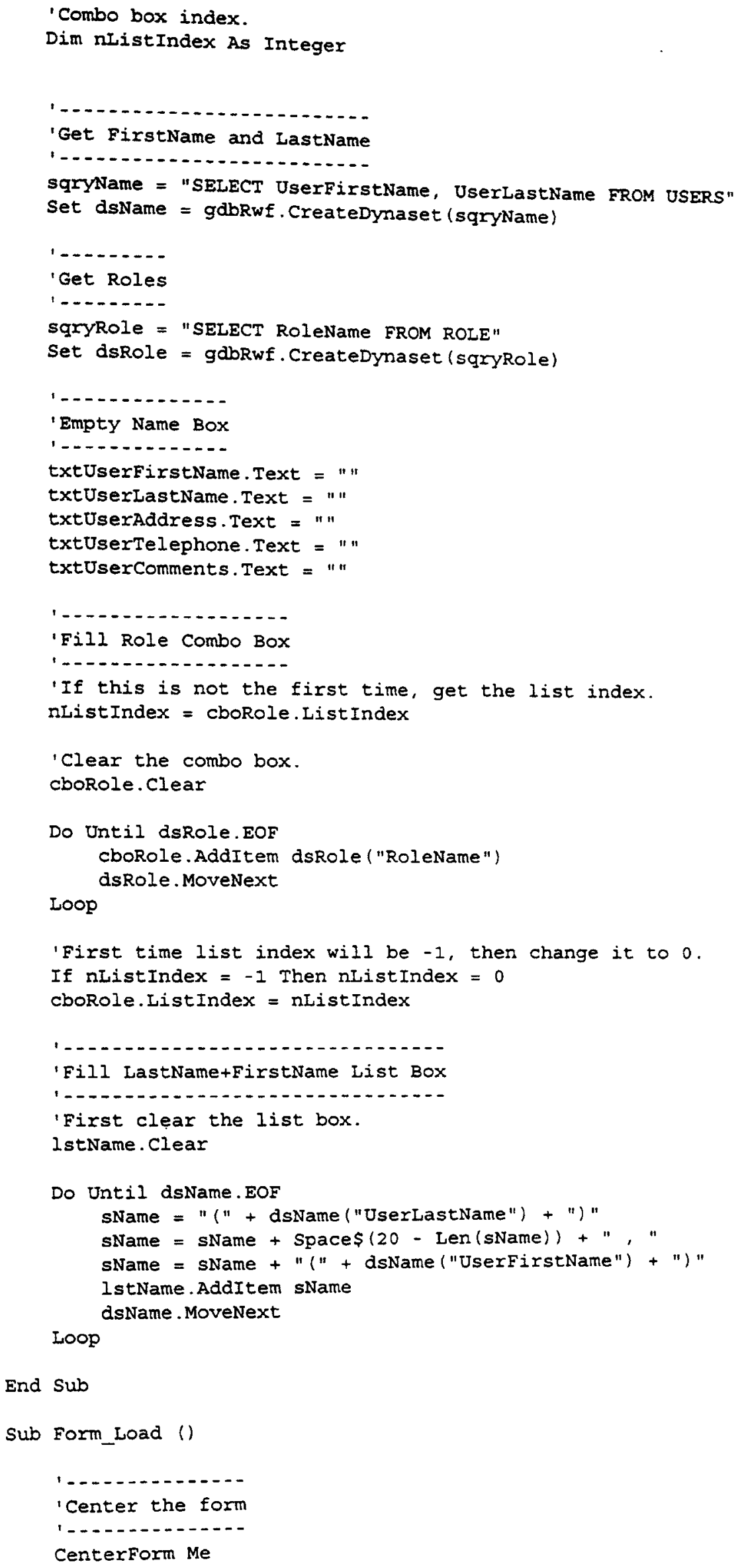


'Fill boxes on the form with details

End Sub

Sub opt3dAddUser_Click (Value As Integer)

EnableAddUser

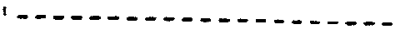

'Set focus to Name Box

1.......................

txtUserfirstName. SetFocus

End $s u b$

Sub opt3dRemoveUser_click (Value As Integer)

EnableRemoveUser

End sub

'Name: RemoveUser

'TYpe: Subroutine

'Parameters: None

'Returns: None

'Description: This function removes one or more users selected in the

remove list box.

'Author: Ram Mareddy

'

Sub Removeuser ()

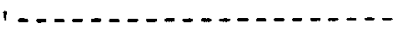

' Variable Declarations

'.....................

'Name of the user.

Dim sName As String

Dim sFirstname As String

Dim slastName As string

Dim sqryRemoveUser As String

'Positions.

Dim nstart As Integer

Dim nLength As Integer

Dim i As Integer

'All the selected users should be deleted

For $i=0$ To 1stName. Listcount - 1

If IstName.Selected $(i)=$ True Then

sName = IstName. List $(i)$

'Parse the sName into Eirst Name and Last Name.

'Format of name is (lastname), (fistname), length of name is position 2 to 


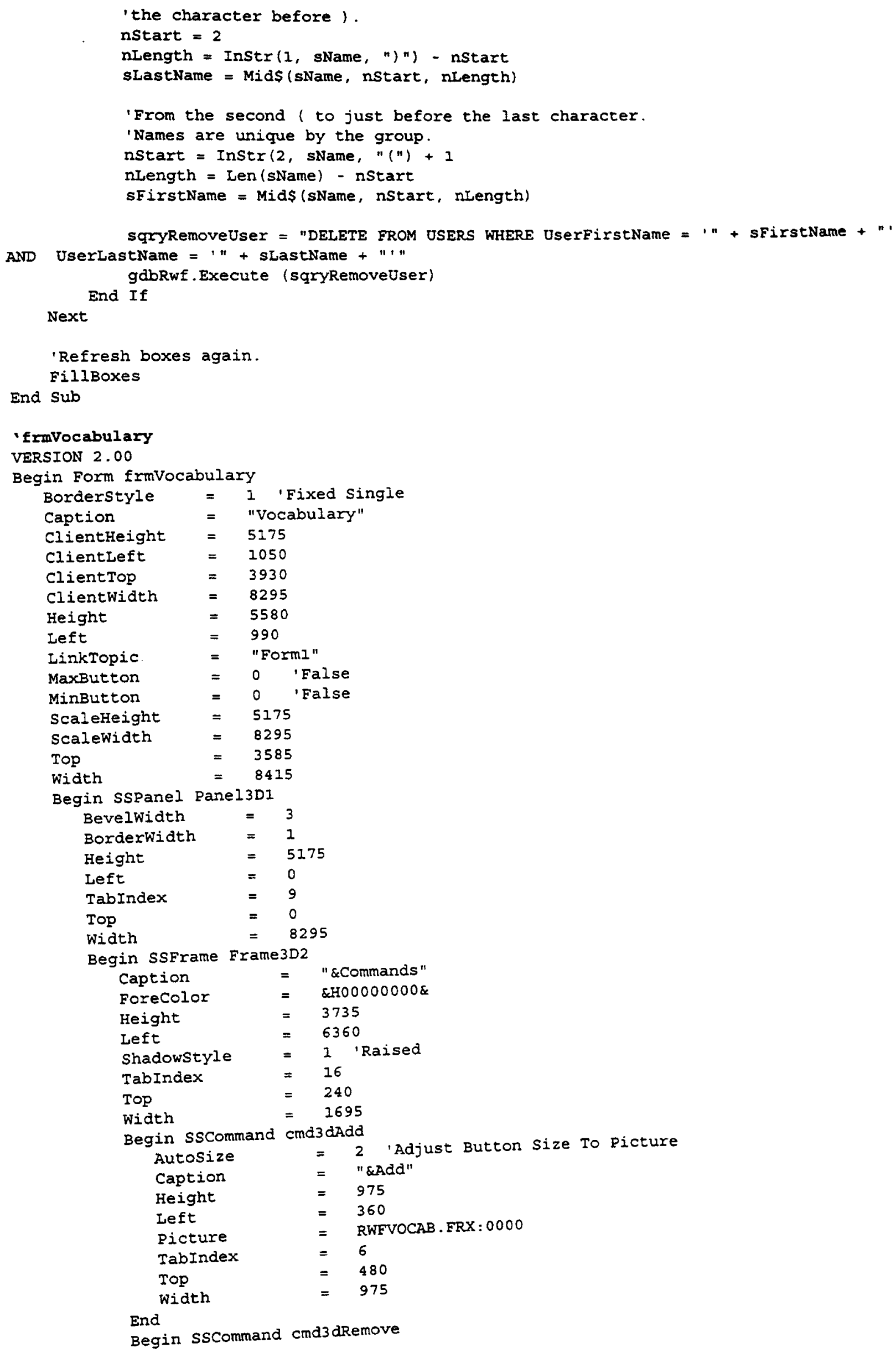




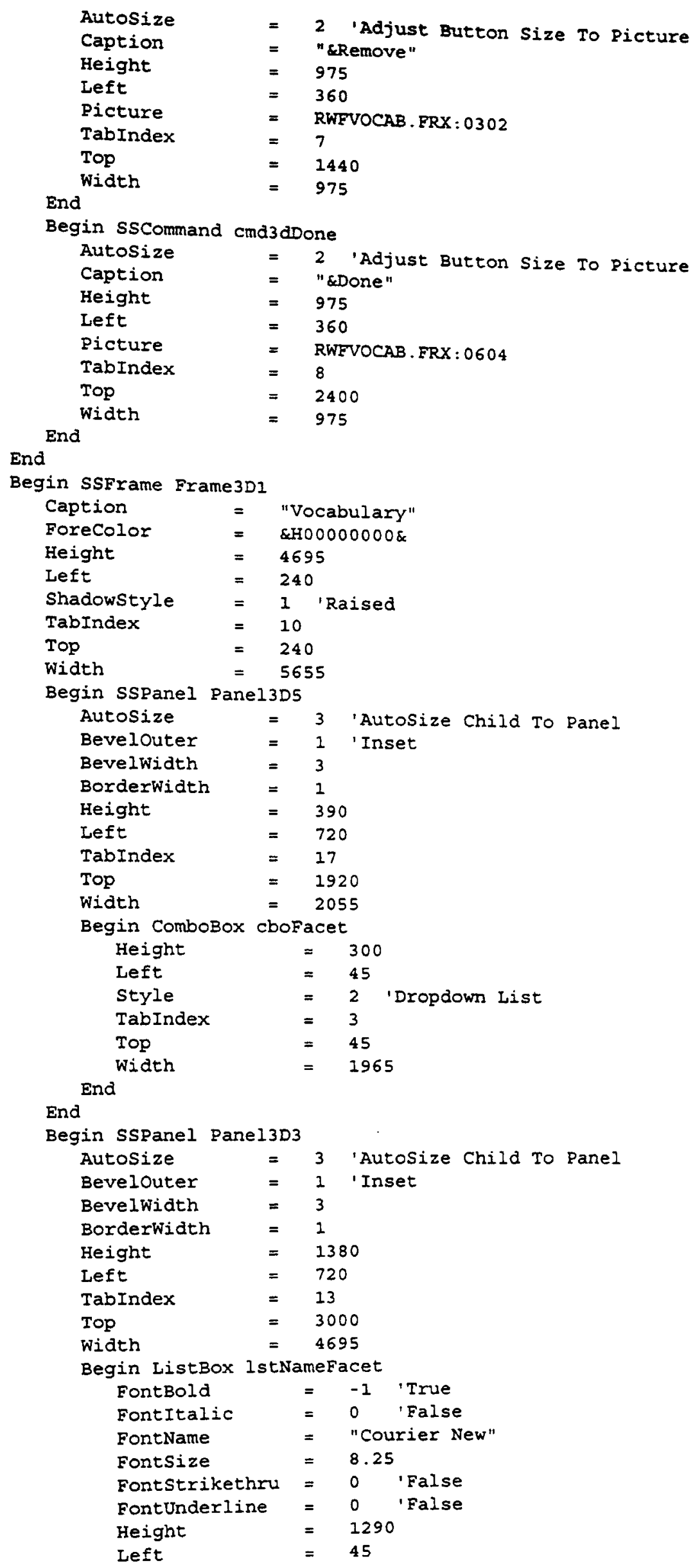




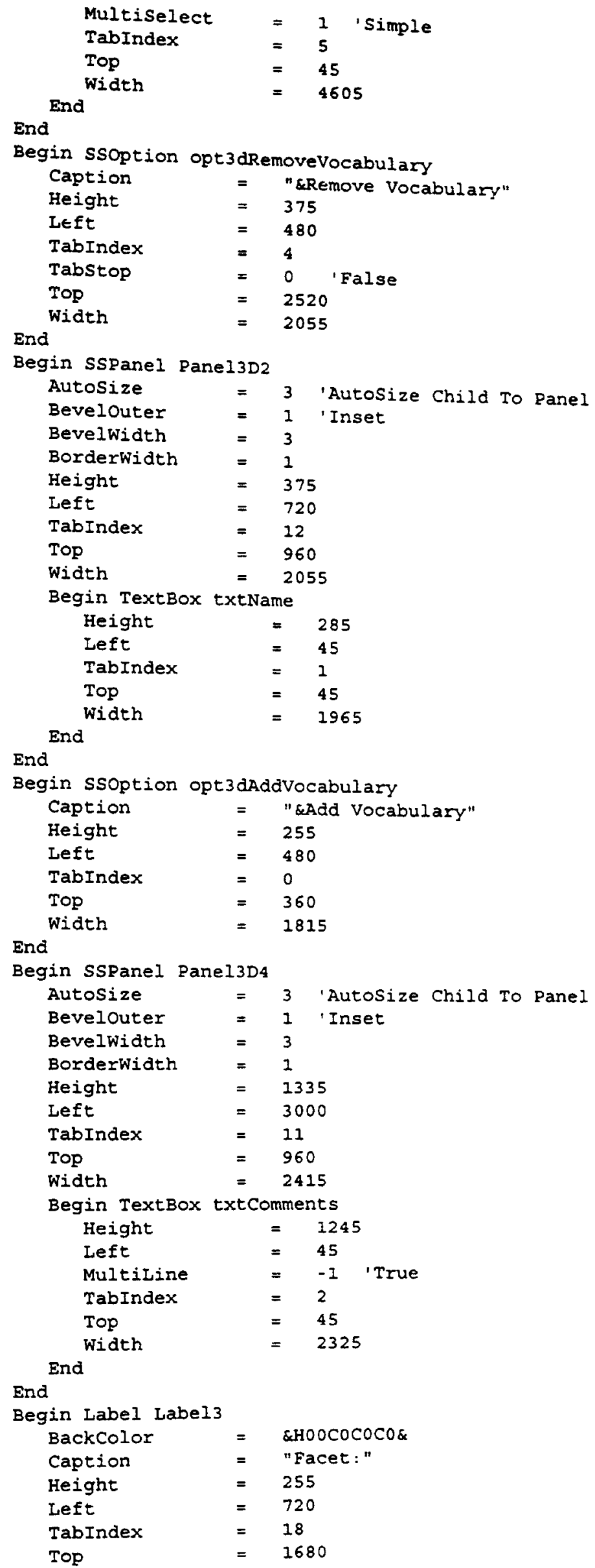




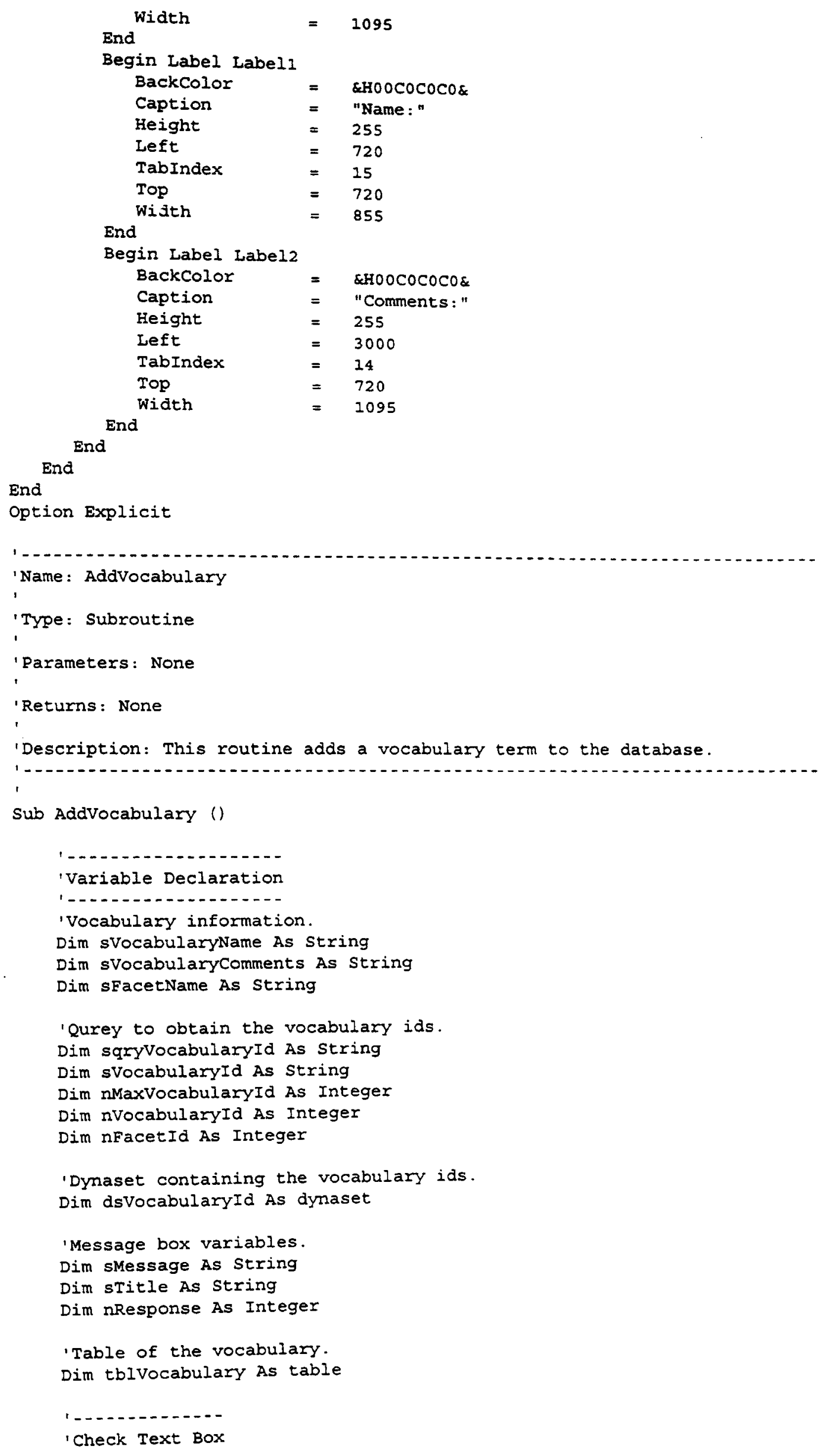




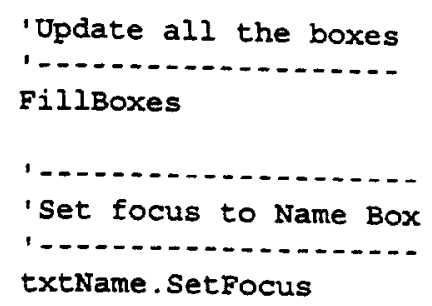

End Sub

Sub cma3dDone_Click ()

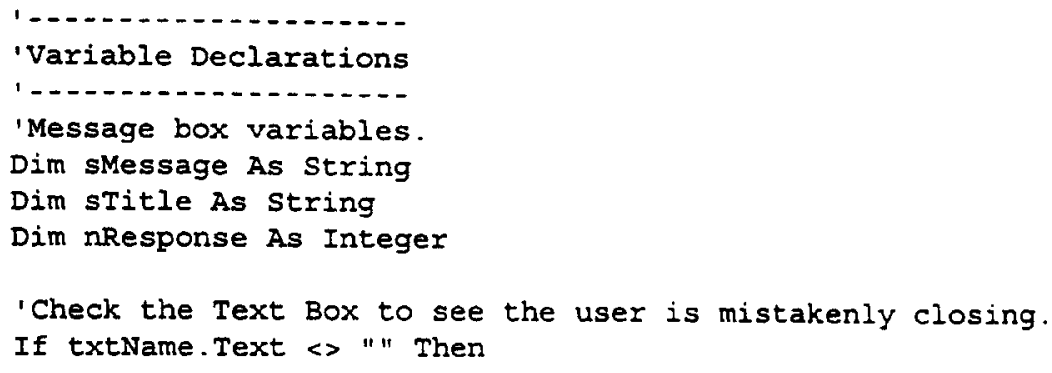


Sub EnableAddVocabulary ()

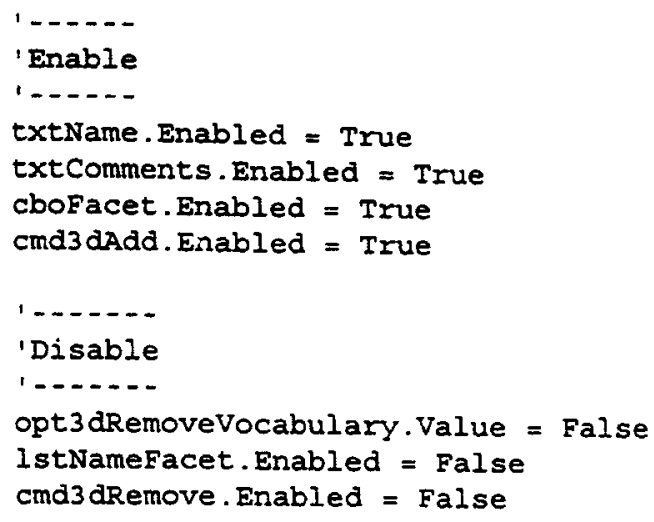

End sub

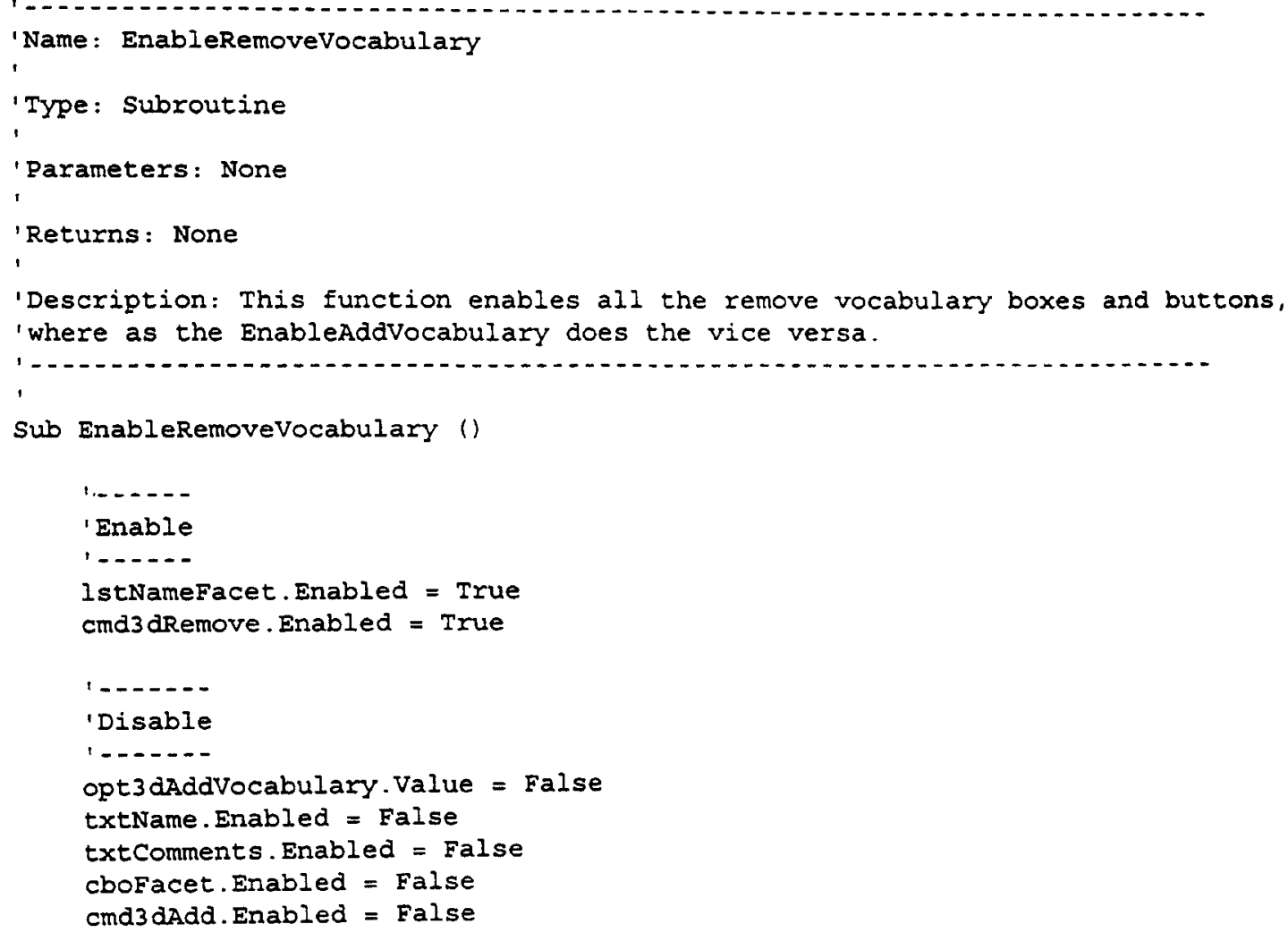

End sub

'Name: FillBoxes

'Type: Subroutine

'Parameters: None

'Returns: None

'Description: This subroutine fills all the boxes, i.e. text boxes, combobox, 'Description: This subroutine fills all the box also be called after ADD Or REMOVE is ' done. 


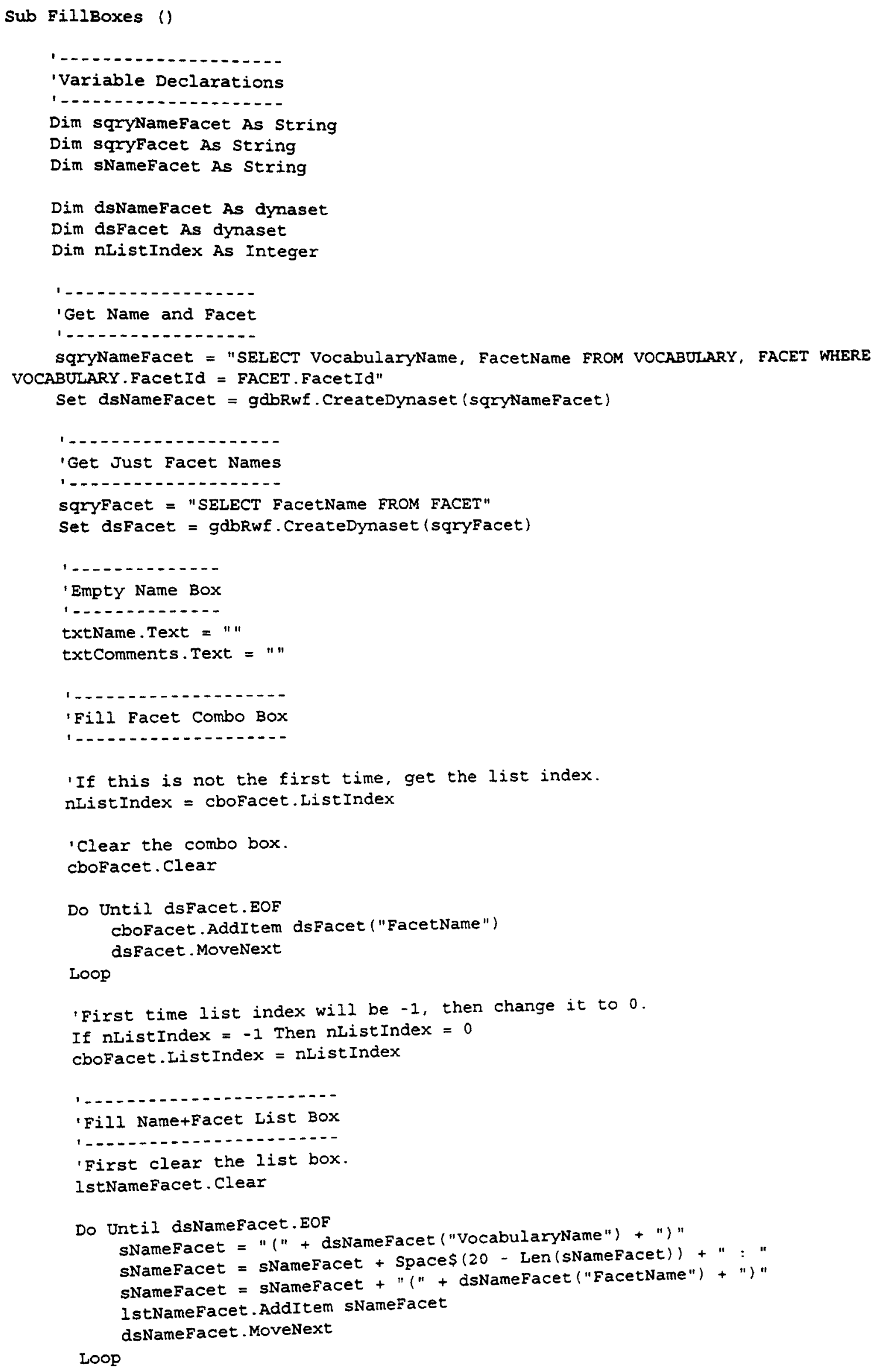

End Sub 
Sub Form_Load ()

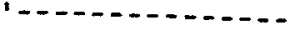

'Center the form

'

Centerform Me

1 nom

'Fill boxes on the form with details

FillBoxes

End Sub

Sub opt3dAddVocabulary_click (Value As Integer)

EnableAddVocabulary

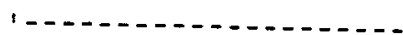

' Set focus to Name Box

'. -....................

txtName. SetFocus

End sub

Sub opt3dRemoveVocabulary_Click (Value As Integer)

EnableRemovevocabulary

End Sub

'Name: Removevocabulary

'Type: Subroutine

'Parameters: None

'Returns: None

'Description: This function removes one or more vocabulary items selected in 'the remove list box.

'Author: Ram Mareddy

Author Ram Mareddy

Sub Removevocabulary ()

1..........................

'Variable Declarations

1......................

'LOOp counter.

Dim i As Integer

' Full string in the remove box.

Dim sNamefacet As string

'Name of the vocabulary and facet.

Dim sName As String

Dim sFacet As String

Dim nFacetId As Integer

'Start and end for string parsing.

Dim nstart As Integer 
Dim nlength As Integer

'Query to remove the vocabulary.

Dim sqryRemovevocabulary As string

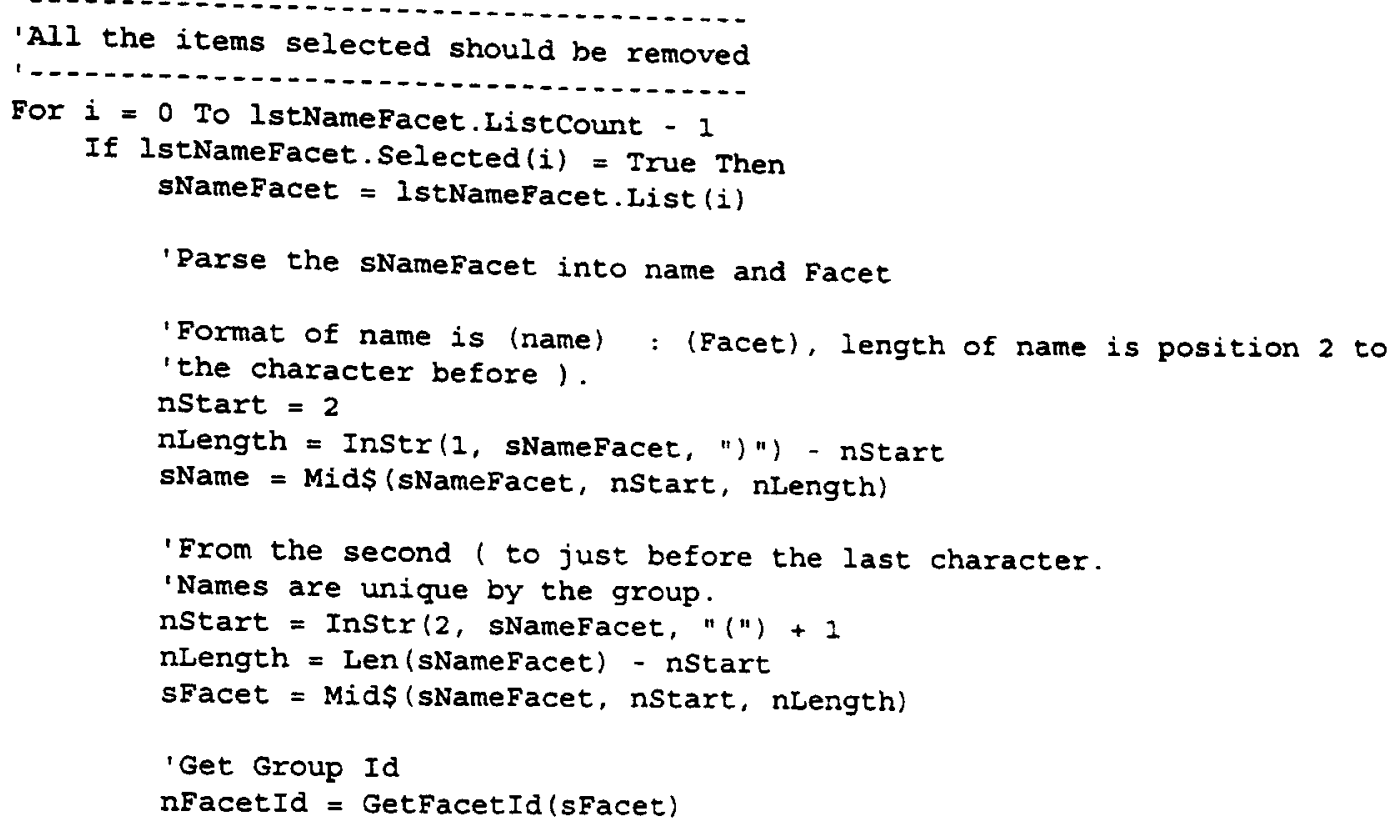

End sub

Global File

Name: Main Module

'Type: Global Declarations

'Description: All the global variables are declared here.

'Author: Ram Mareddy

' 1 -

Option Explicit 


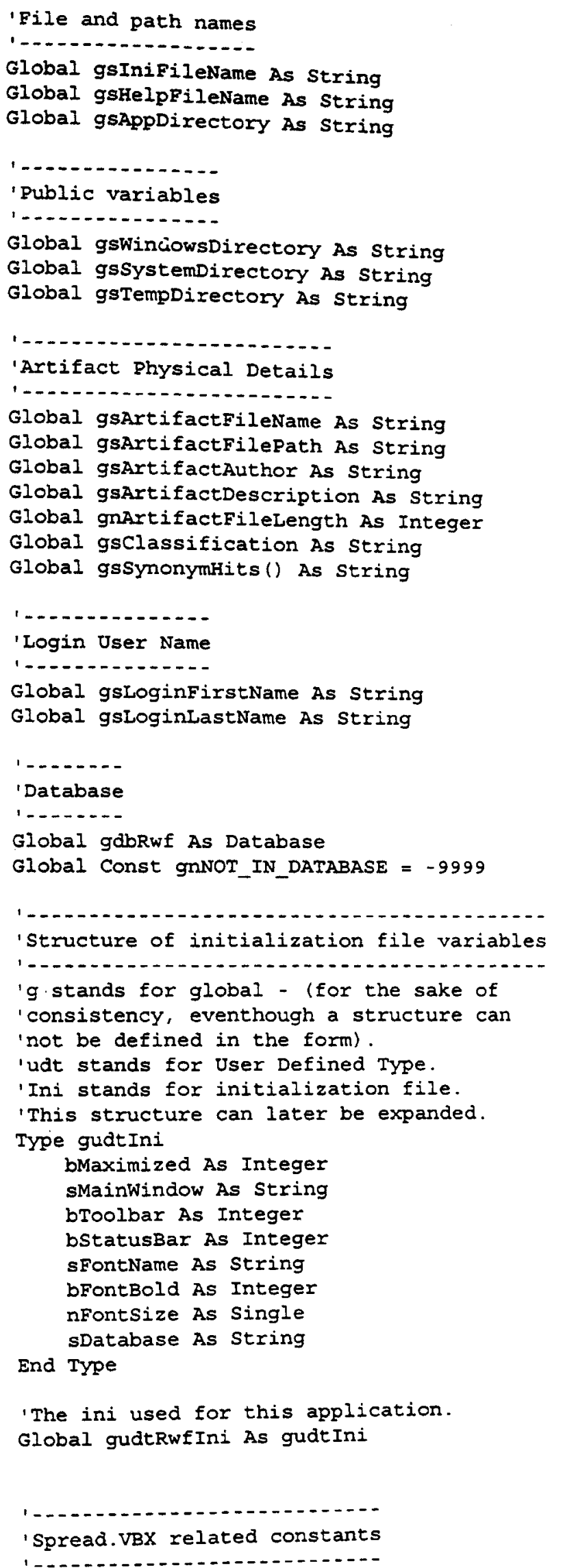

' function prototypes Declare Sub SpreadSetText Lib "Spread20.VBX" (SS As Control, ByVal Col As Long, ByVal Row As Long, lpvar As Variant) 
'All rows, all columns

Global Const SS_ALL_ROWS $=-1$

Global Const SS_ALI_COLUMavs $=-1$

'spreadsheet actions

Global Const SS_ACTION_ACTIVE_CELL $=0$

Global Const SS_ACTION_GOTO_CELL $=1$

Global Const SS_ACTION_SELECT_BLOCK $=2$

Global Const SS_ACTION_CLEAR = 3

Global Const SS_ACTION_DELETE_COI $=4$

Global Const SS_ACTION_DELETE ROW $=5$

Global Const SS_ACTION_INSERT_COL $=6$

Global Const SS_ACTION_INSERT_ROW $=7$

Global COnst SS_ACTION_LOAD_SPREAD_SHEET $=8$

Global Const SS_ACTION_SAVE_ALI $=\overline{9}$

Global Const SS_ACTION_SAVE_VALUES $=10$

Global Const SS_ACTION_RECAIC $=11$

Global Const SS_ACTION_CLEAR_TEXT $=12$

Global Const SS_ACTION PRINT $=13$

Global Const SS_ACTION_DESELECT_BLOCK $=14$

Global Const SS_ACTION_DSAVE $=\overline{15}$

Global Const SS_ACTION_SET_CELI_BORDER $=16$

Global Const SS_ACTION_ADD_MULTISELBLOCK $=17$

Global Const SS_ACTION_GET_MULTI_SELECTION $=18$

Global Const SS_ACTION_COPY_RANGE $=19$

Global Const SS_ACTION_MOVE_RANGE $=20$

Global Const SS_ACTION_SWAP_RANGE $=21$

Global Const SS_ACTION_CLIPBOARD_COPY $=22$

Global Const SS_ACTION_CLIPBOARD_CUT $=23$

Global Const SS_ACTION_CLIPBOARD_PASTE $=24$

Global Const SS ACTION SORT $=25$

Global Const SS_ACTION_COMBO_CLEAR $=26$

Global Const SS_ACTION_COMBO-REMOVE $=27$

Global Const SS_ACTION_RESET $=28$

Global Const SS_ACTION_SS_ACTION_SEI_MODE CLEAR $=29$

Global Const SS_ACTION_VMODE_REFRESH $=30^{-}$

Global Const SS_ACTION_REFRESH_BOUND $=31$

Global Const SS_ACTION_SMARTPRINT $=32$

'cell type

Global Const SS_CELL_TYPE_DATE $=0$

Global Const SS_CELL_TYPE_EDIT $=1$

Global Const SS_CELL_TYPE_FLOAT $=2$

Global Const SS_CELL_TYPE_INTEGER $=3$

Global const SS CELL TYPE PIC $=4$

Global Const SS_CELL_TYPE_STATIC_TEXT $=5$

Global Const SS_CELI_TYPE_TIME $=6$

Global Const SS_CELI_TYPE_BUTTON $=7$

Global COnst SS_CELI_TYPE_COMBOBOX $=8$

Global Const SS CELI TYPE PICTURE $=9$

Global Const SS_CELI_TYPE_CHECKBOX $=10$

Global Const SS_CELI_TYPE_OWNER_DRAWN $=11$

'sort order

Global Const SS_SORI_ORDER_NONE $=0$

Global Const SS_SORT_ORDER_ASCENDING $=1$

Global Const SS_SORT_ORDER_DESCENDING $=2$

' Sort By

Global Const SS_SORT_BY_ROW $=0$

Global Const SS_SORT_BY_COL $=1$

'date formats

Global Const SS CELI_DATE_FORMAT_DDMONYY $=0$

Global Const SS_CELL_DATE_FORMAT_DDMMYY $=1$

Global Const SS_CELL_DATE_FORMAT_MMDVY $=2$ 
Global Const SS_CELL_DATE_FORMAT_YYMMDD = 3

'Static text vertical alignment

Global Const SS_CELI_STATIC_V_ALIGN_BOTTOM =0

Global Const SS_CELI_STATIC_V_ALIGN_CENTER = 1

'Time

Global Const SS_CELL_TIME_12_HOUR_CLOCK $=0$

Global Const SS_CELI_TIME_24_HOUR_CLOCK $=1$

Unit type

Global Const SS_CELI_UNIT_NORMAL $=0$

Global Const SS_CELI_UNIT_VGA $=1$

Global Const SS_CELI_UNIT_TWIPS $=2$

'horizontal align

Global Const SS_CELI_H_ALIGN_LEFT $=0$

Global Const SS_CELI_H_ALIGN_RIGHT $=1$

Global Const SS_CELL_H_ALIGN_CENTER $=2$

'EditmodeAction

Global Const SS_CELI_EDITMODE EXIT_NONE $=0$

Global Const SS_CELI_EDITMODE_EXITUUP $=1$

Global Const SS_CELI_EDITMODE_EXIT_DOWN $=2$

Global const SS_CELL_EDITMODE_EXIT_LEFT $=3$

Global Const SS_CELL_EDITMODE_EXIT_RIGHT $=4$

Global Const SS_CELI_EDITMODE_EXIT NEXT $=5$

Global Const SS_CELI_EDITMODE_EXIT_PREVIOUS $=6$

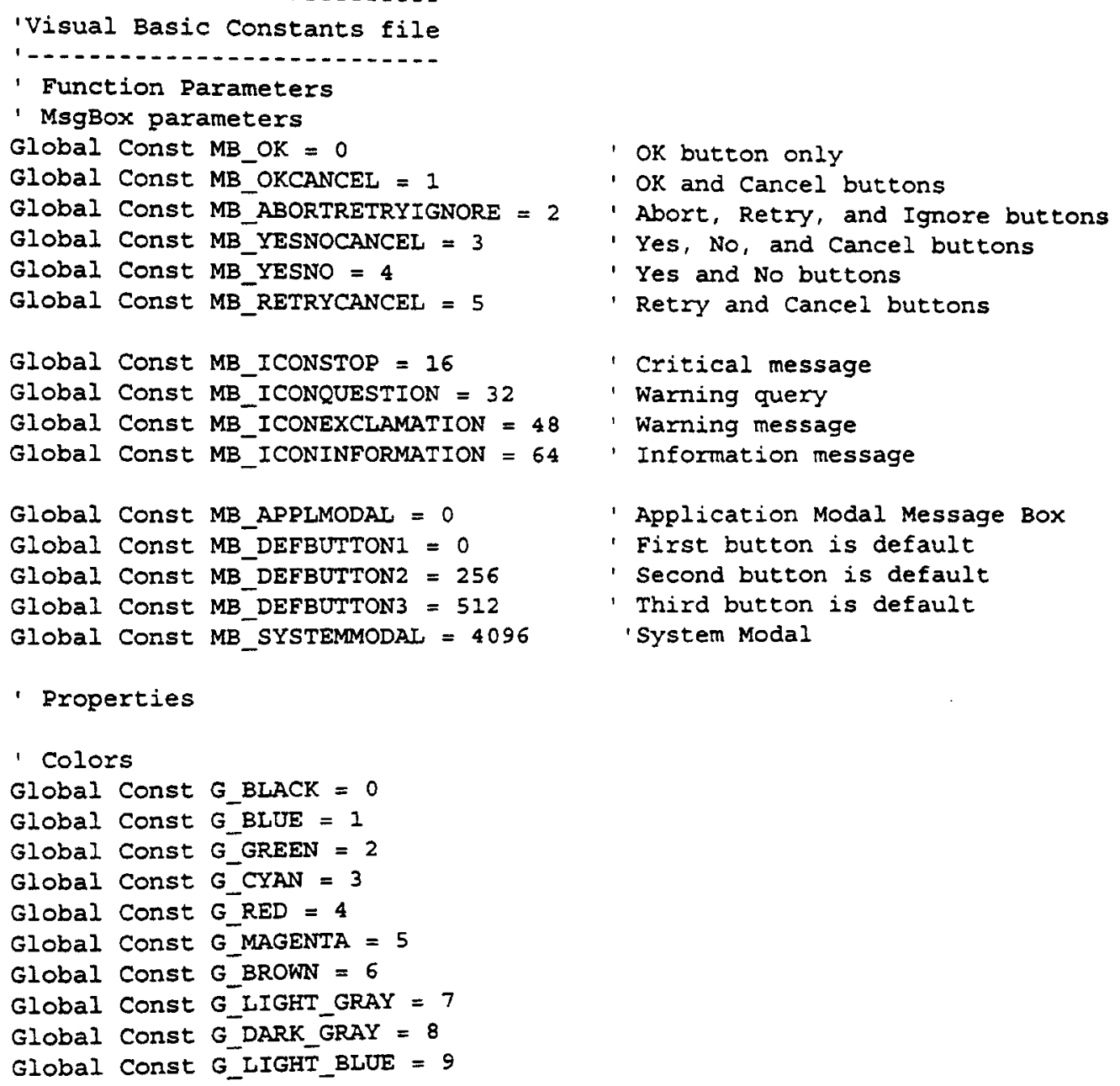




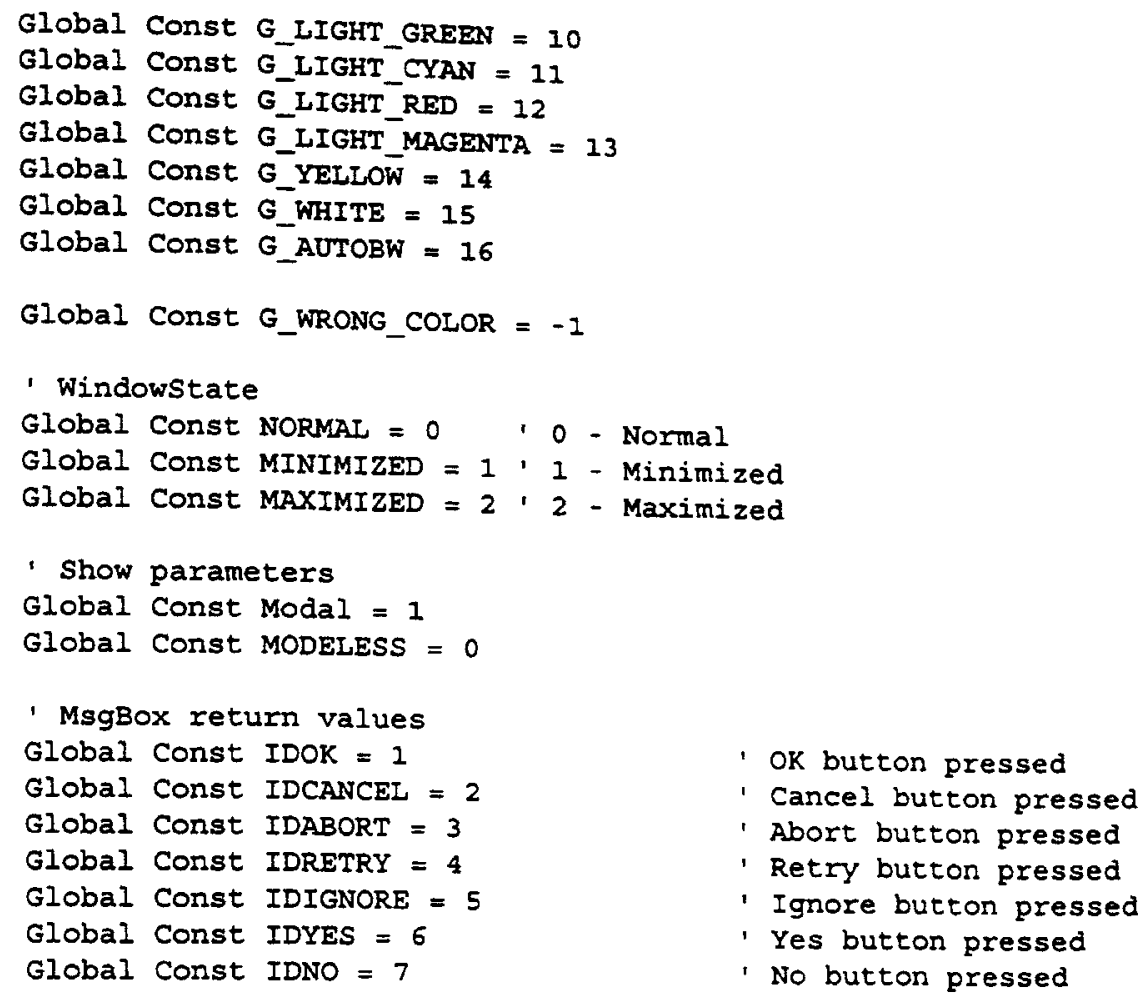

'Windows API function declarations

'Windows apI function declarations

Declare Function GetPrivateProfilestring Lib "Kernel" (ByVal lpApplicationName As String, lpKeyName As Any, ByVal lpDefault As String, ByVal lpReturnedstring As String, ByVal nsize As Integer, Byval lpFilename As String) As Integer

Declare Function GetPrivateProfileInt Lib "Kernel" (Byval lpApplicationName As String. ByVal lpKeyName As String, ByVal nDefault As Integer, ByVal lpFileName As String) As Integer

Declare Function GetwindowsDirectory Lib "Kernel" (ByVal lpBuffer As String, Byval nsize As Integer) As Integer

Declare Function GetSystemDirectory Lib "Kernel" (ByVal lpBuffer As String, ByVal nSize As Integer) As Integer

Declare Function WritePrivateprofileString Lib "Kernel" (ByVal lpApplicationName As

String, lpKeyName As Any, lpstring As Any, ByVal lplfileName As String) As Integer

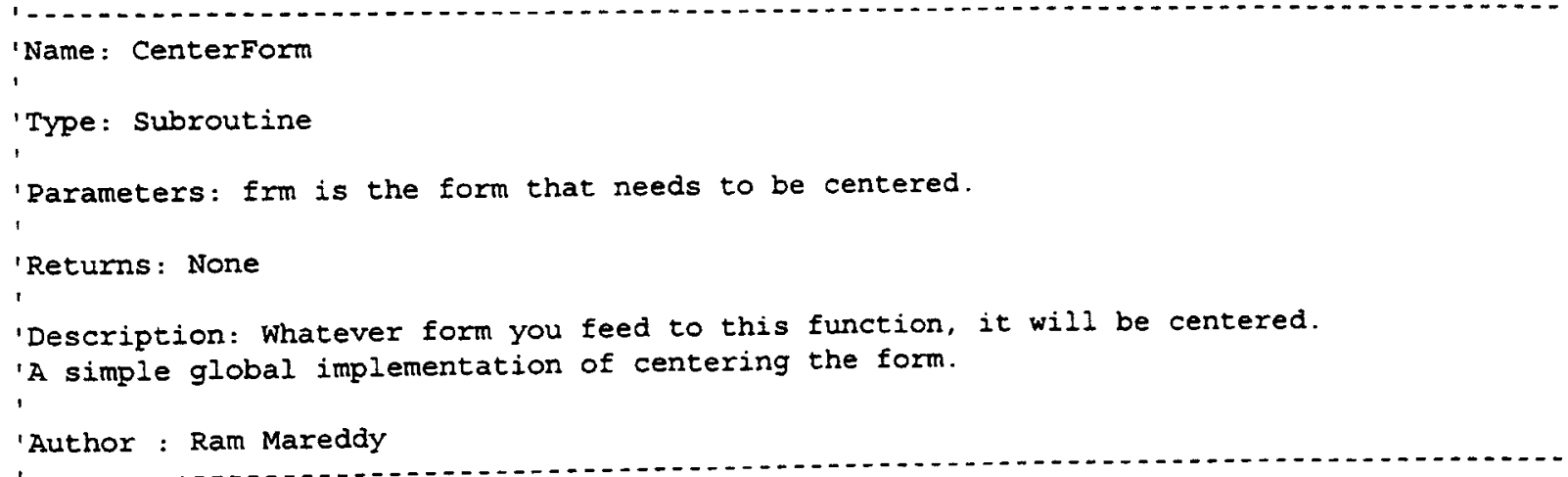


End Sub

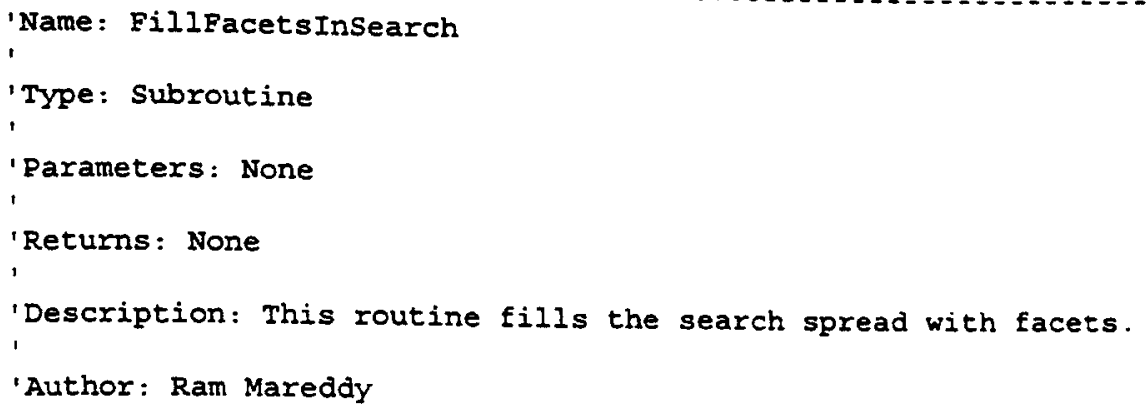




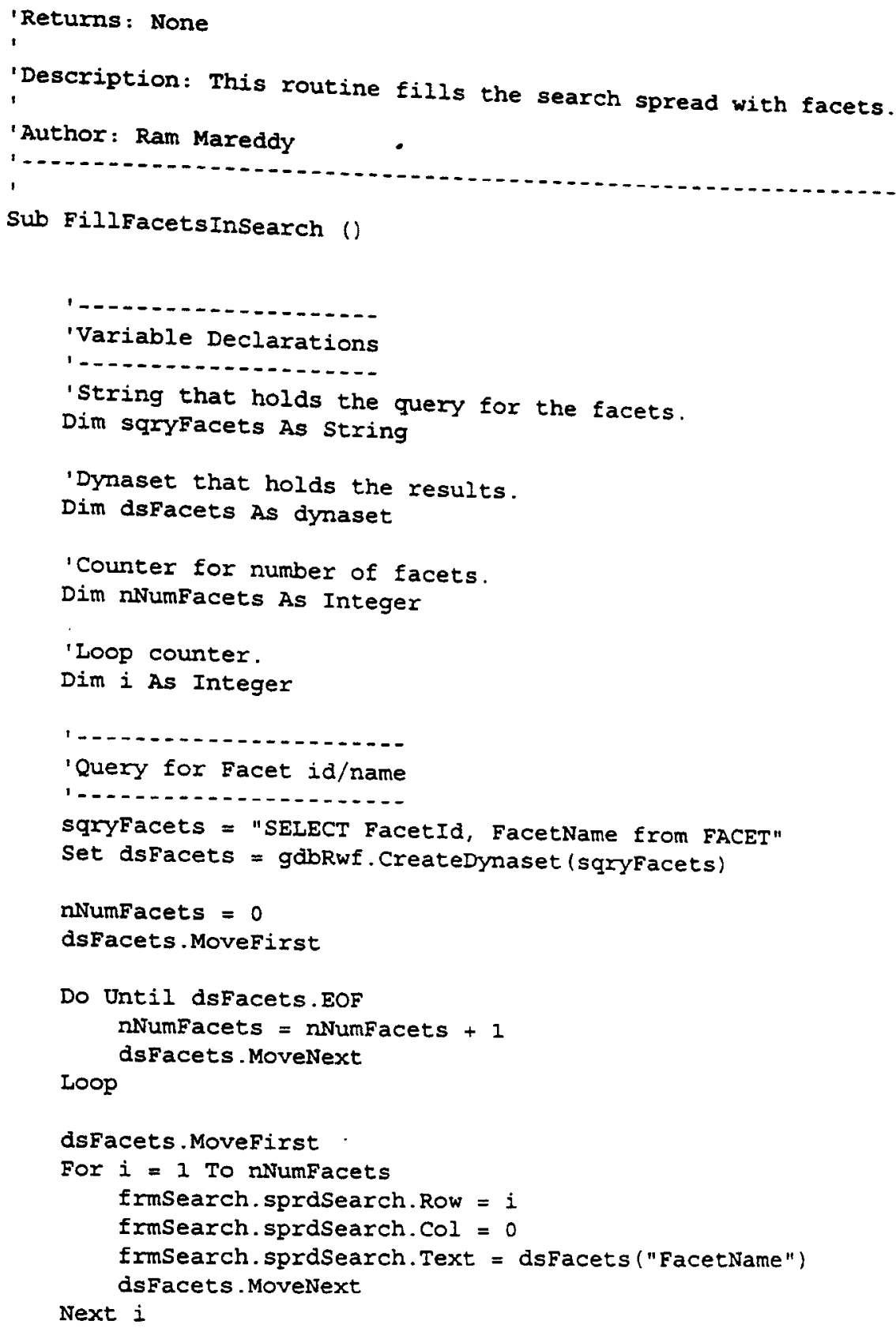




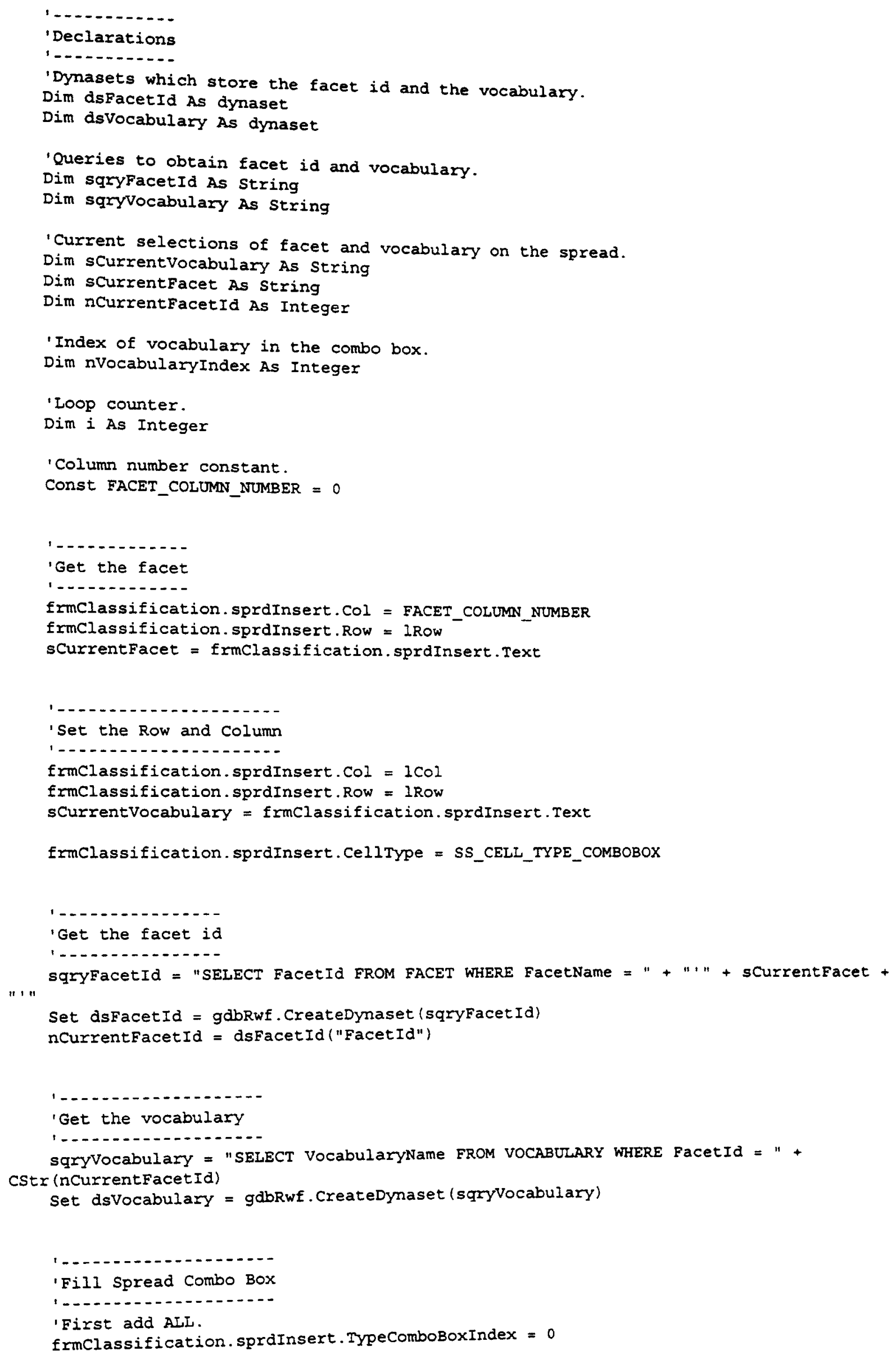




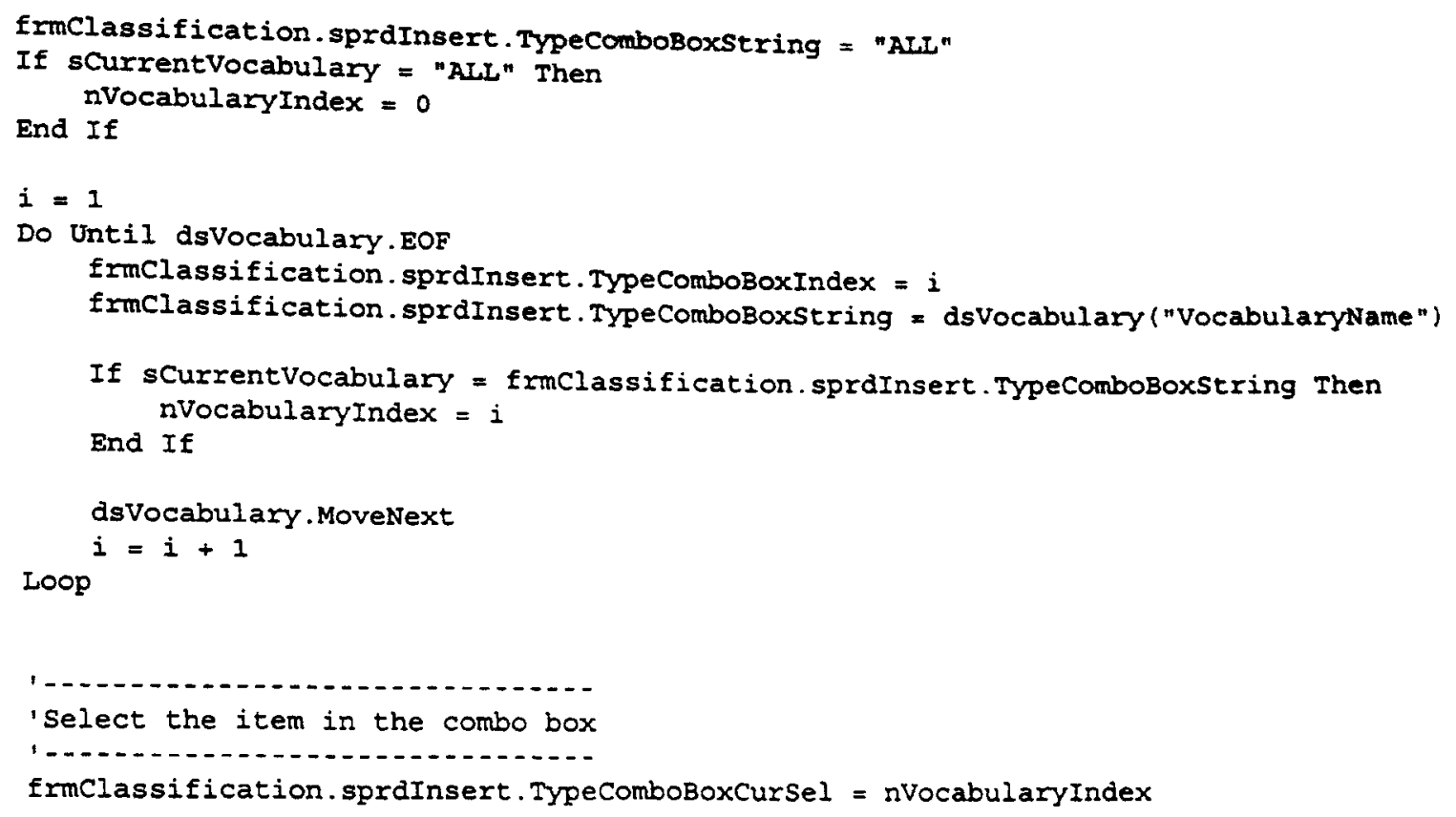

End Sub

'Name: FillspreadCombo

'Type: Subroutine

'Parameters: ICol is the column number of the cell and lRow is Row number.

'Returns: None

'Description: This function fills the combobox on the spread, for that 'particular selected cell.

' Author: Ram Mareddy

Sub FillsearchCombo ( $1 \mathrm{Col}$ As Long, 1Row As Long)

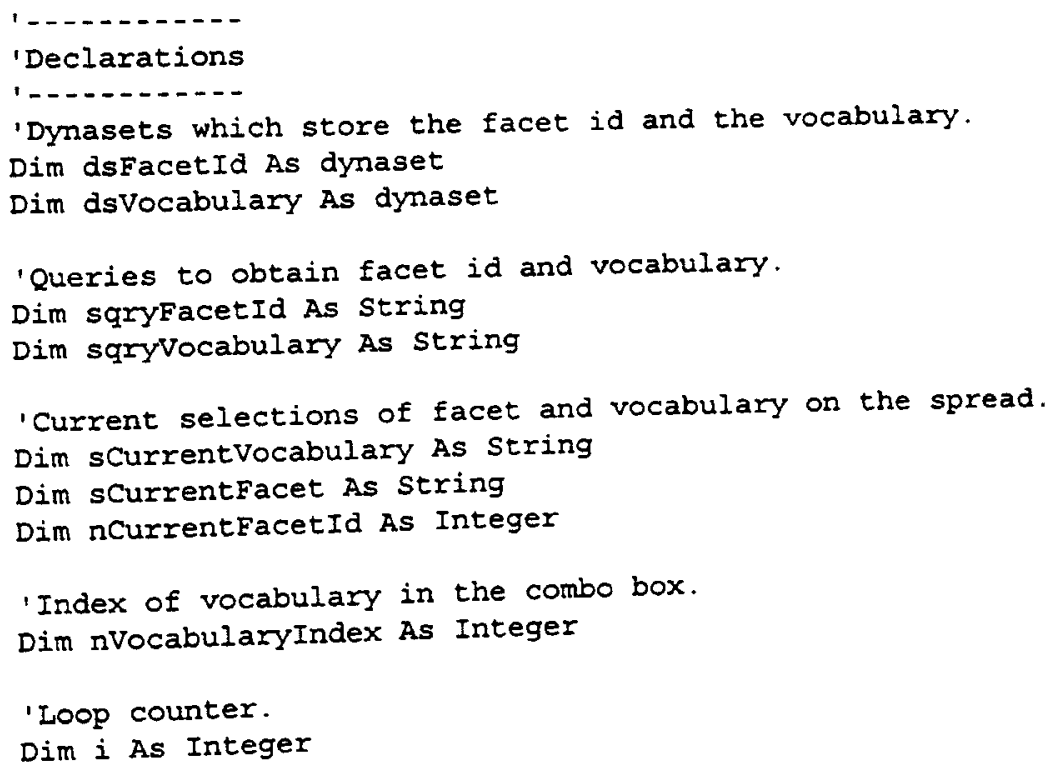


' Column number constant.

Const FACET_COLUMN_NOMBER $=0$

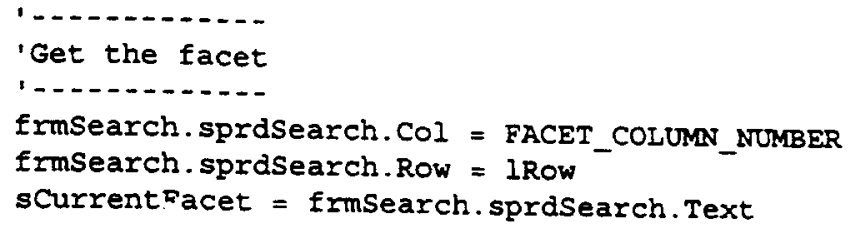




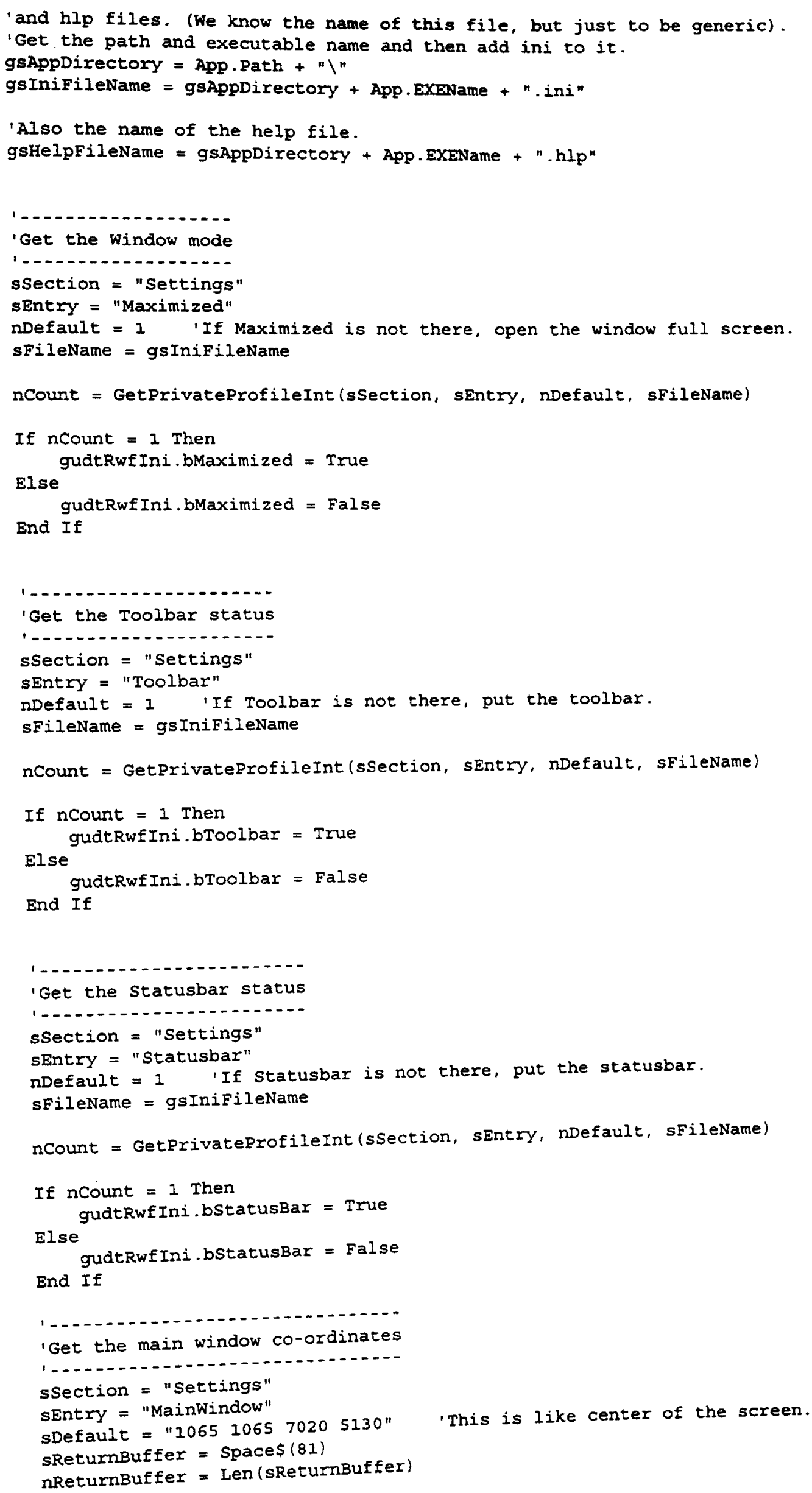


sFileName = gsInifileName

nCount = GetPrivateProfilestring(sSection, Byval sEntry, sDefault, sReturnBuffer, nReturnBuffer, sFileName)

gudtRwfIni. sMainwindow = Left $\$$ (sReturnBuffer, ncount)

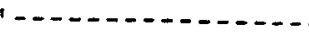

'Get the font name

1.....................

ssection = "Settings"

sEntry = "FontName"

sDefault = "MS Sanserif"

sReturnBuffer = Space $\$(81)$

nReturnBuffer = Len (sReturnBuffer)

sFileName = gsIniFileName

nCount = GetPrivateprofilestring(sSection, ByVal sentry, sDefault, sReturnBuffer, nReturnBuffer, sFileName)

gudtRwfIni.sFontName = Left\$(sReturnBuffer, nCount)

1..-.................

sSection = "Settings"

sEntry = "FontBold"

nDefault $=0$ 'If bold status is omitted, do not use bold.

sFileName $=$ gsIniFileName

ncount = GetPrivateProfileInt(sSection, sEntry, nDefault, sfileName)

If ncount $=1$ Then

gudtRwfIni.bFontBold = True

Else

gudtRwfIni bFontBold = False

End If

'Get font size

1...........-.

ssection = "Settings"

sEntry = "FontSize"

ndefault $=8$ 'If font size is omitted, use size 8

sFilename = gsIniFileName

ncount = GetPrivateProfileInt (sSection, sEntry, nDefault, sFileName)

gudtRwfIni.nFontSize $=$ ncount

'Get the default datasource

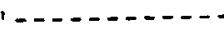

ssection = "Datasource"

sEntry = "Default"

sDefault $=" "$

'When there is no database specified, we are in

trouble. We have to warn the user and quit.

sReturnBuffer $=$ Space $\$(81)$

nReturnBuffer = Len (sReturnBuffer)

sFileName = gsIniFileName

nCount = GetPrivateProfilestring (ssection, ByVal sEntry, sDefault, sReturnBuffer, nReturnBuffer, sFilename) 
gudtRwfIni.sDatabase $=$ Left $\$$ (sReturnBuffer, nCount)

'If database is not specified...

sMessage = "The default database file is not specified."

sMessage = sMessage + "Under [Datasource] section in the initialization file add

Default $=<$ Database Name $>$.

sMessage = sMessage + "Initialization file name is " + gsInifileName + "."

sTitle = "Initialization Exror"

If gudtRwfIni.sDatabase $=$ " "Then

nResponse $=$ MsgBox (sMessage, MB_OK + MB_ICONSTOP, sTitle)

End

End If

End Sub

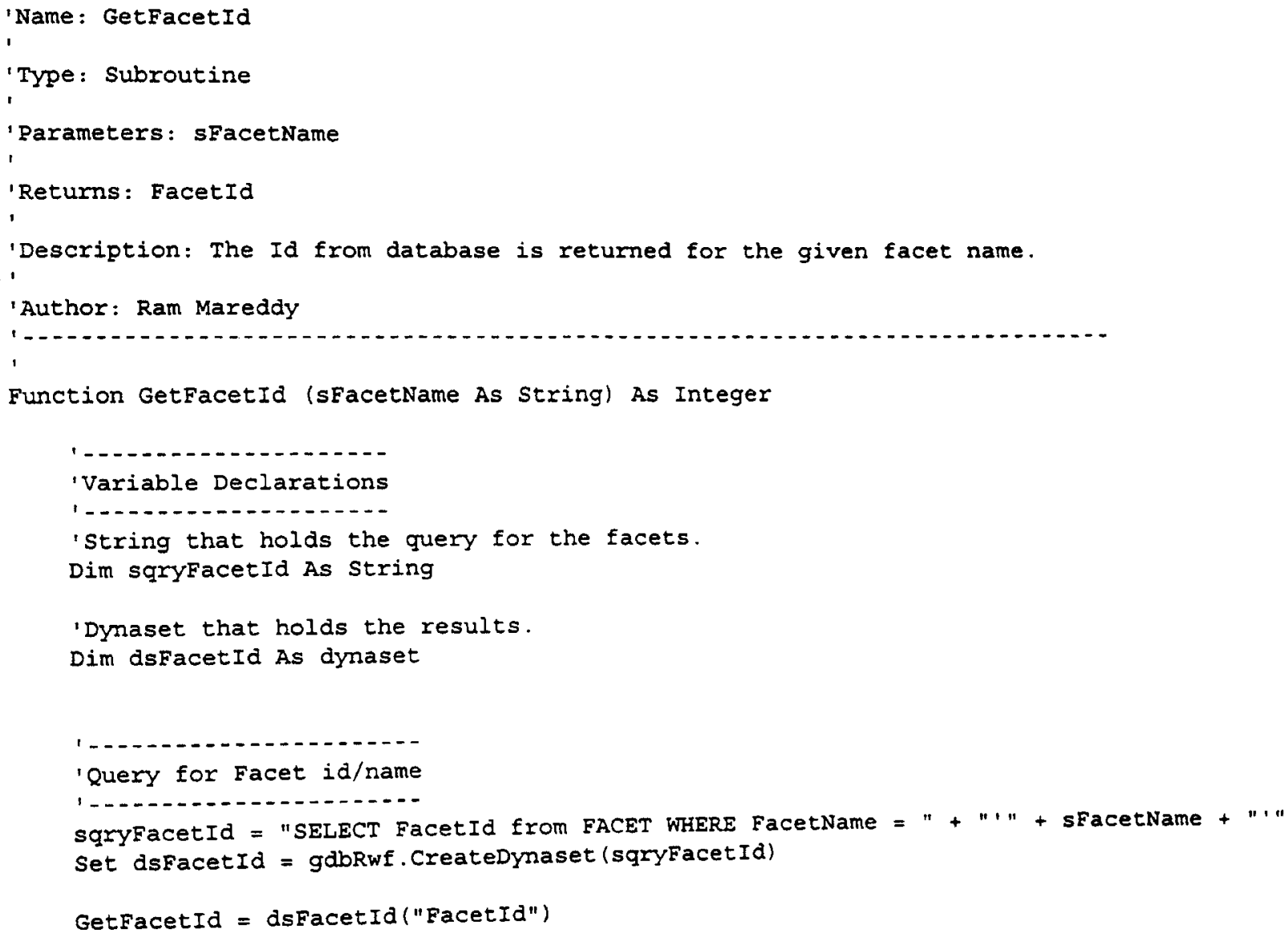




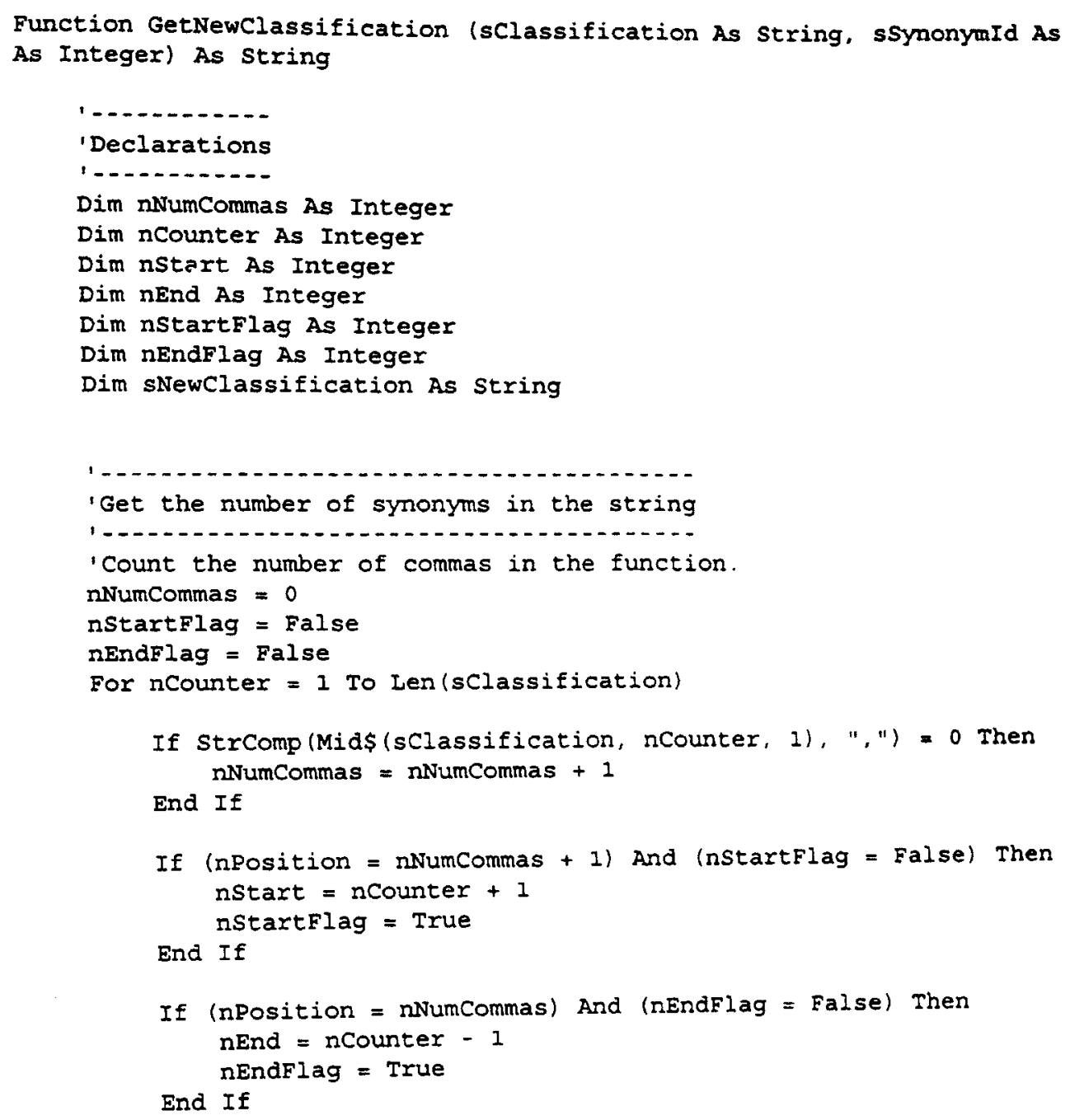

Next

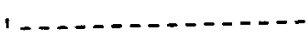

'Adust the string

sNewclassification = Left\$(sClassification, nstart - 1 )

sNewClassification = sNewClassification + sSynonym

( sNew

GetNewClassification = sNewClassification

End Function

'Name: GetNthstring

'Type: Function

Parameters. sstring is the string to be separated, nN is the number of string

' Parameters: sstring is the string to separator string.

'Retunxs: Nth string segment of the parent string.

'Description: This function returns part of the string which is divided by 


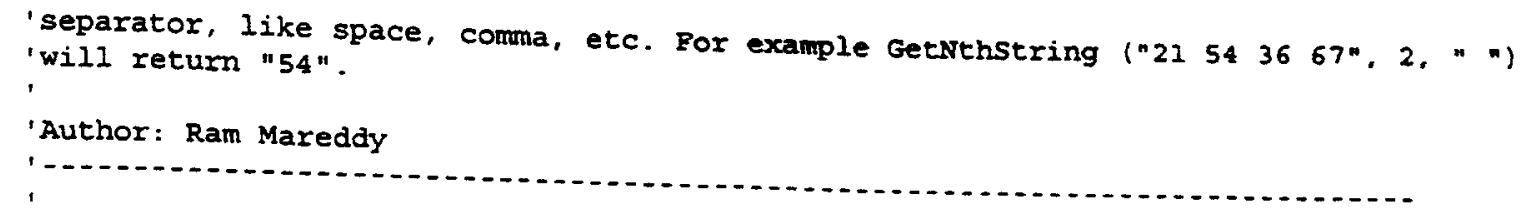




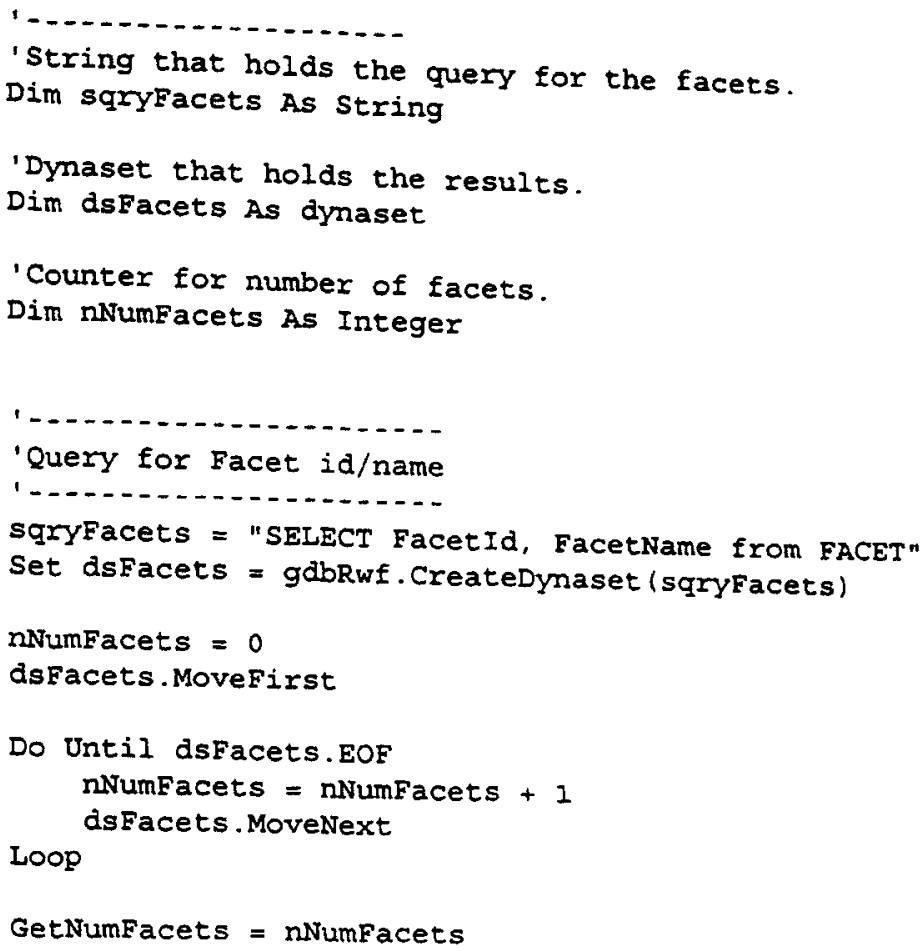

End Function

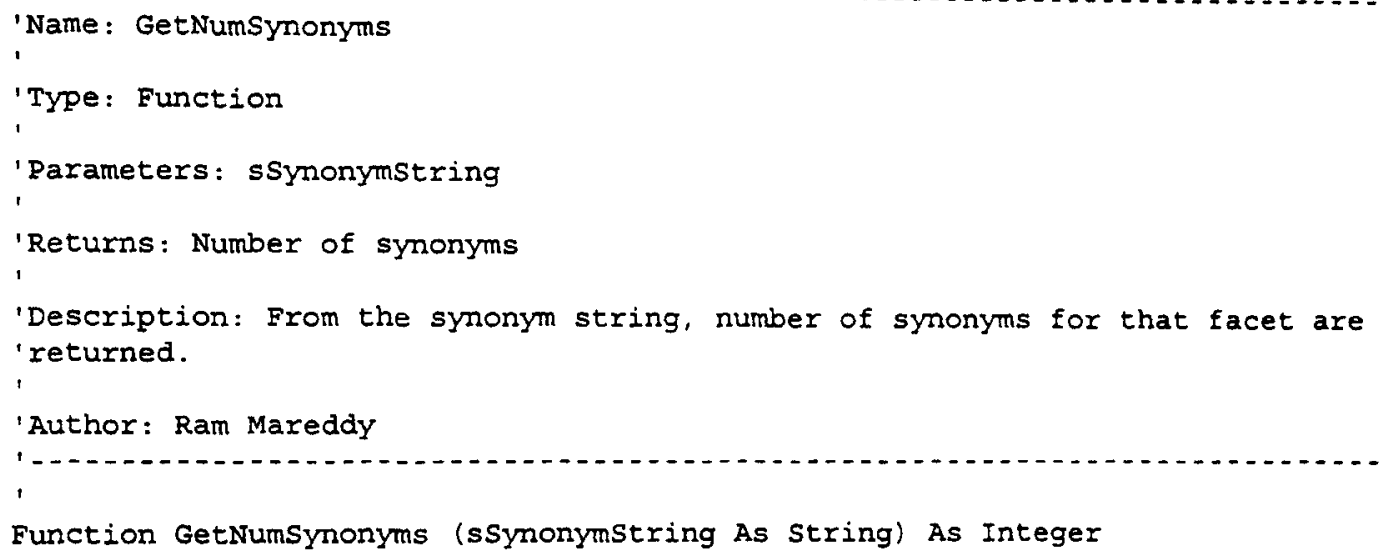


Else

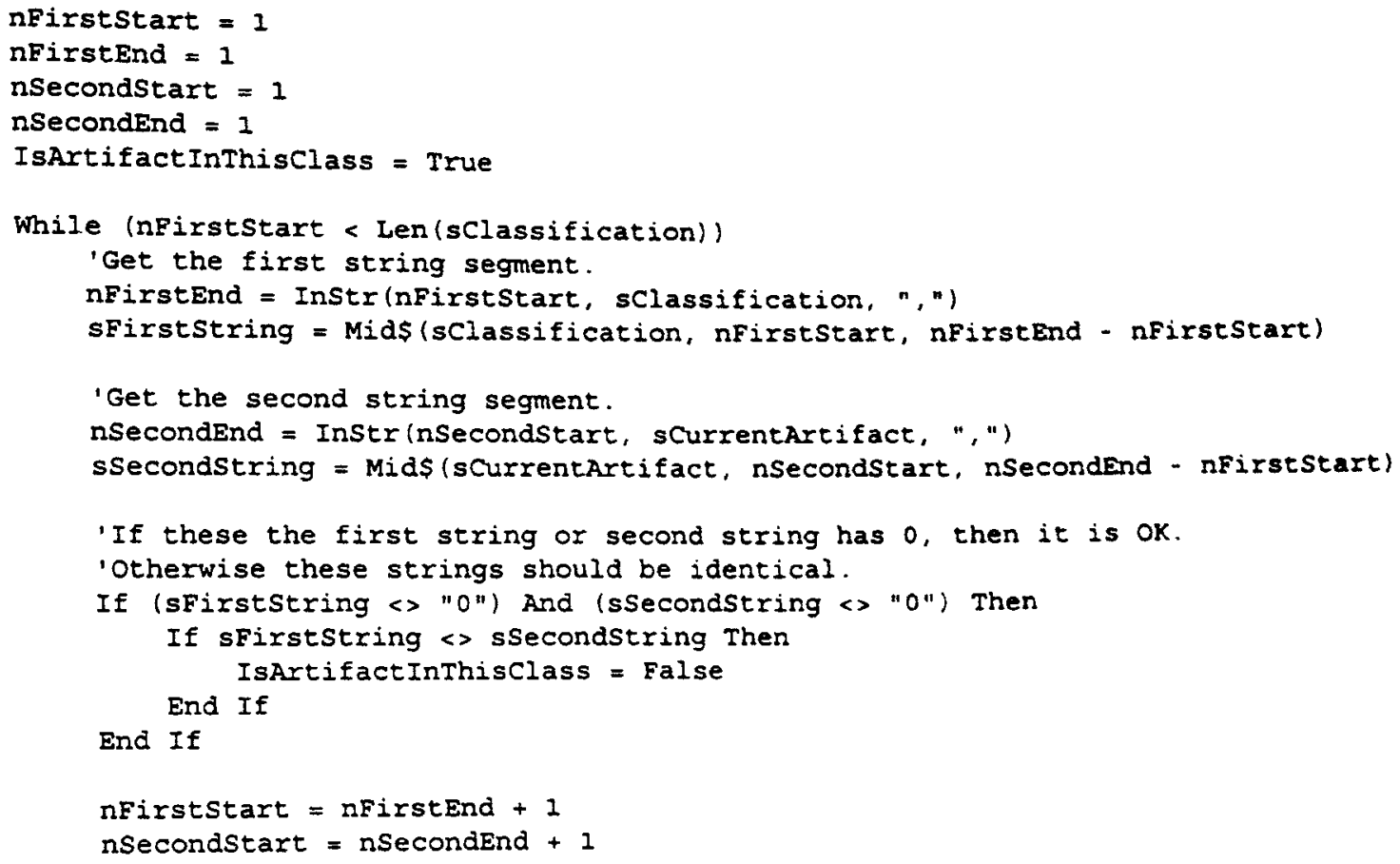

End If

End Function

'Name: IsSynonymocab

Type: Function

Parameters: sSymonym, sFacet

'Returns: True/False

'Description: Synonym might be in the thesaurus, but is it part of the 'vocabulary for that particular facet

'Author: Ram Mareddy

Function IsSynonymvocab (sSynonym, sFacet)

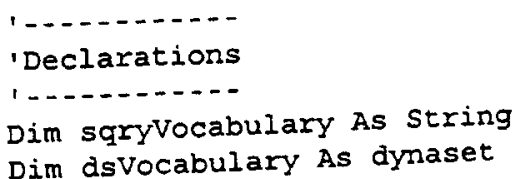


IsSynonymocab = dsvocabulary ("VocabularyId")

Else End If

IsSynonymivocab = gnNOT_IN_DATABASE

dsvocabulary. Close

End Function

Sub Login ()

frmLogin. Show Modal

End Sub

Name: Main

Type: Subroutine

'Parameters: None

'Returns: None

'Description: This is the start-up module. So, all the initialization and 'start-up code is placed here.

'Author: Ram Mareddy

'

Sub Main ()

'Read the global information.

GetPublicEnvironment gswindowsDirectory, gssystembirectory, gstempDirectory

'Read the initialization file.

GetAppEnvironment

- Open the database - if there are any problems, we will just quit.

openDb gudtRwfIni.sDatabase

'Login is for the purpose of metrics rather than security

Login

'Now show the main form.

Exmmain. Show

End sub

Name: OpenDb

'Type: Subroutine

'Parameters: psDatabase is the name of the database to open.

'Returns: None

Description. Try to open the database that is supplied as the parameter

any errors, inform the user and quit the application, because it's

If there are any errors, inform absolute must that the database be there

'Author: Ram Mareddy

$$
\text { ' }
$$


Sub Opendb (psDatabase As String)

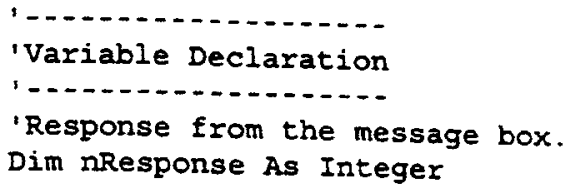

End Sub

'Name: SearchClassification

'Type: Function

'Parameters: sclassification is the classification string. 
Dim sCurrentartifact As string

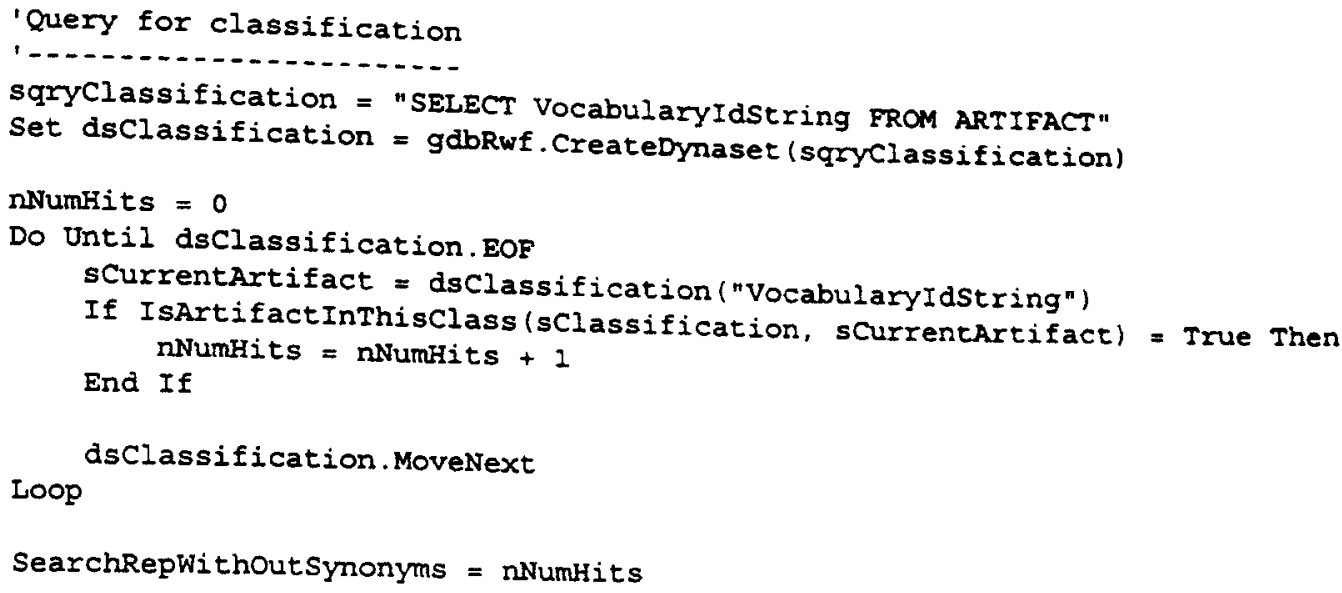




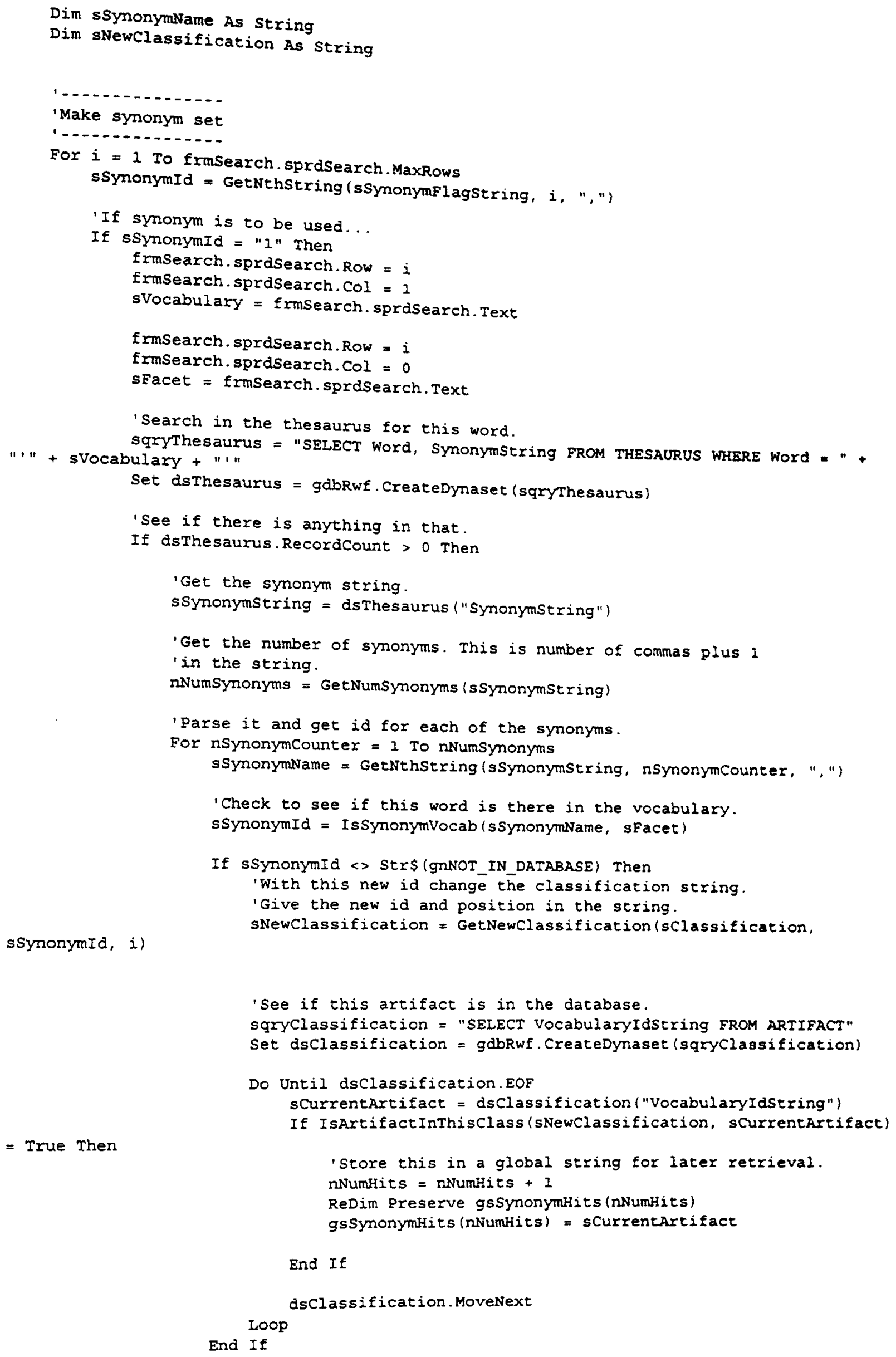




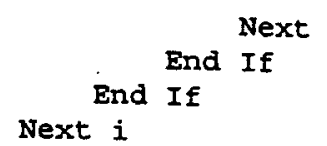

'dsclassification. Close

' dsthesaurus.Close

SearchRepwithsynonyms = nNumHits

End Function

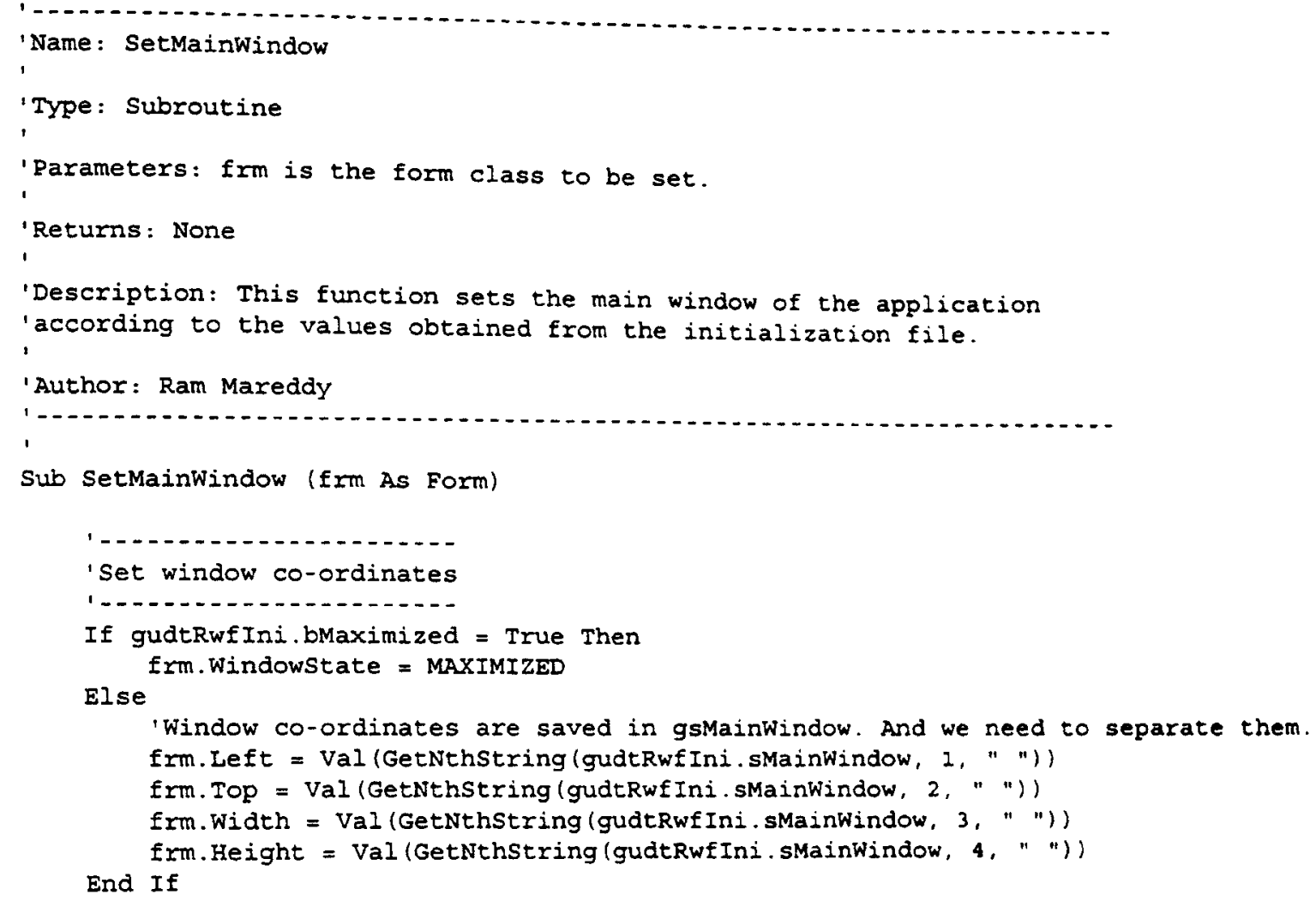

End Sub 


\section{VTA}

Ram R. Mareddy

Candidate for the Degree of

Master of Science

Thesis: AN IMPLEMENTATION OF THE FACETED CLASSIFICATION SYSTEM
FOR SOFTWARE REUSE

Major Field: Computer Science

Biographical:

Personal Data: Born in Eatoor, A.P., India, September 19, 1967, son of Vasantha and Somi Reddy.

Education: Received Bachelor of Technology degree in Mechanical Engineering from Jawaharlal Nehru Technological University in May 1988. Completed the requirements for the Master of Science degree at Oklahoma State University in December 1994.

Professional Experience: Management Trainee, Steel Authority of India Ltd., India May 1988 - April 1989; Engineer, Services and Trade Company, Sultanate of Oman, May 1989 - December 1989; Research Assistant, Department of Psychology, Oklahoma State University, August 1991 - November 1992; Programmer, A La Mode, Inc., Edmond, OK, May 1992 - August 1992; PC Programmer, Mirage Resorts, Inc., Las Vegas, NV, December 1992 - June 1994; Programmer/Analyst, Roadway Logistics, Akron, OH, August 1994 - Present. 